\title{
Molecular insights into the roles of RNA helicases during large ribosomal subunit assembly
}

\author{
Dissertation \\ for the award of the degree \\ “Doctor rerum naturalium” (Dr. rer. nat.)
}

of the Georg-August-Universität Göttingen

within the doctoral program IMPRS Molecular Biology

of the Georg-August University School of Science (GAUSS)

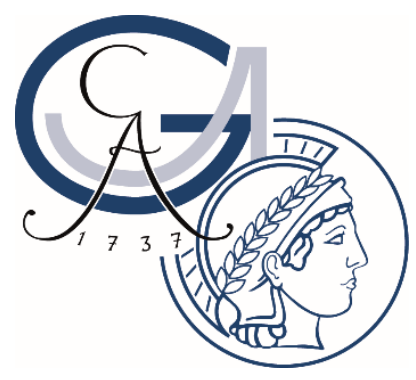

submitted by

Gerald Ryan Rabara Aquino

from Villasis, Pangasinan, Philippines

Göttingen, 2020 


\section{Members of the Thesis Committee}

Prof. Dr. Markus T. Bohnsack ( $1^{\text {st }}$ reviewer)

Department of Molecular Biology

University Medical Center Göttingen

Prof. Dr. Ralf G. Ficner (2 ${ }^{\text {nd }}$ reviewer)

Department of Molecular Structural Biology

Georg-August-University Göttingen

Prof. Dr. Henning Urlaub

Bioanalytical Mass Spectrometry Group

Max Planck Institute for Biophysical Chemistry, Göttingen

\section{Further members of the examination board}

Prof. Dr. Jörg Stülke

Institute for Microbiology and Genetics

Georg-August-University Göttingen

Prof. Dr. Michael Meinecke

Department of Cellular Biochemistry

University Medical Center Göttingen

Dr. Ricarda Richter-Dennerlein

Department of Cellular Biochemistry

University Medical Center Göttingen

Date of oral examination: 15.02.2021 


\section{Declaration}

Herewith I declare that I prepared the dissertation "Molecular insights into the roles of RNA helicases in large ribosomal subunit assembly" on my own and with no other sources and aids than quoted.

Göttingen, December 2020 


\section{Table of Contents}

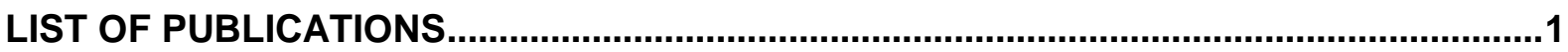

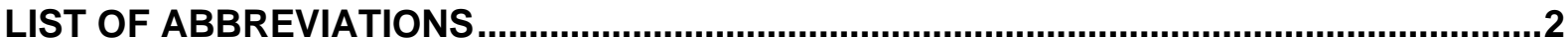

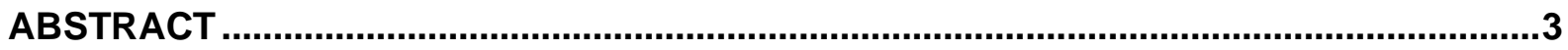

CHAPTER ONE: INTRODUCTION ..............................................................................

1.1 STRUCTURE AND FUNCTION OF RIBOSOME ..........................................................

1.2 GENERAL OVERVIEW OF RIBOSOME PRODUCTION IN YEAST .......................................

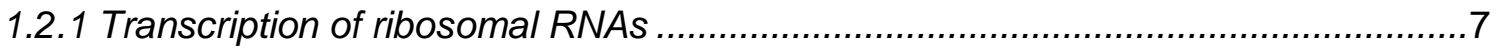

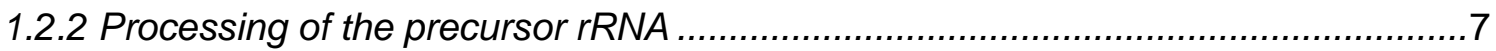

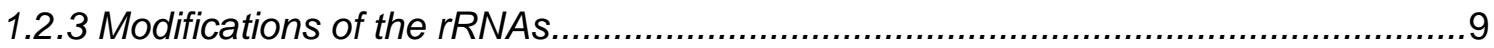

1.2.3.1 Structure and function of snoRNPs ......................................................... 10

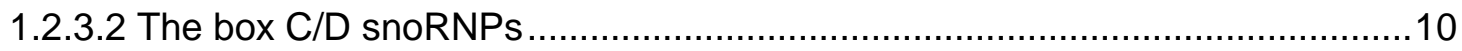

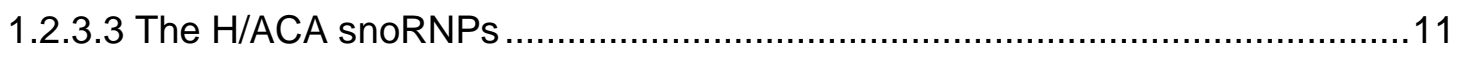

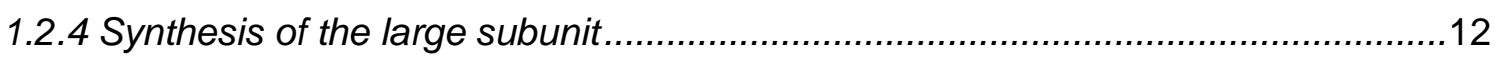

1.2.4.1 Key events and players during the early large subunit biogenesis .................12

1.2.4.2 Structural insights of nucleolar large subunit assembly ................................14

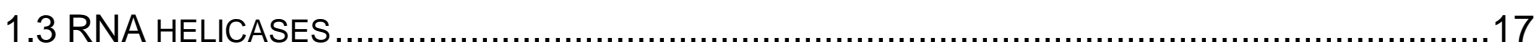

1.3.1. Classification and Structure of RNA helicases ................................................. 17

1.3.2 DExD/H proteins as RNA and RNP chaperones .............................................19

1.3.2.1 The translocating DEAH RNA helicases..................................................20

1.3.2.2 DExD box RNA helicases as local 'unwindase'...........................................21

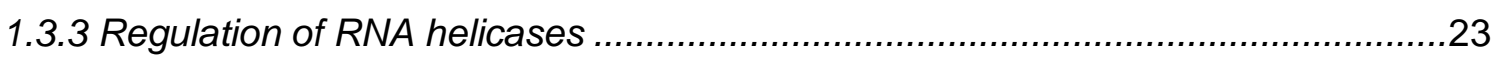

1.3.4 Roles of RNA helicases in different cellular pathways .....................................24

1.3.5 RNA helicase implicated in yeast ribosome biogenesis .....................................25

1.3.5.1 RNA helicases regulate snoRNA-pre-rRNA interactions ............................26

1.3.5.2 RNA helicases promote structural changes in pre-ribosomes ......................27

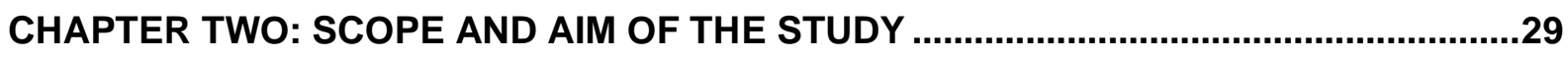

CHAPTER THREE: MANUSCRIPT I

The RNA helicase Dbp7 promotes domain V/VI compaction and stabilization of interdomain interactions during early 605 assembly

RNA helicase-mediated regulation of snoRNP dynamics on pre-ribosomes and rRNA 2'-O-methylation 
5.1 RNA HELICASES HAVE DISTINCT REMODELING FUNCTIONS TO DRIVE LSU MATURATION EVENTS. 134

5.2 DEXD-BOX RNA HELICASES DOMINATE RIBOSOME BIOGENESIS. 136

5.3 'ESSENTIALITY’ OF RNA HELICASES IMPLICATED IN RIBOSOME SYNTHESIS 137

5.4 TOWARDS UNDERSTANDING OF RNA HELICASE FUNCTIONS IN RIBOSOME BIOGENESIS ....138

5.4.1 Direct and indirect functions of RNA helicases. 138

5.4.2 Targeting of RNA helicases to their RNA substrates 140

5.4.3 Structural and catalytic function of RNA helicases. 141

5.4.4 Visualization of RNA helicases in pre-ribosomes and identification of RNA sequences contacted by the helicase active site. 143

5.5 FINE-TUNING OF RNA HELICASE FUNCTION 144

BIBLIOGRAPHY .146

ACKNOWLEDGEMENT .169 


\section{List of Publications}

Brüning,L., Hackert,P., Martin,R., Davila Gallesio,J., Aquino,G.R.R., Urlaub,H., Sloan,K.E. and Bohnsack,M.T. (2018) RNA helicases mediate structural transitions and compositional changes in pre-ribosomal complexes. Nat. Commun., 9, 5383. 


\section{List of Abbreviations}

\begin{tabular}{|c|c|}
\hline $\mathrm{AF}$ & assembly factor \\
\hline ATP & adenosine triphosphate \\
\hline CLASH & crosslinking and analysis of sequence hybrids \\
\hline CRAC & crosslinking and analysis of cDNA \\
\hline Cryo-EM & cryogenic electron microscopy \\
\hline Cryo-ET & cryo-electron tomography \\
\hline DEAH & aspartate-glutamate-alanine-histidine \\
\hline DExD & aspartate-glutamate-x-aspartate \\
\hline DMS & dimethylsulfide \\
\hline DNA & Deoxyribonucleic acid \\
\hline dsRNA & double-stranded RNA \\
\hline dsRNA & double-stranded RNA \\
\hline EJC & Exon junction complex \\
\hline ES & expansion segment \\
\hline ETS & external transcribe spacer \\
\hline GAC & GTPase activating center \\
\hline GKT & glycine-lysine-threonine \\
\hline ITS & internal transcribed spacer \\
\hline K-loop & kick-loop \\
\hline K-stem & kink stem \\
\hline KKX & lysine-lysine-x \\
\hline LSU & large subunit \\
\hline MIF4G & middle domain of elF4G \\
\hline mRNA & messenger RNA \\
\hline NPC & nuclear pore complex \\
\hline nt & nucleotide \\
\hline NTP & nucleotide triphosphate \\
\hline OB & oligosaccharide binding \\
\hline PET & polypeptide exit tunnel \\
\hline Pol I & polymerase I \\
\hline Pol III & polymerase II \\
\hline PTM & post-translational modification \\
\hline r-protein & ribosomal proteins \\
\hline rDNA & ribosomal DNA \\
\hline RLR & RIG-like receptors \\
\hline RMS & RiboMeth sequencing \\
\hline RNA & Ribonucleic acid \\
\hline RNA-IP & RNA immunoprecipitation \\
\hline RNP & ribonucleoprotein \\
\hline RRM & RNA recognition motif \\
\hline rRNA & ribosomal RNA \\
\hline SF & superfamily \\
\hline snoRNA & small nucleolar RNA \\
\hline snoRNP & small nucleolar RNP \\
\hline ssRNA & single-stranded RNA \\
\hline SSU & small subunit \\
\hline tRNA & transfer RNA \\
\hline UTR & untranslated region \\
\hline
\end{tabular}




\section{Abstract}

RNA helicases are enzymes present in all domains of life that are important in many aspects of RNA metabolism. They harbor a conserved helicase core that uses the energy of NTP binding and hydrolysis to change affinity to RNA, a biochemical property that provides RNA helicases mechanisms to unwind RNA duplexes. In the last years, there have been multiple lines of evidence that these specialized proteins can also displace proteins bound to RNAs and act as placeholders to promote intermediate RNA structures and folding. These RNA and ribonucleoprotein complex (RNP) remodeling functions underlie their involvement in many key cellular pathways and in the assembly of large RNPs, such as the ribosome. The synthesis of a ribosome is a very complex and dynamic processes consisting of the folding, processing and modification of the rRNA components coupled with the binding of ribosomal proteins ( $r$ proteins). These events are highly orchestrated and involve numerous assembly factors (AFs), such nucleases, RNA helicases and RNA-binding proteins. Many RNA helicases belonging to DEAH and DExD subfamily act as molecular machines to drive structural rearrangements essential in the ribosome assembly process. However, the precise molecular functions of many RNA helicases implicated in this pathway and the underlying mechanisms of how they promote maturation events remain poorly understood. In this work, biochemical and molecular methods combined with transcriptome- and proteome-wide approaches were employed to gain insights into the molecular functions of the two DExD box RNA helicases Dbp3 and Dbp7 in yeast ribosome biogenesis. In vitro NADH-coupled ATP assays show that both RNA helicases are RNA-dependent ATPases. Pre-rRNA processing analyses implicate both in the large subunit (LSU) biogenesis. MS-based analysis of protein composition of purified Dbp7-containing particles identifies early-binding AFs and r-proteins, indicating a potential role of Dbp7 in the initial stages of LSU production. Moreover, compositional analyses of pre-60S from cells expressing and lacking Dbp7 suggests a role of Dbp7 in facilitating the recruitment of AFs and r-proteins. In vivo PAR-CRAC reveals crosslinking sites of Dbp7 on the $25 \mathrm{~S}$ rRNA consistent with the known binding sites of these proteins. All these findings support a remodeling function of $\mathrm{Dbp} 7$ that leads to the compaction and stabilization of early pre-60S particles. Meanwhile, in the absence of Dbp3, many sites in the 25S rRNA were observed to have sub-stoichiometric 2'-O-methylation. Northern blot and qPCR analyses of snoRNAs guiding these affected sites reveal accumulation of several snoRNAs in the pre-ribosomal complexes when Dbp3 is lacking. Furthermore, overexpression of a snoRNA guiding several, non-proximal modifications recues the observed inefficient modification. Collectively, these data imply a role of Dbp3 in regulating snoRNP dynamics and pre-rRNA 2'-O-methylation during LSU biogenesis. Overall, this work provides important insights into the functions of Dbp3 and Dbp7 and therefore contributes to the functional characterization of RNA helicases involved in the early stages of LSU biogenesis. 


\section{Chapter One: Introduction}

\subsection{Structure and function of ribosome}

Ribosomes are macromolecular machines that decode the genetic information carried by messenger RNA (mRNA) to produce the proteome of all cells in nature. These highly conserved ribonucleoprotein (RNP) complexes are composed of two subunits: the large subunit (LSU/60S) and small subunit (SSU/40S). The LSU is twice the size of the SSU and harbors the peptidyltransferase center (PTC), which catalyzes peptide bond formation. In contrast, the SSU serves as the decoding center bringing the mRNA and the aminoacylated transfer RNA (tRNA) together to read the genetic code.

The eukaryotic cytoplasmic $80 \mathrm{~S}$ ribosome is composed of 4 ribosomal RNAs (rRNAs) and approximately 80 ribosomal proteins ( $r$-proteins). The LSU contains three rRNAs, namely $28 \mathrm{~S}$ (human; 5025 nucleotides (nt))/25S (yeast Saccaharomyces cerevisiae, hereafter referred as yeast; $3396 \mathrm{nt}$ ), $5.8 \mathrm{~S}$ (158 nt), 5S (121 nt), and 47 (humans)/46 (yeast) r-proteins (Ben-Shem et al., 2011; Khatter et al., 2015). In comparison, the SSU is comprised of the 18S rRNA (human 1808/yeast $1800 \mathrm{nt}$ ) together with 33 r-proteins. Although highly conserved, eukaryotic ribosomes are evolved from their prokaryotic counterparts with the acquisition of eukaryoticspecific r-proteins, r-protein extension, rRNA expansion segments (ES) and many additional rRNA modifications (Ben-Shem et al., 2011; Gerbi, 1986; Klinge et al., 2012; Petrov et al., 2014b; Taoka et al., 2016; Wilson \& Doudna Cate, 2012).

The yeast ribosome is the most studied and characterized eukaryotic ribosome. A recently available X-ray crystallography structure of yeast $80 \mathrm{~S}$ ribosome revealed a universally conserved core surrounded by eukaryotic-specific features (Ben-Shem et al., 2011, Petrov et al., 2014b). Nevertheless, the rRNA maintains the highly conserved secondary structural domains. The $18 \mathrm{~S}$ rRNA folds into four structural domains, the 5', central, 3' major and 3' minor, which upon assembly with the respective r-proteins give rise to the body, shoulder, platform, head and beak structures seen in three-dimensional space (Figure 1A; Figure 1B). Meanwhile, the $5.8 \mathrm{~S}$ and the $25 \mathrm{~S}$ of LSU fold together to form six conserved domains (I-VI) of secondary structures (Petrov et al., 2014a). These domains further fold into tertiary structures upon assembly with r-proteins and form the characteristic structural modules of a mature LSU, such as the central protuberance, the L1 stalk, and the acidic stalk (Figure 1C; Figure 1D). In all domains of life, the functional core comprising the PTC and the polypeptide exit tunnel (PET), and the GTPase activating center (GAC) in LSUs as well as the tRNA binding sites, the decoding center, and the mRNA entry and exit sites in SSU, remain highly conserved (Melnikov et al., 2012) 
A

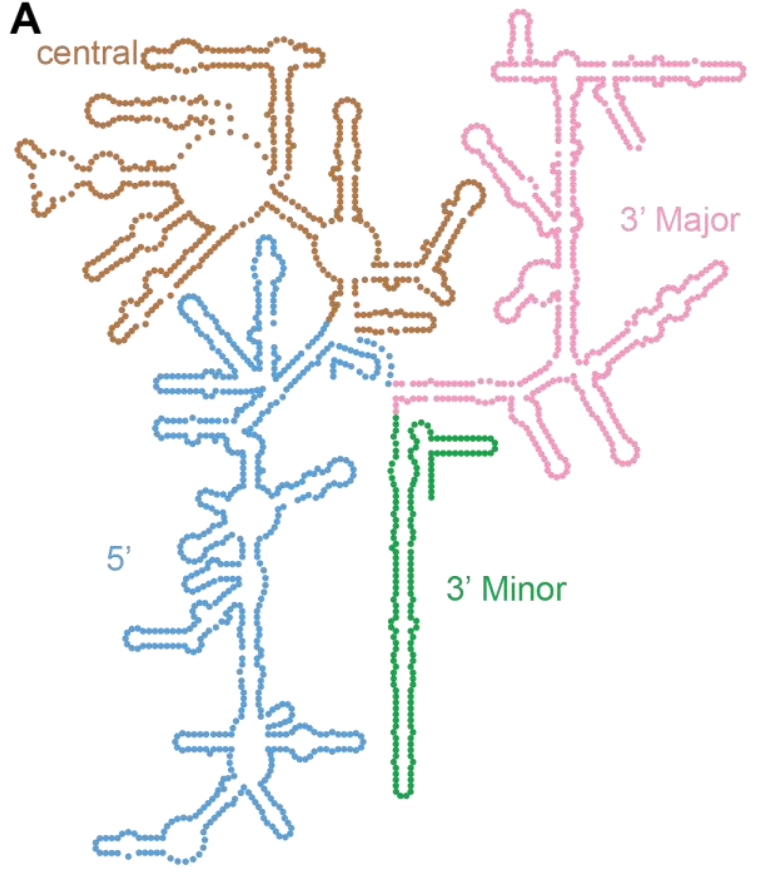

C

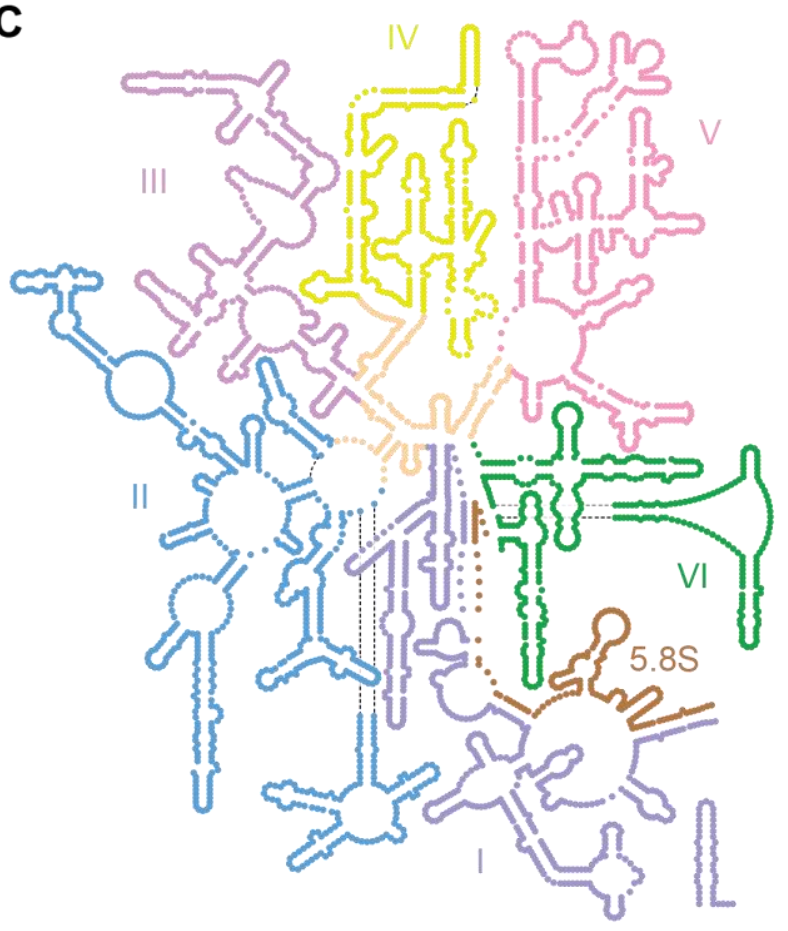

D

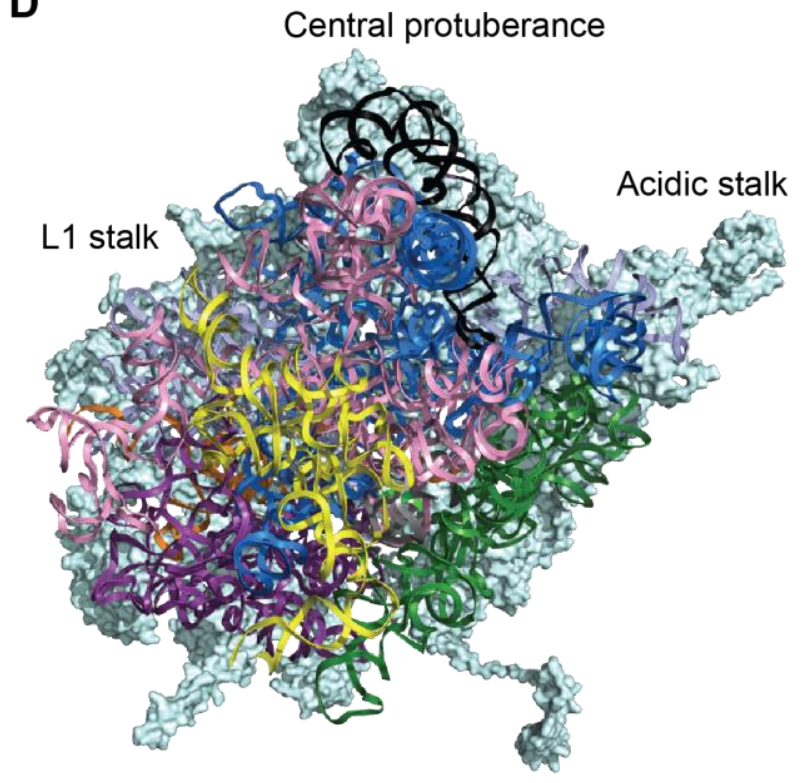

B

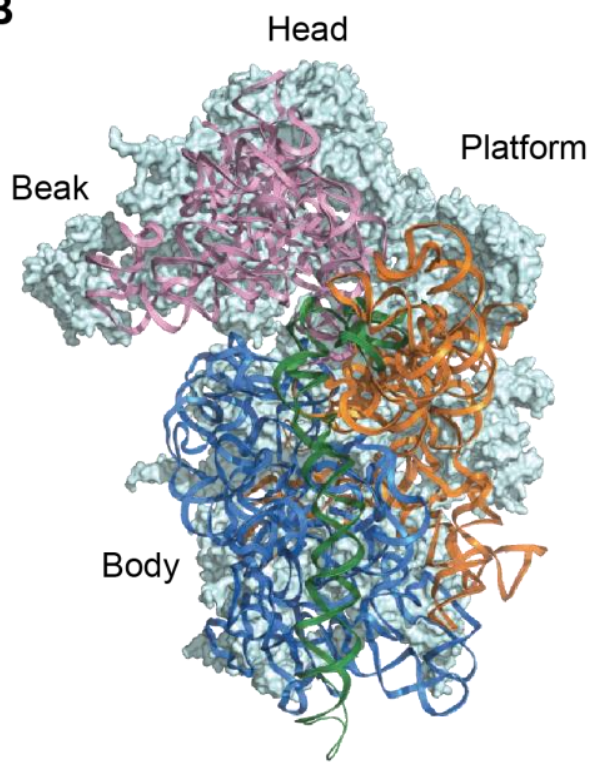

Figure 1. Secondary structure of S.cerevisiae rRNAs and their three-dimensional arrangement in mature ribosomal subunits. (A) $18 \mathrm{~S}$ rRNA secondary structure with the different structural domains consisting of the 5', central, 3' major, and 3' minor shown in different colors. Secondary structure maps were adapted from Petrov et al. (2014a). (B) Folding of the 18S rRNA leading to the formation of beak, head, body and platform structures in mature small subunit seen from the subunit interface side. Structural domains are highlighted with the same color as in $A$. Tertiary structures were reconstructed from available crystal structure of mature ribosomal subunits (Ben-Shem et. al., 2011). rRNAs were overlaid and the r-proteins are shown in cyan. (C) Secondary structure of 25S, 5.8S, and 5S rRNAs. 25S rRNA folds into six structural domains (I-VI) highlighted in different colors respectively. 5.8S rRNA forms secondary structure with domain I of 25S rRNA (D) Folding of the LSU rRNAs in mature subunit viewed from the subunit interface. rRNAs and the different domains are shown in similar scheme as in C. Characteristic structural modules including the L1 stalk, central protuberance and acidic stalk are indicated. 


\subsection{General overview of ribosome production in yeast}

The production of ribosomes is one of the most energy-consuming processes in a cell and has a rate of 2000 ribosomes per minute in an actively dividing yeast cell (Warner, 1999). Ribosome production is tightly linked to nutrient availability, cell cycle progression and stress conditions (de la Cruz et al., 2018). Defined stages of the biogenesis pathway occur in three different cellular compartments. The process starts in nucleolus, then it continues in the nucleus and final subunit maturation steps occur in the cytoplasm. It involves a series of events including the transcription of ribosomal DNA ( $\mathrm{rDNA}$ ), processing, modification and folding of precursor ribosomal RNAs (pre-rRNA), correct assembly of r-proteins and the transport of ribosomal precursor particles from the nucleus to the cytoplasm. These processes are coupled with structural transitions that are highly orchestrated, and with the transient association and dissociation of numerous small nucleolar ribonucleoproteins (snoRNPs) and approximately 200 assembly factors (AF) including nucleases, nucleotide triphosphate (NTP)-dependent RNA helicases, AAA-ATPases, GTPases, kinases and phosphatases, and RNA-binding proteins (reviewed in Woolford \& Baserga, 2013). Many of these AF have structural functions to promote stability of pre-ribosomal particles and network of molecular interactions, while others have enzymatic functions that uses energy to drive irreversible remodeling of these molecular interactions (reviewed in Kressler et al., 2010; Strunk \& Karbstein, 2009).

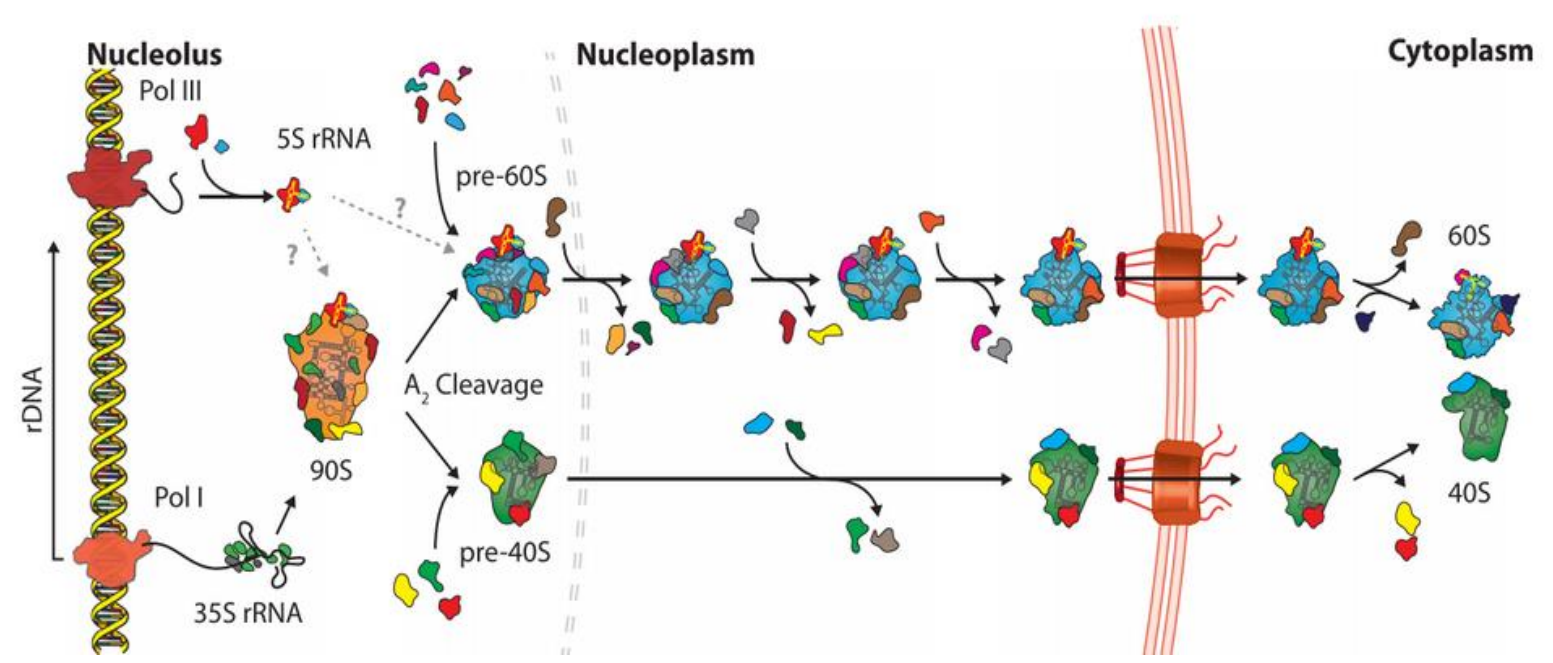

Figure 2. Ribosome biogenesis in S. cerevisiae. Synthesis starts with nucleolar transcription of rDNA generating a single polycistronic transcript containing the 18S, $5.8 \mathrm{~S}$ and $25 \mathrm{~S}$ rRNAs. Cotranscriptional assembly with snoRNAs, AFs and r-proteins generates $90 \mathrm{~S}$ pre-ribosomal particles that split into pre-40S and pre-60S subunits upon cleavage at $\mathrm{A}_{2}$. Both undergo independent maturation as they travel from the nucleolus across the nucleoplasm to the cytoplasm, where final maturation occurs before the two sub-units assemble. The mature 5s rRNA is delivered into the nucleolus as a pre-assembled RNP. Image taken form Gerhardy et al. (2014). 
Ribosome biogenesis begins with the nucleolar synthesis of the $35 \mathrm{~S}$ primary transcript by RNA polymerase I (Pol I) (Figure 2). As the transcript emerges from the polymerase, it associates with many r-proteins and numerous AFs to generate the earliest pre-ribosomal particle called the SSU processeome (Barandun et al., 2017; Gerhardy et al., 2014; Kornprobst et al., 2016). Concomitant to synthesis, the pre-rRNA undergoes covalent modifications carried out mostly by snoRNPs (Sloan et al., 2017; Watkins \& Bohnsack, 2012), as well as cleavage events to generate the mature rRNAs (Henras et al., 2015; Kos \& Tollervey, 2010). One key cleavage event at site A2 leads to the production of the earliest small and large pre-ribosomal particles, each then undergoes independent maturation pathway (Udem \& Warner, 1972). Both undergo a series of remodeling steps, with a greater extent of remodeling of pre-LSU particles as they traverse the nucleus. Such remodeling events involve numerous protein exchanges, and folding and rearrangement of the rRNA. During this cytoplasmic assembly stage, the different domains are being arranged and pre-ribosomal particles achieve a conformation nearly close to the mature small and large sub-unit (Klinge \& Woolford, 2019). Upon export into the cytoplasm, final rearrangement and proofreading steps take place to assess the functionality of both subunits for translation.

\subsubsection{Transcription of ribosomal RNAs}

In yeast cells, most of the rRNAs are synthesized by Pol I from tandemly repeated rDNA units (150-200) located in chromosome XII (Long \& Dawid, 1980). A primary 35S transcript is generated and harbors the rRNAs integral for both LSU (25S and 5.8S) and SSU (18S) (Figure 3). Aside from the mature rRNA sequences, the $35 \mathrm{~S}$ transcript also contains sequences that are excised during maturation. These include two internal transcribed spacers (ITS), ITS1 and ITS2, which separates the mature rRNA sequences, as well as external transcribed spacers (5' ETS and 3' ETS) flanking the 5' and 3' end of the 5.8S and 25S, respectively. The third rRNA component of LSU, the 5S rRNA, is synthesized in a reverse direction by the RNA polymerase III (Pol III). rDNA transcription occurs in the nucleolus and several transcription events occur in an active rDNA unit as evidenced by chromatin (Miller) spreads under an electron microscope (Miller \& Beatty, 1969).

\subsubsection{Processing of the precursor rRNA}

The polycistronic 35S pre-rRNA transcript undergoes a series of endonucleolytic and exocnucleolytic cleavage events (Figure 3 ) to remove the four transcribed spacers and generate the mature rRNAs (Henras et al., 2015; Mullineux \& Lafontaine, 2012; Venema \& Tollervey, 1995). Different approaches including microscopy and biochemical assays have revealed that majority of pre-rRNA processing (70\%) occurs co-transcriptionally in yeast (Kos \& Tollervey, 2010; Osheim et al., 2004). However, this is not a necessity as yeast cells can still 
synthesis both ribosomal subunits from pre-rRNAs processed post-transcriptionally (Trapman et al., 1975; Udem \& Warner, 1972).

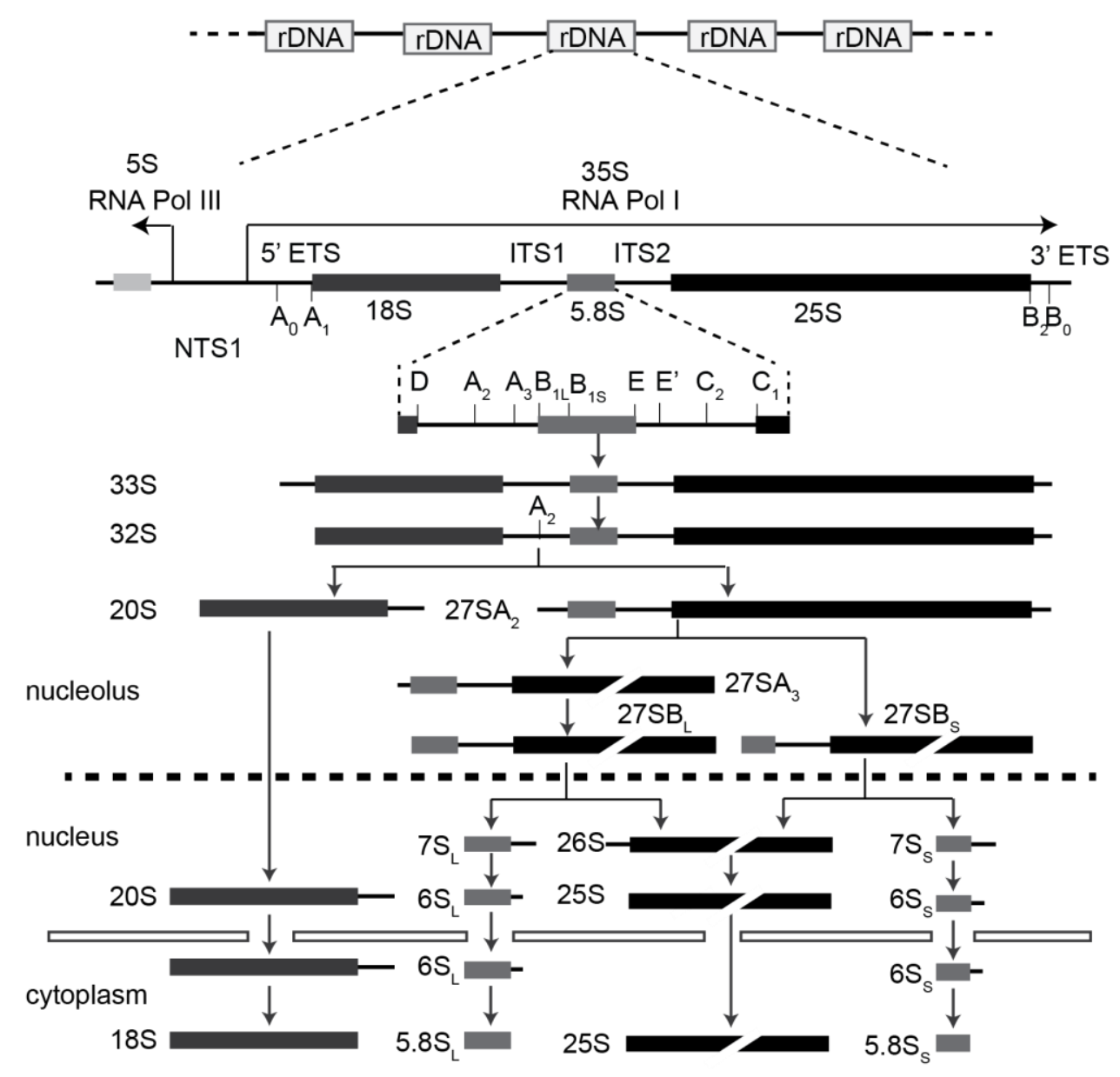

Figure 3. Schematic representation of S. cerevisiae rDNA locus, rDNA transcription and prerRNA processing pathway. A single unit of 150-200 rDNA repeats is represented. Independent transcription by RNA pol I and RNA pol III in opposite directions (indicated by the arrows) generate the $35 \mathrm{~S}$ and $5 \mathrm{~S}$ (light grey) pre-rRNAs respectively. The 35S pre-rRNA contains the mature sequence of 5.8S (grey), 18S (black; short), and 25S (black; long) rRNAs interspersed by two internal transcribed spacers (ITS1 and ITS2) and flanked by two external transcribed spacers (ETS1 and ETS2). ETS and ITS are removed in a series of endo- and exonucleolytic cleavages indicated by letters across the three different cellular compartments. Diagram adapted from Baserga and Woolford (2013).

Pre-rRNA processing commences with the removal of the 5' ETS in a two-step endonucleolytic cleavage at sites $A 0$ and subsequently at site $A 1$ by the endonuclease Utp24 (Wells et al., 2016). This results to the production of $33 S$ and $32 S$ pre-rRNAs, respectively (Beltrame et al., 1994; Hughes \& Ares, 1991). Cleavage within ITS1 (A2 site) separates the pre-rRNA of the LSU (27SA) and SSU (20S) leading to the production of the earliest pre-ribosomal particles, $66 S$ and $43 S$ particle, respectively, and their subsequent independent maturation (Udem \& Warner, 1972). The pre-SSU complex immediately exits the nucleolus and is exported to the cytoplasm where the $20 \mathrm{~S}$ pre-rRNA is then finally processed to $18 \mathrm{~S}$ through cleavage at site 
D by the endonuclease Nob1 (Fatica et al., 2003a; Pertschy et al., 2009). In the case of the large subunit pre-rRNA, it undergoes more complex processing concomitant with extensive remodeling of the pre-60S particle as it is transported to the nucleoplasm (Klinge \& Woolford, 2019). The 27SA is processed in the nucleolus via two alternative pathways. In most cases, i.e. $85-90 \%$, cleavage at A3 site within ITS1 by the MRP RNase produces the $27 \mathrm{SA}_{3}$ intermediates (Chu et al., 1994; Lindahl et al., 2009; Lygerou et al., 1996; Schmitt \& Clayton, 1993; Shuai \& Warner, 1991). Subsequently, the remaining ITS1 sequences until the B1s site is removed by the exonucleases Rat1 and Rrp17 to generate the 27SB pre-rRNA (Henry et al., 1994; Oeffinger et al., 2009). Alternatively, some of the 27SA (15\%) are directly cleaved at the $B 1 \mathrm{~L}$ site generating the $27 \mathrm{SB}_{\llcorner}$pre-rRNA containing a $5.8 \mathrm{~S}$ with a different 5 ' end referred as $5.8 S_{\mathrm{L}}$. In any case, both $27 \mathrm{SB}$ species undergo identical processing at the site $\mathrm{C} 2$ within ITS2, separating the two pre-rRNAs $25.5 S$ and $7 S_{S}$ or $7 S_{L}$. The remaining 5 ' end of the $25.5 S$ pre-rRNA is subsequently removed by Rat1 and Xrn1 to generate the mature 25S rRNA (Geerlings et al., 2000). Alongside, the 7S $/ 7 S_{\llcorner}$pre-rRNA are processed at the 3' end until site E by the Rrp6-Rrp47-Mpp6-Mtr4-associate exosome to produce the mature 5.8SL or 5.8SS (Henry et al., 1994; Kilchert et al., 2016; Mitchell et al., 1996; Schuller et al., 2018). Despite this highly conserved 6 nt-difference between the two 5.8S rRNA species, both have been observed in translating ribosomes and have been speculated to promote translation of specific subset of mRNAs (Schmitt \& Clayton, 1993).

\subsubsection{Modifications of the rRNAs}

In addition to processing, the pre-rRNAs are also extensively modified. These modifications can be installed co-transcriptionally or post-transcriptionally (Kos \& Tollervey, 2010). To date, modifications in 113 sites have been reported and these include 2'-O-methylations, pseudouridylations, methylations and acetylations (reviewed in Sloan et al., 2017). Most of these modifications are non-essential as loss of few individual modifications still allows yeast cells to remain viable and ribosome biogenesis to proceed; however, few modifications are crucial for cells for exhibit normal growth, e.g. $\mathrm{m}^{1} \mathrm{acp}^{3} \Psi 1191$ in 18S rRNA (Liang et al., 2009; Sharma \& Lafontaine, 2015; Sloan et al., 2017). Notably, Most of these modifications cluster in the regions important for catalytic activity of the ribosome (Brimacombe et al., 1993; Decatur \& Fournier, 2002). It has been shown that these modifications provide stability of the secondary and tertiary structures of the rRNA, which in turn contributes to the functional integrity and efficiency of ribosomes (Baudin-Baillieu et al., 2009; Baxter-Roshek et al., 2007; Decatur \& Fournier, 2002; Gigova et al., 2014; Jack et al., 2011; King et al., 2003; Polikanov et al., 2015). With the development of quantitative high-throughput approaches, some sites were found to be partially modified (Birkedal et al., 2015; Buchhaupt et al., 2014; Krogh et al., 2017; Taoka 
et al., 2016). These observations suggest that rRNA modifications contribute to ribosome heterogeneity and further supports the growing body of evidence of specialized ribosomes.

\subsubsection{Structure and function of snoRNPs}

Majority of the rRNA modifications are 2'-O-methylations and pseudouridylations, which are mostly installed by snoRNPs (Sloan et al., 2017). These snoRNPs are composed of a small nucleolar RNA (snoRNA) and several proteins, including one that has the enzymatic function to carry out the nucleotide modification (Watkins \& Bohnsack, 2012). The snoRNA component guides modification to the target site by baseparing with the pre-rRNA sequences. They can be classified either as box C/D or box H/ACA based on the modification being guided and conserved sequence motifs (Balakin et al., 1996; Kiss-László et al., 1998; Ni et al., 1997). These two types have also distinct RNA structures and set of associated proteins (Reichow et al., 2007).

Approximately 75 small nucleolar RNPs (snoRNPs) are involved in yeast ribosome biogenesis. Some of these snoRNPs can install two or more modifications at different sites but many only catalyze a single modification. Aside from modifications, snoRNP interaction with pre-rRNA has been suggested to chaperone RNA folding thereby facilitating pre-rRNA processing (Bachellerie et al., 2002; Henras et al., 2017; Kiss et al., 2010; Ojha et al., 2020; Sloan et al., 2017; Watkins \& Bohnsack, 2012). Meanwhile, two yeast snoRNAs, snr30 and snR190, have no cognate rRNA modification identified to date (Birkedal et al., 2015; Kiss-László et al., 1996; Krogh \& Nielsen, 2019; Kudla et al., 2011; Sloan et al., 2017). It has been recently reported that snR190 has another putative base-pairing site on the 25S rRNA and therefore postulated to have a rRNA folding chaperone function (Joret et al., 2018)

\subsubsection{The box C/D snoRNPs}

The box C/D snoRNPs catalyze the covalent attachment of a methyl group at the 2'-OH position of the ribose and acetylation of two sites guided by snR4 and snR45 (Sharma et al., 2017). Such 2'-O-ribose methylation occurs in 55 sites in yeast rRNA and contributes to the folding properties, hydrophobicity and stability against hydrolysis of the rRNA (Helm, 2006; Kiss-László et al., 1996; Ojha et al., 2020; Sloan et al., 2017; Watkins \& Bohnsack, 2012).

The box C/D snoRNA component is usually characterized by the presence of box C/D motif at the 3'- and 5'-end and an internal box C'/D' motif located between the two guide sequences (Kiss-László et al., 1998) (Figure 4A). Basepairing of the C/D motifs forms a secondary structural motif called the Kink-stem (K-stem), while the C'/D' motifs forms a Kick-loop (K-loop) (Reichow et al., 2007; Watkins et al., 2000). Structural insights from archeal box C/D snoRNP 
suggests that each of these structural motifs provides a binding center for the core protein components Nop1 (Fibrillarin in humans), Nop58, Nop56, and Snu13 (15.5K/NHPX in humans) (Bleichert et al., 2009; Lin et al., 2011). Nop58 and Nop56 preferentially bind to C/D and C'/D' motifs respectively, while Snu13 molecules bind to both and contacts the sheared GA basepairs (Qu et al., 2011; Szewczak et al., 2002; van Nues \& Watkins, 2017; Watkins \& Bohnsack, 2012) (Figure 4A). The catalytic activity is endowed by the methyltransferase Nop1 (or Kre33) which binds to both C/D and C'/D' motifs and partially to the guide-substrate duplex (Cahill et al., 2002; Sharma et al., 2017; Tollervey et al., 1993). The guide sequences, each consisting of 10-21 nt that basepair with the target pre-rRNA sequence, specify modification of, typically, the $5^{\text {th }}$ nt upstream the box D or D' sequence (Kiss-László et al., 1996; van Nues et al., 2011).
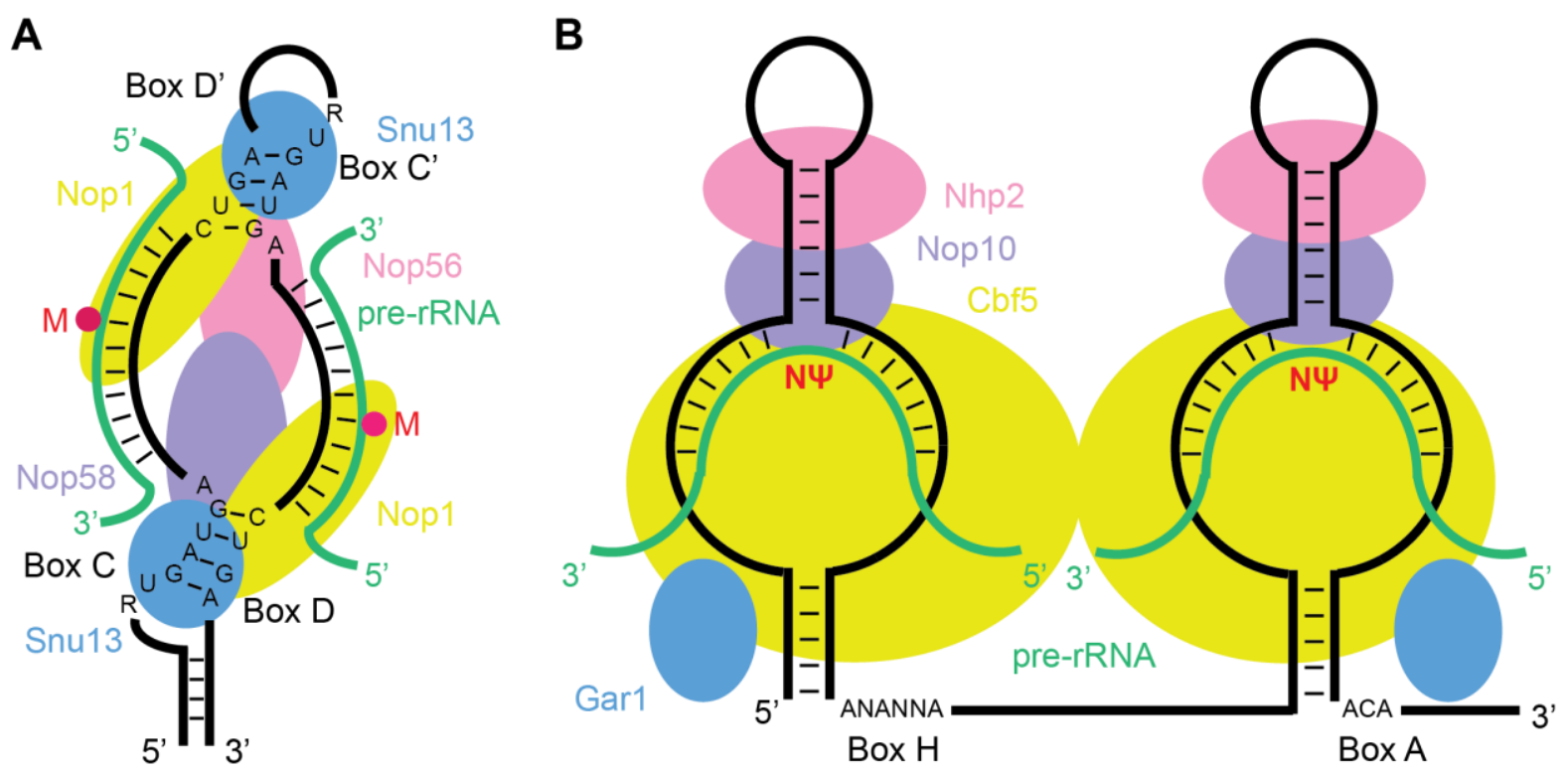

Figure 4. Schematic representation of yeast snoRNP complex formation and pre-rRNA targeting. (A) Predicted binding positions of box C/D snoRNP core proteins Nop1 (yellow), Nop56 (pink), Nop58 (purple), and Snu13 (blue) on a snoRNA (black). Methylation (magenta) of the prerRNA (green) is installed at the $5^{\text {th }}$ nt upstream of the D or D' sequence. (B) Predicted binding of H/ACA snoRNPs proteins consisting of Gar1 (blue), Nop10 (purple), Cbf5 (yellow), and the guide snoRNA (black) with the target pre-rRNA (green) to catalyze pseudouridylation of a uridine (magenta). Diagrams adapted from Watkins and Bohnsack (2012) and van Nues and Watkins (2017).

\subsubsection{The H/ACA snoRNPs}

Pseudouridylation of $r$ RNA is carried out by box H/ACA snoRNPs. This isomerization of uridine occurs at 45 positions in yeast rRNA and increases the capacity of hydrogen bonding, stability of base staking, and the rigidity of sugar-phase backbone (Davis, 1995; Ojha et al., 2020; Sloan et al., 2017).

$\mathrm{H} / \mathrm{ACA}$ snoRNAs are characterized by the presence of two stem loops and two conserved sequence motifs, the $\mathrm{H}$ box and ACA motifs (Figure 4B). The two stem loops are separated 
by a single stranded hinge region containing the $\mathrm{H}$ box sequence motif. The ACA motif, meanwhile, is located after the second stem loop and is three nucleotides away from the 3' end of the snoRNA. A pseudouridylation pocket is generated within the loop region of each hairpin structures and contains a guide sequence of 4-8 nt that basepair to the RNA target (Kiss et al., 2010; Watkins \& Bohnsack, 2012). In most cases, one H/ACA snoRNA guides one pseudouridylation site but few can target multiple sites, e.g. snR49, snR43 and snR191 (Sloan et al., 2017). The protein components include Cbf5 (Dyskerin in humans), Gar1, Nop10, and Nhp2. Cbf5 is the catalytic pseudouridine synthetase and is also key in maintaining a linear conformation of the two hairpin structures (Lafontaine et al., 1998; Li \& Ye, 2006). To date, there is no available structure of a yeast box H/ACA snoRNP.

\subsubsection{Synthesis of the large subunit}

The yeast LSU is composed of the 25S rRNA, 5.8S rRNA, 5S rRNA and 46 r-proteins. This complex composition compared to SSU warrants for an intricate assembly process. The assembly pathway can be defined into six stages based on the processing of pre-rRNAs within the pre-60S particle (reviewed in Konikkat \& Woolford, 2017). Unlike the SSU, only the 'very early' stage of LSU assembly occurs co-transcriptionally. This is the period when the pre-rRNA is compacted to SSU processeome upon association of r-proteins and early acting AFs until cleavage at $A_{2}$ site to produce the earliest pre-60S particle. The 'early' stage then commences with the removal of the ITS1 spacer sequences from 27SA to generate 27SB in 66S preribosome. This is followed by the cleavage at C2 site in ITS2 of 27SB to generate 7S and $27 \mathrm{~S}$ pre-rRNA and the exit of pre-66S from the nucleolus, both of which comprise the 'middle' stage of assembly. 'Late' stage assembly involves the removal of ITS2 and remodeling of the central protuberance. In the next stage, the nascent pre-60S is transported from the nucleus to the cytoplasm through the nuclear pore complex (NPC) (Gerhardy et al., 2014; Sloan et al., 2016). In the cytoplasm, the final stage of assembly includes the last pre-rRNA processing steps to generate the mature 5.8S, incorporation of the last few $r$-proteins, release of remaining AFs and surveillance steps to check for translation-competence.

\subsubsection{Key events and players during the early large subunit biogenesis}

The formation of the very early pre-60S commences co-transcriptionally and after A2 cleavage. It involves assembly of numerous r-proteins and approximately one-third of AFs with the nascent pre-rRNA to initiate folding of the pre-rRNA into more compact and stable state, along with chemical modifications of pre-rRNA mostly by snoRNPs and processing of the 3' ETS and ITS1. As transcription is finished, the first recognizable pre-ribosomal particle destined to be LSU is formed. The early pre-rRNA folding and processing events can be a highly dynamic, heterogeneous and very transient process. Due to these factors coupled with technical 
limitations to date, insights into the assembly and maturation of the initial pre-60S remains limited.

\subsection{Folding of rRNA secondary structure domains}

The 25S rRNA consists of six conserved domains of secondary structure (domains I-VI), which further fold to form tertiary structures (Figure 1C-D). Domains I and II, together with the 5.8S rRNA, constitute the solvent-exposed side of the large subunit. Meanwhile, domains IV and V, make up the subunit interface where the functional centers are located. Domains III and IV link one of the sides of the solvent-exposed surface to the subunit interface. During assembly, the solvent-exposed side of the LSU is assembled first, characterized by folding of 5.8S rRNA with domains I and II of the 25S rRNA, and then with domain VI (Kater et al., 2017; Klinge \& Woolford, 2019; Sanghai et al., 2018). Meanwhile, the connecting domains III, IV, and VI remain flexible and bound by few AFs. These domains then begin to obtain structural stability and join the already folded domains I, II, and VI. Concomitantly, functional centers such as the PTC and PET start to assemble.

Early-acting AF have been successfully identified but their molecular functions are often poorly defined (reviewed in Baserga and Woolford). Among these include structural proteins with multiple RNA binding domains or alpha-helical repeats such as Rrp5, Mak21, Noc2 and Nop4. These proteins are proposed to function as rigid scaffolds to guide and stabilize RNA folding, necessary to reduce the conformational freedom of the pre-rRNA (Hierlmeier et al., 2013; Klinge \& Woolford, 2019; Lebaron et al., 2013; Sun \& Woolford, 1997). The requirement for RNA helicases, including Dbp2, Dbp3, Dbp6, Dbp7, Dbp9, Mak5, and Prp43, has also been uncovered, most likely to drive RNP structural rearrangements (Martin et al., 2013). Complexes of AFs, particularly the Npa1 complex consisting of Npa1, Npa2, Rsa3, Nop8, have also been reported to promote compaction of the central core in cooperation with Dbp6 (Joret et al., 2018). Additionally, r-proteins are also suggested to act as chaperones for folding and processing of pre-rRNA, not only in the early stages but all throughout of the biogenesis (reviewed in Jesus de la Cruz et al., 2015). The r-protein uL3 has been thought to mediate early pre-rRNA folding and compaction due to its contact points to both 5' and 3' end of the 25S rRNA (Ben-Shem et al., 2011). Furthermore, its depletion has the greatest destabilizing effect on very early pre-60S particles, indicative of its importance for pre-rRNA folding and subunit compaction (Gamalinda et al., 2014; Rosado et al., 2007b). Additionally, a recent study suggests a folding chaperone function of the orphan snoRNA snR190, which has multi-domain pre-rRNA targets in the domain $\mathrm{V}$ and I (Joret et al., 2018). The mechanistic details of how these AF, r-proteins, and snoRNA(s) promote folding and initial compaction is yet to be elucidated. 


\subsection{Removal of the ITS1 region}

As the pre-rRNA takes its early folding, its processing also commences. The A2 cleavage occurs once the transcription is approximately $1.2 \mathrm{~kb}$ downstream the A2 cleavage site, whereas the subsequent $A 3$ cleavage occurs when transcription is complete and the 3 ' ETS is processed (Allmang \& Tollervey, 1998; Kos \& Tollervey, 2010; Turowski \& Tollervey, 2015). The early pre-rRNA processing concludes with the generation of the 27SB pre-rRNA species devoid of ITS1.

The removal of ITS1 region also requires its dedicated AFs collectively called 'A3 factors'. This consists of 12 proteins namely Ebp2, Brx1, Pwp1, Nop12, Nop7, Ytm1, Erb1, Rlp7, Nop15, Cic1, Drs1, and Has1 (Adams et al., 2002; Dembowski et al., 2013b; Fabian \& Hopper, 1987; Fatica et al., 2003b; Gadal et al., 2002; Jäger et al., 2001; Oeffinger \& Tollervey, 2003; Shimoji et al., 2012; Talkish et al., 2014; Tang et al., 2008; Woolford \& Baserga, 2013). Most of these bind within or near ITS2 rather than within the ITS1 region (Granneman et al., 2011). Except for the DExD-box RNA helicase Drs1 and Has1, all of them lack enzymatic function. Moreover, depletion of these factors does not devoid the pre-LSU particles of the dedicated processing nucleases (Sahasranaman et al., 2011). All of these findings, together with recent cryogenic electron microscopy (cryo-EM) structures of pre-60S particles, point to these factors having a structural function necessary for the removal of ITS1. Furthermore, this suggests that early pre-rRNA folding and processing are tightly linked.

\subsubsection{Structural insights of nucleolar large subunit assembly}

Recent cryo-EM structures of nucleolar pre-60S particles (Kater et al., 2017; Sanghai et al., 2018; D. Zhou et al., 2019) provide us molecular snapshots of events occurring in early stages of LSU assembly. More importantly, they reveal how these rRNA secondary structure domains are folded in 3D space and held together by assembly factors and r-proteins to maintain compact and stable early intermediates.

The earliest structures (State A-C or State 2) of pre-60S particles present an open conformation of the already assembled domains I, II, and VI (Kater et al., 2017; Sanghai et al., 2018) (Figure 5A; Figure 5B). This architecture is maintained by the association of several assembly factors. Brix-domain-containing factors Brx1, Rpf1, and Ssf1, together with their binding partners (Ebp2, Mak16, and Rrp15), bridge these domains and provide steric hindrances to prevent premature RNA-protein and RNA-RNA interactions (Sanghai et al., 
A

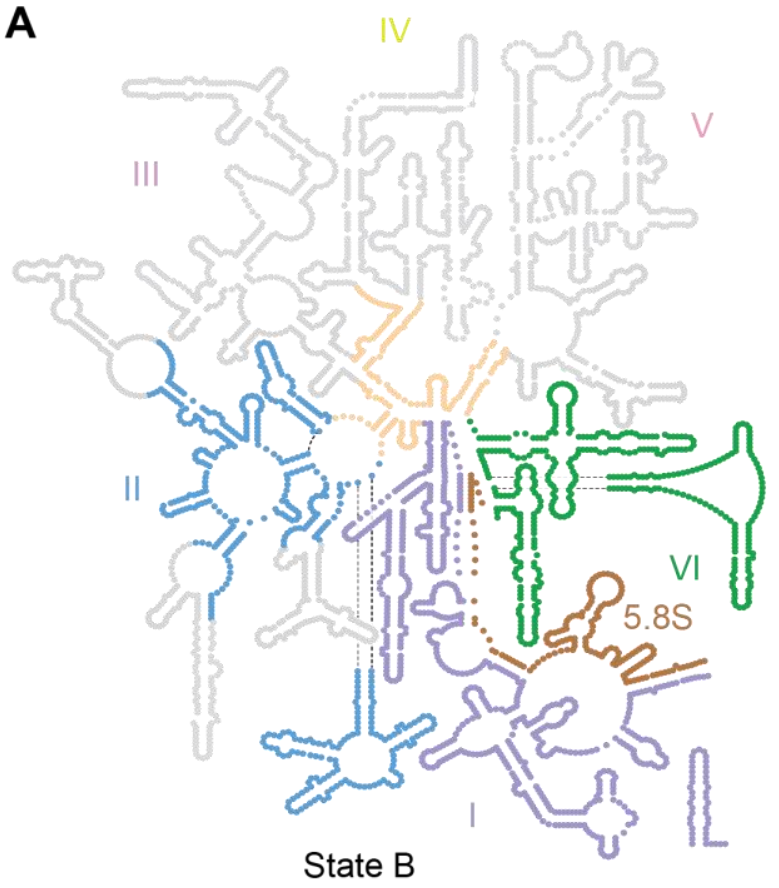

C

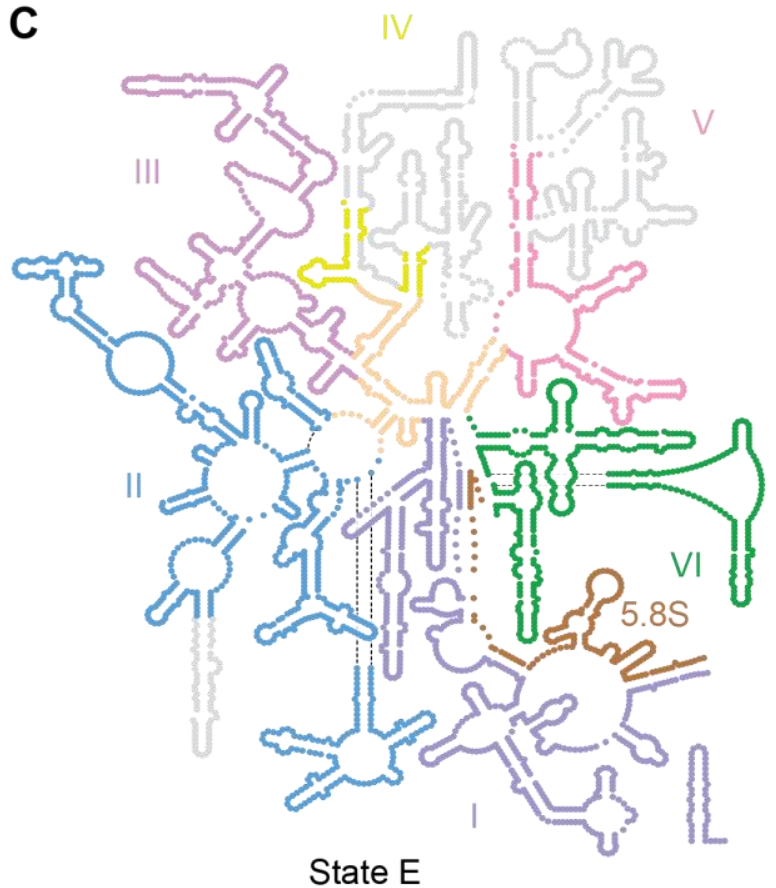

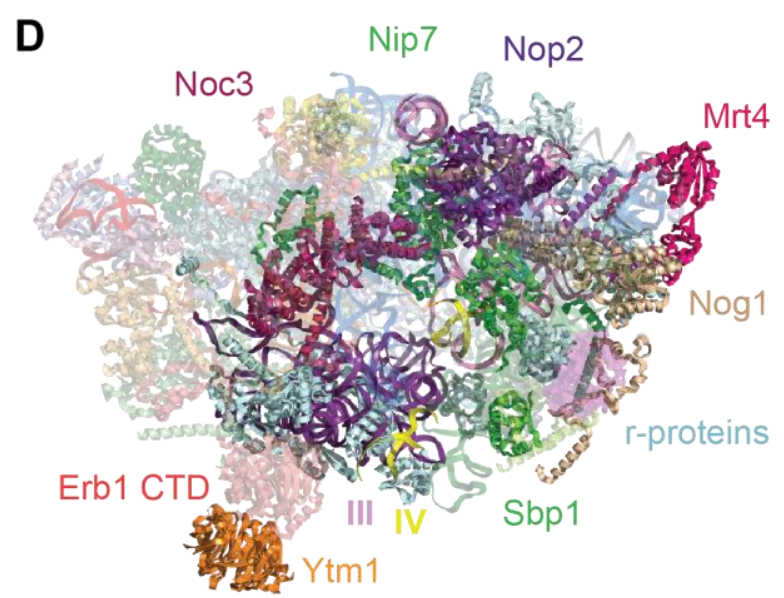

B

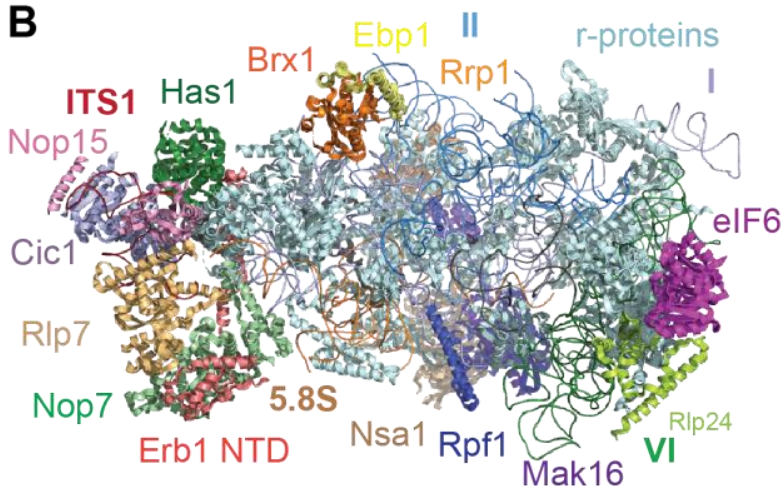

Figure 5. Nucleolar assembly of large subunit. (A) Pre-rRNA folding in the so-called state B pre60 S purified via TAP-tagged Nsa1 followed by Flag-tagged Ytm1. rRNA domains I (purple), II (blue), and $\mathrm{VI}$ (green) show stable folding and organization early on, while the other domains (shown in grey) remain disordered. Schematic diagrams including that were generated based on the available secondary structure map (Petrov et. al., 2014a) (B) Reconstructed cryo-EM structure of state B nucleolar pre-60S particle. Early binding AF are re-colored as indicated while all r-proteins are represented as cyan. rRNA domains with structural information are represented as in A. Cryo-EM structure of state B (PDB ID:6COF) was reconstructed form Kater et. al. (2017) (C) Pre-rRNA folding in the so-called state E pre-60S. Successive folding of domains III (violet) and parts of domain IV (yellow) and V (pink) is observed. (D) Reconstructed cryo-EM structure of state $E$ nucleolar pre-60S (PDB ID: 6ELZ) based from structural data of Kater et. al. (2017). Additional AFs recruited in this later state were overlaid and shown in different colors as indicated. Additional RNA structure resolved were also represented with color scheme similar to (C). Diagrams constructed based on Kater et al. (2017), Sanghai et al. (2018), and Klinge and Woolford (2019). 
2018). Rfp1 and its zinc-binding partner Mak16 act as the central scaffold between the major interface of domains I and II while additional structural support is provided by the Brx1-Ebp2 heterodimer, Nsa1 and Rrp1 (Kater et al., 2017). Meanwhile, the interface of domain VI is held by C-terminal helix of Rrp14, which then bridges domain VI and domain II (Sanghai et al., 2018). Additionally, other factors (the GTPase Nog1, elF6, Rlp24 and Mak5) assemble with domain VI near the interface with domain V. Interestingly, structural data reveal the positions of r-proteins implicated in the ribosomopathy Diamond- Blackfan anemia to be in critical rRNA domain interfaces, substantiating their role in early assembly and the nucleolar stress observed during the onset of the disease (Sanghai et al., 2018). Meanwhile, the "A3 factors" Nop15, Cic1 and RIp7 bind to ITS2 to form early on the "foot" structure typically seen in later particles isolated via Nog2 or Arx1 (Granneman et al., 2011; Wu et al., 2016). Other "A3" factors (Nop7, the Erb1-Ytm1 complex and Has1) together with Nop16 are positioned in domain I surrounding ITS2. Together with several r-proteins around this region, these "A3" factors are anticipated to hold the early secondary structures formed from base pairing of $5.8 \mathrm{~S}$ and parts of domain I, and consequently stabilize the tertiary folds. The middle stage B-factors such as Nog1, Mrt4, Tif6, and Rlp24 are also already present.

In later nucleolar pre-60S intermediates (State 3 or D-E), the loosely tethered domains III, IV, and $\mathrm{V}$ start to fold, shifting the overall architecture of the particle into a globular conformation similar to the mature 60S (Figure 5C; Figure 5D). Central to this conformational change is the acquisition of several AFs, namely Nip7, Noc3, Nop2, Sbp1 and Ytm1, as well as the stable incorporation of addition r-proteins. In these later states $D$ and $E$, the role of Erb1 as an interaction center for a number of AFs (Brx1, Ebp2, Noc3, Has1, Nop16, Nop7, Rlp7, Ytm1) and few r-proteins (eL27, eL36, eL38) was deduced and this in turn is key in facilitating the coordination of domains I, III, and VI (Kater et al., 2017). Additionally, structural data also revealed that the N-terminal of Erb1 binds proximal to Nop7 and mimics the binding of Nop53 (Sanghai et al., 2018). This prevents association into the pre-60S particle of Nop53, which is necessary for the recruitment of exosome-associated RNA helicase Mtr4 for ITS2 processing (Thoms et al., 2015). In terms of functional cores, the folding of domains III and parts of domain IV and V leads to the formation of the PET and tunnel exit site in these pre-60S states. The L1 stalk remains in a premature state until later state $F$, where additional folding of domains IV and $\mathrm{V}$ and the rotated of $5 \mathrm{~S}$ RNP provide additional structural features to the developing subunit interface and immature CP (Kater et al., 2017; Klinge \& Woolford, 2019; Wu et al., 2016).

These molecular snapshots of early stage pre-60S particles present key concepts of eukaryotic ribosome biogenesis. First, there is evident hierarchical incorporation of most $r$-proteins 
consistent with what has been suggested previously (Gamalinda et al., 2014). Similarly, AFs are recruited in a chronological manner and associate only for a particular span of time. This presents a high degree of assembly regulation through mechanisms recently coined as 'molecular mimicry' and 'molecular switching' to prevent premature rRNA-rRNA and rRNAprotein interaction as well as untimely pre-rRNA processing (Klinge \& Woolford, 2019; Sanghai et al., 2018). Additionally, the timely removal of AFs is also as important as their presence and this process is usually driven by energy-dependent irreversible conformational changes proving directionality of the process. Furthermore, the assembly pathway occurs in a modular fashion, similar to prokaryotic 50 S biogenesis (Davis et al., 2016). However, in contrast to bacteria, the construction of domains is non-linear and does not follow the order of transcription. Moreover, the different structural states argue against the existence of parallel pathways of assembly as observed in bacteria (Davis et al., 2016). This is possibly due to the presence of numerous AFs in eukaryotes to orchestrate the assembly in an ordered and sequential manner.

\subsection{RNA helicases}

RNA helicases are highly conserved enzymes that structurally remodel RNA molecules and RNP complexes into different conformations. These enzymes typically unwind RNA duplexes in an energy-dependent manner through NTP binding and hydrolysis (Bleichert \& Baserga, 2007; Cordin et al., 2006; Jarmoskaite \& Russell, 2014). In addition to their classical unwinding function, some RNA helicases have been shown to promote dissociation of RNA-protein interactions, anneal and exchange RNA strands, act as scaffolds for protein-protein interactions, function as RNA clamps, and stabilize RNA substructures (Bleichert \& Baserga, 2007; Gilman et al., 2017; Linder \& Jankowsky, 2011; Owttrim, 2013). RNA helicases are involved in many key cellular pathways and this wide spectrum of functions explains their ubiquitous nature and requirement for cell viability.

\subsubsection{Classification and Structure of RNA helicases}

All helicases involved in RNA and DNA metabolism are classified into six different superfamilies (SF1-6) based on sequence, structure and function (Gorbalenya \& Koonin, 1993; Singleton et al., 2007). SF1 and SF2 helicases are monomeric in nature whereas helicases belonging to SFs 3 to 6 form hexameric rings (Singleton et al., 2007). Most RNA helicases are members of SF2 superfamily and only a small number belong to SF1 (Bleichert \& Baserga, 2007; Jankowsky, 2011) (Figure 6A). RNA helicases belonging to the SF2 superfamily are mainly found in five subfamilies (DExD Box, DEAH/RHA box, Ski2-like, RIG-l-like, NS3/NPHII) with majority of them classified under DExD box and DEAH box subfamilies. 


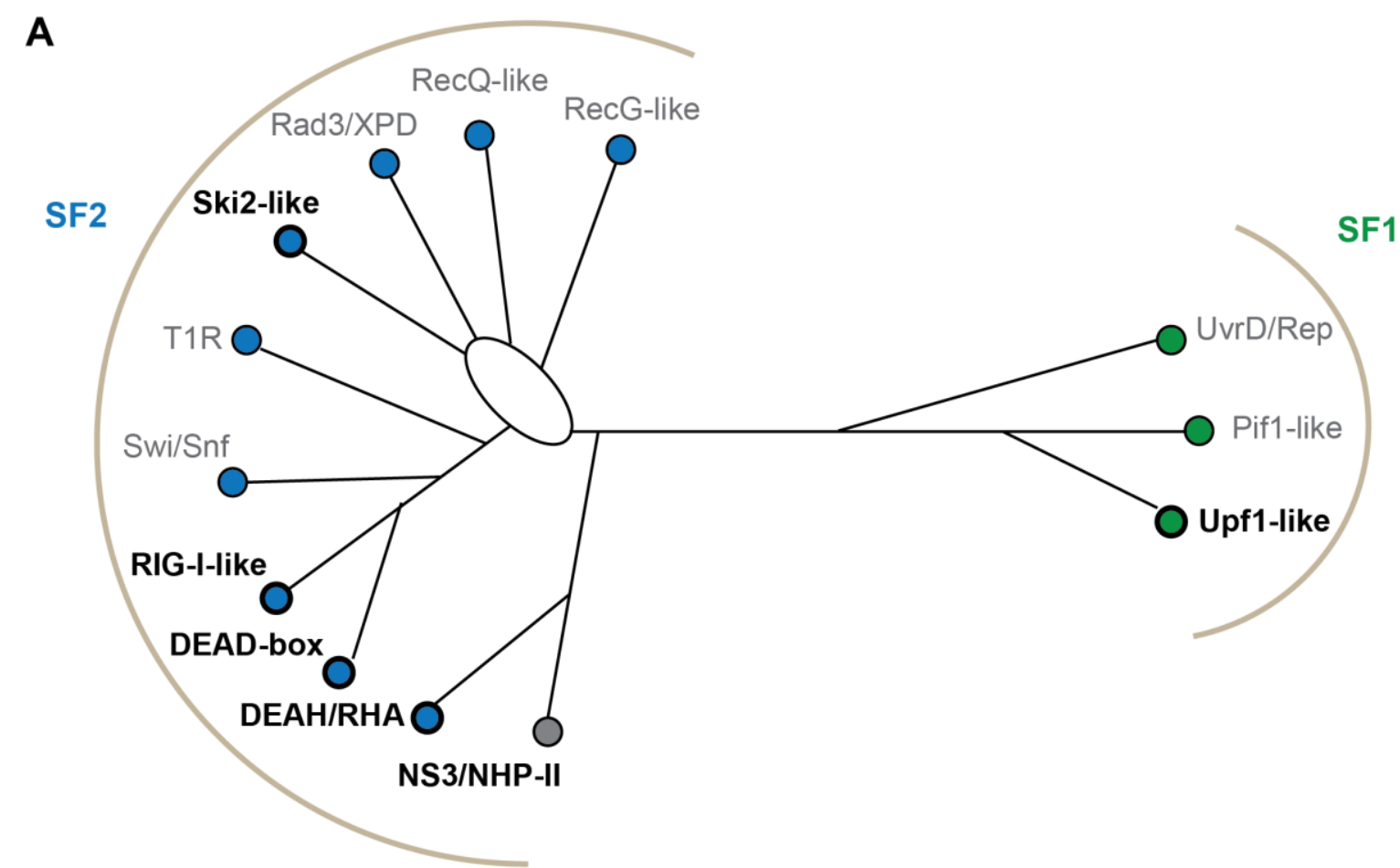

B

Helicase core

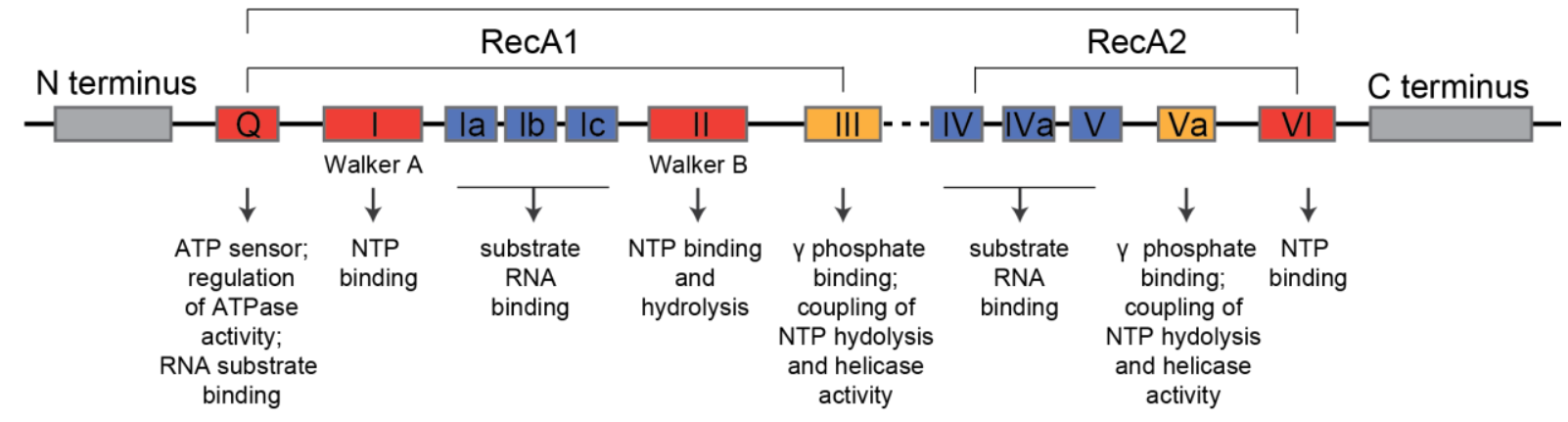

Figure 6. Classification and characteristics of SF1 and SF2 superfamily RNA helicases. (A) Cladogram of SF1 and SF2 with their subfamilies. RNA helicases cluster in 6 subfamilies shown in bold. This diagram is adapted from Fairman-Willaims (2010) and Jankowsky (2011) with branch length not to scale. (B) Schematic diagram of the conserved helicase core motifs of DExD/H RNA helicases. The helicase core consists of two RecA-like domains, RecA1 and RecA2, which contain conserved sequence motifs responsible for NTP binding and hydrolysis (red), RNA binding (blue), and coupling of NTP binding and hydrolysis (yellow). The Q motif is only found in DExD box helicases. In some cases, auxiliary regions in $\mathrm{N}$ - and C-terminal (gray) are present. Diagrams adapted and modified from Bleichert \& Baserga (2007) and Linder and Jankowsky (2011).

Members of the SF1 and SF2 superfamilies possess a highly conserved catalytic core with highly identical structure. RNA helicases belonging to these two superfamilies harbor a helicase core consisting of two tandemly repeated RecA-like domains called RecA1 and RecA2 (Figure 6B). These two domains form a cleft where NTP can bind on one side and the RNA substrate on the other side. NTP binding brings the two domains into a closed conformation, which is converted back into an open conformation upon NTP hydrolysis (Jarmoskaite \& Russell, 2014). To facilitate this enzymatic and mechanical function, the two 
domains contain up to 12 conserved sequence motifs responsible for RNA-substrate binding, and NTP-binding and hydrolysis (Fairman-Williams et al., 2010). Motifs I (Walker A motif), II (Walker B motif), and VI are involved in NTP binding and hydrolysis (Bleichert \& Baserga, 2007; Ranji \& Boris-Lawrie, 2010). More specifically, motif I contains a glycine-lysine-threonine (GKT) amino acid sequence, which is conserved in SF1 and SF2 RNA helicases and is directly implicated in NTP binding (Jankowsky \& Fairman-Williams, 2010). The side chain of the lysine residue interacts with the beta-phosphate of the NTP to hold the NTP in proper orientation (Linder \& Jankowsky, 2011; Pause \& Sonenberg, 1992). Motif II harbors conserved glutamic acid and aspartic acid residues. The glutamate coordinates the catalytic water and act as a catalytic base necessary for NTP hydrolysis while the aspartate coordinates magnesium ions (Caruthers \& McKay, 2002; Linder \& Jankowsky, 2011). Motif III has been proposed to coordinate the NTP hydrolysis state with the helicase activity in some RNA helicases (Cordin et al., 2006; Dillingham et al., 1999; Linder \& Jankowsky, 2011). Motif VI is also involved in NTP hydrolysis by stabilizing the transition state through conserved arginine residues (Jankowsky \& Fairman-Williams, 2010; Pause et al., 1993; Pause \& Sonenberg, 1992). The Q motif, recently identified and present only in DExD box helicases, renders specificity for adenosine triphosphate (ATP) over other NTPs and has been proposed to regulate ATPbinding and hydrolysis (Cordin et al., 2004; Sloan \& Bohnsack, 2018; Tanner et al., 2003). Other motifs (Ia, Ib, Ic, IV, IVa and V) remain to be fully characterized, however, they are predicted to bind substrate RNA and/or bridge the RNA-binding site to the NTP-binding site (Banroques et al., 2008; Cordin et al., 2006; Jankowsky \& Fairman-Williams, 2010).

With the exception of the minimal RNA helicases belonging to elF4A-like group, most RNA helicases have flanking $\mathrm{C}$ - and N-terminal domains, which can be larger in size than the helicase core and have diverse functionalities. They can enable interactions with other proteins or facilitate the targeting of helicases to its RNA substrates and hence, endow target specificity (Karginov et al., 2005; Lattmann et al., 2010; Linder \& Jankowsky, 2011). Interaction with other proteins can increases substrate repertoire and allows multifunctional RNA helicases to be directed to different cellular functions (Heininger et al., 2016). Additionally, these auxiliary domains can fold to form structures with functionalities such nuclease activity, as well as to drive oligomerization (Donsbach et al., 2020; Hardin et al., 2010; Klostermeier \& Rudolph, 2009; Rudolph \& Klostermeier, 2009, 2015; Yoneyama \& Fujita, 2008).

\subsubsection{DExD/H proteins as RNA and RNP chaperones}

The DExD and DEAH box families, collectively called DExD/H box proteins, have the largest number of RNA helicase that remodel RNA structure and RNP complexes (Jarmoskaite \& Russell, 2014; Tanner \& Linder, 2001). These two subfamilies consist of a variety of RNA 
helicases with sizes ranging from 400 to more than 2000 amino acid residues (Bleichert \& Baserga, 2007; Martin et al., 2013; Tanner \& Linder, 2001). Despite the heterogeneity in size and sequence, proteins belonging to these two families harbor an evolutionary conserved classical helicase core. DExD Box RNA helicases are characterized by the presence of a conserved amino acid sequence, aspartate-glutamate-X-aspartate, within motif II of domain I whereas DEAH box proteins harbor conserved aspartate-glutamate-alanine-histidine residues. In addition to these characteristic sequence differences, proteins belonging to these two families differ significantly in their mechanisms of substrate binding and unwinding (Figure 7).

\subsubsection{The translocating DEAH RNA helicases}

The DEAH box RNA helicases structurally differ from the DExD RNA helicases in their terminal auxiliary domains. In particular, DEAH RNA helicases share a conserved C-terminus consisting of three domains namely the $\mathrm{OB}$ (oligosaccharide binding) fold, winged-helix and ratchet domains (Gilman et al., 2017; Ozgur et al., 2015). Structural data revealed a strong physical interaction of these C-terminal domains with the helicase core, resulting in a more rigid structure compared to DExD box RNA helicases (He et al., 2010; Murakami et al., 2017; Pyle, 2008). Moreover, these C-terminal extensions form an RNA-binding tunnel, which is structurally rearranged by NTP binding to allow incorporation of the RNA substrate (Tauchert et al., 2017).

Similar to many DNA helicases, DEAH box RNA helicases, together with Ski2-like, RIG-I-like, and Upf1-like helicases, display translocation-based duplex unwinding (Jarmoskaite \& Russell, 2014; Lohman \& Bjornson, 1996). They are highly processive, directional (3' to 5') and can unwind long RNA duplexes (Gilman et al., 2017). Additionally, they require 3' single-stranded extensions, which serve as loading platforms (Bernstein et al., 2006; Tanaka \& Schwer, 2005) and can bind and hydrolyze all four NTPs as energy source (Fairman-Williams et al., 2010). In contrast to DExD box helicases, many DEAH box proteins can also unwind DNA substrates, including four-stranded G-quadruplexes (M. C. Chen et al., 2015, 2018).

Recent structural and biochemical data on the splicing-associated RNA helicases Prp43 and Prp22 from Chaetomium thermophilum provided mechanistic models of translocation-based unwinding of DEAH box proteins (Hamann et al., 2019; Tauchert et al., 2017). In the absence of ATP/ADP, the RecA-like domain RecA2 has intrinsic mobility that presents an open conformation. In this ATP-free open state, the helicase core can accommodate five nt of the bound RNA between a long beta-hairpin in RecA2 and a hook-turn loop in RecA1. Upon binding of ATP, to promote the closed state, RecA2 moves towards RecA1, pushing the singlestranded RNA in the 3' direction with a single nt distance. During the transition from the closed 
to open state, the beta-hairpin acting as a physical barrier in RecA2 is then shifted allowing the incorporation of the next nucleotide at the $5^{\prime}$ end (Figure 7A). Hence, DEAH box helicases translocate along single stranded RNAs in the 3' to 5' direction in a step-wise manner with a rate of one nt per hydrolyzed ATP (Hamann et al., 2019). This directional translocation facilitates RNA duplex unwinding by disruption of encountered base-pairing, as well as the 'winching' mechanism described for some DEAH box RNA helicase to globally remodel RNA and RNP complexes especially in splicing (Semlow et al., 2016).

\subsubsection{DExD box RNA helicases as local 'unwindase'}

The DExD Box RNA helicases make up the largest family of the SF2 helicases, consisting of 37 members in humans and 26 in S. cerevisiae (Fairman-Williams et al., 2010; Jarmoskaite \& Russell, 2014). These enzymes all share highly conserved tandem RecA-like domains bridged by a short flexible linker and containing 13 conserved sequence motifs (Del Campo \& Lambowitz, 2009; Mohr et al., 2011). Majority of DExD box RNA helicases do not harbor terminal auxiliary domains aside from the helicase core although their $\mathrm{N}$ - and $\mathrm{C}$-terminal flanking regions are in some cases very long (Jankowsky \& Fairman-Williams, 2010). Early investigations with the translation initiation factor elF4A highlighted the non-processive nature of unwinding of DExD box proteins with a typical unwinding capacity of up to only $15 \mathrm{bp}$ (Rogers et al., 1999). Additionally, several studies have shown that DExD box proteins can unwind using a single cycle of ATP binding and hydrolysis, and unwind substrates without overhanging 3' or 5' extensions (Bizebard et al., 2004; Y. Chen et al., 2008; Henn et al., 2010; Rogers et al., 2001; Yang \& Jankowsky, 2006). Furthermore, unwinding is not directional and is possible without ATP hydrolysis (F. Liu et al., 2008; Yang \& Jankowsky, 2006). With these lines of evidence, DExD box RNA helicases are considered to unwind RNA duplexes by local strand separation (Figure 7B).

More mechanistic insights were provided by studies on the yeast mitochondrial DExD-box Mss116 (Mallam et al., 2012). Biochemical and structural data revealed that the RecA2 domain alone can bind double-stranded RNA (dsRNA) while RecA1 independently binds ATP. The cooperative binding of these two ligands, first ATP and then the dsRNA, switches the conformation of the two RecA-like domains from an open conformation to a close active state (Andersen et al., 2006; Mallam et al., 2012; Theissen et al., 2008). In this close conformation, a conserved alpha-helix in the RecA1 domain is extruded and consequently introduces a kink in one RNA strand that disrupts base-pairing and consequently releases the other strand while the bent strand remains bound to the complex (Andersen et al., 2006; Ozgur et al., 2015; Sengoku et al., 2006). Hence, DExD box RNA helicase display a non-conventional unwinding mechanism that involves a non-processive, ATP-dependent distortion of one RNA strand that 
then stochastically separates adjacent base-pairing. Additionally, closure of the domains triggers ATP hydrolysis and release of inorganic phosphate, which in turn releases the bound RNA strand and allows recycling of the enzyme (Y. Chen et al., 2008; Hilbert et al., 2009; F. Liu et al., 2008).

A

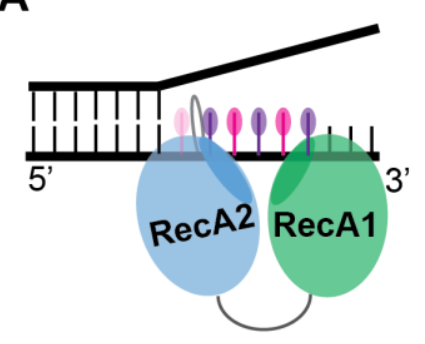

B

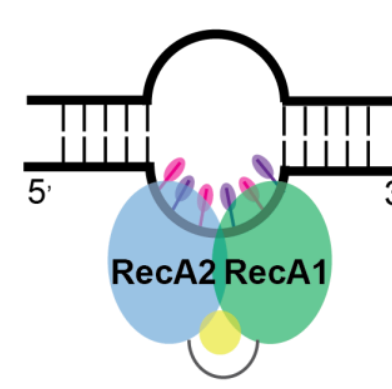

helicase loading
ATP Binding

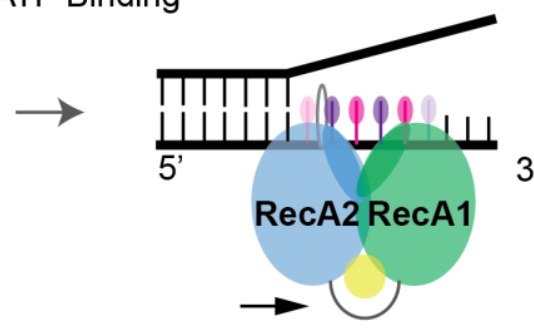

ATP hydrolysis

(1)

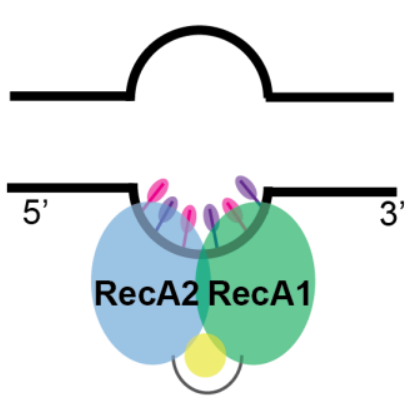

strand separation

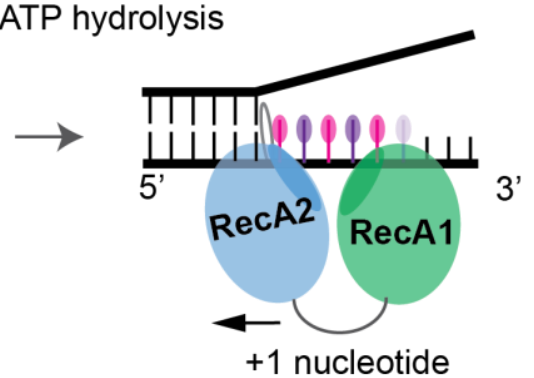

ATP hydrolysis

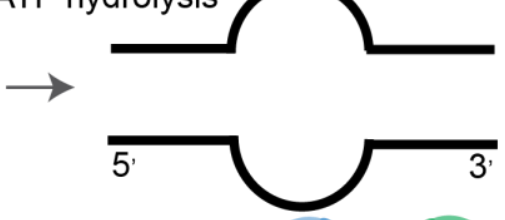

$\operatorname{Rec} A 2 \operatorname{Rec} A 1$

helicase disociation

Figure 7. Schematic diagram of the unwinding mechanisms of DEAH and DExD box RNA helicases. (A) Translocation model of DEAH RNA helicases with a rate of one nt per hydrolyzed ATP. Nucleotides within the cleft formed by RecA1 (green) and RecA2 (blue) are colored in purple and pink. Closing (four nt) and opening (five nt) of RecA1 and RecA2 domains upon ATP (yellow) binding and hydrolysis, respectively, are indicated by black arrow as the helicase translocate from 3' to 5' direction. Diagram constructed based on Hamann et. al., (2019). (B) Local unwinding mechanism proposed for DExD box RNA helicases. ATP-bound closed conformation sequesters six nt within the RNA binding cleft resulting to the distortion in strand and consequent disruption of baseparining interactions. RNA helicase is released from one strand upon ATP hydrolysis and release of inorganic phosphate. Diagram constructed based on Gilman et al. (2017) and Ozgur et al. (2015)

While a non-translocation mechanism can effectively unwind short RNA duplexes, longer duplex would likely require multiple rounds of binding, unwinding and ATP hydrolysis. This can be facilitated by the presence of $\mathrm{C}$ - or $\mathrm{N}$-terminal auxiliary domains that are thought to help the enzyme anchor itself to the target region (Grohman et al., 2007; Mohr et al., 2011; Talwar et al., 2017). Interestingly, some DExD box RNA helicases, such as Ded1 and Mss116, can promote strand annealing and RNA duplex formation (Uhlmann-Schiffler et al., 2006; Yang \& Jankowsky, 2006). Additionally, as exemplified by the exon junction complex (EJC) core component elF4A-III, DExD box RNA helicases can also act as RNA clamps by stabilizing the 
ADP-Pi-bound state through interaction with other protein (Ballut et al., 2005; Linder \& Jankowsky, 2011).

\subsubsection{Regulation of RNA helicases}

Structural data revealed that RNA helicases interact mainly with the sugar-phosphate backbone and therefore, lack intrinsic specificity to their target RNA (Linder \& Jankowsky, 2011; Ozgur et al., 2015). Furthermore, several RNA helicases demonstrate very low catalytic rates (Andersen et al., 2006; Jarmoskaite \& Russell, 2014; Sengoku et al., 2006). Given these properties, the cell has developed different means to control the localization/distribution, timing and activity of RNA helicases to employ them efficiently. Recently, several mechanisms of regulation involving accessory proteins, post-translational modifications, non-coding RNAs, and substrate RNA properties have been documented (reviewed in Sloan \& Bohnsack, 2018). Among these, accessory proteins, also called 'cofactors', have emerged as key factors in RNA helicase regulation. These cofactors could stimulate ATPase and helicase activity, recruit a helicase to its specific substrate(s) and/or increase the affinity of the helicase for its substrate, or inhibit helicase activity (Cordin et al., 2006; Martin et al., 2013; Sloan \& Bohnsack, 2018). While a few are unrelated group of proteins (stand-alone cofactors), many share common domains that serve as binding platforms for their target RNA helicases. To date, two families, namely the MIF4G family and G-patch proteins, have emerged as dedicated regulatory proteins of DExD and DEAH RNA helicases respectively.

The MIF4G family of cofactors are characterized by the presence of an evolutionarily conserved MIF4G (Middle domain of elF4G) domain consisting of ten anti-parallel alpha helices that folds to form an arc shape (Ponting, 2000). Structural studies of DExD cofactorshelicase interaction, i.e. elF4A1 and elF4G, revealed that the $\mathrm{N}$ - and C-terminal of MIF4G domain interacts strongly with the RecA2 and RecA1 respectively, leading to the formation of a 'half-open' conformation that promotes RNA binding and accelerates the rate-limiting phosphate release (Andreou \& Klostermeier, 2014; Hilbert et al., 2011; Schütz et al., 2008). Additionally, single molecule analyses revealed the possibility of elF4Al to convert its unwinding to a processive manner, typical of DEAH box RNA helicases, in the presence of its cofactors elF4G and elF4A (García-García et al., 2015). In contrast, members of G-patch harbor a glycine-rich stretch of 40-50 amino acid called G-patch domain, which is defined by

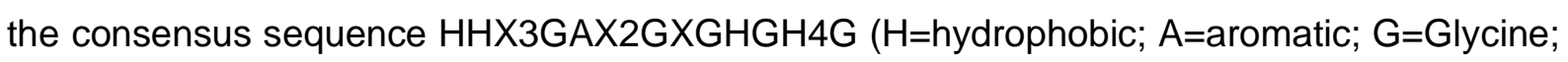
$X=$ non-conserved) (Aravind \& Koonin, 1999). Recent structural investigations revealed that the highly unstructured G-patch domain consisting of an N-terminal alpha helix (brace helix), a linker region, and a C-terminal brace loop wraps around the back side of the RNA-binding tunnel all the way to the RecA2 domain (Hamann et al., 2020; Studer et al., 2020). This involves 
hydrophobic interactions between (1) the winged-helix c-terminal domain and the amphipathic brace helix, and (2) the brace loop and RecA2 domain. Furthermore, the highly-conserved glycine residues promote a topology of the G-patch domain necessary for these contact points (Hamann et al., 2020). As a consequence, these protein interactions bring the two RecA domains closer to produce a conformation conducive for high affinity RNA binding and ATP hydrolysis rate. These findings led to a model proposing that free RNA helicase remains flexible until it encounters its dedicated G-patch protein, which then reduces its flexibility and simultaneously recruit the RNA helicase to its RNP substrate (Studer et al., 2020). This reduction of flexibility results to a high RNA affinity configuration, a mechanism highly identical to MIF4G-domain containing protein. However, in the case of G-patch proteins, they also prevent the opening of the RNA binding tunnel after ATP hydrolysis, thereby maintaining the grip on RNA necessary for translocation.

Other modes of co-factor-mediated regulation of RNA helicases have also been documented. These include mechanisms that positively (e.g. MLN51 to elF4AII) or negatively (e.g. Pat1 and Edc3 to Dhh1) affects propensity of the helicase core for substrate RNA binding (Noble \& Song, 2007; Sharif et al., 2013). Likewise, other types of domains that facilitates interaction with RNA helicases are being uncovered. For example, the OST-HTH/eLOTUS domain found in some proteins including the co-factors of the RNA helicase Vasa and was shown to bind only the RecA2 domain and consequently reorients motif $\mathrm{V}$ and the QxxR motifs upon switching from open and close conformation (Jeske et al., 2017). More co-factors are expected to be identified and characterized in the coming years.

\subsubsection{Roles of RNA helicases in different cellular pathways}

Due to their intrinsic ability to reorganize RNA structures and RNP complex coupled with the importance of proper RNA structure for biological processes, RNA helicases are implicated in most aspects of RNA metabolism starting from transcription until degradation of RNAs. Furthermore, RNA helicases are important for a number of molecular machines, such as spliceosomes and (pre-)ribosomes, whose assembly and function involve various conformational changes.

Ribosome biogenesis is the cellular process that involves the most number of RNA helicases (details in section 1.3.5). This is then followed by pre-mRNA splicing where eight RNA helicases (Prp16, Prp2, Prp22, Prp5, Prp28, Brr2, Prp43) are implicated in yeast. These helicases mediate RNA secondary structure rearrangement, protein removal and intermediate structure stabilization necessary for the fidelity and directionality of the splicing process (Cordin \& Beggs, 2013; Y.-C. Liu \& Cheng, 2015). Moreover, RNA helicases, such as Mss116/CYT- 
19, are also involved in self-splicing group I and II introns (Huang et al., 2005). Other RNA metabolism pathways also require RNA helicases. For example, following the splicing process, mature mRNAs are exported through the NPC and this transport requires the DExD box RNA helicase Dbp5. Genetic studies suggest that Dbp5 is required for the release of Mex67, an essential step during the mRNA export (Lund \& Guthrie, 2005). After export, the mRNA is decoded by the ribosome to produce cellular proteins. The initial step of the translation process entails scanning for the start codon by the pre-initiation complex (PIC) consisting of the $40 \mathrm{~S}$ and numerous initiation factors. This is efficiently done with the aid of the DExD box RNA helicase elF4A and other initiation factors, e.g. elF4G, to dissolve secondary structures encountered at the 5' untranslated region (UTR) (Aitken \& Lorsch, 2012; Svitkin et al., 2001). Another more potent RNA helicase Ded1 is also involved and reported to regulate translation initiation (Hilliker et al., 2011). mRNAs and other RNAs have limited lifespan in the cells and have to be degraded. The degradation of RNA employs the nuclear and cytoplasmic exosomes with RNA helicases Mtr4 and Ski2 respectively, as one of their associated factors. These RNA helicases unwind structured RNAs and propel single stranded RNAs into the degradation machinery (Halbach et al., 2013; Jia et al., 2012). Aside from these key cellular pathways, many other biological processes employ the chaperone activities of RNA helicases. For example, cellular defense against viral infection employs RIG-I-like receptors ( $R L R$ ) belonging to SF2 families to recognize viral RNAs (Fitzgerald et al., 2014; Luo et al., 2011). Aside from the conserved RecA-like domains, these RNA helicases have evolved accessory domains that allow recognition of viral-specific RNA duplexes and binding of effector proteins to mount antiviral defenses (Rawling \& Pyle, 2014). Altogether, these demonstrate the arsenal of physiological roles of RNA helicases.

\subsubsection{RNA helicase implicated in yeast ribosome biogenesis}

Ribosome biogenesis is one of the key cellular pathways that requires the remodeling and chaperoning activities of RNA helicases. In yeast, 21 RNA helicases are implicated in ribosome biogenesis, most of which are DExD-box proteins (Martin et al., 2013). Eight RNA helicases (Dbp4, Dbp8, Dhr1, Dhr2, Fal1, Kre33, Rrp3, Rok1) are involved in SSU biogenesis, while eleven (Dbp2, Dbp3, Dbp6, Dbp7, Dbp9, Dbp10, Drs1, Mak5, Spb4) have roles in the production of LSU, and the RNA helicases Prp43, Dbp5, Mtr4 and Has1 function in the synthesis of both subunits (Figure 8). They can be further broadly classified as early- or lateacting based on the pre-rRNA processing phenotype observed and ribosome intermediates that accumulate upon their depletion (reviewed in Martin et al., 2013). With the exception of Dbp2, Dbp3 and Dbp7, all are essential for cell viability reflecting their crucial function. In general, their remodeling functions play important roles in the folding, processing, and modification of pre-rRNAs, as well as in inducing changes in protein composition of pre- 
ribosomal particles during the course of subunit assembly. To date, knowledge of the precise molecular functions of many of these RNA helicases remains limited.

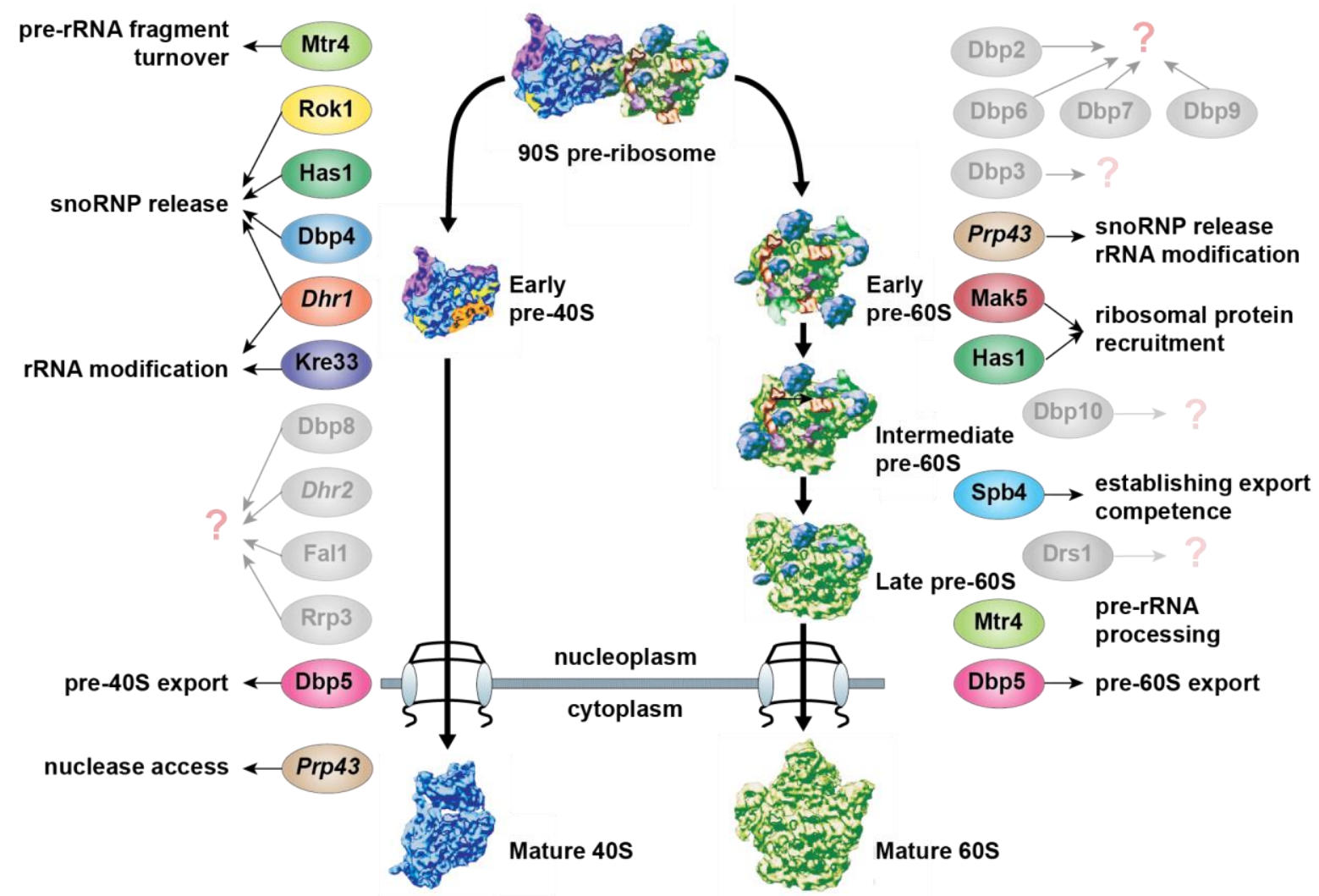

Figure 8. Functional classification of DExD/H RNA helicases involved in yeast ribosome biogenesis. RNA helicases are listed on the side to indicate the division of labor between the SSU and LSU biogenesis. Italicized are the DEAH box helicases while the rest are DExD box helicases except for the Ski2-like RNA helicase Mtr4. Shown in colored are RNA helicases for which insights into their role in the assembly process have been reported. Shown in light grey are RNA helicases which functions remain to be elucidated. Diagram modified from Martin (2014) PhD thesis.

\subsubsection{RNA helicases regulate snoRNA-pre-rRNA interactions}

Extensive functional characterization of the DExH RNA helicase Prp43 elegantly provides insights into the role of RNA helicases in regulating snoRNP dynamics in pre-ribosomes. This multi-functional RNA helicase has been shown to have a role in the release of several snoRNAs (snR39, snR39b, snR50, snR59, snR60, and snR72) from the pre-ribosome (Bohnsack et al., 2009). In vivo UV crosslinking data revealed that Prp43 directly interacts with these snoRNAs and also crosslinks to the regions where the modification guided by these snoRNA are clustered in the pre-rRNA. Notably, Prp43 also crosslinks close to the 3 ' end of the $18 \mathrm{~S}$ rRNA, and physically and genetically interacts with Nob1, indicating a role in promoting Nob1-mediated 3' cleavage of the 20S pre-rRNA to generate the mature 18S rRNA (Bohnsack et al., 2009; Pertschy et al., 2009). Aside from releasing, Prp43 has also been shown to be 
necessary for pre-ribosomal association of snR64 and snR67, which further supports the suggested role of Prp43 in regulating pre-ribosomal snoRNA dynamics (Bohnsack et al., 2009; Leeds et al., 2006). Similarly, the acetyltransferase-helicase Kre33 has been also reported to promote the annealing of its guide snR4 and snR45 to the pre-rRNA target site (Sharma et al., 2017).

Aside from Prp43, the role of Dbp4, Has1, Rok1 and Dhr1 in the release of snoRNAs from preribosomal particles has been described. The DExD-box RNA helicase Dbp4 is required for the removal of snoRNAs U14 and snR41 (Kos \& Tollervey, 2005). Although depletion of Dbp4 leads to accumulation of $\mathrm{U} 14$ in the pre-ribosome, it is not directly implicated in mediating the release of U14 (Soltanieh et al., 2015). Similarly, the role of Has1 in releasing U3 and few other snoRNAs except U14 are indirect (Liang \& Fournier, 2006). For U14, in vivo crosslinking

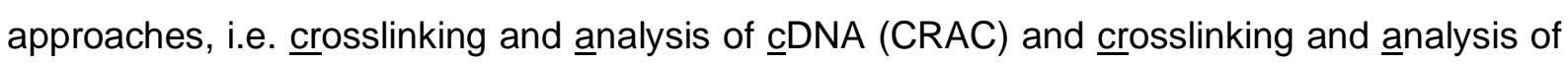
sequence hybrids (CLASH), revealed binding of Has1 to U14 and to the baseparing site of U14 on the 25S rRNA (Brüning et al., 2018). This, therefore, suggests a direct role of Has1 in the release of U14. Meanwhile, Rok1 has been implicated in the release of an essential snR30 as evidenced by pre-ribosomal accumulation of snR30 upon Rok1 depletion (Bohnsack et al., 2008). CRAC and CLASH data also suggest a direct role of Rok1 in the unwinding of snR30$18 S$ duplex (Martin et al., 2014). The DExD box RNA helicase Dhr1 is implicated in the dissociation of $\mathrm{U} 3$ to drive the formation of the central pseudoknot of $18 \mathrm{~S}$ and this function has been shown to be conserved in humans (Choudhury et al., 2019; Sardana et al., 2015).

\subsubsection{RNA helicases promote structural changes in pre-ribosomes}

Remodeling function of several RNA helicases have been shown to promote pre-rRNA processing and recruitment/release of r-proteins and AFs. For example, unwinding of rRNA secondary structure mediated by Ski2-like helicase Mtr4 is necessary for 5.8S rRNA and 3' ETS processing by the nuclear exosome (Falk et al., 2017; Schuller et al., 2018; Thoms et al., 2015). In addition to its role in snoRNA release, Rok1 has also been shown to enable the release of Rrp5 to permit 40S maturation (Khoshnevis et al., 2016). Meanwhile, Has1 drives remodeling of domain I of $25 \mathrm{~S}$ to promote stable association of AFs and r-proteins including uL22, uL24, uL29 and eL37 (Brüning et al., 2018; Dembowski et al., 2013a). This proposed function correlates well with the identified binding site of Has 1 and recent structural data showing Has1 position on pre-ribosomes (Brüning et al., 2018; Kater et al., 2017; Sanghai et al., 2018). It has also been recently shown that the remodeling activity of DExD box protein Mak5 is required for the incorporation of r-protein uL16 in later stage of the assembly (Brüning et al., 2018). Moreover, the binding of export adaptor protein Arx1 requires structural rearrangement mediated by DExD box helicase Spb4 (Brüning et al., 2018). 
Putative remodeling function of other RNA helicase have also been described but the mechanistic details often remain enigmatic. For example, the DExD Dbp10 promotes 27SB pre-rRNA processing and is proposed to exert a remodeling activity necessary for PTC formation (Burger et al., 2000; Manikas et al., 2016). It has also been shown that deletion of the non-essential helicase Dbp3 results in a pre-rRNA processing defect similar to lack of RNase MRP, suggesting that Dbp3 might have a remodeling function to facilitate the recruitment of RNase MRP necessary for A3 cleavage (Kressler et al., 1999; Weaver et al., 1997). Analysis of pre-rRNA processing upon depletion of another non-essential helicase Dbp7 suggests its putative role, and possibly together with Dbp6 and Dbp9, in early stages of LSU biogenesis (Daugeron et al., 2001; Daugeron \& Linder, 1998; Kressler et al., 1998). Dbp6 is part of a large subcomplex consisting of Urb1/Npa1, Urb2/Npa2, Nop8, and Rsa3, which together has been recently reported to chaperone early folding events of LSU pre-rRNA (de la Cruz et al., 2004; Joret et al., 2018; Kressler et al., 1998; Rosado et al., 2007a; Rosado \& Cruz, 2004). Genetic interactions were observed between the r-protein uL3 and members of Dbp6-containing complex as well as Dbp7 and Dbp9, suggesting that their functions may be linked to the incorporation of r-protein uL3 into pre-60S particle (Rosado et al., 2007a). However, the precise function of each these three helicases, the interplay among them, and the molecular mechanisms that lead to the recruitment of uL3 remains to be elucidated. 


\section{Chapter Two: Scope and aim of the study}

Ribosome biogenesis involves the processing, modification and folding of precursor ribosomal RNAs as well as the correct assembly of r-proteins. These processes are coupled with structural transitions that are highly orchestrated and with the transient association and dissociation of approximately 200 assembly factors (AF). A number of these AF are RNA helicases, which through their energy-dependent function can remodel RNAs and RNA-protein complexes. In S. cerevisiae, 21 RNA helicases have been implicated in ribosomal subunit assembly. While some have been functionally characterized, the precise role of the other RNA helicases remains lacking. Therefore, this work aimed to expand the knowledge on helicase functions in ribosome assembly and provide insights into the molecular roles of two nonessential DExD box RNA helicases Dbp3 and Dbp7 in the course of large subunit biogenesis.

To achieve the aim of the study, the following specific objectives were addressed:

- To demonstrate the catalytic activity of Dbp7 and Dbp3 and their mutants in vitro

- To determine whether their catalytic activity is required for their functions in ribosome biogenesis

- To identify the binding site of these candidate RNA helicases on their RNA substrates in vivo

- To explore their function in regulation pre-rRNA processing, rRNA modification and composition of pre-ribosomal particles

- To investigate how their remodeling activities contribute to the early pre-rRNA remodeling events during large subunit biogenesis 


\section{Chapter Three: Manuscript I}

Aquino GRR, Hackert P, Krogh N, Pan KT, Nielsen H, Urlaub H, Bohnsack KE, Bohnsack MT. The RNA helicase Dbp7 promotes domain V/VI compaction and stabilization of inter-domain interactions during early $60 \mathrm{~S}$ assembly. Nat. Commun. Under review.

\section{Authors contributions}

Figure 1: Generation of yeast strains and complementation system (GRRA); Analysis of prerRNA processing (GRRA); Molecular cloning and protein expression and purification (GRRA); NADH-coupled ATPase assay (GRRA); Spot test assay for analysis of growth (GRRA)

Figure 2: Sucrose density gradient analysis (GRRA); Immunoprecipitation for analysis of prerRNA and protein composition (GRRA); Mass spectrometry (HU, KTP); Confirmatory western blot analysis (GRRA)

Figure 3: CRAC experiment (PH); Bioinformatic analysis of CRAC data (see below)

Figure 4: Bioinformatic analysis of CRAC data (see below); Pre-ribosomal distribution of snoRNAs using sucrose density gradient centrifugation (GRRA); Analysis of snoRNAs association in Dbp7- and Nop2-containing pre-ribosomal particle (GRRA)

Figure 5: Pulldown of Nop2-containing particles (GRRA); Mass Spectrometry (HU, KTP); Confirmatory western blot analysis (GRRA); Generation of yeast strains and complementation system and western blot analysis of uL3 recruitment (GRRA)

Figure S1: Generation of yeast strains, depletion assay, growth assay, analysis of protein expression level, analysis of pre-rRNA processing and quantification, and cellular localization using immunofluorescence microscopy (GRRA)

Figure S2: Preparation of RNA samples for analysis (GRRA); RiboMeth seq experiment (NK); Mapping of data in 3D structure (GRRA)

Figure S3: Pulldown of Nop2- and Npa2-contaning particles followed by western blot and northern blot analysis (GRRA)

Figure S4: Dimethyl sulfide probing experiment (GRRA)

Non-experimental contribution: MTB and KEB conceived the study and supervised the work. HU, KTP, GRRA, KEB and MTB analyzed mass spectrometry data. HN, NK, GRRA, KEB and MTB analyzed RiboMeth-seq data. Jens Kretschmer and Lukas Brüning contributed to bioinformatics analysis of CRAC data also performed by GRRA, KEB and MTB. GRRA wrote the first draft of the manuscript and prepared the figures. This draft version of the manuscript was revised with the support of KEB and MTB. 


\section{The RNA helicase Dbp7 promotes domain V/VI compaction and stabilization of inter-domain interactions during early 605 assembly}

Gerald Ryan R. Aquino ${ }^{1}$, Philipp Hackert ${ }^{1}$, Nicolai Krogh², Kuan-Ting Pan ${ }^{3 \S}$, Henrik Nielsen², Henning Urlaub ${ }^{3}$, Katherine E. Bohnsack ${ }^{1 \#}$ and Markus T. Bohnsack ${ }^{1,4 \#}$

${ }^{1}$ Department of Molecular Biology, University Medical Center Göttingen, Humboldtallee 23, 37073 Göttingen, Germany

2 Department of Cellular and Molecular Medicine, University of Copenhagen, 2200N Copenhagen, Denmark

${ }^{3}$ Max Planck Institute for Biophysical Chemistry, Bioanalytical Mass Spectrometry, 37077 Göttingen, Germany

${ }^{4}$ Göttingen Centre for Molecular Biosciences, Georg-August-University, Justus-von-Liebig Weg 11, 37073 Göttingen, Germany

\# Correspondence should be addressed to:

Markus T. Bohnsack (Email: markus.bohnsack@med.uni-goettingen.de)

Katherine E. Bohnsack (Email: katherine.bohnsack@med.uni-goettingen.de)

$\S$ Present address: Hematology/Oncology, Department of Medicine II, Johann Wolfgang Goethe University, 60590, Frankfurt am Main, Germany; Frankfurt Cancer Institute, Goethe University, 60596, Frankfurt am Main, Germany

Running title: $\mathrm{Dbp} 7$ in early $60 \mathrm{~S}$ biogenesis

Keywords: RNA helicase, ribosome biogenesis, small nucleolar RNA, ribosomal protein. 


\section{ABSTRACT}

A key early event in ribosome biogenesis involves the folding of precursor ribosomal RNA (prerRNA) concurrent with the association of ribosomal proteins and assembly factors (AFs) to establish the different structural domains and their subsequent clustering. Several RNA helicases have been implicated in this compaction event through their energy-dependent remodeling functions. Here, we demonstrate how the remodeling activity of the DEAD-box RNA helicase Dbp7 contributes to the formation of inter-domain interactions. Characterization of Dbp7-containing particles showed evident association of r-protein uL3 and snR190, which both interacts to several pre-rRNA domains, as well as AFs that serve as scaffolds for prerRNA folding. Compositional analysis of a later Nop2-contaning pre-60S particle revealed the retention of snR190 and diminished incorporation of uL3 when Dbp7 is lacking. This suggests that Dbp7's remodeling activity is linked to the release of snR190 and recruitment of uL3, which we here propose to occur sequentially. Using in vivo crosslinking, we identify Dbp7's binding site on domain V and VI of pre-rRNA strategic for this function. Moreover, its catalytic activity is required for these remodeling events. Altogether, our findings expand the reported molecular functions of RNA helicases and provide new insights into the very early events of large subunit biogenesis. 
Ribosomes are highly conserved molecular machines that decode the messenger RNA (mRNA) to produce cellular proteins. This key function relies on their highly conserved twosubunit structure composed of ribosomal proteins ( $r$-proteins) and four ribosomal RNAs (rRNA). In the yeast Saccharomyces cerevisiae, ribosome biogenesis begins with nucleolar transcription to generate a single, large $35 \mathrm{~S}$ precursor rRNA (pre-rRNA) containing the sequences of the mature $18 \mathrm{~S}, 5.8 \mathrm{~S}$ and 25 rRNAs. As the transcript emerges, it associates with a subset of ribosomal proteins and numerous assembly factors (AFs) to form the SSU processeome and $90 \mathrm{~S}$ pre-ribosomal complexes ${ }^{1}$. Concomitantly, the pre-rRNA undergoes exo- and endonucleolytic processing (Figure 1A) to remove the internal and external transcribed spacers (ITS and ETS) ${ }^{2}$. One key endonucleolytic cleavage event (A2) generates the earliest pre-40S and pre-60S particle, both of which then proceed independent biogenesis pathway ${ }^{3}$. As part of its maturation, the rRNA also undergoes numerous modification installed by small nucleolar RNPS (snoRNPS), which contains small nucleolar RNAs (snoRNAs) that base pairs with the pre-rRNA to guide 2'-O-methylation, pseudouridylation, acetylation, in addition to substrate-specific enzymes ${ }^{4,5}$. Aside from rRNA modification, recent findings proposed that some snoRNPs can function as rRNA chaperone to assist in the pre-rRNA folding process ${ }^{4,6-10}$.

The early stages of large subunit assembly likely include events that bring together and stabilize the different RNP domains of the pre-60S particle. In its final architecture, the 60S subunit has six defined structural domains (I to VI from 5' to 3') connected to each other by the clustering of root helices that precede each domain ${ }^{11}$. It believed that the earliest assembly involves the association of early r-proteins and AFs to the solvent-exposed structural domains I and II leading to the folding and stabilization of these domains followed by those of domain $\mathrm{VI}^{12-14}$. This results to the formation and subsequent clustering of the respective root helices, which in turn are required for early processing of the 25S rRNA (27SA to 27SB) as well as later steps of maturation. These insights are further supported by recent cryo-EM structures of yeast nucleolar pre-60S particles ${ }^{15-17}$. Several studies have described how large AFs such as Rrp5 and Npa1 can act as rigid scaffold and binding platforms for other AFs to collectively mediate initial compaction and stabilization ${ }^{8,18-20}$. Additionally, the early binding r-protein uL3 is also deemed crucial for the stabilization of earliest 605 intermediates and incorporation of all other $60 S$ ribosomal protein ${ }^{12,14,21-24}$. To date, the mechanistic details of how AFs and r-proteins mediate early compaction as well as the interplay among them are lacking.

RNA helicases are ubiquitously expressed proteins that use the energy of NTP-binding and hydrolysis to remodel RNA and RNP complexes and therefore can promote structural transitions during the ribosome assembly process. Out of the approximately $30 \mathrm{AFs}$ that transiently associates with the earliest pre-60S particle, eight of those are RNA helicases (Dbp3, Dbp6, Dbp7, Dbp9, Has1, Mak5, Prp43, Drs1) ${ }^{25}$. Among these early-acting RNA 
helicases, the role of Prp43, Has1, Dbp3 in promoting the release of specific snoRNAs have been reported ${ }^{26,27}$. In addition, Mak5 and Has 1 have been proposed to facilitate the recruitment of particular r-proteins and AFs, respectively ${ }^{27,28}$. The functions of other RNA helicases namely Dbp6, Dbp7 and Dbp9 remain poorly characterized to date. Genetic interactions have been reported among these three RNA helicases and with the components of Npa1 complex (Npa1, Npa2, Nop8, and Rsa3) as well as uL3, suggesting that they may function cooperatively ${ }^{24,29-}$ 32. Recently, evidences have been provided suggesting that Dbp6 functions with the Npa1 complex to facilitate early clustering of root helices ${ }^{8}$. However, the possible roles of the two other DExD-box RNA helicases Dbp7 and Dbp9 in early pre-60S compaction and their functional link with uL3 remains unknown.

In this work, we provide key evidences supporting Dbp7 function in the very early stages of large subunit assembly. We confirm that $\mathrm{Dbp} 7$ is present in nascent pre-60S particles together with uL3 and the Npa1 complex. We show using in vivo photoactivatable ribonucleosideenhanced crosslinking and analysis of $\underline{\underline{c}} \mathrm{DNA}$ (PAR-CRAC) that Dbp7 binds at the domain V/VI of 25s rRNA close to the binding site of UL3 in early nucleolar pre-60S ribosome. Consistent with these findings, the absence of Dbp7 results to inefficient recruitment of uL3 and to accumulation of snoRNAs with targets close to the Dbp7 binding site including the chaperone snR190. These data suggest a remodeling function of Dbp7 resulting to the release of snR190 concomitant with the exit of Npa1 complex from early pre-60S particle, thereby facilitate the incorporation of uL3 to further stabilize the domain I and VI interaction. Moreover, the catalytic activity of Dbp7 is essential in this remodeling activity. Our findings expand the existing knowledge on how the early remodeling events are coordinated by the early AFs to facilitate the initial compaction of the nascent 605 . 


\section{RESULTS}

The ATPase activity of $\mathrm{Dbp} 7$ is required for efficient pre-rRNA processing and rRNA methylation

The DExD box protein Dbp7 is a non-essential RNA helicase implicated in large subunit biogenesis ${ }^{30}$ but its function(s) during the assembly process remains poorly characterized. To verify its role in the early 60S biogenesis, we examined pre-rRNA processing (Figure 1A) in the absence of $\mathrm{Dbp} 7$ using $\Delta d b p 7$ strain. Northern blot analyses revealed that compared to wild-type, $\Delta d b p 7$ strain showed significant accumulation of the $35 S$ pre-rRNA and a mild increase in 35S/33S pre-rRNA intermediates (Figure 1B; Supplementary S1A). Concomitantly, the steady-state level of 27SA pre-rRNA was slightly reduced, whereas a significant decrease in 27SB was observed. These observations suggest that $\mathrm{Dpb} 7$ is required for efficient cleavage at the $A_{2}$ site and conversion of the 27SA pre-rRNAs into 27SB via processing at the $A_{3}$ and $B_{1 L / S}$ sites (Figure 1A) and this corroborate well with its observed localization in the nucleolus (Supplementary Figure S1B). Additionally, growth analysis revealed that $\Delta d b p 7$ strain has a doubling rate of twice slower than the wild-type (Supplementary Figure S1C). To confirm that the observed effects are caused by lack of Dbp7 and not secondary effects arising in deletion strain, we established a system for transient depletion of Dbp7 in which the expression of $D B P 7$ is under the control of a truncated galactose-inducible, glucose-repressible promoter $\left(p G a l_{s}\right)$. The expression level of 3HA-Dbp7 from this promoter is higher compared to the endogenous protein level (Supplementary Figure S1D). However, the pre-rRNA processing was comparable to that in the wild-type (Supplementary Figure S1E). 3HA-Dbp7 was undetectable after $10 \mathrm{~h}$ of growth in glucosecontaining media but the pGALs-3HA-Dbp7 strain displayed normal growth until $12 \mathrm{~h}$ growth (Supplementary Figure S1F-G). Importantly, consistent with the pre-rRNA processing defects observed in $\Delta d b p 7$ strain, depletion of Dbp7 leads to accumulation of the 35S and 32/33S pre-rRNAs as well as reduced level of the 27SB pre-rRNA (Figure1B; Supplementary Figure S1E and S1H). However, in contrast to the $\Delta d b p 7$ strain where a mild decrease in the 27SA and 20S intermediates was observed, transient depletion of Dbp7 did not affect the 20S pre-rRNA level and 27SA was slightly accumulated (Figure1B; Supplementary Figure S1E and $\mathbf{S 1 H}$ ). Collectively, our results demonstrate that Dbp7 is important for early steps in prerRNA processing and normal growth.

As a putative RNA-dependent ATPase and RNA helicase, we next wanted to investigate whether the Dbp7 possess such catalytic activity and if this is required for its function in ribosome biogenesis. To address this, His ${ }_{10}$-ZZ-tagged wild-type or mutant version of Dbp7 were first recombinantly expressed in E. coli and purified (Figure 1C). The mutant Dbp7 (Dbp7DQGD) was generated by glutamate $(E)$ to glutamine $(\mathrm{Q})$ substitution in the conserved sequence motif DEGD. The purified proteins were then used for in vitro NADH-coupled 
ATPase assays in the presence or absence of RNA substrate. Mutation of the DEGD sequence motif drastically decreased the ATPase activity of Dbp7 in vitro similar to background (Figure 1D). Furthermore, there is a significant increase in ATPase activity of wild-type Dbp7 in the presence of RNA, demonstrating that Dbp7 is indeed an RNA-dependent ATPase.

Having confirmed that $E$ to $Q$ substitution within the DEGD sequence motif renders Dbp7 catalytically inactive in vitro, a yeast complementation system was then generated to assess the requirement for the catalytic activity of Dbp7 in vivo. In this complementation system, the $\Delta d b p 7$ strain was transformed with a low-copy number plasmid containing the coding sequence (CDS) of wild-type or catalytically inactive Dbp7 including their endogenous promoter, or an empty plasmid as a control. The resulting strains were first subjected to spot test assay and this revealed that the growth defect caused by the absence of endogenous Dbp7 can be rescued by plasmid-derived Dbp7 (Figure 1E). Expression of Dbp7DQGD resulted to a more pronounced growth retardation than lack of Dbp7 ( $\Delta d b p 7+E V)$ (Figure 1E), implying that, similar to other large subunit RNA helicases ${ }^{27}$, substitutions within this common sequence motif can have a dominant negative effect on cell growth. Pre-rRNA processing was then analyzed using these strains. Consistent with our earlier findings, the $\Delta d b p 7$ strain carrying an empty vector showed accumulation of earlier precursors (35S, 33S/32S and 27SA) and marked decrease in the 27SB level compared to wild-type yeast also carrying the empty vector (Figure 1F; Supplementary Figure S1I). These pre-rRNA processing defects are largely rescued by plasmid-derived expression of a wild-type Dbp7. Expression of catalytically inactive Dbp7 ${ }_{D Q G D}$ resulted to a pre-rRNA processing defects similar to that of cells lacking Dbp7 (Figure 1F; Supplementary Figure S1I), indicating that the ATPase activity of Dbp7 is required for normal pre-rRNA processing.

Alongside pre-rRNA processing, installation of modifications, such as of 2'-O-methylations, constitutes one of the rRNA maturation steps and early events in ribosome biogenesis ${ }^{33}$. To explore a putative role of $\mathrm{Dbp} 7$ and its catalytic activity in regulating 2'-O-methylation, we employed a high-throughput approach using RiboMeth-seq (RMS) ${ }^{34,35}$ to monitor the extent of 2'-O-methylation in all sites in the rRNAs using the established Dbp7 complementation system. Our results reveal only mild but significant changes in the level of 2'-O-methylation of some nucleotides within the 25S rRNA (C663, U898, C2197, U234, A2640, and C2948), while methylation of the 18S rRNA was unaffected (Supplementary Figure S2A-B). In all cases, except C2948, the RMS score is lower in the absence of Dbp7 compared to wild-type. The mild defects in methylation were rescued by expression of Dbp7WT from a plasmid but the expression of Dbp7DQGD showed lower methylation similar to when Dbp7 is lacking. Mapping these sites in an early pre-60S particle (PDB: $3 \mathrm{JCT})^{36}$ did not reveal apparent spatial clustering of these modifications and therefore does not support direct regulation of methylation by Dbp7 
bound to specific pre-60S region (Supplementary Figure S2C). This rather suggests that the effects on 25S rRNA 2'-O-methylation could be indirect consequence of Dbp7 function.

\section{Dbp7 associates with early pre-60S particles}

To provide further evidence on the function of Dbp7 in large subunit synthesis, we first sought to determine whether $\mathrm{Dbp} 7$ associates with pre-60S ribosomal particles by sucrose density gradient centrifugation. Separated gradient fractions of whole cell lysate from a yeast strain expressing Dbp7-TAP were subjected to western blotting to check the distribution of Dbp7 among pre-ribosomal complexes. Our result shows enrichment of Dbp7-TAP in factions containing (pre-)60S ribosomal complexes but also co-migration with (pre-)40S subunits (Figure 2A).

Next, we want to establish whether Dbp7 associates with pre-rRNA(s) found in pre-60S ribosomal particles. For this, Dbp7-containing particles were isolated from whole cell lysates via TAP-tag and the co-purified pre-rRNAs were extracted and determined by northern blot analysis. Extracts from wild-type yeast were analyzed in parallel to control for specific enrichment. Detection using probes that hybridize to the ITS1 and ITS2 region revealed strong association of 27SA pre-rRNA species as well as weaker enrichment of the $35 \mathrm{~S}$ and 33S/32S intermediates (Figure 2B). This, therefore, confirms the association of Dbp7 with pre-60S particles. Moreover, this is consistent with the observed pre-rRNA processing defect when $\mathrm{Dbp} 7$ is lacking and it is likely that $\mathrm{Dbp} 7$ contributes in the processing and/or stability of $27 \mathrm{SA}$ pre-rRNA.

To gain further insights into the pre-ribosomal particles Dbp7 associates with, isolated Dbp7TAP particles were analyzed for protein composition using mass spectrometry (Figure 2C-D). Our result identified AFs (e.g. Brx1, Rrp5, Nop4, Nop8, Nop2) that associate with very early, nucleolar pre-60S particles (Figure 2D; Supplementary Table S1) consistent with the presence of 27SA pre-rRNA in Dbp7-containing pre-60S particle. Additionally, early-binding large subunit ribosomal proteins (e.g. uL3, uL4, eL19, uL23, eL15) are present. Notably, the most enriched protein was uL3 supporting an earlier report of its genetic network of interaction including $\mathrm{Dbp} 7^{32}$. The association of uL3 in Dbp7-TAP particle was verified in an independent western blot analysis (Figure 2E). Additionally, we also identified the association of Dbp7 with two other RNA helicases, Dbp6 and Dbp9. Along this line, our data also identified components of the Npa1 complex (Npa1, Npa2, Nop8, and Rsa3) reported to mediate the initial compaction of earliest large ribosomal subunit precursor in coordination with the RNA helicase Dbp6 ${ }^{8}$. Furthermore, we have confirmed in a reverse pulldown followed by western blotting the presence of Dbp7 in Npa2-containing particle (Supplementary Figure S3A). The network of genetic interaction described among these proteins may arise from physical interactions and together, provide a compelling evidence for the functional role of $\mathrm{Dbp} 7$ with these proteins in 
large subunit biogenesis. Interestingly, there is no notable enrichment of small subunit AFs despite detection of Dbp7 in (pre)-40S complexes (Figure 2A; Supplementary Table 1). Furthermore, components of the Box $C / D$ and H/ACA snoRNPs were also identified. This indicates that Dbp7 is recruited to the pre-60S particles while rRNA modifications are being installed and/or while snoRNPs chaperoning pre-rRNA folding are present. Likewise, this suggests that $\mathrm{Dbp} 7$ may regulate the interaction of these snoRNPs with the pre-ribosome as partially supported by our RMS data. Collectively, our results reveal that Dbp7 associates with very early pre-60S particles and suggest potential roles of Dbp7 in regulating snoRNAs dynamics on pre-ribosome and/or mediating initial compaction of the nascent 605 together with the Npa1 complex.

\section{Dbp7 crosslinks to specific sites within domain V and VI of the 25S rRNA}

One key information in elucidating the function of RNA helicases within large RNPs is their binding site. To identify the RNA sequences bound by Dbp7 in cell, we employ the in vivo photoactivatable ribonucleoside-enhanced crosslinking and analysis of $\underline{c} D N A$ (PAR-CRAC) approach ${ }^{37-39}$. Wild-type yeast and a yeast strain expressing C-terminally His-TEV protease site-Protein A (HTP)-tagged Dbp7 were first grown in medium containing 4-thiouracul (4sU) prior to crosslinking using light at $365 \mathrm{~nm}$. Dbp7-RNA complexes were purified under native and denaturing conditions. A cDNA library is then generated from the partially digested, bound RNA for Illumina deep sequencing. The sequencing data obtained were analyzed using an inhouse pipeline to filter reads containing T-to-C misincorporations introduced during reverse transcription of RNAs containing 4sU followed by mapping onto Saccharomyces cerevisiae genome. Two CRAC experiments were done to confirm the reproducibility our results and, in both cases, analysis of distribution of reads mapping to different classes of RNAs revealed a significantly larger portion of reads (67-69\%) corresponding to ribosomal RNAs (rRNAs) in Dbp7-HTP expressing cells compared to the wild-type (31-36\%) (Figure 3A). The fragments present in the wild-type control sample reflect the non-specific, background RNAs that are carried over during the CRAC procedure. Nevertheless, the substantial enrichment of reads mapping to rRNAs in the Dbp7-HTP expressing cells further underlines the involvement of Dbp7 in ribosome biogenesis.

Given the high reproducibility of the PAR-CRAC results, a representative dataset is presented in the subsequent analysis. Mapping of the normalized sequencing reads to each nucleotide of the RDNA locus revealed three distinct peaks (peaks 1-3) in the 25S rRNA close to the 3' end representing Dbp7 crosslinking sites (Figure 3B; upper panel). These three peaks do not overlap with background peaks from the wild-type control sample and are also specific when compared to the crosslinking sites of other RNA helicases we have also previously identified $^{26,27,40}$. Furthermore, the binding of $\mathrm{Dbp} 7$ in this region is confirmed by the detectable 
presence of T-to-C misincorporations introduced to the nucleotide upon crosslinking to amino acids followed by reverse transcription (Figure 3B; lower panel). Two-dimensional arrangement of these three distinct peaks was then examined by modelling them into the secondary structure of mature $25 \mathrm{~S}$ rRNA ${ }^{41}$. We found out that the Dbp7-HTP crosslinking sites lie in in helix 90 of domain V and in helices 94, 98 and 99 in the domain VI (Figure 3C). These crosslinking sites correlates well with the function of $\mathrm{Dbp} 7$ in the early events of $60 \mathrm{~S}$ biogenesis as this domain $\mathrm{VI}$, together with domains I and II, are the regions first to show folding and stabilization ${ }^{12,13,15,16}$ and helix 94 is a domain VI root helix. Interestingly, both strands of helices 98 and 99, which are separate by a 100-nucleotide loop sequence, are crosslinked by Dbp7HTP. This suggests that the basepairing of helices 98 and 99 observed in mature ribosomes is already established during the initial steps of pre-60S biogenesis (Figure $3 \mathrm{C}$ ). To obtain a structural context of this newly identified crosslinking sites, we then mapped our CRAC data onto the tertiary structure of pre-60S particles. To date, Dbp7 is not present any available cryoEM structures of pre-60S particles ${ }^{15-17,36,42-46}$. As our data suggests a role of Dbp7 in the early assembly steps, the identified crosslinking sites were then mapped onto the recently resolved cryo-EM structures of five nucleolar pre-60S intermediates ${ }^{15}$. Among the different nucleolar pre-60s structures, we can only completely map the three crosslinking sites in the so-called state C (PDB:6EM1) as the earliest particle possible. The three Dbp7-HTP crosslinking sites cluster in close proximity, supporting the notion that they reflect different contact points of a single Dbp7 binding event (Figure 3D). Furthermore, Examination of nearby r-proteins revealed $\mathrm{UL} 3$ in close proximity to the Dbp7 binding site consistent with strong enrichment of this ribosomal protein in Dbp7-containing pre-60S particles (Figure 2C-D; Figure 3D). Overall, our CRAC data reveal a binding site that is coherent with the timing of function and particle composition of Dbp7.

\section{A subset of snoRNAs with pre-rRNA target site proximal to Dbp7 crosslinking sites accumulates on pre-60S particles when Dbp7 is lacking}

As the identified Dbp7 crosslinking site on the 25S rRNA is in close proximity to the pre-rRNA target sites of several snoRNAs (Figure $3 \mathrm{C}$ ) and snoRNP proteins were co-purified with Dbp7TAP (Figure 2D), the identities of the snoRNAs enriched in the Dbp7-HTP PAR-CRAC data were investigated. This revealed a more than two-fold enrichment of several snoRNAs (snR190 (H/ACA), snR49 (H/ACA), snR73 (C/D), snR34 (H/ACA), snR82 (H/ACA) and snR42 $(H / A C A)$ ) relative to the wild-type control (Figure 4A; Supplementary Table S2). To substantiate this initial finding, we performed a TAP-tag pulldown of Dbp7 and checked the presence of these enriched snoRNAs by northern blotting. snR190 and snR73, snR82 and snR42 were strongly enriched in Dbp7-containing particles, whereas snR34 and snR49 were detected slightly above background levels (Figure 4B). The lesser association of snR34 and 
snR49 can be attributed to the weak or transient association of these snoRNAs and may only be captured by crosslinking. The specific enrichment of a subset of snoRNAs is supported by the finding that snR63, which was not enriched in the Dbp7-HTP PAR-CRAC data, was not enriched above background in the Dbp7-TAP pulldown.

Next, we asked if the lack of Dbp7 affects the dynamics of these snoRNAs on pre-ribosomes. To investigate this, whole cell lysate of wild-type and $\Delta d b p 7$ strains were fractionated by sucrose density centrifugation. Absorption profile at $260 \mathrm{~nm}$ revealed defects in large subunit biogenesis characterized by strong reduction in $60 \mathrm{~S}$ relative to $40 \mathrm{~S}$ and a concomitant decrease in 80 monosomes (Figure 4C; upper panel). Furthermore, the amount of eL15 comigrating with large pre-60S ribosomal complexes are markedly reduced indicating impairment in early $60 \mathrm{~S}$ biogenesis. Northern blot analysis of the fractionated lysates revealed the distribution profile between ribosomal and non-ribosomal fractions to be comparable between the WT and $\Delta d b p 7$ strains for snR49, snR73, snR82, snR42 and snR63 (Figure 4C). Interestingly, while a similar amount of snR190 in fractions containing non-ribosomal (fractions 1-7) and large pre-ribosomal complexes (fractions 12-15) can be observed in the WT cells, more snR190 is present in the pre-60S fractions than non-ribosomal fractions of $\Delta d b p 7$ cells suggesting accumulation of snR190 in the pre-ribosomal complexes. Moreover, our results also show a notable increase of snR34 in the large pre-ribosomal fractions as well as in later fractions (fractions 18-20) of cells lacking Dbp7 compared to wild-type.

To investigate a putative role of Dbp7 in modulating snoRNA levels on pre-60S complexes, we then sought to monitor the levels of these subset of snoRNAs in Nop2-containing pre-60s particles of wild-type yeast and the strain lacking Dbp7. Nop2 is present in the so-called state D/E cryo-EM structure of nucleolar 605 precursor and positioned far away from the identified Dbp7 binding site here ${ }^{15}$. Nop2 was identified in purified Dbp7-TAP particles (Figure 2C-D) and conversely, Dbp7 is present in Nop2-containing pre-60S particles as revealed by a reverse western blot analysis (Supplementary Figure S3A). Moreover, analysis of pre-rRNAs present in Nop2-associated pre-60S particle revealed strong enrichment of 27SB pre-rRNA (Supplementary Figure S3B), implying that although Dbp7 and Nop2 are both present in common pre-60S complexes, Nop2 remains associated after dissociation of Dbp7. Collectively, these observations support a strategic approach to use Nop2-containing particles to investigate changes in the maturation pathway when Dbp7 is lacking. Pre-60S complexes were then isolated via Nop2-TAP from WT cells and those lacking Dbp7 and the levels of selected snoRNAs were subsequently monitored by northern blotting. Our results reveal strong accumulation of snR190, snR34, snR73, snR82 and snR42 in Nop2-containing particle of cells lacking Dbp7 compared to wild-type, while the levels of snR49 and snR63 were unaffected (Figure 4D). Interestingly, most of these snoRNAs belong to the H/ACA box family except for snR73 and snR190. This partially explains the mild effects observed in our RMS data when 
Dbp7 is absent. More importantly, except for snR49 and snR63, these snoRNAs guide modifications close to the identified Dbp7 crosslinking sites (Figure $3 \mathbf{C}$ ). In the case of snR34, despite its observed weak association in Dbp7-containing particle (Figure 4B), its strong retention in Nop2-containing particle when Dbp7 is lacking strongly suggests that Dbp7 affects its dynamics in pre-60S particle. This can be explained by its pre-rRNA target site located within the identified Dbp7 binding site in helix 90, which further supports our CRAC data (Figure $3 C$ ). Therefore, it is possible therefore that recruitment and/or remodeling activity of $\mathrm{Dbp} 7$ is coupled to the release of these snoRNAs from their basepairing sites in domain V/VI. snR190 has a predicted 2'-O-methylation site at G2395 close to the Dbp7 pre-rRNA crosslinking sites (Figure $\mathbf{3 C}$ ); however, methylation at this site has not been detected ${ }^{34,47}$. Recently, another predicted basepairing site located in domain I of the 25S rRNA has been reported, and together with the known basepairing site in V, snR190 has been proposed to function as pre-rRNA folding chaperone (Figure $4 E$, box $A$ and $B$ in bottom panel) ${ }^{8}$. As snR190 has the highest enrichment among the snoRNAs in our Dbp7-HTP PAR-CRAC data, we then examined whether Dbp7 physically associates with the regions of snR190 involved in the basepairing with the $25 \mathrm{~S}$ rRNA. Analysis of distribution reads revealed majority of them mapping across the box B sequence that interacts with domain I, close to the 3 ' end of snoRNA (Figure 4E). This is further verified by the presence of T-to-C misincorporations. Reexamination of the crosslinking profile of Dbp7-HTP on pre-rRNA revealed a small enrichment of sequencing reads on the predicted snR190 baseparing site on domain I (peak 4) (Figure 3B). Interestingly, our mutation profile is distinct from a previously reported PAR-CRAC data of Npa1, which directly binds to the snR190 pre-rRNA guide sequence ${ }^{8}$. It is therefore tempting to speculate that $\mathrm{Dbp} 7$ transiently binds to the box B region snR190 and domain I of $25 \mathrm{~S}$ when they are basepaired. Furthermore, the close proximity of the predicted snR190 baseparing site in domain $\mathrm{V}$ to the $\mathrm{Dbp} 7$ pre-rRNA crosslinking site (Figure 3D) may partly explain why lack of Dbp7 leads to strong accumulation of snR190. Altogether, our data suggest that in maturing nucleolar pre-60s particles the lack of Dbp7 compromises remodeling events necessary for the release of snoRNAs with pre-rRNA target sites close to Dbp7 binding region.

\section{Dbp7 is required for the efficient recruitment of uL3 to pre-60S particles to stabilize the domain I-domain VI interaction}

The retention of snoRNAs, including snR190, in pre-60S complexes and the association of earlier rRNA precursors (27SA, 33S, 35S) in the Nop2-containing particle of $\Delta d b p 7$ cells (Supplementary Figure S3B), indicates perturbations in the early stages of ribosome assembly. This likely arises from inefficient incorporation of $r$-proteins and/or failure to recruit or release AFs when Dbp7 is lacking. This hypothesis is supported by the proposed role of 
snR190 in mediating interaction of domains I and $V^{8}$, which is a key early maturation event that is likely important for downstream maturation steps such as recruitment of RP and/or AFs.

To assess the effect on protein composition of pre-60S complexes when Dbp7 is lacking, Nop2-containing particles were isolated from whole cell lysate of WT and $\Delta d b p 7$ cells and protein composition was analyzed using mass spectrometry. Analysis of the obtained MS data showed a decrease in many proteins in the Nop2-containing pre-60S particle in the absence of Dbp7 (Figure5A-B; Supplementary Table S3). Among the AFs, a notable decrease in "A3 factors" (e.g. Rlp7, Cic1, Nop15, Nop7) was observed. This group of AFs are key players in the processing of $27 \mathrm{SA}$ to $27 \mathrm{SB}^{48}$ and therefore corroborates the pre-rRNA processing defects observed in the absence of Dbp7 (Figure 1B and 1F; Supplementary Figure S3B). The observed decrease of these "A3 factors" is further confirmed by an independent western blot analysis of Cic1 (Figure 5C). Furthermore, dimethylsulfide (DMS) structure probing revealed changes in nucleotide accessibility of ITS2 region bound by these factors in $\Delta d b p 7$ cells compared to wild-type cells (Supplementary Figure S4), supporting the impaired recruitment of these AFs. Consistent with the hierarchical pathway of stable association of AF in early $60 \mathrm{~S}$ particles ${ }^{48}$, we also noted a decrease in B-factors (Figure 5B, Supplementary Table S3). Given these observations, we anticipate that the remodeling activity of $\mathrm{Dbp} 7$ contributes to the recruitment and/or stable integration of the A3 factors to pre-60S particles.

In contrast, we observed an increase in AFs (e.g. Npa1, Npa2, Nop8, Rsa3, Dbp6, Dbp9, Dbp3, Mak5, Rrp5, Nop4, Nop12, Prp43, Cbf5) that participate in the earliest co-transcriptional stages of $60 \mathrm{~S}$ biogenesis ${ }^{13,48}$ and are associated with Dbp7-contaning particle (Figure 2B; Supplementary Table S1). The retention of this AFs in a later Nop2-containing particle suggests a blockage of maturation and/or incorrect assembly of a very early pre-60S. This is consistent with the detection of earlier 35S and 27SA pre-rRNAs in Nop2-contaning particle in the absence of Dbp7 (Supplementary Figure S3B). The retention of the Npa1 complex (Npa1, Npa2, Nop8, Rsa3, and Dbp6) and the observed accumulation of Npa1-bound snR190 in pre-60S particles when Dbp7 is lacking support a model that Dbp7 may, directly or indirectly, release these complex from pre-60S complexes. Furthermore, the enrichment of Cbf5 and Gar1 corroborates the observed retention of several H/ACA snoRNAs in Nop2-containing particle when Dbp7 is lacking (Figure 4D; Figure 5B). Altogether, our data indicate that the pre-ribosomes purified from $\Delta d b p 7$ cells remains as very early assembly intermediates highlighting a key role of Dbp7 in driving efficient maturation of early pre-60S.

Examination of ribosomal proteins revealed a lesser range of effect to most early-binding large subunit ribosomal proteins (Figure5A-C, Supplementary Table S3). We found eL38, uL24, uL14, eL31, and eL21 to be most affected, i.e. more than 2-fold decrease. Most were not identified in Dbp7-contaning particle (Supplementary Table S1). Additionally, all of them were reported to cause significant accumulation of 27SB upon depletion except eL38 and eL21, the 
latter being required for $7 S$ processing ${ }^{12,21,23,49}$. Accordingly, most are present only in a later nucleolar particle ${ }^{15}$. Moreover, these r-proteins are non-essential ${ }^{21}$. Interestingly, the essential r-protein whose presence in the Nop2-TAP particle was most strongly affected by lack of Dbp7 was uL3. In contrast to the other affected r-protein, uL3 is present in earlier nucleolar particle state $\mathrm{C}^{15}$ and has been suggested to be crucial in the recruitment and stable incorporation of other large subunit ribosomal protein ${ }^{12,22}$. Hence, it is very likely that the significant decrease of eL38, uL24, uL14, eL31 and eL21 is due to inefficient incorporation of uL3 in the early pre60 s particle. Furthermore, the observed subtle decrease of a critical ribosomal protein reflects the non-essential nature of $\mathrm{Dbp} 7$ and also opens the possibility of overlapping or redundant functions with other related RNA helicase and AFs. The observed decrease in uL3 when Dbp7 is lacking, together with its strong association in Dbp7-containing particle and its position in the early pre-60s particle close to our identified Dbp7 binding site, provide compelling evidences for the functional link between uL3 and Dbp7 (see Discussion). The decrease in uL3 is further validated by independent western blot analysis of Nop2-containing particle in the presence or absence of Dbp7 (Figure $5 \mathrm{C}$ ). Our results indicate that Dbp7 is required for the recruitment and possibly stable integration of uL3 in earlier pre-60S particle. Furthermore, using our complementation system, this necessitates the catalytic activity of Dbp7 (Figure 5D). Collectively, our data supports a remodeling function of Dbp7 to facilitate both the release and recruitment of early AF as well as the stable incorporation of Rlp3 necessary for maturation of very early pre-60S, and this requires the enzymatic function of Dbp7. 


\section{DISCUSSION}

Our understanding of the very early stages of large subunit assembly remains to be challenged by its anticipated highly dynamic nature coupled with the poorly understood roles of the associated assembly factors such as RNA helicases. In yeast, the RNA helicases Dbp3, Dbp6, Dbp7, Dbp9, Has1, Mak5, Prp43 and Drs1 are implicated in the very early assembly stages $^{48,50,51}$. Many of these RNA helicases are not present in the recent available cryo-EM structures of nucleolar pre-60S particles ${ }^{15-17}$ and their molecular functions remain largely unexplored. In this work, we employ a combination of biochemical and molecular methods to further characterize the function of the non-essential DEAD box RNA helicase Dbp7 after its initial reported role in large sub-unit biogenesis ${ }^{30}$. This led us to uncover the role of Dbp7 in the very early stages of large sub-unit biogenesis and gain insights into some key events during this maturation stage. Taking all our data together, we propose a model where the remodeling function of Dbp7 at the domain VI triggers the release of snR190 concomitant with the exit of Npa1 (complex) from early pre-60S particle, and this in turn facilitate the stable incorporation of ribosomal protein uL3 to bridge domains I and VI and drive the compaction of the nascent pre-60S particle (see further discussion below).

The detection of Dbp7 crosslinking sites in H98-99 of domain VI is indicative of an early particle as these helices are already folded in the earliest so-called state A nucleolar particle ${ }^{15}$. This identified crosslinking site is also consistent with the reported gradual, co-transcriptional association of AFs into the pre-rRNA with Dbp7 being recruited only after complete transcription of the $35 \mathrm{~S}$ pre-rRNA ${ }^{52}$. Furthermore, Dbp7 associates mainly with 27SA pre-rRNA indicating an intermediate particle closely downstream of the earliest pre-60S. Consistently, snoRNP components such as Cbf5, Nop1, Nop58 and Nop56 are present in Dbp7-contaning particle. This multiple line of evidences strongly indicates the very early recruitment and function of Dbp7 in large subunit biogenesis.

The identification of AFs in Dbp7-containing pre-60S particle highly identical to factors associated with Npa1-containing particle ${ }^{18}$ suggests a function of Dbp7 in related events of early biogenesis. The Npa1 complex consisting of Npa1, Npa2, Nop8, and Rsa3, in cooperation with the DEAD-Box RNA helicase Dbp6, has been shown to have key structural function to mediate very early inter-domain interactions leading to the compaction of the nascent particle ${ }^{8}$. Similar to Dbp7, all members of this complex are associated with 27SA prerRNA, and the stable association of Npa1 and Npa2 also requires the full transcription of 25S rRNA $^{52}$. Moreover, synthetic lethality between certain mutant alleles demonstrates a genetic interaction between Dbp6 and Dbp $7^{30}$. This line of evidences suggest that Dbp7 may have a distinct but tied function with the Npa1 complex.

In contrast to the Npa1-containing particle, the absence of factors binding to $90 \mathrm{~S}$ particle such as Enp2, Kri1, Utp9 and Nsr1, and the evident association of AFs such as Ssf1, Rlp24, Nog1, 
Mrt4, which are associated with both early and late pre-60S complexes ${ }^{15,53-55}$, imply that Dbp7 associates to the early pre-60S particle albeit later than Npa1. This provides the basis for the chorological order of events proposed in our model (further expound below). Moreover, Dbp7 crosslinking site in H98-99 is identical to one of the identified binding sites of Npa1 ${ }^{8}$. Hence, it makes a considerable sense that Npa1 is first expelled from H98-99, most likely with the aid of Dbp6 enzymatic function, and this region is then taken over by Dbp7. This also opens the possibility that Dbp7 is necessary for the subsequent exit of Npa1 complex from the pre-60S particle. These speculations are strongly supported by the observed accumulation of Npa1 and its associated proteins in Nop2-contaning particle when Dbp7 is lacking.

Aside from the Npa1 complex present in Dbp7-containing particle, the identification of Rrp5, Mak21, Noc2, and Nop4, all of which have multiple RNA-binding motifs predicted to form rigid scaffolds ${ }^{20,56,57}$, suggests the involvement of Dbp7 in the initial folding of the pre-rRNA for subsequent compaction of the nascent $60 \mathrm{~S}$. This is further supported by the evident association in Dbp7-containing particle of the ribosomal $\mathrm{uL3}$, which is critical for stabilization of early large subunit intermediates ${ }^{12,58}$. In the mature yeast large subunit, uL3 binds in both $5^{\text {' }}$ and $3^{\prime}$ termini ${ }^{11}$. This involves the short helices corresponding to the root helices of the structural domains I and VI of 25S rRNA. In the final architecture of the 60 S subunit, these short helices, together with those of other domains, are all brought together. There is an increasing number of evidence form cryo-EM structures and high-throughput structure probing experiments indicating that the initial compaction of the earliest pre-60S involves the proper folding and subsequent clustering of domains I, II, and $\mathrm{VI}^{15-17,59}$. In the so-called state $\mathrm{B}$ nucleolar particle, where compaction and clustering of domains I, II and VI are evident, UL3 can already be resolved hence, supporting its role in the initial compaction and stabilization of early 605 intermediates. In a succeeding nucleolar particle state C, uL3 interacts with root helix of domain $\mathrm{VI}$ and is in close proximity to the binding site of Dbp7. This observation, together with the notable enrichment of UL3 in Dbp7-containing particle and the marked decrease of uL3 in Nop2-containing particle when Dbp7 is lacking, strongly suggests a functional link between the Dbp7 and uL3. We propose in our model that subsequent binding of Dbp7 in H9899 of domain VI after Npa1 facilitates the initial docking of uL3. This proposed preliminary interaction of uL3 is consistent with the gradual secondary and tertiary structure formation in the domain VI including uL3 binding region as nucleolar pre-60S particles mature, and likewise, the absence of internal segment of uL3 in earlier state B and C nucleolar particles ${ }^{15}$. Although genetic interactions were demonstrated between members of Npa1 complex and $\mathrm{LL}^{31}$, a recent work failed to show the role of Npa1 complex in the recruitment or stable integration of $\mathrm{uL}^{8}$. Our findings fill in this gap and are consistent to the hypothesis that Npa1 may be maintaining the clustering of the root helices prior to uL3. Additionally, our data strongly argue that the stable incorporation of uL3 necessitates the catalytic activity of Dbp7 (see further 
discussion below). However, we cannot exclude the possible contributions of Dbp6 and Dbp9, which also genetically and physically interacts with the Npa1 complex ${ }^{18,31,32}$.

Interestingly, a crosslinking site in $\mathrm{H} 89$ of domain $\mathrm{V}$ was also identified by our CRAC experiment. When mapped to state $C$ particle, this crosslinking site cluster together with the other two identified crosslinking sites in domain VI strongly indicating a binding platform instead of two separate binding events. This characteristic three-dimensional clustering of remote crosslinking sites has also been observed for RNA helicase Has $1^{27}$. The H89 is part of the peptidyl transferase center (PTC), which harbors a number of modifications. Accordingly, our CRAC data and Dbp7-TAP pulldown revealed relative enrichment of reads for snoRNAs that guide modifications around this area. However, CLASH (crosslinking, ligation and analysis of sequence hybrids) analysis did not yield considerable snoRNA-rRNA hybrids to support direct interaction (data not shown). Hence, this rather suggests a steric interaction of Dbp7 relevant for targeting its cognate RNA substrate in H98-99. Among the snoRNAs enriched, snR190 showed the strongest association with Dbp7-containing particle. This snoRNA has predicted 2'-O-methylation site at G2395 residue of the 25S rRNA but current high-throughput methods have never detected this modification ${ }^{34,60,61}$. Recently, a complementary sequence targeting a region of domain I has been identified ${ }^{8}$ suggesting that it has instead a folding-chaperon function to bring domains I and V together. Such snoRNA-mediated RNA folding in ribosome biogenesis has also been exemplified by the U3 snoRNP ${ }^{62-65}$. The observed accumulation of snR190 in Nop2-containing particle and 60s pre-ribosomal fractions when Dbp7 is lacking suggest the involvement of Dbp7 in the release of snR190. We further propose in our model that the remodeling activity of Dbp7 inflicts an indirect, nearby effect on the PTC region. This is supported by the similar observed accumulation of other snoRNAs modifying this region including snR34, which has a modification sites directly within the identified Dbp7 crosslinking site in domain $\mathrm{V}(\mathrm{H} 89)$. Moreover, this remodeling activity may be coupled with ATP hydrolysis and subsequent release of Dbp7 to trigger a localized, concomitant release of proximal snoRNPs. Taking into consideration that snR190 is bound by Npa1 particularly within the region that interacts with domain $V$ of the pre-rRNA ${ }^{8}$, it is very likely that this energy-driven rearrangement also triggers the release of Npa1 from the pre-60S particle. The release of snR190 and Npa1 may then facilitate the stable incorporation of uL3 through its subsequent interaction with domain I following domain VI. This makes considerable sense as the binding sites of snR190 and Npa1 in domain I are in very close proximity with the binding site of uL3 in mature $60 \mathrm{~S}^{8,11}$. Furthermore, this corroborates well with the observed requirement of Dbp7's catalytic activity for UL3 recruitment. This proposed series of events is strongly supported by the available cryo-EM structures of early nucleolar intermediates of the large subunit ${ }^{15,16}$. For example, in state $\mathrm{B}^{15}$ when we anticipate initial binding of $\mathrm{UL} 3$ occurs, domain $\mathrm{V}$ remains flexible and unfolded, and thus highly likely allows snoRNPs to associate and install 
modifications. However, domain $\mathrm{V}$ may still be tethered provided by the interaction with snR190 and Npa1. The energy-driven remodeling of domains $\mathrm{VI}$ and $\mathrm{V}$ by Dbp7 then results to the release of Dbp7, Npa1, snoRN190, and snoRNPs. This in turn triggers the folding of domain $\mathrm{V}$ (helices $\mathrm{H} 89-\mathrm{H} 91$ ) as the particle progresses to state $\mathrm{C}^{15}$ and presumably subsequent inter-domain binding of uL3 to mediate further clustering of domains and hence, compaction of the early pre-60S particle.

Consistent with an earlier study ${ }^{30}$, the absence of Dbp7 affects the processing and/or stability of 27SA. Protein composition of Dbp7-TAP revealed the association of AF and ribosomal proteins that upon their depletion also elicit the same pre-rRNA processing defect. Furthermore, compositional analysis of pre-60S in cells lacking Dbp7 revealed a substantial decrease of "A3" factors, which substantiate the observed pre-rRNA processing defect. This decrease, particularly for Cic1 and Nop15, may be linked to the retention of Npa1 which also binds to ITS1 close to the binding site of these two assembly factors ${ }^{8}$. Decrease in "A3" factors have also been observed upon depletion of ribosomal protein uL30 and $\mathrm{eL} 8^{66}$, which bind to domains I and II, respectively, and facilitate the folding of these regions. In the case of domain VI, architectural support on this region is likely governed by uL3. Cryo-EM structures of early nucleolar pre-60S particles reveals compaction of domains I, II, and VI chaperoned by the binding of $\mathrm{A} 3$ factors and ribosomal proteins around these rRNA domains ${ }^{15,16}$. This highlights a structural requirement and not only complete transcription of 25S rRNA for ITS1 processing to occur, consistent with $\mathrm{A} 3$ factors lacking enzymatic function. Furthermore, this demonstrates that proper folding of domains I, II and VI promotes stable incorporation of A3 factors and vice versa, which in turn may serve as final structural checkpoint before irreversible pre-rRNA processing occurs. This exemplifies how r-proteins assembly events, transient association of AFs and pre-rRNA maturation are functionally linked in the course of biogenesis.

Lastly, the identification of other early-binding RNA helicases including Dbp3, Dbp9, Dbp6, Mak5, and Has1 underlines an intricate conformational rearrangement of the pre-RNA and associated proteins necessary to achieve proper pre-rRNA folding and initial compaction. Hence, this support the current view that the earliest stages might involve many concurrent transitions to reduce multivalent interactions and conformational freedom of the pre-rRNA ${ }^{13}$. One can imagine this as an internal RNA-protein phase separation in the already phase separated nucleolus, a process that is likely to be highly dynamic and heterogeneous. Molecular snapshots of this complex maturation pathway of the earliest pre-60S particle might be provided shortly by recent advances in in situ cryo-EM tomography. Nevertheless, our findings and proposed model align with our limited, existing knowledge of the very early stages of large subunit biogenesis. 


\section{METHODS}

\section{Molecular cloning}

The DBP7 coding sequence (CDS) was amplified with a primer set listed in Supplementary Table S4 and cloned into a PQE80-derivative vector for recombinant expression of Dbp7 with $\mathrm{N}$-terminal His10-ZZ tag in Escherichia coli (E. coli). For yeast complementation system, the ORF including 500 nucleotides upstream the start codon and downstream the stop codon was amplified (Suppl. Table S4) and cloned into pRS415 vector to maintain endogenous expression level. To generate the Dbp7DQGD mutant, each generated wild-type plasmid was subjected to site-directed mutagenesis using primers to introduce a point mutation $(Q \rightarrow G)$ in glutamate E309 within the DEAD conserved sequence motif. All plasmid constructs used and generated in this study are listed in Supplementary Table S5.

\section{Yeast strains and growth analysis}

All yeast strains generated in this study are based on Saccharomyces cerevisiae BY4171 and are listed in Supplementary Table S6. The $\Delta d b p 7$ yeast strain was obtained from the $S$. cerevisiae deletion collection (Euroscarf). Yeast strains harboring proteins with $\mathrm{C}$-terminal His6-TEV protease cleavage site-Protein A (HTP) tag, Calmodulin-binding peptide-TEV protease cleavage site-Protein A (TAP) tag, yeast-enhanced GFP Tag, 3xHA-tag, or under pGals promoter including an N-terminal HA-tag, in their genome were generated according to standard protocols using primers listed in Supplementary Table S4. Yeast complementation system were generated by transforming deltaDBP7 or pGalsDBP7 yeast strain with pRS145derived plasmids (Supplementary Table S5) using a standard transformation protocol. For fluorescence microscopy, strains harboring genomically encoded Dbp7 with c-terminal GFP tag were transformed with plasmid encoding Nop1-RFP. All yeast cultures were grown in Yeast Complete medium ( $1 \%$ yeast extract, $2 \%$ peptone/tryptone, $2 \%$ glucose or galactose) or synthetic medium lacking one of the amino acid $(1.9 \mathrm{~g} / \mathrm{L}$ yeast nitrogen base without amino acid (Formedium), $5 \mathrm{~g} / \mathrm{L}$ ammonium suphate, 2\% dextrose, and corresponding amount of complete supplemented dropout of -HIS/-LEU/-URA (Formedium)). For all experiments, cultures were propagated for $>12$ hours in exponential phase before harvesting. Cell growth was monitored every 90 or $120 \mathrm{~min}$ in liquid culture or through spotting of ten-fold serial dilutions of cultures in agar medium followed by examination after 2 or 3 days. For transient depletion of Dbp7, yeast strain with DBP7 under a pGals promoter growing exponentially in medium containing $2 \%$ galactose were subsequently grown under $2 \%$ glucose for 12 hours before harvesting. 


\section{RNA isolation and northern blotting}

RNA was extracted from yeast cells, sucrose density gradient fractions and pre-60S particles immobilized on IgG sepharose as previously described previously described ${ }^{26,27,67}$ with few modifications. For analysis of pre-rRNA processing, $6 \mu \mathrm{g}$ of extracted RNAs were denatured in glyoxal dye at $55^{\circ} \mathrm{C}$, separated on a $1.2 \%$ ultrapure agarose in 1 X BPTE (10 mM PIPES, $20 \mathrm{mM}$ Bis-Tris, $10 \mathrm{mM}$ EDTA) at 60V for $16 \mathrm{hr}$, and then transferred through vacuum blotting onto a nylon membrane. For analysis of pre-RNAs and snoRNAs present in Dbp7- or Nop2associated pre-60S particle, RNAs were extracted directly from the IgG beads and were separated in $1.2 \%$ agarose gel for pre-rRNAs detection or in $8 \%$ denaturing PAGE (7M Urea) for snoRNA analysis. For extraction of RNA from sucrose density centrifugation fractions to check for snoRNA distribution, each fraction was added with $265 \mu \mathrm{L}$ of GTC mix (6 M guanidinium thiocyanate, $75 \mathrm{mM}$ Tris/ $\mathrm{HCl} \mathrm{pH} \mathrm{8,} 0.2 \mathrm{M} \beta$-mercaptoethanol, $3 \%$ sarkosyl) and $530 \mu \mathrm{L}$ of $\mathrm{PCl}$ (25:24:1), vortexed, and centrifuge at max speed for $15 \mathrm{~min}$. Four hundred microliters of the resulting supernatant were then added with $40 \mu \mathrm{L}$ of $3 \mathrm{M} \mathrm{NaAc}, 2 \mu \mathrm{L}$ of GlycogenBlue (Invitrogen), $1.2 \mathrm{~mL}$ of $100 \%$ ethanol, and precipitated overnight at $-20{ }^{\circ} \mathrm{C}$. Extracted RNAs per fraction were separated on 8\% denaturing PAGE (7M Urea) in 1X TBE at 30 watts and blotted onto a nylon membrane at $60 \mathrm{~V}$ for $2 \mathrm{hrs}$ in $0.5 \mathrm{X}$ TBE. RNA targets were detected using corresponding 5'-[32P]-labelled DNA oligonucleotides listed in Supplementary Table S4. Membranes were exposed to phosphoimager screens and radioactive signals were detected using Typhoon FLA 9500 (GE Healthcare Limited) phospoimager. Images were quantified using ImageStudio ${ }^{\mathrm{TM}}$ Lite (LI-COR Biosciences) or ImageQuant (GE Healthcare Limited) software. Membranes for re-probing were stripped using boiled Stripping Buffer $(0.1 \mathrm{X}$ SSC, $0.1 \%$ SDS) at $70{ }^{\circ} \mathrm{C}$ for $1 \mathrm{~h}$.

\section{Isolation of pre-ribosomal particles}

Complexes associated with TAP-tagged proteins were isolated as previously described ${ }^{27}$ with minor modifications. Cells were lysed by grinding in liquid nitrogen in a buffer containing $50 \mathrm{mM}$ Tris- $\mathrm{HCl} \mathrm{pH} \mathrm{7.8,} 150 \mathrm{mM} \mathrm{NaCl}, 1.5 \mathrm{mM} \mathrm{MgCl}_{2}, 0.05 \% \mathrm{NP}-40,2 \mathrm{mM}$ DTT and protease inhibitors. Clarified lysate were incubated with IgG sepharose beads for $2 \mathrm{hr}$ to capture proteinA-tagged proteins. After subsequent washing, bound complexes were eluted with TEV protease overnight at $4{ }^{\circ} \mathrm{C}$. Proteins in the eluate were then precipitated using $25 \%$ TCA and analysed through western blotting using the antibodies listed on Supplementary Table 7 or by mass spectrometry. For analysis of co-purified RNAs by northern blotting, RNA were extracted and detected as described above. 


\section{Sucrose density gradient centrifugation}

Exponentially growing cells were lysed as described above for isolation of pre-ribosomal particles. $350 \mu \mathrm{L}$ of cleared lysate were separated on $10-45 \%$ sucrose density gradient in an SW-40Ti rotor for $16 \mathrm{~h}$ at 23,500 rpm as previously described ${ }^{26} .530 \mathrm{uL}$ was collected for each fraction and the absorbance at $260 \mathrm{~nm}$ was measured using NanoDrop ${ }^{\mathrm{TM}}$ (Thermo Scientific). $265 \mu \mathrm{L}$ of each fraction were precipitated with $20 \%$ TCA and the extracted proteins were analyzed by western blotting. RNAs present in the remaining half of each fraction were extracted and subsequently analyzed by northern blotting.

\section{Crosslinking and analysis of cDNA (CRAC)}

UV crosslinking and analysis of cDNA (CRAC) experiments were done as previously described ${ }^{37-39}$ with few adaptations. Wild-type cells and yeast strains expressing genomically encoded, HTP-tagged Dbp7 were first grown exponentially in low uracil medium $(10 \mathrm{mg} / \mathrm{L}$ uracil) supplemented with $100 \mu \mathrm{M}$ 4-thiouracil until OD600 of 0.5. After which, they were grown in the presence of $1 \mathrm{mM} 4$-thiouracil for additional $4 \mathrm{~h}$ prior to harvesting and crosslinking at $365 \mathrm{~nm}$ using 2 rounds of $600 \mathrm{~mJ} / \mathrm{cm}^{2}$ in a Stratalinker (Agilent Technologies). RNA-protein complexes were subsequently isolated, and cDNA library for Illumina deep sequencing were prepared as previously described. Sequencing reads were subjected to processing and quality control, and only those containing a T-C mutation were mapped to the S. cerevisiae genome (S228C) using Bowtie 2 (ver 2.2.4). The distribution of reads across different RNA species was determined using pyCRAC read counting ${ }^{68}$. An in-house python script was then used to map the reads onto available secondary structures of rRNA ${ }^{41}$ and tertiary structure of the so-called State $C$ early pre-60S particle ${ }^{15}$ using a color scale ${ }^{69}$.

\section{Proteomics analysis and data processing}

Isolated pre-ribosomal proteins were separated by SDS-PAGE, and each lane was cut into 21 slices. All gel slices were reduced, alkylated, and then digested with modified trypsin. The resulting peptides were extracted from the gel and vacuum-dried.

Dried peptides were dissolved in $2 \%$ acetonitrile (ACN) containing $0.1 \%$ formic acid (FA) and analyzed using a $Q$ Exactive HF mass spectrometer (ThermoFisher Scientific) coupled with an Ultimate 3000 RSLC system (Dionex). Peptides were loaded on a reverse-phase C18 precolumn (Dionex, $5 \mathrm{~mm}$ long, $0.3 \mathrm{~mm}$ inner diameter), and desalted for 3 minutes using buffer A $(0.1 \% \mathrm{FA})$. After 3 minutes, the pre-column was switched online with a self-made analytical column (30 cm long, $75 \mu \mathrm{m}$ inner diameter, packed with $1.9 \mu \mathrm{m}$ ReproSil-Pur C18-AQ beads (Dr. Maisch $\mathrm{GmbH})$ ). Trapped peptides were separated with a linear gradient of $5-45 \%$ buffer $\mathrm{B}(80 \% \mathrm{FA}$ and $0.1 \% \mathrm{FA})$ at a flow rate of $300 \mathrm{nl} / \mathrm{min}$. The total run time was $58 \mathrm{~min}$. Both the pre-column and the column were maintained at $50^{\circ} \mathrm{C}$. The $\mathrm{Q}$ Exactive $\mathrm{HF}$ was operated in a 
data-dependent acquisition manner where one full MS scan across the $350-1600 \mathrm{~m} / \mathrm{z}$ range was acquired at a resolution setting of 60,000 FWHM (full width, half maximum) to select up to 30 most abundant peptide precursors. Precursors were fragmented by Higher Collision Energy Dissociation (HCD) with nitrogen at a normalized collision energy setting of $28 \%$, and their product ion spectra were recorded at resolution of 15,000 FWHM with the maximum ion injection time of $60 \mathrm{~ms}$.

The MS raw files were processed by MaxQuant ${ }^{70}$ (version 1.6.5.0) and MS/MS spectra were searched against UniProt S. Cerevisiae database (downloaded on Feb 2019 with 9731 entries) using default settings. Trypsin was used for protein digestion with up to two mis-cleavages. Methionine oxidation and cysteine carbamidomethylation were defined as variable and fixed modifications, respectively. The false discovery rate (FDR) was set to $1 \%$ for both peptide and protein identifications. Subsequent data analysis was conducted with Perseus ${ }^{71}$ (version 1.6.2.3). After removing all decoy hits and potential contaminant entries, proteins identified only with modified peptides were filtered out. MaxLFQ intensities ${ }^{72}$ were used for label-free protein quantification. Missing values in replicates were imputed using default settings. A simple T-test was performed, and $p$-values were corrected using the Benjamini-Hochberg method (FDR <0.05).

\section{Purification of recombinant proteins and in vitro ATPase assays}

His-ZZ-tagged recombinant proteins were purified as previously described ${ }^{73}$ with few changes. Following induction of expression in E. coli (BL21 Codon Plus) with $1 \mathrm{mM}$ overnight at $18{ }^{\circ} \mathrm{C}$, cells were pelleted, resuspended in lysis buffer $(50 \mathrm{mM}$ Tris- $\mathrm{HCl} \mathrm{pH} \mathrm{7,500} \mathrm{mM} \mathrm{NaCl,} 1 \mathrm{mM}$ $\mathrm{MgCl}_{2} 10 \mathrm{mM}$ imidazole, $1 \mathrm{mM}$ PMSF, $10 \%$ glycerol), and lysed by sonication. Cleared cell lysate was added with polyethyleneimine to a final concentration of $0.05 \%$ to remove proteinassociated nucleic acids. The resulting soluble fraction was then incubated with cOmplete HisTag purification resin (Roche). Following thorough washing first with low and then high salt buffer $(50 \mathrm{mM}$ Tris-HCl pH 7.0, 500/1000 mM NaCl, 1 mM MgCl $2,30 \mathrm{mM}$ imidazole, $10 \%$ glycerol), bound proteins were eluted with a buffer containing $50 \mathrm{mM}$ Tris- $\mathrm{HCl} \mathrm{pH} \mathrm{7,500} \mathrm{mM}$ $\mathrm{NaCl}, 1 \mathrm{mM} \mathrm{MgCl}$, $300 \mathrm{mM}$ imidazole and 10\% glycerol. Fractions containing proteins were pooled and dialysed against a buffer containing $50 \mathrm{mM}$ Tris- $\mathrm{HCl} \mathrm{pH} \mathrm{7,120} \mathrm{mM} \mathrm{NaCl,} 2 \mathrm{mM}$ $\mathrm{MgCl}_{2}, 20 \%$ glycerol. The quantity of the purified protein was measured using a Bradford assay and the quality is assessed by SDS-PAGE followed by Coomassie staining.

NADH-coupled ATPase assays were essentially performed as previously ${ }^{27,74,75}$. Reactions were carried out in solution containing $50 \mathrm{mM}$ Tris- $\mathrm{HCl} \mathrm{pH}$ 7.4, $25 \mathrm{mM} \mathrm{NaCl}, 2 \mathrm{mM} \mathrm{MgCl}$, 4 mM ATP, 1.5 mM PEP (phosphoenolpyruvate), $450 \mu \mathrm{M} N A D H, 1.5 \mu \mathrm{M}$ recombinantly expressed and purified RNA helicase, and $1.5 \mu \mathrm{M}$ RNA ((32 nt; 5'GUAAUGAAAGUCCAUGUAAAACAAAACAAAAC-3'). Absorbance was measure every 50 
seconds for 30 min at $37{ }^{\circ} \mathrm{C}$ using a Gen5 Microplate Reader (Biotek) and the rate of ATP hydrolysis was calculated using the following equation:

$$
\text { nM ATP hydolysis } x \mathrm{sec}^{-1}=\frac{d A_{340}}{d t} \times K_{\text {path }}^{-1} \times 10^{6}
$$




\section{DATA AVAILABILITY}

The CRAC datasets and their analyses for Dbp7-HTP and the wild-type yeast control are deposited in Gene Expression Omnibus (GEO) database [http://www.ncbi.nlm.nih.gov/geo/] under the accession code GSE160734. The RMS dataset present here are also deposited in the GEO data base under the accession code GSE161347. Proteomic data are deposited in the PRIDE database [https://www.ebi.ac.uk/pride/] with the accession code PXD022625. Other data supporting the findings of this study are available from the corresponding authors upon reasonable request 


\section{REFERENCES}

1. Grandi, P. et al. 90 S pre-ribosomes include the 35 S pre-rRNA, the U3 snoRNP, and $40 \mathrm{~S}$ subunit processing factors but predominantly lack $60 \mathrm{~S}$ synthesis factors. Mol. Cell 10, 105-115 (2002).

2. Henras, A. K., Plisson-Chastang, C., O'Donohue, M.-F., Chakraborty, A. \& Gleizes, P.-E. An overview of pre-ribosomal RNA processing in eukaryotes. Wiley Interdiscip. Rev. RNA 6, 225-242 (2015).

3. Gerhardy, S., Menet, A. M., Peña, C., Petkowski, J. J. \& Panse, V. G. Assembly and nuclear export of pre-ribosomal particles in budding yeast. Chromosoma 123, 327344 (2014).

4. Sloan, K. E. et al. Tuning the ribosome: The influence of rRNA modification on eukaryotic ribosome biogenesis and function. RNA Biol. 1-16 (2016) doi:10.1080/15476286.2016.1259781.

5. Watkins, N. J. \& Bohnsack, M. T. The box C/D and H/ACA snoRNPs: key players in the modification, processing and the dynamic folding of ribosomal RNA. Wiley Interdiscip. Rev. RNA 3, 397-414 (2012).

6. Bohnsack, K. E. \& Bohnsack, M. T. RNA-Binding Proteins Chaperone Ribonucleoprotein Complex Assembly to Solve the RNA-Folding Problem. Cell 179, 1248-1250 (2019).

7. Duss, O., Stepanyuk, G. A., Puglisi, J. D. \& Williamson, J. R. Transient Protein-RNA Interactions Guide Nascent Ribosomal RNA Folding. Cell 179, 1357-1369.e16 (2019).

8. Joret, C. et al. The Npa1p complex chaperones the assembly of the earliest eukaryotic large ribosomal subunit precursor. PLOS Genet. 14, e1007597 (2018).

9. Ojha, S., Malla, S. \& Lyons, S. M. snoRNPs: Functions in Ribosome Biogenesis. Biomolecules 10, 783 (2020).

10. Rodgers, M. L. \& Woodson, S. A. Transcription Increases the Cooperativity of Ribonucleoprotein Assembly. Cell 179, 1370-1381.e12 (2019).

11. Ben-Shem, A. et al. The Structure of the Eukaryotic Ribosome at $3.0 \AA$ Resolution. Science 334, 1524-1529 (2011).

12. Gamalinda, M. et al. A hierarchical model for assembly of eukaryotic 605 ribosomal subunit domains. Genes Dev. 28, 198-210 (2014).

13. Klinge, S. \& Woolford, J. L. Ribosome assembly coming into focus. Nat. Rev. Mol. Cell Biol. 20, 116-131 (2019).

14. Konikkat, S. \& Woolford, J. L. Principles of 60 S ribosomal subunit assembly emerging from recent studies in yeast. Biochem. J. 474, 195-214 (2017). 
15. Kater, L. et al. Visualizing the Assembly Pathway of Nucleolar Pre-60S Ribosomes. Cell 171, 1599-1610.e14 (2017).

16. Sanghai, Z. A. et al. Modular assembly of the nucleolar pre-60S ribosomal subunit. Nature 556, 126-129 (2018).

17. Zhou, D. et al. Cryo-EM structure of an early precursor of large ribosomal subunit reveals a half-assembled intermediate. Protein Cell 10, 120-130 (2019).

18. Dez, C. et al. Npa1p, a component of very early pre-60S ribosomal particles, associates with a subset of small nucleolar RNPs required for peptidyl transferase center modification. Mol. Cell. Biol. 24, 6324-6337 (2004).

19. Khoshnevis, S. et al. The DEAD-box Protein Rok1 Orchestrates $40 \mathrm{~S}$ and $60 \mathrm{~S}$ Ribosome Assembly by Promoting the Release of Rrp5 from Pre-40S Ribosomes to Allow for 60 S Maturation. PLOS Biol. 14, e1002480 (2016).

20. Lebaron, S. et al. Rrp5 Binding at Multiple Sites Coordinates Pre-rRNA Processing and Assembly. Mol. Cell 52, 707-719 (2013).

21. de la Cruz, J., Karbstein, K. \& Woolford, J. L. Functions of ribosomal proteins in assembly of eukaryotic ribosomes in vivo. Annu. Rev. Biochem. 84, 93-129 (2015).

22. Ohmayer, U. et al. Studies on the Coordination of Ribosomal Protein Assembly Events Involved in Processing and Stabilization of Yeast Early Large Ribosomal Subunit Precursors. PLOS ONE 10, e0143768 (2015).

23. Pöll, G. et al. rRNA Maturation in Yeast Cells Depleted of Large Ribosomal Subunit Proteins. PLOS ONE 4, (2009).

24. Rosado, I. V. \& Cruz, J. D. L. Npa1p is an essential trans-acting factor required for an early step in the assembly of 605 ribosomal subunits in Saccharomyces cerevisiae. RNA 10, 1073-1083 (2004).

25. Martin, R., Straub, A. U., Doebele, C. \& Bohnsack, M. T. DExD/H-box RNA helicases in ribosome biogenesis. RNA Biol. 10, 4-18 (2013).

26. Bohnsack, M. T. et al. Prp43 Bound at Different Sites on the Pre-rRNA Performs Distinct Functions in Ribosome Synthesis. Mol. Cell 36, 583-592 (2009).

27. Brüning, L. et al. RNA helicases mediate structural transitions and compositional changes in pre-ribosomal complexes. Nat. Commun. 9, 5383 (2018).

28. Dembowski, J. A., Ramesh, M., McManus, C. J. \& Woolford, J. L. Identification of the binding site of Rlp7 on assembling 60S ribosomal subunits in Saccharomyces cerevisiae. RNA 19, 1639-1647 (2013).

29. Daugeron, M. C., Kressler, D. \& Linder, P. Dbp9p, a putative ATP-dependent RNA helicase involved in 60S-ribosomal-subunit biogenesis, functionally interacts with Dbp6p. RNA 7, 1317-1334 (2001). 
30. Daugeron, M. C. \& Linder, P. Dbp7p, a putative ATP-dependent RNA helicase from Saccharomyces cerevisiae, is required for $60 \mathrm{~S}$ ribosomal subunit assembly. RNA N. Y. N 4, 566-581 (1998).

31. Rosado, I. V. et al. Characterization of Saccharomyces cerevisiae Npa2p (Urb2p) Reveals a Low-Molecular-Mass Complex Containing Dbp6p, Npa1p (Urb1p), Nop8p, and Rsa3p Involved in Early Steps of 60S Ribosomal Subunit Biogenesis. Mol. Cell. Biol. 27, 1207-1221 (2007).

32. de la Cruz, J., Lacombe, T., Deloche, O., Linder, P. \& Kressler, D. The putative RNA helicase Dbp6p functionally interacts with Rpl3p, Nop8p and the novel trans-acting Factor Rsa3p during biogenesis of 605 ribosomal subunits in Saccharomyces cerevisiae. Genetics 166, 1687-1699 (2004).

33. Koš, M. \& Tollervey, D. Yeast Pre-rRNA Processing and Modification Occur Cotranscriptionally. Mol. Cell 37, 809-820 (2010).

34. Birkedal, U. et al. Profiling of ribose methylations in RNA by high-throughput sequencing. Angew. Chem. Int. Ed Engl. 54, 451-455 (2015).

35. Krogh, N., Birkedal, U. \& Nielsen, H. RiboMeth-seq: Profiling of 2'-O-Me in RNA. Methods Mol. Biol. Clifton NJ 1562, 189-209 (2017).

36. Wu, S. et al. Diverse roles of assembly factors revealed by structures of late nuclear pre-60S ribosomes. Nature 534, 133-137 (2016).

37. Bohnsack, M. T., Tollervey, D. \& Granneman, S. Identification of RNA helicase target sites by UV cross-linking and analysis of cDNA. Methods Enzymol. 511, 275-288 (2012).

38. Braun, C. M. et al. Pol5 is required for recycling of small subunit biogenesis factors and for formation of the peptide exit tunnel of the large ribosomal subunit. Nucleic Acids Res. 48, 405-420 (2020).

39. Memet, I., Doebele, C., Sloan, K. E. \& Bohnsack, M. T. The G-patch protein NF-KBrepressing factor mediates the recruitment of the exonuclease XRN2 and activation of the RNA helicase DHX15 in human ribosome biogenesis. Nucleic Acids Res. 45, 5359-5374 (2017).

40. Martin, R. et al. A pre-ribosomal RNA interaction network involving snoRNAs and the Rok1 helicase. RNA 20, 1173-1182 (2014).

41. Petrov, A. S. et al. Secondary Structures of rRNAs from All Three Domains of Life. PLOS ONE 9, e88222 (2014).

42. Barrio-Garcia, C. et al. Architecture of the Rix1-Rea1 checkpoint machinery during pre-60S-ribosome remodeling. Nat. Struct. Mol. Biol. 23, 37-44 (2016).

43. Greber, B. J. Mechanistic insight into eukaryotic $60 \mathrm{~S}$ ribosomal subunit biogenesis by cryo-electron microscopy. RNA N. Y. N 22, 1643-1662 (2016). 
44. Ma, C. et al. Structural snapshot of cytoplasmic pre-60S ribosomal particles bound by Nmd3, Lsg1, Tif6 and Reh1. Nat. Struct. Mol. Biol. 24, 214-220 (2017).

45. Malyutin, A. G., Musalgaonkar, S., Patchett, S., Frank, J. \& Johnson, A. W. Nmd3 is a structural mimic of elF5A, and activates the cpGTPase Lsg1 during $60 \mathrm{~S}$ ribosome biogenesis. EMBO J. 36, 854-868 (2017).

46. Zhou, Y., Musalgaonkar, S., Johnson, A. W. \& Taylor, D. W. Tightly-orchestrated rearrangements govern catalytic center assembly of the ribosome. Nat. Commun.10, (2019).

47. Taoka, M. et al. The complete chemical structure of Saccharomyces cerevisiae rRNA: partial pseudouridylation of U2345 in 25S rRNA by snoRNA snR9. Nucleic Acids Res. 44, 8951-8961 (2016).

48. Woolford, J. L. \& Baserga, S. J. Ribosome Biogenesis in the Yeast Saccharomyces cerevisiae. Genetics 195, 643-681 (2013).

49. Babiano, R., Gamalinda, M., Woolford, J. L. \& de la Cruz, J. Saccharomyces cerevisiae Ribosomal Protein L26 Is Not Essential for Ribosome Assembly and Function. Mol. Cell. Biol. 32, 3228-3241 (2012).

50. Dez, C. et al. Npa1p, a Component of Very Early Pre-60S Ribosomal Particles, Associates with a Subset of Small Nucleolar RNPs Required for Peptidyl Transferase Center Modification. Mol. Cell. Biol. 24, 6324-6337 (2004).

51. Rodríguez-Galán, O., García-Gómez, J. J. \& de la Cruz, J. Yeast and human RNA helicases involved in ribosome biogenesis: current status and perspectives. Biochim. Biophys. Acta 1829, 775-790 (2013).

52. Chen, W., Xie, Z., Yang, F. \& Ye, K. Stepwise assembly of the earliest precursors of large ribosomal subunits in yeast. Nucleic Acids Res. 45, 6837-6847 (2017).

53. Fatica, A., Cronshaw, A. D., Dlakić, M. \& Tollervey, D. Ssf1p Prevents Premature Processing of an Early Pre-60S Ribosomal Particle. Mol. Cell 9, 341-351 (2002).

54. Klingauf-Nerurkar, P. et al. The GTPase Nog1 co-ordinates the assembly, maturation and quality control of distant ribosomal functional centers. eLife https://elifesciences.org/articles/52474 (2020) doi:10.7554/eLife.52474.

55. Saveanu, C. et al. Sequential Protein Association with Nascent 60S Ribosomal Particles. Mol. Cell. Biol. 23, 4449-4460 (2003).

56. Granneman, S., Petfalski, E. \& Tollervey, D. A cluster of ribosome synthesis factors regulate pre-rRNA folding and 5.8S rRNA maturation by the Rat1 exonuclease. EMBO J. 30, 4006-4019 (2011).

57. Hierlmeier, T. et al. Rrp5p, Noc1p and Noc2p form a protein module which is part of early large ribosomal subunit precursors in S. cerevisiae. Nucleic Acids Res. 41, 1191-1210 (2013). 
58. de la Cruz, J., Karbstein, K. \& Woolford, J. L. Functions of ribosomal proteins in assembly of eukaryotic ribosomes in vivo. Annu. Rev. Biochem. 84, 93-129 (2015).

59. Burlacu, E. et al. High-throughput RNA structure probing reveals critical folding events during early 60 S ribosome assembly in yeast. Nat. Commun. 8, 714 (2017).

60. Marchand, V., Blanloeil-Oillo, F., Helm, M. \& Motorin, Y. Illumina-based RiboMethSeq approach for mapping of 2'-O-Me residues in RNA. Nucleic Acids Res. 44, e135 (2016).

61. Yang, J. et al. Mapping of Complete Set of Ribose and Base Modifications of Yeast rRNA by RP-HPLC and Mung Bean Nuclease Assay. PLoS ONE 11, (2016).

62. Beltrame, M. \& Tollervey, D. Identification and functional analysis of two U3 binding sites on yeast pre-ribosomal RNA. EMBO J. 11, 1531-1542 (1992).

63. Dutca, L. M., Gallagher, J. E. G. \& Baserga, S. J. The initial U3 snoRNA:pre-rRNA base pairing interaction required for pre-18S rRNA folding revealed by in vivo chemical probing. Nucleic Acids Res. 39, 5164-5180 (2011).

64. Hughes, J. M. Functional base-pairing interaction between highly conserved elements of U3 small nucleolar RNA and the small ribosomal subunit RNA. J. Mol. Biol. 259, 645-654 (1996).

65. Marmier-Gourrier, N., Cléry, A., Schlotter, F., Senty-Ségault, V. \& Branlant, C. A second base pair interaction between U3 small nucleolar RNA and the 5'-ETS region is required for early cleavage of the yeast pre-ribosomal RNA. Nucleic Acids Res. 39, 9731-9745 (2011).

66. Jakovljevic, J. et al. Ribosomal proteins $L 7$ and $L 8$ function in concert with six $A_{3}$ assembly factors to propagate assembly of domains I and II of 25S rRNA in yeast 60S ribosomal subunits. RNA N. Y. N18, 1805-1822 (2012).

67. Gallesio, J. D., Hackert, P., Bohnsack, K. E. \& Bohnsack, M. T. Sgd1 is an MIF4G domain-containing cofactor of the RNA helicase Fal1 and associates with the 5' domain of the 18S rRNA sequence. RNA Biol. 17, 539-553 (2020).

68. Webb, S., Hector, R. D., Kudla, G. \& Granneman, S. PAR-CLIP data indicate that Nrd1-Nab3-dependent transcription termination regulates expression of hundreds of protein coding genes in yeast. Genome Biol. 15, R8 (2014).

69. Sloan, K. E. et al. The association of late-acting snoRNPs with human pre-ribosomal complexes requires the RNA helicase DDX21. Nucleic Acids Res. 43, 553-564 (2015).

70. Cox, J. \& Mann, M. MaxQuant enables high peptide identification rates, individualized p.p.b.-range mass accuracies and proteome-wide protein quantification. Nat.

Biotechnol. 26, 1367-1372 (2008). 
71. Tyanova, S., Temu, T. \& Cox, J. The MaxQuant computational platform for mass spectrometry-based shotgun proteomics. Nat. Protoc. 11, 2301-2319 (2016).

72. Cox, J. et al. Accurate Proteome-wide Label-free Quantification by Delayed Normalization and Maximal Peptide Ratio Extraction, Termed MaxLFQ. Mol. Cell. Proteomics MCP 13, 2513-2526 (2014).

73. Choudhury, P., Hackert, P., Memet, I., Sloan, K. E. \& Bohnsack, M. T. The human RNA helicase DHX37 is required for release of the U3 snoRNP from pre-ribosomal particles. RNA Biol. 16, 54-68 (2019).

74. Heininger, A. U. et al. Protein cofactor competition regulates the action of a multifunctional RNA helicase in different pathways. RNA Biol. 13, 320-330 (2016).

75. Kiianitsa, K., Solinger, J. A. \& Heyer, W.-D. NADH-coupled microplate photometric assay for kinetic studies of ATP-hydrolyzing enzymes with low and high specific activities. Anal. Biochem. 321, 266-271 (2003).

76. Swiatkowska, A. et al. Kinetic analysis of pre-ribosome structure in vivo. RNA 18, 2187-2200 (2012). 


\section{ACKNOWLEDGEMENTS}

This work was supported by the Deutsche Forschungsgemeinschaft (SFB860 to M.T.B. and SFB1190 to K.E.B.) and Göttingen University Medical Department (to M.T.B. and K.E.B.). We thank Lukas Brüning and Jens Kretschmer for help with bioinformatics analysis of PAR-CRAC data.

\section{CONFLICT OF INTEREST}

The authors declare no conflicts of interest.

\section{AUTHOR CONTRIBUTIONS}

G.R.R.A., P.H. and N.K. performed experiments: P.H. performed PAR-CRAC experiments, N.K. performed RMS experiments and rRNA/tRNA expression analyses, and all other experiments were conducted by G.R.R.A. with the support of P.H.. H.U. and K-T.P. contributed to MS analyses, and H.N. to RMS analyses. K.E.B. and M.T.B. conceived and supervised the work. G.R.R.A., K.E.B. and M.T.B. wrote the manuscript. All authors interpreted the data. 


\section{FIGURE LEGENDS}

Figure 1. The catalytic activity of $\mathrm{Dbp} 7$ is required for the conversion of 27SA to 27SB pre-rRNA. (A) Schematic outline of pre-rRNA processing in S. cerevisiae. Mature rRNA sequences are represented as black rectangles, and the internal transcribed spacers (ITS) and external transcribed spacers (ETS) are designated as black lines. Pre-rRNA cleavage sites are indicated by black vertical lines along the 35s pre-rRNA transcript. (B) Northern blot analysis of pre-rRNA processing in WT and $\Delta d b p 7$ strains. Total RNA were extracted and separated in denaturing agarose gel and transferred to membrane. Radio-labelled probes hybridizing in ITS1 and ITS2 were used to detect the indicated pre-rRNA species. Mature 25S and 18S rRNA were visualized by methylene blue (MB) staining. (C) Coomassie-stained SDS gel of recombinant Dbp7 ${ }_{W T}$ and Dbp7DQGD. Equal amounts of recombinantly expressed and purified His-ZZ-tagged proteins were loaded in Nu-PAGE gel followed by coomassie staining. (D) NADH-coupled ATPase assay using recombinant Dbp7 ${ }_{\mathrm{WT}}$ and Dbp7 $7_{\mathrm{DQGD}}$. ATPase activity was monitored using $2.0 \mu \mathrm{M}$ of each purified proteins with or without $1.5 \mu \mathrm{M}$ of RNA. Data is presented as mean \pm standard deviation from three independent measurements. Significance was determined using Student's t-test $\left({ }^{* *}=p<0.01\right.$, n.s. $=$ non-significant). (E) Spot test analysis of growth of $\Delta d b p 7$ complementation system. Equal number of cells from $\Delta d b p 7$ strains complemented with empty pRS415 plasmid (EV) or pRS415-based plasmids for expression of Dbp7 WT $_{\text {T }}$ obp7DQGD were serially diluted (10-folded) and spotted onto a plate containing selective. An equal amount of wild-type strain harbouring an EV was also grown in parallel as a control. Growth was documented after 48 hours of incubation at $30{ }^{\circ} \mathrm{C}$. (F) Pre-rRNA processing in the $d b p 7$ complementation system. The same set of yeast trains from (E) were used for analysis of pre-rRNA processing using the same approach described in (B). The data presented in $B, E$, and $F$ are representative of three independent experiments.

Figure 2. Dbp7 physically associates with early pre-60S complexes. (A) Analysis of Dbp7 association with pre-ribosomal complexes. Whole cell lysate from Dbp7-TAP strains was separated using sucrose density centrifugation. The absorbance at $260 \mathrm{~nm}$ was measured to generate a profile on which the peaks corresponding to (pre)-ribosomal complexes are marked. Total protein was extracted in each fraction and the distribution of Dbp7-TAP, eL15, uL3 were analyzed by western blotting. (B) Analysis of pre-rRNA species associated with Dbp7-TAP particle. Dbp7-containing complexes were pulled down via TAP-tag from wild-type cells or cells expressing Dbp7-TAP and co-purified pre-rRNAs were extracted and visualized by northern blot using [ $\left.{ }^{32} \mathrm{P}\right]$-labelled probes hybridizing in ITS1 and ITS2 region. Input represents $3 \%$ of the lysate used for the pulldown. (C) SDS gel of the proteins present in the eluates of the pulldown similar to (B). Dbp7-containing complexes were purified from yeast total lysate, separated in SDS gel and visualized by coomassie staining. (D) Composition of 
purified Dbp7-TAP particles analyzed by mass spectrometry. Label-Free Quantification (LFQ) intensities were used for analysis in Perseus to identify proteins enriched in Dbp7-containing particles compared to wild-type in the pulldown presented in (C). Shown is a volcano plot (Log p-values vs. Log2 fold difference between the WT control and purified Dbp7-TAP particle) representing one of the two data sets obtained. (E) Western blot analysis confirming the association of uL3. Pulldown was performed as in (C) and the Dbp7-TAP bait as well as uL3, eL15 and uS11 were detected in input and eluate samples by western blotting using an antiPAP antibody and antibodies against the endogenous r-proteins. Input represents $2.5 \%$. All experiments presented in $A, B$ and $E$ were done in triplicate and a representative data were shown.

Figure 3. Dbp7 crosslinks to domain V and VI of the 25S rRNA sequence. (A) Overview of sequencing reads mapping to different RNA species. The relative distribution of reads among the different RNA species after mapping to yeast genome in wild-type (WT) and Dbp7HTP PAR-CRAC samples are presented as pie charts. Highlighted in red and blue are the proportion of reads mapping to RDNA locus and snoRNAs genes respectively. Shown here are two independent PAR-CRAC data sets. (B) Profile of Dbp7 PAR-CRAC data on RDNA locus. The normalized number of reads mapping to each nucleotide of RDN37, which encodes for the 35S pre-rRNA transcript, in Dbp7-HTP and wild-type (WT) sample is presented in the upper panel. The normalized number of the T-to-C mutations mapping to each nucleotide of $R D N 37$ is shown in the lower panel. A schematic representation of $35 \mathrm{~s}$ rRNA is shown at the bottom with black rectangles corresponding to the mature rRNAs while black lines representing the transcribed spacers. (C) A magnified view of the doimain $V$ and VI region of the 25S rRNA secondary structure containing the three major Dbp7-HTP cross-linking sites is shown. The number of sequencing reads mapping to each nucleotide of 25s rRNA is represented by a colour code where the maximum number of reads (100\%) is shown in red and lower numbers of reads $(20 \%)$ are shown in orange-yellow. The predicted basepairing site of snoRNA190 is indicated by purple line, and the domain $\mathrm{V}$ and $\mathrm{VI}$ root helices are indicated in blue and cyan respectively. (D) Modelling of the identified crosslinking sites onto a tertiary structure of state C early pre-60S particle purified via TAP-tagged Nsa1 followed by Flag-tagged Ytm1 (PDB: 6EM1) using a color scale as in (C). The density corresponding to uL3 is colored in green.

Figure 4. A subset of snoRNAs accumulates on pre-60S particles in the absence of Dbp7. (A) Graphical representation of enriched snoRNAs in Dbp7HTP1 data set. The fold enrichments of snoRNAs showing more than two-fold enrichment in Dbp7-HTP_1 PAR-CRAC data set compared to WT_1. (B) SnoRNAs present on Dbp7-TAP particle. Dbp7-TAP particle were captured from cell lysate through IgG beads and co-purified RNAs were extracted. After 
separation by denaturing PAGE, the indicated snoRNAs were detected by northern blot analysis using [32P]-labelled probes hybridizing to each snoRNA. (C) Distribution of snoRNAs on pre-ribosomal complexes of WT and $\Delta d b p 7$ strains. Whole cell lysates were separated by sucrose density gradient centrifugation. The absorbance at $260 \mathrm{~nm}$ was measured to generate profiles on which the peaks corresponding to (pre-)ribosomal complexes are marked. RNAs and proteins were extracted from each sucrose gradient fraction. The distribution of the indicated snoRNAs was analyzed as in (B). Protein samples were separate by denaturing PAGE and western blot analysis using an antibody against endogenous eL15 was performed. (D) Enrichment of snoRNAs in Nop2-TAP particle of WT and $\Delta d b p 7$ strains. Extracts from yeast strains expressing Nop2-TAP in a wild-type (WT) or $\Delta d b p 7$ background, or wild-type yeast not expressing a TAP-tagged protein were used for pulldowns on IgG sepharose. RNAs present in input (3\%) and eluates were extracted and analyzed as in (B). (E) The number of sequencing reads mapping to each nucleotide of snR190 gene in the Dbp7-HTP_2 and wildtype (WT_2) PAR-CRAC data set are shown as indicated. The normalized number of T-C misincorporations corresponding to each nucleotide are presented in the lower graph. A schematic representation of snR190 is shown below with the positions of evolutionarily conserved sequences (boxes) highlighted with black rectangles. All experiments described were done in triplicate and only a representative data is shown.

Figure 5. Dbp7 is required for the recruitment of uL3 to pre-ribosomes. (A-B) Compositional changes in Nop2-TAP particle when Dbp7 is lacking. Nop2-TAP particles were isolated from whole cell lysate of wild-type (WT) and $\Delta d b p 7$ cells through IgG beads and eluted by overnight TEV cleavage. Associated proteins were separated in PAGE gels and subjected to MS. A heat map was generated from two independent biological replicates and shows the relative enrichment (log2 fold-change $\Delta d b p 7$ vs. WT) of assembly factors and ribosomal proteins clustered based on their on their available cryo-EM data (PDB-6EM3, 6EM4, 6EM1, $6 E M 5,6 E L Z)^{15}$. Asterisk indicates the Nop2 bait protein. (C) Confirmation of mass spectrometry data through western blot analysis. Nop2-TAP particle were isolated as in $(A)$, and the indicated proteins were detected by western blotting using antibodies against endogenous UL3, eL15, the HA-tag for A3 factor $\mathrm{Cic} 1$, and the calmodulin binding protein (CBP) for Nop2. (D) Analysis of the requirement for catalytic activity in uL3 recruitment. Nop2TAP particles were isolated from $\Delta d b p 7$ strains harboring with empty pRS415 plasmid (EV) or

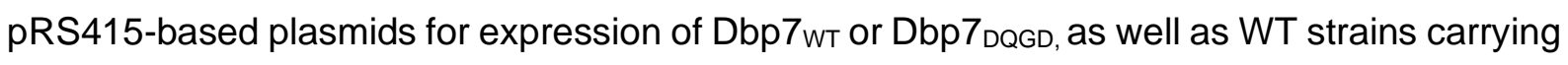
EV. Western blot analysis was performed as in (C). 


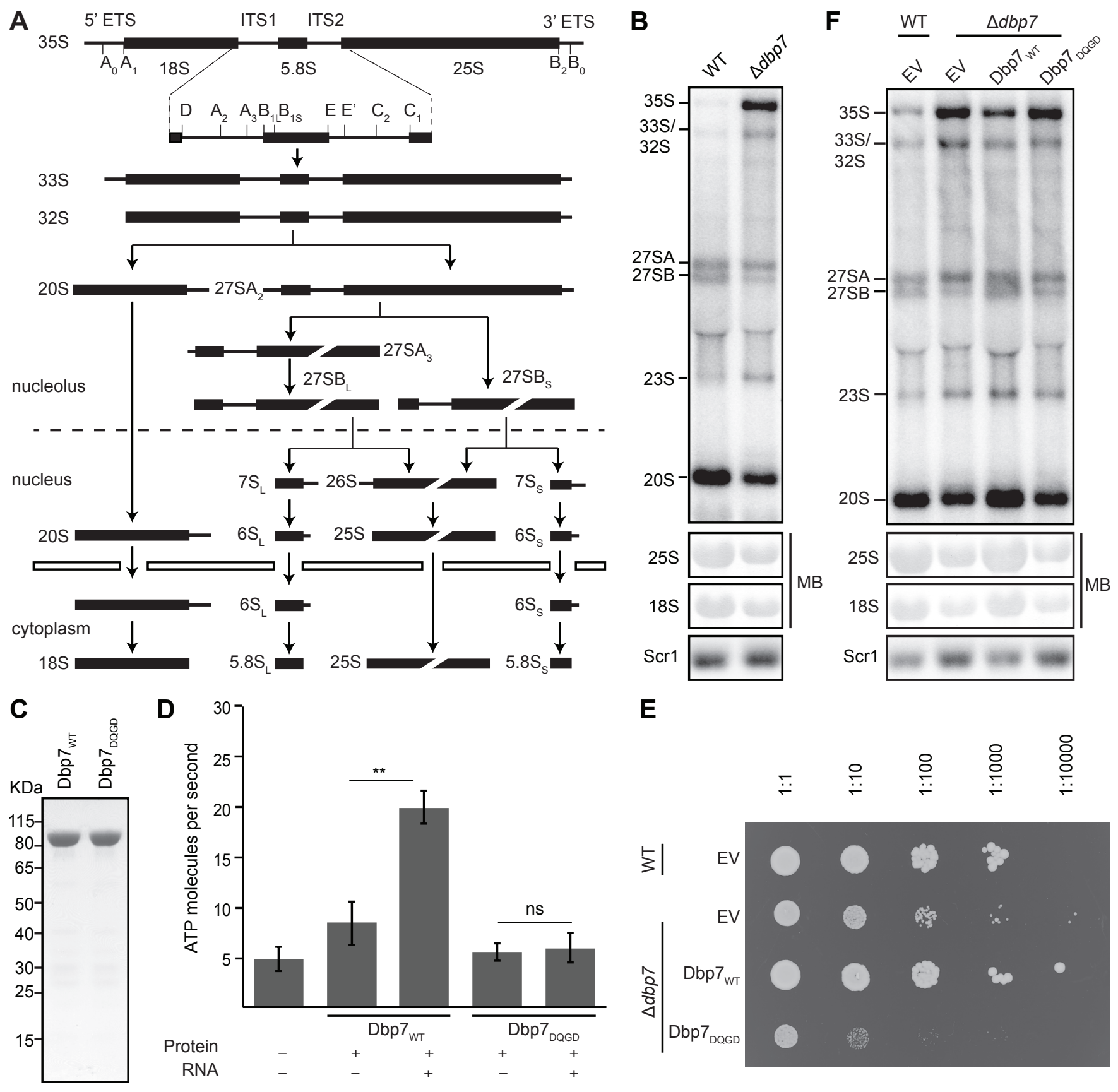

Aquino et al., Figure 1 

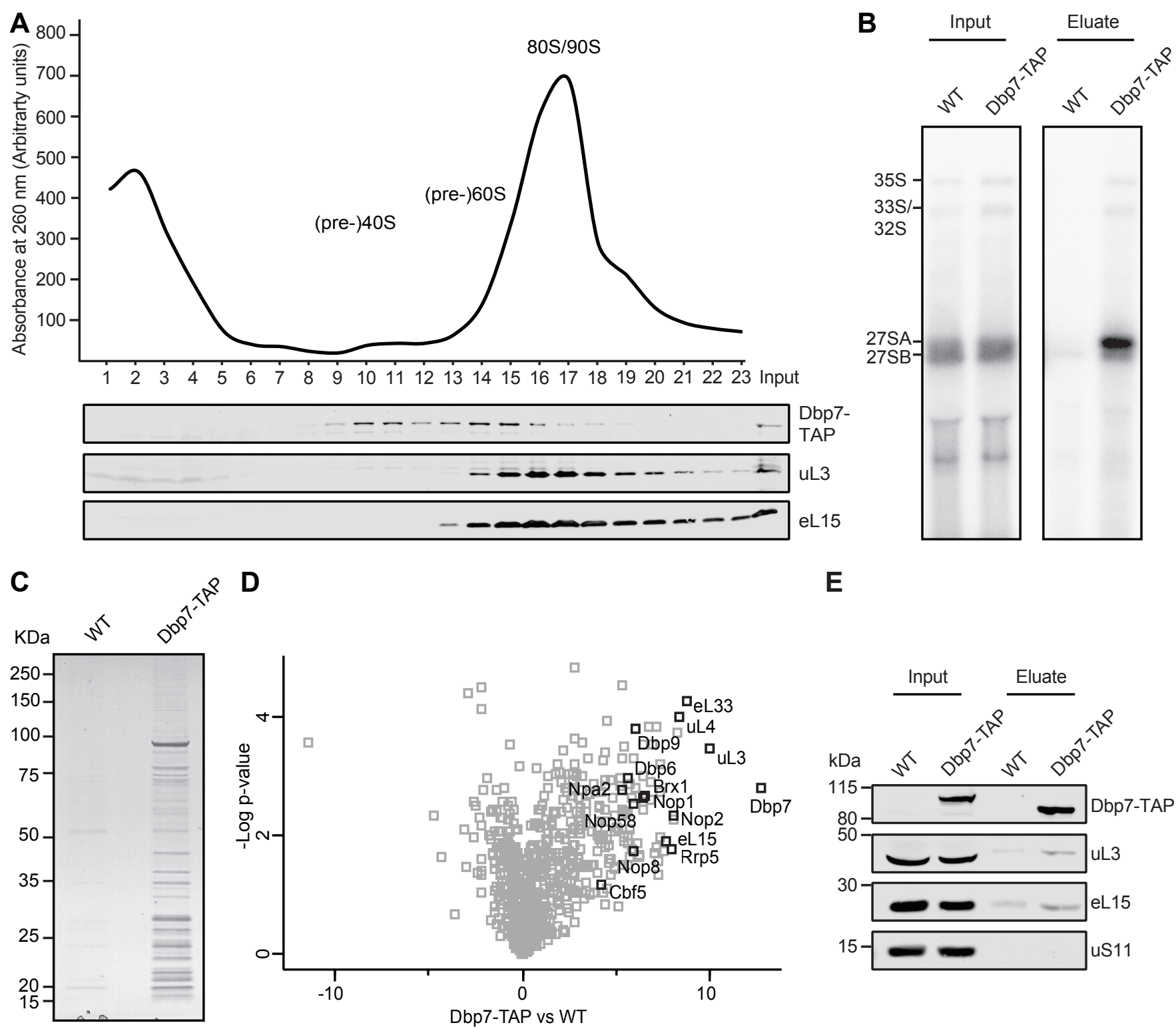

D

E
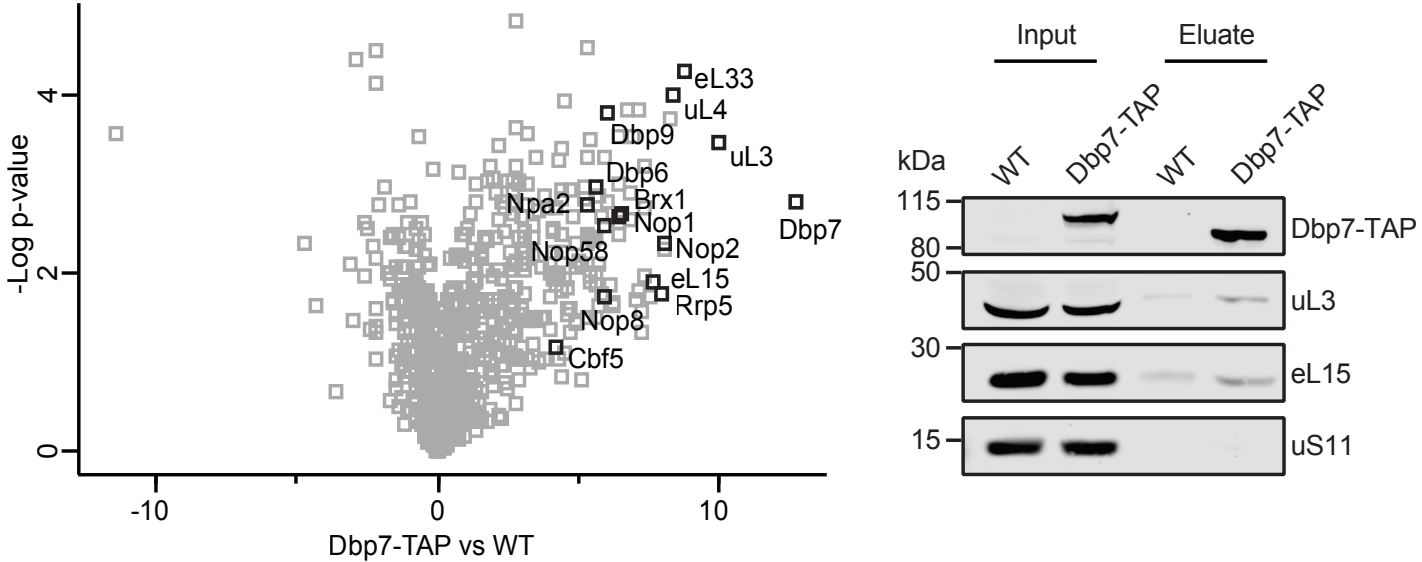

Aquino et al., Figure 2 
A

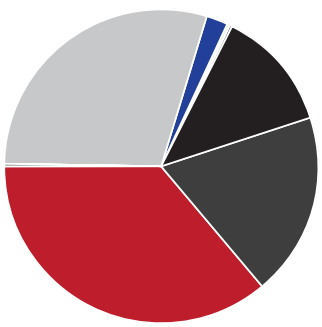

WT_1

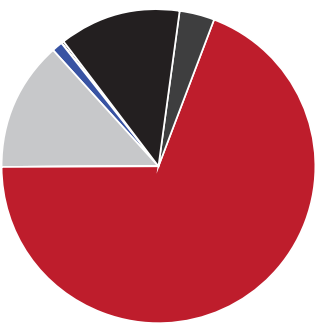

Dbp7-HTP_1

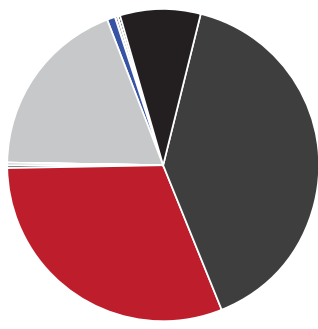

WT_2

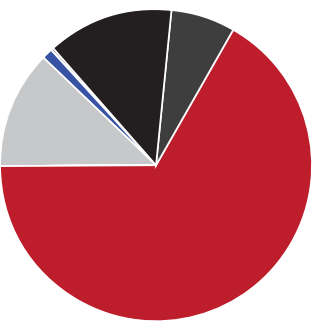

Dbp7-HTP_2
rRNA

protein coding

intergenic region

snoRNA

tRNA

other RNAs

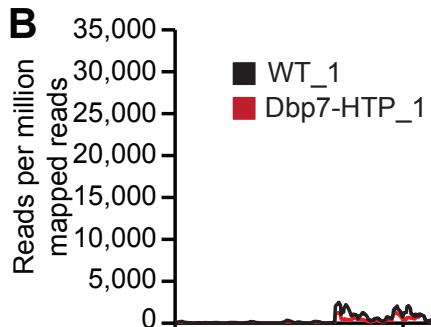

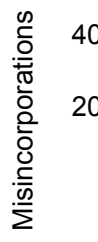
40,0007

Mh $A^{\downarrow}$

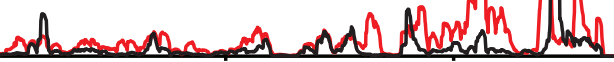
$0,000-$

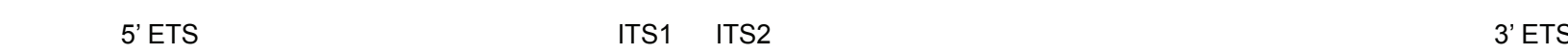
$35 S$

$18 S$

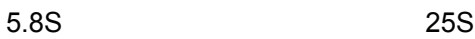

C

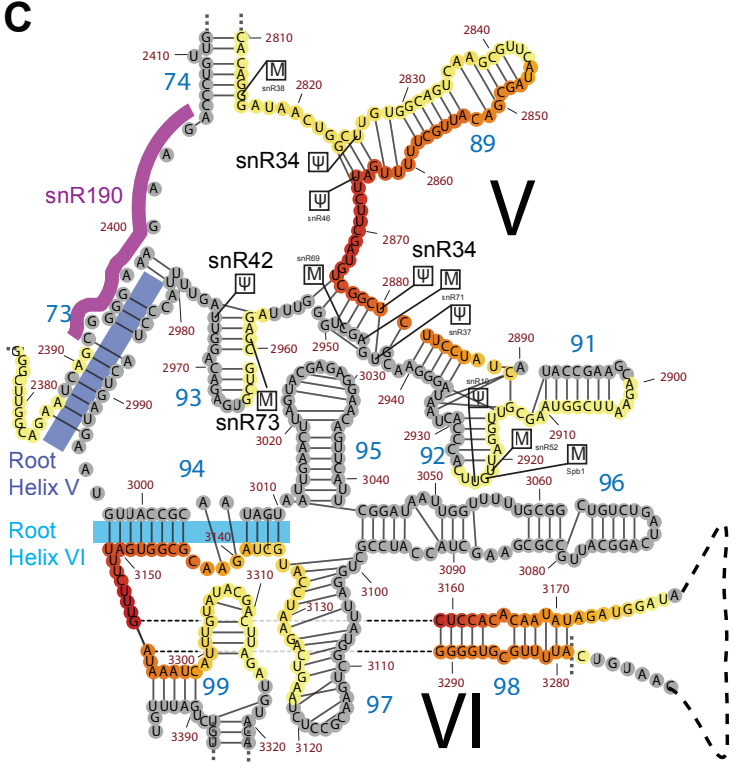

D

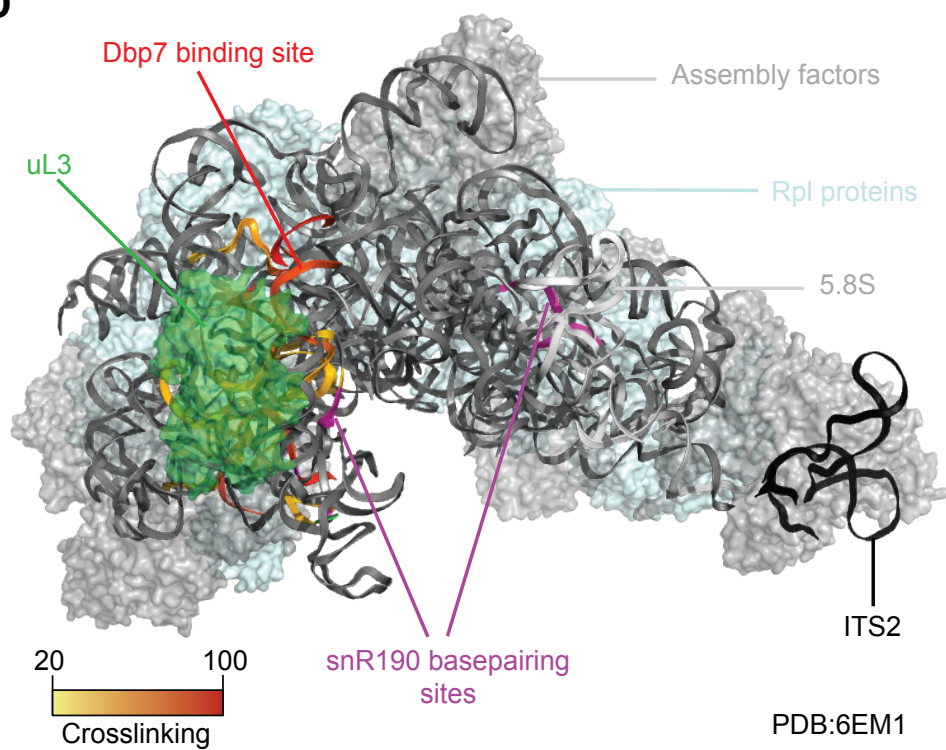

Aquino et al., Figure 3 

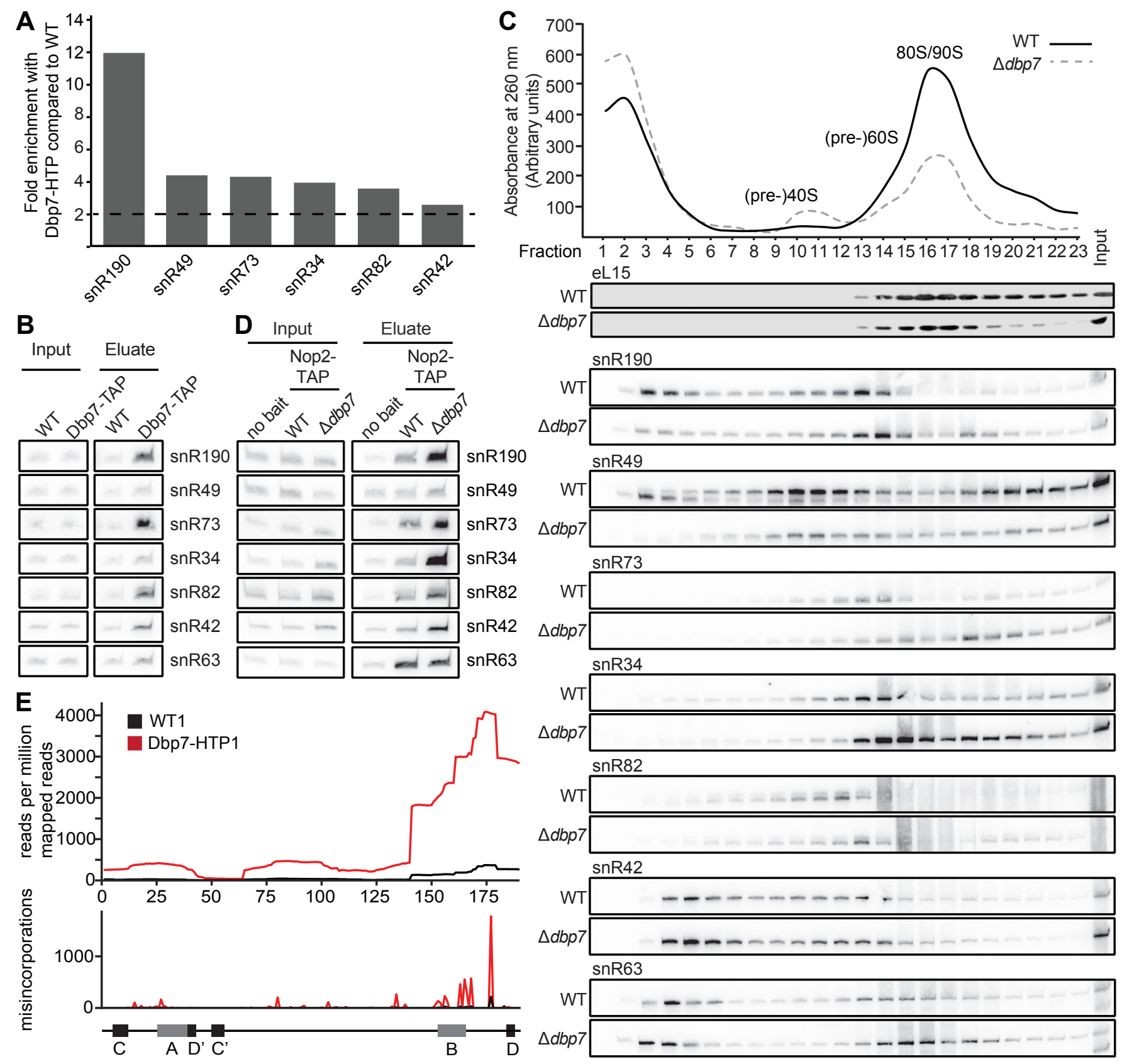

\section{Aquino et al., Figure 4}



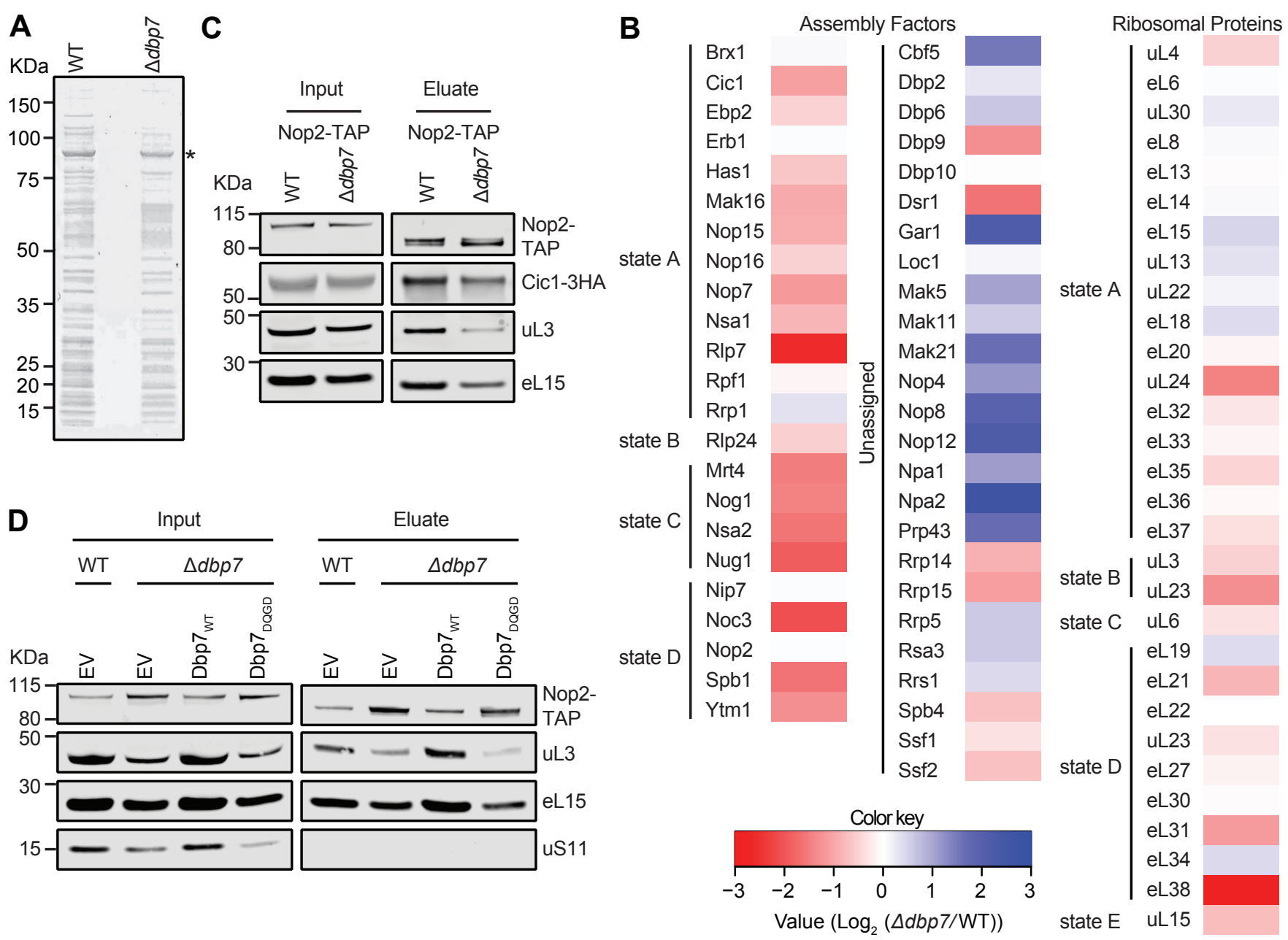

Aquino et al., Figure 5 


\section{SUPPLEMENTARY MATERIAL}

The RNA helicase Dbp7 promotes domain V/VI compaction and stabilization of interdomain interactions during early 605 assembly

Gerald Ryan R. Aquino, Philipp Hackert, Nicolai Krogh, Kuan-Ting Pan, Henrik Nielsen, Henning Urlaub, Katherine E. Bohnsack and Markus T. Bohnsack

\section{SUPPLEMENTARY FIGURES}

\section{Supplementary Figure S1. Dbp7 is a nucleolar RNA helicase required for normal growth} and pre-rRNA processing. (A) Quantification of the levels of pre-rRNA intermediates in Figure 1B. Signals were quantified using ImageStudioLite software and the amount of SRP RNA (Scr1) was used for normalization. Data is represented as mean \pm standard deviation from three independent experiments and significance was determined using Student's t-test $\left({ }^{*}=p<0.05\right.$, n.s. $=$ non-significant). (B) Fluorescence microscopy images showing cellular localization of Dbp7. Cell expressing Dbp7 with GFP tag were viewed under a fluorescence microscope. Nop1-RFP was used as nucleolar localization marker. Fluorescence microscopy images were overlaid with bright field images. Co-localization appears in yellow. Scale bare = 2 microns. (C) Growth analysis of WT and $\Delta d b p 7$. Absorbance at $600 \mathrm{~nm}$ were measured every 90 min for 9 hours of exponentially growing cells. Data are represented as mean \pm standard deviation from three independent experiments. (D) Expression level of Dbp7 from GALs promoter. Total protein was extracted from wild-type yeast (WT), cells expressing Dbp7-3HA from the endogenous $D B P 7$ promoter and cells expressing $3 \mathrm{HA}-\mathrm{Dbp} 7$ from a GALs promoter and protein levels were analysed by western blotting using antibodies against the HA-tag and Pgk1. Asterisk indicates a non-specific interaction of the anti-HA antibody. (E) Pre-rRNA processing in wild-type yeast (WT) and the pGALs-3HA-Dbp7 strain grown in YP medium containing galactose (Gal) or glucose (Glu) after $12 \mathrm{~h}$ was analysed by northern blotting similar to Figure 1B. (F-G) pGal ${ }_{s}$-mediated depletion of Dbp7 and its effect on growth. pGAL $L_{s}-3 H A-$ Dbp7 strains were grown exponentially for 12 hours in YP medium containing galactose or glucose, and Dbp7 expression (F) and growth $(G)$ were monitored every two hours. Dbp7 expression was analysed as in $D$ and the asterisk indicates a non-specific interaction of the anti-HA antibody. Growth was measured as in C. (H-I) Quantification of the levels of pre-rRNA intermediates in Figure S1E $(\mathrm{H})$ and Figure $1 \mathrm{~F}(\mathrm{I})$ as in $(\mathrm{A})$ and normalized according to the amount of scR1. Data is represented as mean \pm standard deviation from three independent experiments and significance was determined using student T-test $\left({ }^{*}=p<0.05,{ }^{* *}=p<0.01\right.$, ${ }^{* * *}=\mathrm{p}<0.001$ n.s. $=$ non-significant).

Supplementary Figure S2. Dbp7 is required for 2'-O-methylation of specific rRNA nucleotides. (A) RiboMeth-seq analysis of $\Delta d b p 7$ complementation system. Total RNA were 
extracted from exponentially growing cells and were then used for RMS analysis. RMS score was plotted for each 2'-O-methylated nucleotide in the 25S and 18S rRNAs. (B) RMS scores of each of the four strains at sites where there is significant variation were plotted as bar graph. Data are presented as mean \pm standard deviation from three independent measurements. (C) Mapping of Dbp7-dependent modifications on structure of early pre-60S particle. Affected sites were mapped in the so-called Nog2 pre-60S particle purified via TAP-tag ${ }^{36}$.

Supplementary Figure S3. Nop2-containing particles harbor Dbp7 and 27SB pre-rRNAs. (A) Dbp7 is present in pre-ribosomes purified via Nop2-TAP and Npa1-TAP. Nop2- or Npa2containing pre-60S particles were purified via TAP-tagged from cells expressing 3xHA-dbp7 in wild-type background strains (no bait) or in strains also expressing the bait protein (Nop2 or Npa2). Input (0.5\%) and eluate were separated in denaturing PAGE followed by western blotting. The presence of $\mathrm{Dbp} 7$ and bait proteins were detected using antibodies against HAand CBP-tag respectively while uL3, uL15, and uS11 were detected with antibodies against the endogenous proteins. (B) Nop2-contaning pre-60S particles from WT and $\Delta d b p 7$ cells were purified as in $(A)$ and RNAs in input $(0.2 \%)$ and eluate were extracted. Pre-rRNA species were detected by northern blotting as in Figure 1B.

Supplementary Figure S4. Structural analysis of ITS2 region via DMS structure probing. (A) Secondary structure of the hair-pin loop model of ITS2. Highlighted in blue, yellow and pink are the binding site of assembly factors Cic1, Nop15, and Npa1. The regions analysed in (B) are indicated by grey lines. (B) DMS structure probing of ITS2 region. Nop2-containing particles from WT and $\Delta d b p 7$ cells were captured via TAP tag and treated with DMS (+) or left untreated (-). RNAs associated with the complex were extracted and used as template for primer extension using radiolabelled probes (primer 1 and primer 2). cDNA transcripts were separated in a PAGE gel in parallel with sequencing ladder. Signals were visualized using a phosphoimager.

\section{SUPPLEMENTARY TABLES}

Supplementary Table 1. Ribosomal proteins and biogenesis factors enriched with Dbp7TAP

\begin{tabular}{|l|l|}
\hline Ribosomal Protein & $\begin{array}{l}\text { Fold-change (WT vs } \Delta \text { dbp7; } \\
\text { difference of } \text { Log }_{2} \text { ) }\end{array}$ \\
\hline uL3 & 10 \\
\hline eL19 & 8.5 \\
\hline uL4 & 8.3 \\
\hline eL33 & 8.1 \\
\hline uL25 & 7.9 \\
\hline eL39 & 7.6 \\
\hline uL30 & 7.4 \\
\hline
\end{tabular}




\begin{tabular}{|c|c|}
\hline uL1 & 7.3 \\
\hline eL34 & 7.2 \\
\hline uL13 & 7.2 \\
\hline eL15 & 7.1 \\
\hline eL13 & 6.9 \\
\hline eL24 & 6.6 \\
\hline uL10 & 6.6 \\
\hline eL8 & 6.5 \\
\hline P2 & 6.3 \\
\hline eL36 & 6.0 \\
\hline eL20 & 5.9 \\
\hline uL12 & 5.8 \\
\hline eL37 & 5.6 \\
\hline uL22 & 5.5 \\
\hline eL6 & 5.4 \\
\hline eL18 & 4.8 \\
\hline $\mathrm{P} 1$ & 4.8 \\
\hline eL22 & 4.4 \\
\hline uL9 & 4.1 \\
\hline $\mathrm{uL2}$ & 4.1 \\
\hline uL15 & 3.9 \\
\hline eL21 & 3.7 \\
\hline eL27 & 3.6 \\
\hline eL30 & 3.3 \\
\hline eL32 & 2.8 \\
\hline uL18 & 1.7 \\
\hline \multicolumn{2}{|c|}{ Assembly Factors } \\
\hline Dbp7 & 13.0 \\
\hline Puf6 & 8.0 \\
\hline Nop2 & 8.0 \\
\hline Nsa2 & 7.9 \\
\hline Rrp5 & 7.8 \\
\hline Nop1 & 6.8 \\
\hline Brx1 & 6.5 \\
\hline Urb1 & 6.5 \\
\hline Dbp9 & 6.4 \\
\hline Nop4 & 6.3 \\
\hline Mak5 & 5.8 \\
\hline Urb2 & 5.8 \\
\hline Nop8 & 5.7 \\
\hline Has1 & 5.7 \\
\hline Rrp12 & 5.6 \\
\hline Ebp2 & 5.5 \\
\hline Dbp6 & 5.3 \\
\hline Nip7 & 5.2 \\
\hline Rlp7 & 5.2 \\
\hline Nop58 & 5.1 \\
\hline Nog1 & 5.1 \\
\hline Nsa1 & 5.0 \\
\hline Mak21 & 4.9 \\
\hline Nog2 & 4.8 \\
\hline Ssf1 & 4.7 \\
\hline Cbf5 & 4.7 \\
\hline Erb1 & 4.6 \\
\hline
\end{tabular}




\begin{tabular}{|l|l|}
\hline Mrt4 & 4.3 \\
\hline Rok1 & 4.1 \\
\hline Nop7 & 3.9 \\
\hline Ytm1 & 3.8 \\
\hline Rpf1 & 3.8 \\
\hline Dbp10 & 3.7 \\
\hline Rrs1 & 3.6 \\
\hline Loc1 & 3.6 \\
\hline Cic1 & 3.4 \\
\hline Rsa3 & 3.3 \\
\hline Rrp14 & 3.2 \\
\hline Mdn1 & 3.2 \\
\hline Noc2 & 3.2 \\
\hline Mak11 & 3.2 \\
\hline Noc3 & 3.1 \\
\hline Dbp3 & 3.1 \\
\hline Drs1 & 3.0 \\
\hline Mak16 & 2.9 \\
\hline Mex67 & 2.9 \\
\hline Spb1 & 2.7 \\
\hline Xrn1 & 2.7 \\
\hline Sda1 & 2.3 \\
\hline Nop53 & 2.3 \\
\hline Prp43 & 1.9 \\
\hline Nug1 & 1.9 \\
\hline
\end{tabular}

Supplementary Table 2. snoRNA enrichment in with Dbp7

\begin{tabular}{|l|l|l|}
\hline snoRNA & Fold-enrichment (Dbp7-HTP_1) & Fold-enrichment (Dbp7-HTP_2) \\
\hline snR190 & 12 & 2.4 \\
\hline snR49 & 4.4 & 1.0 \\
\hline snR73 & 4.2 & 0.5 \\
\hline snR34 & 4.0 & 1.2 \\
\hline snR82 & 3.6 & 4.8 \\
\hline snR42 & 2.6 & 0.4 \\
\hline snR80 & 2.5 & 3.0 \\
\hline snR44 & 2.4 & 0.6 \\
\hline snR31 & 2.2 & 0.3 \\
\hline
\end{tabular}

Supplementary Table 3. Changes in protein composition of pre-60S particle depending on Dbp7

\begin{tabular}{|l|l|}
\hline $\begin{array}{l}\text { Ribosomal } \\
\text { Protein }\end{array}$ & $\begin{array}{l}\text { Fold-change (WT vs } \\
\text { Log }_{2} \text { of fold-change) }\end{array}$ \\
\hline uL3 & -0.5 \\
\hline uL4 & -0.5 \\
\hline eL6 & 0.1 \\
\hline uL30 & 0.3 \\
\hline eL8 & 0.1 \\
\hline uL6 & -0.3 \\
\hline eL14 & 0.1 \\
\hline eL15 & 0.5 \\
\hline uL13 & 0.3 \\
\hline
\end{tabular}




\begin{tabular}{|c|c|}
\hline uL22 & 0.2 \\
\hline eL18 & 0.4 \\
\hline eL19 & 0.4 \\
\hline eL20 & -0.1 \\
\hline eL21 & -0.8 \\
\hline eL22 & 0 \\
\hline uL14 & -1.3 \\
\hline uL23 & -0.3 \\
\hline uL24 & -1.5 \\
\hline eL27 & -0.2 \\
\hline uL15 & -0.8 \\
\hline eL30 & 0 \\
\hline eL31 & -1.2 \\
\hline eL32 & -0.3 \\
\hline eL33 & -0.1 \\
\hline eL34 & 0.4 \\
\hline uL29 & -0.5 \\
\hline eL36 & -0.1 \\
\hline eL37 & -0.4 \\
\hline eL38 & -3.0 \\
\hline \multicolumn{2}{|c|}{ Assembly Factors } \\
\hline Brx1 & 0.1 \\
\hline Cbf5 & 1.54 \\
\hline Cic1 & -1.1 \\
\hline Dbp10 & 0.0 \\
\hline Dbp2 & 0.2 \\
\hline Dbp6 & 0.6 \\
\hline Dbp9 & -1.3 \\
\hline Drs1 & -1.6 \\
\hline Ebp2 & -0.5 \\
\hline Erb1 & 0.1 \\
\hline Gar1 & 2.16 \\
\hline Has1 & -0.6 \\
\hline Loc1 & 0.1 \\
\hline Mak5 & 1.1 \\
\hline Mak11 & 1.6 \\
\hline Mak16 & -1.0 \\
\hline Mak21 & 0.6 \\
\hline Mrt4 & -1.5 \\
\hline Nip7 & 0.1 \\
\hline Noc3 & -2.1 \\
\hline Nog1 & -1.5 \\
\hline Nop2 & 0.0 \\
\hline Nop4 & 1.16 \\
\hline Nop7 & -1.2 \\
\hline Nop8 & 2.0 \\
\hline Nop12 & 2.2 \\
\hline Nop15 & -1.0 \\
\hline Nop16 & -0.5 \\
\hline Npa1 & 1.1 \\
\hline Npa2 & 2.7 \\
\hline Nsa1 & -0.8 \\
\hline Nsa2 & -1.6 \\
\hline Nug1 & -1.9 \\
\hline
\end{tabular}




\begin{tabular}{|l|l|}
\hline Prp43 & 1.7 \\
\hline Rlp24 & -0.5 \\
\hline Rlp7 & -2.6 \\
\hline Rpf1 & -0.1 \\
\hline Rrp1 & 0.3 \\
\hline Rrp5 & 0.6 \\
\hline Rrp14 & -0.8 \\
\hline Rrp15 & -1.1 \\
\hline Rrs1 & 0.6 \\
\hline Rsa3 & 0.4 \\
\hline Spb1 & -1.6 \\
\hline Spb4 & -0.7 \\
\hline Ssf1 & -0.7 \\
\hline Ssf2 & -0.3 \\
\hline Ytm1 & -1.3 \\
\hline
\end{tabular}

\section{Supplementary Table 4. DNA oligonucleotides used in this study}

\begin{tabular}{|c|c|c|}
\hline Name & Sequence $\left(5^{\prime}-3^{\prime}\right)$ & Application \\
\hline oMB1462 & TGAGAAGGAAATGACGCT & $\begin{array}{l}\text { Northern blot probe for } \\
\text { ITS1 }\end{array}$ \\
\hline oMB1468 & TGAGAAGGAAATGACGCT & $\begin{array}{l}\text { Northern blot probe for } \\
\text { ITS2 }\end{array}$ \\
\hline oMB2213 & GTCACAGGCGAAATATCATCAAAGTTAATC & $\begin{array}{l}\text { Northern blot probe for } \\
\text { snR73 }\end{array}$ \\
\hline oMB2543 & ATCCCGGCCGCCTCCATCAC & $\begin{array}{l}\text { Northern blot probe for } \\
\text { Scr1 }\end{array}$ \\
\hline oMB6604 & $\begin{array}{l}\text { atatatAGATCTGCTATAGCGCTGTTTGTTCCGT } \\
\text { TCTC }\end{array}$ & $\begin{array}{l}\text { Molecular cloning for } \\
\text { expression in yeast } \\
\text { (DBP7wT 500updown) }\end{array}$ \\
\hline oMB6605 & atatatGCGGCCGCCGTTGTTAGCATGAATATAG & $\begin{array}{l}\text { Molecular cloning for } \\
\text { expression in yeast } \\
\text { (DBP7wT_500updown) }\end{array}$ \\
\hline oMB6610 & $\begin{array}{l}\text { CTAAGGTATATCGTATTGGATCAAGGAGATAA } \\
\text { GTTAATGGAATTGG }\end{array}$ & $\begin{array}{l}\text { Site-directed } \\
\text { mutagenesis } \\
\left.\text { (Dbp7 } 7_{\text {DQGD }}\right)\end{array}$ \\
\hline oMB6611 & $\begin{array}{l}\text { CCAATTCCATTAACTTATCTCCTTGATCCAATA } \\
\text { CGATATACCTTAG }\end{array}$ & $\begin{array}{l}\text { Site-directed } \\
\text { mutagenesis } \\
\text { (Dbp7 } 7 \text { DQGD) }\end{array}$ \\
\hline oMB6620 & $\begin{array}{l}\text { atatatAGATCTATGAGCGATGAAGATTCTATGC } \\
\text { TG }\end{array}$ & $\begin{array}{l}\text { Molecular cloning for } \\
\text { recombinant expression }\end{array}$ \\
\hline oMB6621 & $\begin{array}{l}\text { atatatGCTAGCGTAGTTAAACTCACTTGCTATTT } \\
\text { G }\end{array}$ & $\begin{array}{l}\text { Molecular cloning for } \\
\text { recombinant expression }\end{array}$ \\
\hline oMB6691 & $\begin{array}{l}\text { GTTTCGTATGGCTCGTATGGCAGAGAAGCAA } \\
\text { ATAGCAAGTGAGTTTAACTACCGGATCCCCG } \\
\text { GGTTAATTAA }\end{array}$ & $\begin{array}{l}\text { 3xHA genomic tagging } \\
\text { (Dbp7-Fw) }\end{array}$ \\
\hline oMB6692 & $\begin{array}{l}\text { CTTTGTCTTCATAGTATACAATTTTTTTTTATAT } \\
\text { GAATTAATGCTTGTTCTTGTCTATGAATTCGA } \\
\text { GCTCGTTTAAAC }\end{array}$ & $\begin{array}{l}\text { 3xHA genomic tagging } \\
\text { (Dbp7-Rev) }\end{array}$ \\
\hline oMB6750 & $\begin{array}{l}\text { TATTCATATTCCAAATAAGCATACTTATTCAGC } \\
\text { AATTTAACACCAAGATGCGTACGCTGCAGGTC } \\
\text { GAC }\end{array}$ & $\begin{array}{l}\text { pGALs-3xHA genomic } \\
\text { tagging (Dbp7-Fw) }\end{array}$ \\
\hline oMB6751 & $\begin{array}{l}\text { GCAGTGTCCTCATTGGTAGTGAAGTTTAACAG } \\
\text { CATAGAATCTTCATCGCTCATCGATGAATTCT } \\
\text { CTGTCG }\end{array}$ & $\begin{array}{l}\text { pGALs-3xHA genomic } \\
\text { tagging (Dbp7-Rev) }\end{array}$ \\
\hline
\end{tabular}




\begin{tabular}{|c|c|c|}
\hline oMB6888 & CGTGCGTCTGATTATGGTCC & $\begin{array}{l}\text { Northern blot probe for } \\
\text { snR63 }\end{array}$ \\
\hline oMB8024 & $\begin{array}{l}\text { CAATCTTTTGAAAAAGGGTGTCAATCCAAAAG } \\
\text { CTAAAAGACCTTCTAACGAAAAAATGGAAAAG } \\
\text { AGAAGATGGAAAAAGAATTTC }\end{array}$ & $\begin{array}{l}\text { TAP genomic tagging } \\
\text { (Nop2-Fw) }\end{array}$ \\
\hline oMB8025 & $\begin{array}{l}\text { AGATTAGAGAGAGAAAACTATGCTAACATGAT } \\
\text { GCCACTACGTTTGTGGGAACTACGACTCACTA } \\
\text { TAGGGCGAATTGGG }\end{array}$ & $\begin{array}{l}\text { TAP genomic tagging } \\
\text { (Nop2-Rev) }\end{array}$ \\
\hline oMB8028 & $\begin{array}{l}\text { ATATTTCAAAGCACTTTACCTCCAATACAAAAA } \\
\text { GGTTGGTAAATGGCGCGAAGATATGGAAAAG } \\
\text { AGAAGATGGAAAAAGAATTT }\end{array}$ & $\begin{array}{l}\text { TAP genomic tagging } \\
\text { (Urb2-Fw) }\end{array}$ \\
\hline oMB8029 & $\begin{array}{l}\text { TGTTATTAAACGTGAGCAGAGAAATGCCTTTT } \\
\text { GAAAACACACTAAAACACATAAGTACGACTCA } \\
\text { CTATAGGGCGAATTGGG }\end{array}$ & $\begin{array}{l}\text { TAP genomic tagging } \\
\text { (Urb2-Rev) }\end{array}$ \\
\hline oMB8097 & $\begin{array}{l}\text { GAAGACGATAAGTACATTGAAAAGTCTGTGAA } \\
\text { AAACAATCTTTTGAAAAAGGGTGTCAATCCAA } \\
\text { AAGC }\end{array}$ & $\begin{array}{l}\text { TAP genomic tagging } \\
\text { (Nop2-Fw2) }\end{array}$ \\
\hline oMB8098 & $\begin{array}{l}\text { TGATATATATATATATATATATATATGTAGACA } \\
\text { GAGAAAGATTAGAGAGAGAAAACTATGCTAAC }\end{array}$ & $\begin{array}{l}\text { TAP genomic tagging } \\
\text { (Nop2-Rev2) }\end{array}$ \\
\hline oMB8101 & $\begin{array}{l}\text { CAATTGAACGCTTTCCTTGACACACCTGGGAA } \\
\text { ACAATATTTCAAAGCACTTTACCTCCAATAC }\end{array}$ & $\begin{array}{l}\text { TAP genomic tagging } \\
\text { (Urb2-Fw) }\end{array}$ \\
\hline oMB8102 & $\begin{array}{l}\text { TTTATCAATTTTTACTTGTTTAAGCTCCGTCAC } \\
\text { CCTGTTATTAAACGTGAGCAGAGAAATGCC }\end{array}$ & $\begin{array}{l}\text { TAP genomic tagging } \\
\text { (Urb2-Rev) }\end{array}$ \\
\hline oMB8426 & CGCTCTCTTCTTATCGATAACG & $\begin{array}{l}\text { DMS structure probing } \\
\text { of ITS2 }\end{array}$ \\
\hline oMB8427 & GCACGCAGAGAAACCTCTCTTTGG & $\begin{array}{l}\text { DMS structure probing } \\
\text { of ITS2 }\end{array}$ \\
\hline oMB8945 & CCTTGTCGTCATGGTCGAATCG & $\begin{array}{l}\text { Northern blot probe for } \\
\text { snR190 }\end{array}$ \\
\hline oMB8946 & CTCCCGGTAACCAGGCCAGCAG & $\begin{array}{l}\text { Northern blot probe for } \\
\text { snR82 }\end{array}$ \\
\hline oMB8985 & CGGACTTCCTATCCTGTCC & $\begin{array}{l}\text { Northern blot probe for } \\
\text { snR34 }\end{array}$ \\
\hline oMB8986 & GCAACCTCTAACGGCGATGG & $\begin{array}{l}\text { Northern blot probe for } \\
\text { snR42 }\end{array}$ \\
\hline oMB9023 & CGGGATTCGTTTACCATAGGCTACC & $\begin{array}{l}\text { Northern blot probe for } \\
\text { snR49 }\end{array}$ \\
\hline oMB9050 & $\begin{array}{l}\text { TAGCGAGCTTGAAAAAGAATCTAGCGAGTCA } \\
\text { GAAGCTGTCAAGAAGGCTAAAAGTCGTACGC } \\
\text { TGCAGGTCGAC }\end{array}$ & $\begin{array}{l}\text { GFP genomic tagging } \\
\text { (Dbp7-Fw) }\end{array}$ \\
\hline oMB9051 & $\begin{array}{l}\text { TTTTTCTTCACAAGAAAAAAATGAGAGAAAAG } \\
\text { ATAGATAAGGAGGAAACAAATTAATCGATGAA } \\
\text { TTCGAGCTCG }\end{array}$ & $\begin{array}{l}\text { GFP genomic tagging } \\
\text { (Dbp7-Rev) }\end{array}$ \\
\hline
\end{tabular}

\section{Supplementary Table 5. Constructs used in this study}

\begin{tabular}{|c|c|c|}
\hline Name & Description & Application \\
\hline pMB31 & pRS415 & $\begin{array}{l}\text { Protein expression in yeast } \\
\text { (complementation) }\end{array}$ \\
\hline pMB203 & $\begin{array}{l}\text { pFA6a-3xHA-His3MX6 (Longtine et } \\
\text { al., 1998) }\end{array}$ & Amplification of $3 \times \mathrm{HA}$ cassette \\
\hline pMB318 & pQE-80-nHis10-ZZ-TEV-Dbp7 & Recombinant protein expression \\
\hline
\end{tabular}




\begin{tabular}{|c|c|c|}
\hline pMB541 & pUN100 mRFP-Nop1 & Immunofluorescence microscopy \\
\hline pMB939 & pYM13 (Janke et al., 2004) & Amplification of TAP cassette \\
\hline pMB951 & pYM25 (Janke et al., 2004) & Amplification of GFP cassette \\
\hline pMB1005 & pYM-N32 (Janke et al., 2004) & $\begin{array}{l}\text { Amplification of pGALs-3HA } \\
\text { cassette }\end{array}$ \\
\hline pMB1364 & pRS415-DBP7wT_500updown & $\begin{array}{l}\text { Protein expression in yeast } \\
\text { (complementation) }\end{array}$ \\
\hline pMB1380 & pQE-80-nHis10-ZZ-TEV-Dbp7DQGD & Recombinant protein expression \\
\hline pMB1381 & pRS415_DBP7DQGD_500updown & $\begin{array}{l}\text { Protein expression in yeast } \\
\text { (complementation) }\end{array}$ \\
\hline
\end{tabular}

Supplementary Table 6. Yeast strains used in this study

\begin{tabular}{|l|l|l|}
\hline Name & Genotype & Reference \\
\hline YMB006/BY4741a & MATa;his $\Delta 1 ;$ leu2 $\Delta 0 ;$ met15 $0 ;$ ura $\Delta 0$ & Euroscarf \\
\hline YMB489 & YMB006; DBP7-HisTAP (URA3) & D. Tollervey \\
\hline YMB1470 & YMB006; $\Delta$ DBP7::kanMX6 & Euroscarf \\
\hline YMB1487 & YMB006; pMB031 (LEU2) & This study \\
\hline YMB1488 & YMB1470; pMB031 (LEU2) & This study \\
\hline YMB1489 & YMB1470; pMB1364 (LEU2) & This study \\
\hline YMB1490 & YMB1470; pMB1381 (LEU2) & This study \\
\hline YMB1533 & YMB006; DBP7-3xHA (HIS3) & This study \\
\hline YMB1541 & YMB006; pGALs-3xHA-DBP7 (natNT2) & This study \\
\hline YMB1693 & YMB006; DBP7-TAP (kanMX6) & This study \\
\hline YMB1819 & YMB006; NOP2-TAP (URA3) & This study \\
\hline YMB1825 & YMB1541; NOP2-TAP (URA3) & This study \\
\hline YMB1829 & YMB1541; URB2-TAP (URA3) & This study \\
\hline YMB1831 & YMB1470; NOP2-TAP (URA3) & This study \\
\hline YMB1909 & YMB1819; CIC1-3xHA: (hphNT1) & This study \\
\hline YMB1920 & YMB1470; CIC1-3xHA (hphNT1) & This study \\
\hline YMB1945 & YMB1819; pMB031 (LEU2) & This study \\
\hline YMB1946 & YMB1831; pMB031 (LEU2) & This study \\
\hline YMB1947 & YMB1831; pMB1364 (LEU2) & This study \\
\hline YMB1948 & YMB1831; pMB1381 (LEU2) & This study \\
\hline YMB1951 & YMB006; DBP7-yeGFP (hphNT1) & This study \\
\hline YMB1954 & YMB1951; pMB541 (LEU2) & This study \\
\hline
\end{tabular}

Supplementary Table 7. Antibodies used in this study

\begin{tabular}{|l|l|}
\hline Name & Source \\
\hline Anti-HA & Sigma cat \# H3663 \\
\hline Anti-Pgk1 & ThermoFisher Scientific cat \# 459250 \\
\hline Anti-PAP & Sigma cat \# P1291 \\
\hline Anti-CBP & Antibodies-online cat\# ABIN3181196 \\
\hline Anti-Rpl3 & Mybio-source cat\# MBS9214187 \\
\hline Anti-Rpl15 & Aviva systems bio cat\# ARP65141_P050 \\
\hline Anti-Rps14 & Aviva systems bio cat\#ARP40322_T100 \\
\hline
\end{tabular}




\section{SUPPLEMENTARY METHODS}

\section{RiboMeth-seq}

Yeast strains were grown exponentially to an $\mathrm{OD}_{600}$ of 0.8 . Total RNA was extracted and $5 \mu \mathrm{g}$ was used for RiboMeth-seq seq ${ }^{34}$. In brief, $5 \mu \mathrm{g}$ total RNA was fragmented under denaturing conditions in alkaline buffer ( $\mathrm{pH}$ 9.9). Then, the RNA was separated on a denaturing (urea) polyacrylamide gel, fragments in the size range 20-40 nt were excised and ligated to adapters using a modified tRNA ligase. cDNA was made using Superscript III (Thermo Fisher Scientific) and sequenced on a PI Chip v3 using the Ion Proton platform. Reads were mapped to the yeast transcriptome, and the RMS score in rRNAs (fraction methylated) calculated as "score C" as previously described ${ }^{34}$. In a few cases, a barcode correction was applied when calculating the RMS score as described previously ${ }^{35}$. The numbers of sequencing reads mapping to specific RNAs was used as a measure of their levels and is presented as reads per kilobase of transcript, per million mapped reads (RPKM) \pm SEM. Statistical significance was determined using Student's t-test $(p<0.05)$.

\section{DMS chemical probing}

Dimethyl sulphate (DMS) structure probing was carried out as previously described 27,40,76. Briefly, Nop2-containing particles were purified total cell lysate using TAP tag and were treated or not treated with DMS (final concentration $0.5 \%$ ) for 2 min followed by quenching with addition of $250 \mathrm{mM} \beta$-mercaptoethanol. Treated or not treated, co-precipitated RNAs were extracted and used as template for primer extension reaction using 5'-[32P]-labelled DNA oligonucleotides (5'-CGCTCTCTTCTTATCGATAACG-3' and 5'GCACGCAGAGAAACCTCTCTTTGG-3') and SuperScript III reverse transcriptase. cDNA fragments were separated in 10\% denaturing PAGE (7M Urea) gel together with sequencing ladder generated from primer extension of total RNA in the presence of small amounts of dideoxynucleotides. Signals were visualised using phosphorimager.

\section{Fluorescence Microscopy}

Exponentially growing cells in Synthetic media without Leucine were image at room temperature automatically with a Nikon Ti2 2-E inverted microscope. Focal plane was set using Perfect Focus System (Nikon). Images were acquired using an oil objective lens (Plan Apo Lambda 100x/1.45 oil) at 100x magnification using the appropriate filters for GFP (Ex 470/24, Em 520/35, Dichroic 488) or mRFP (Ex 395/25, Em433/24, Dichroic 405). 


\section{SUPPLEMENTARY REFERENCES}

27. Brüning, L. et al. RNA helicases mediate structural transitions and compositional changes in pre-ribosomal complexes. Nat. Commun. 9, 5383 (2018).

34. Birkedal, U. et al. Profiling of ribose methylations in RNA by high-throughput sequencing. Angew. Chem. Int. Ed Engl. 54, 451-455 (2015).

35. Krogh, N., Birkedal, U. \& Nielsen, H. RiboMeth-seq: Profiling of 2'-O-Me in RNA. Methods Mol. Biol. Clifton NJ 1562, 189-209 (2017).

40. Martin, R. et al. A pre-ribosomal RNA interaction network involving snoRNAs and the Rok1 helicase. RNA 20, 1173-1182 (2014).

76. Swiatkowska, A. et al. Kinetic analysis of pre-ribosome structure in vivo. RNA 18, 2187-2200 (2012). 

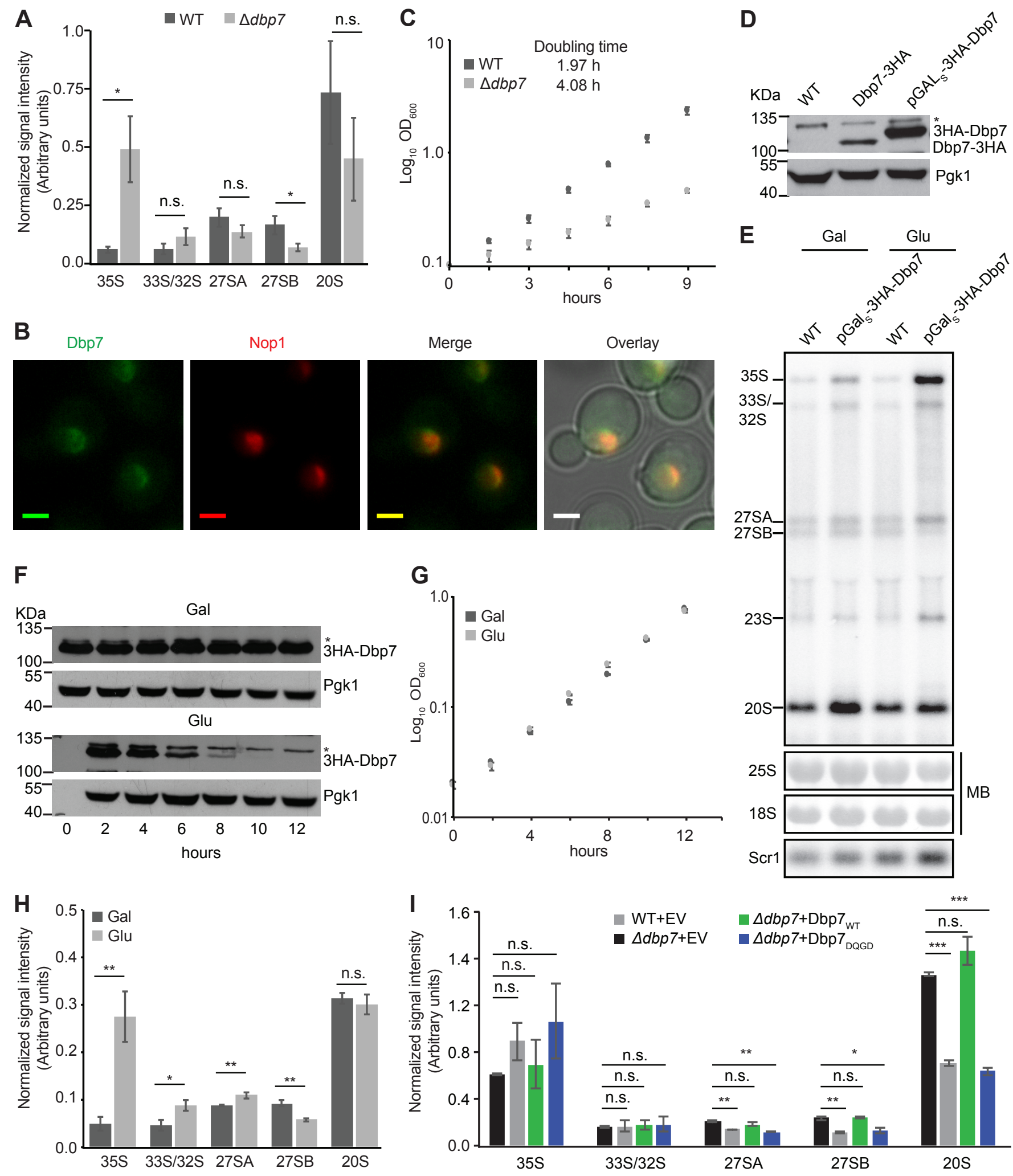

Aquino et al., Figure S1 

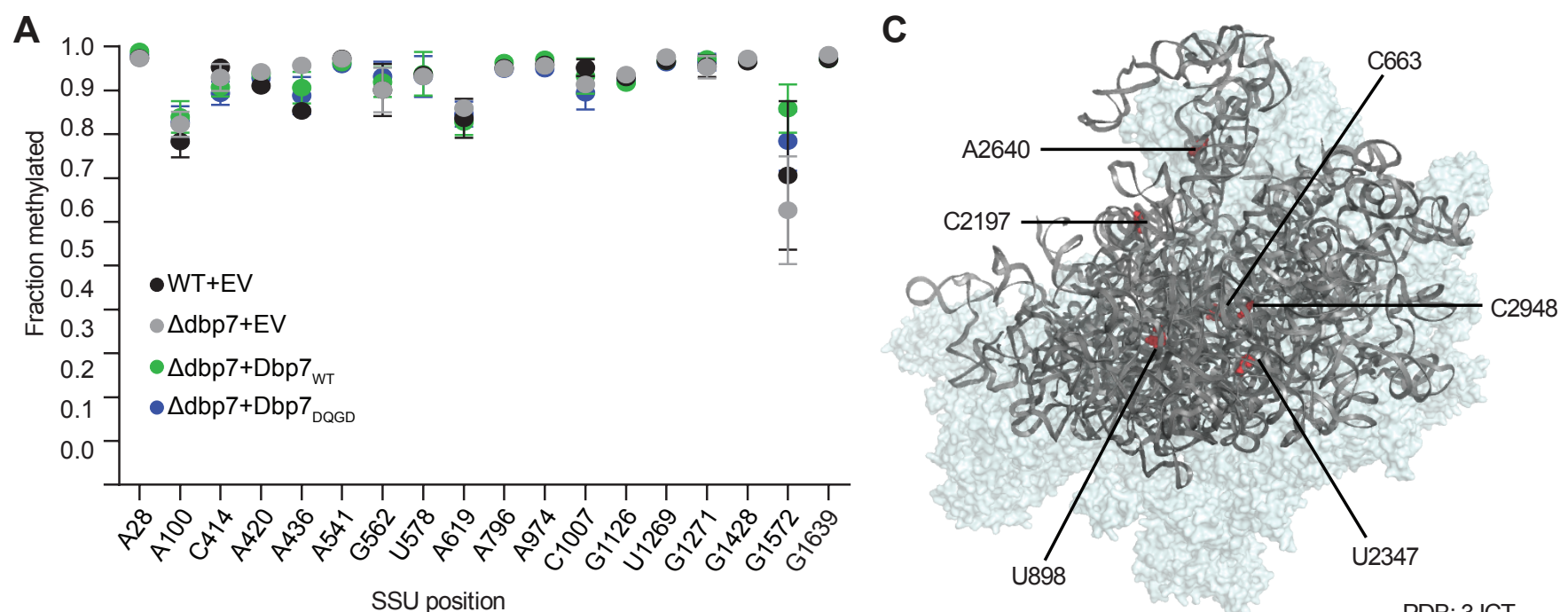

SSU position

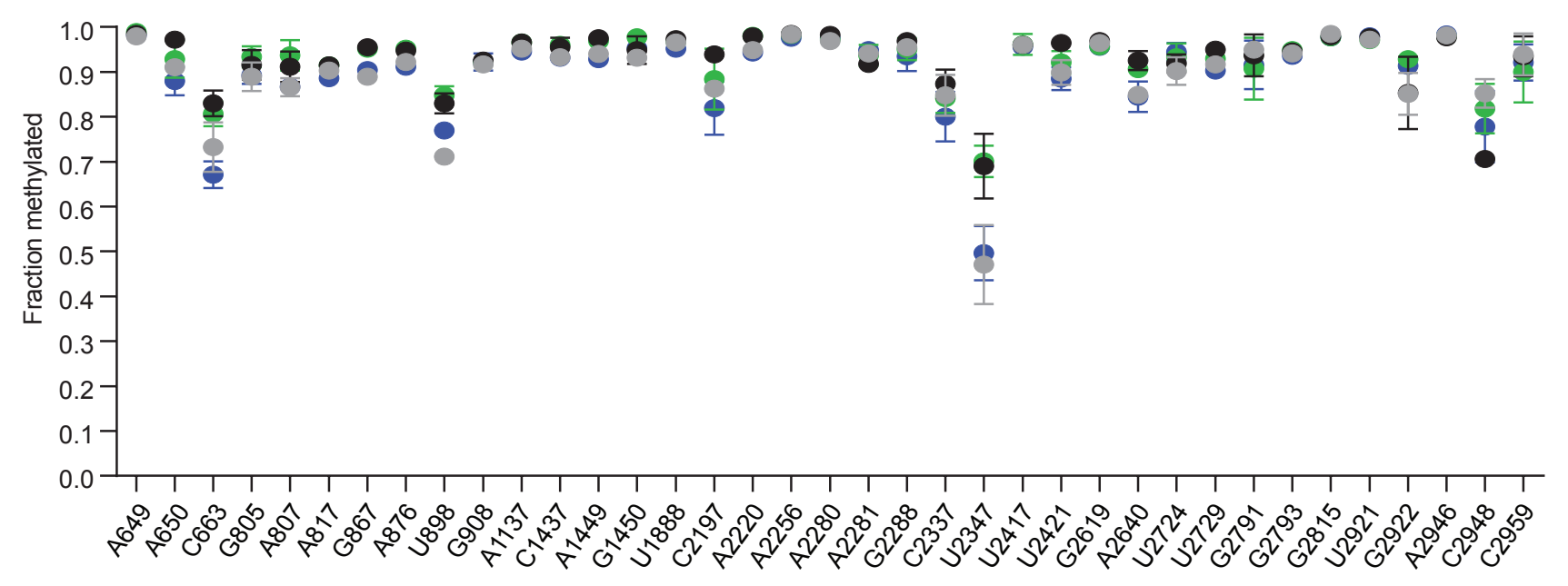

LSU position

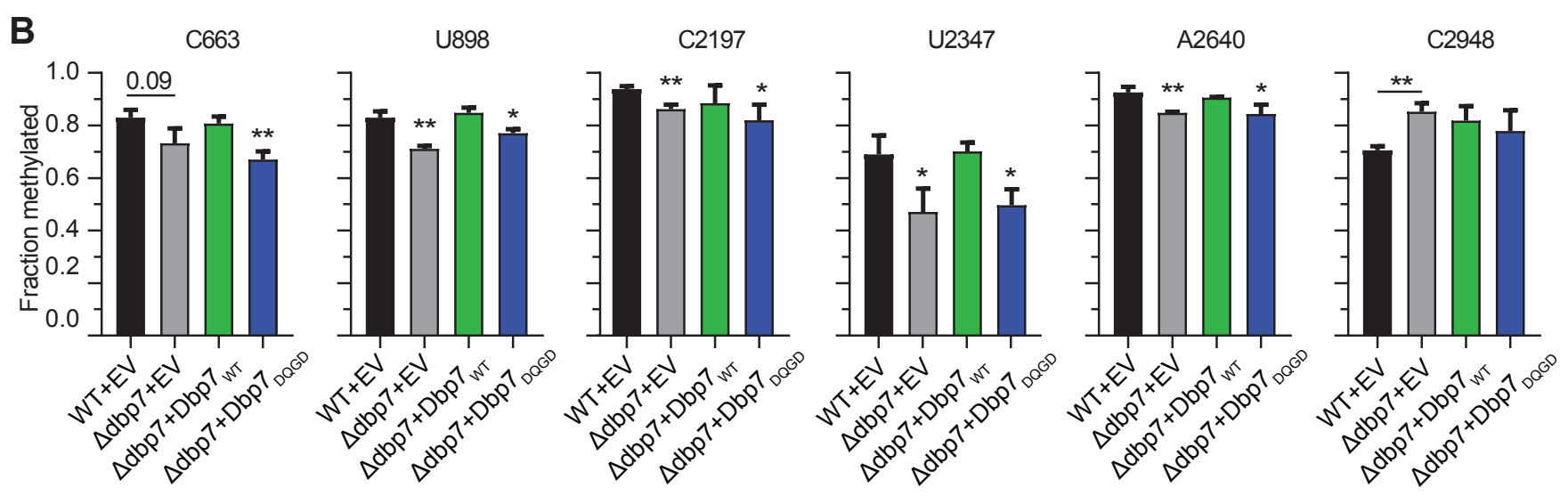

Aquino et al., Figure S2 

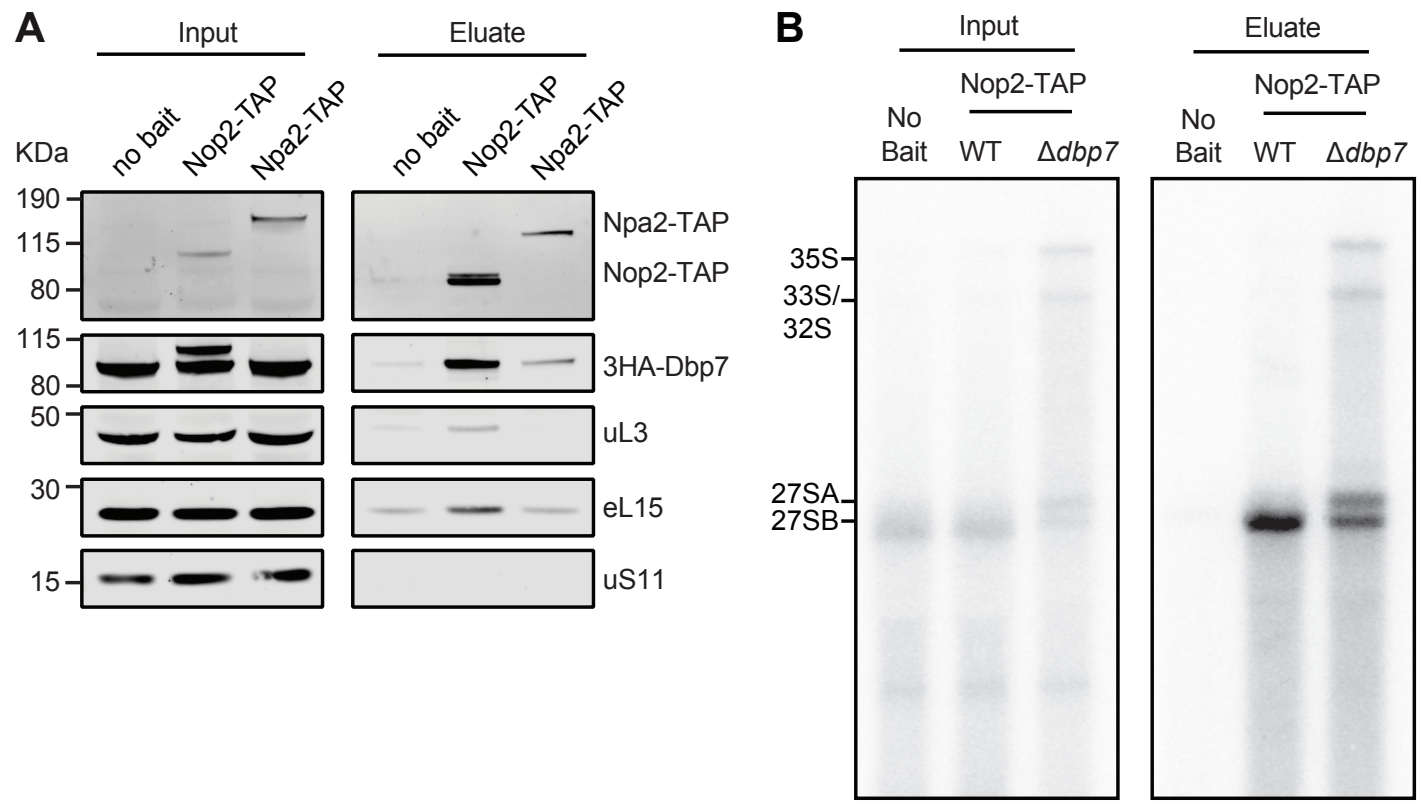

Aquino et al., Figure S3 
A

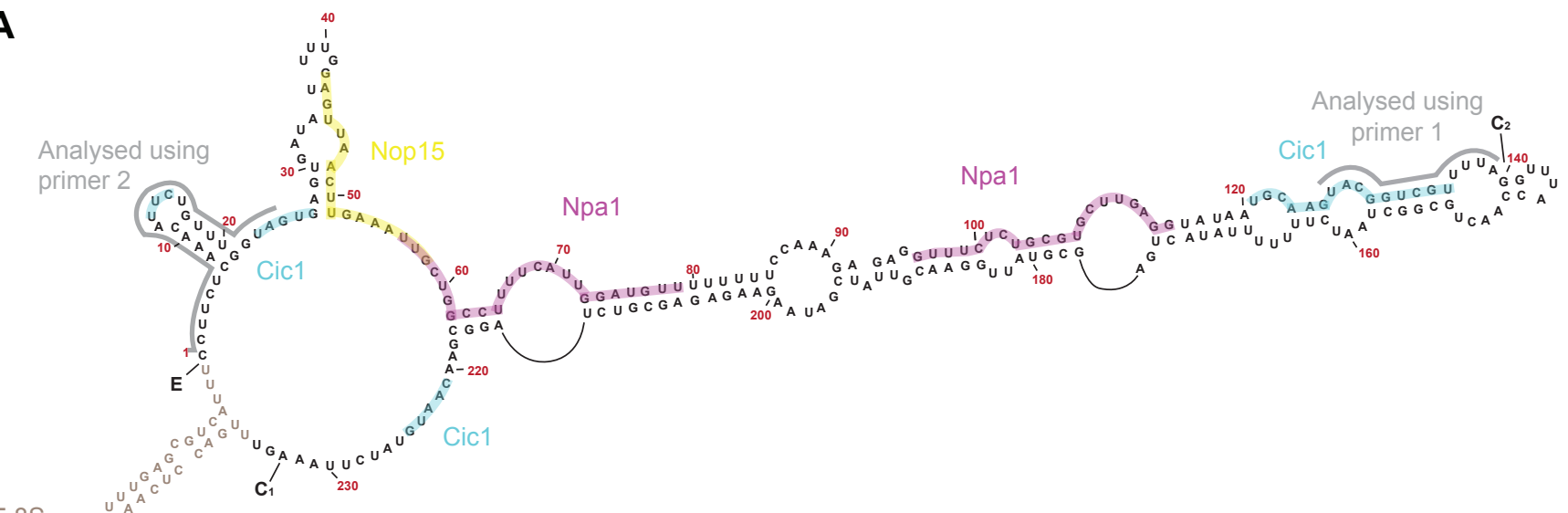

5.8S $U_{A}^{G}{ }^{G}{ }^{A}$ ITS2 proximal stem

${ }_{A}^{G}{ }^{G} 25 S$

B

A C G T $\quad \frac{\text { WT }}{-}+\frac{\Delta d b p 7}{+-}$ DMS $\quad$ A $\quad$ C $\quad$ G $\quad T \quad \frac{\text { W }}{-}+\frac{\Delta d b p 7}{+}$ DMS

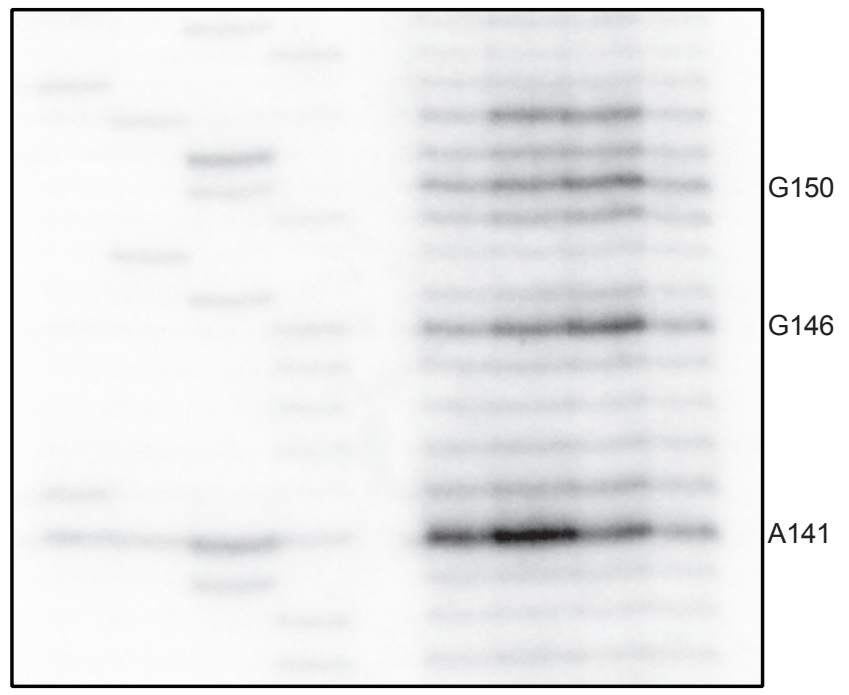

primer 1

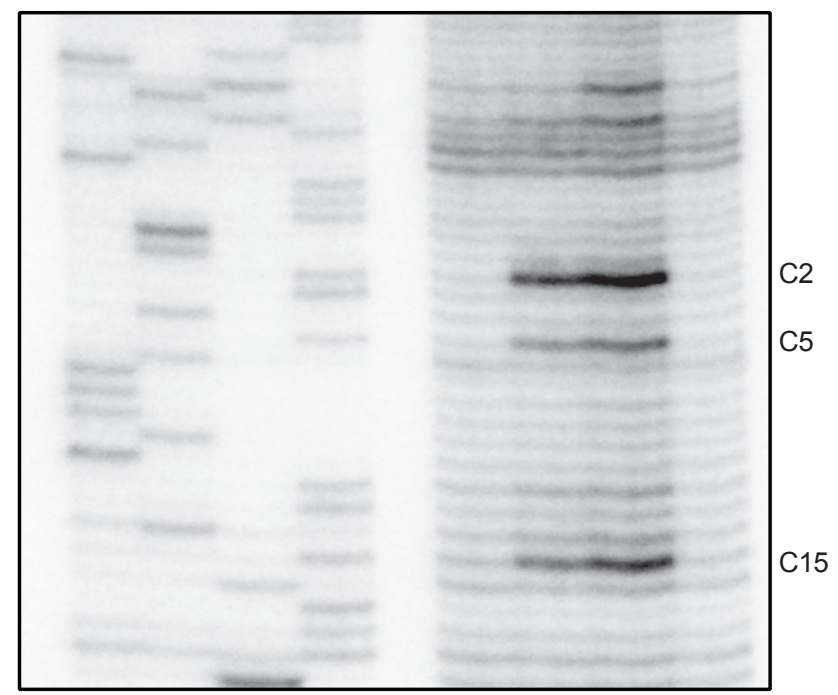

primer 2

Aquino et al., Figure S4 


\section{Chapter Four: Manuscript II}

Aquino GRR, Krogh N, Hackert P, Martin R, Davila Gallesio J, van Nues RW, Schneider C, Watkins NJ, Nielsen H, Bohnsack MT, Bohnsack KE. RNA helicase-mediated regulation of snoRNP dynamics on pre-ribosomes and rRNA 2'-O-methylation (2021). Nucleic Acid Res. In revision.

\section{Authors contributions}

Figure1: Protein expression and purification (PH); NADH-coupled ATPase Assay (PH)

Figure 2: Generation of yeast strains and northern blot analysis of pre-rRNA processing and quantification (RVN, NW, GRRA)

Figure 3: Preparation of sample for RiboMeth-seq (PH); RiboMeth-seq experiment (NK); RNase $\mathrm{H}$-based cleavage assay (GRRA)

Figure 4: Pulldown experiments and northern blotting (GRRA); RiboMeth-seq data analysis (see below); Analysis of snoRNAs distribution using sucrose gradient density centrifugation followed by northern blotting (GRRA)

Figure 5: Quantitative real-time PCR analysis of Box C/D snoRNAs distribution between free pool and pre-ribosomal fractions (RM)

Figure 6: Mapping of modification sites on rRNA secondary structure (KEB); northern blot analysis of snoRNAs distribution in free pool and pre-ribosomal fractions (GRRA)

Figure 7: Molecular cloning, generation of yeast strains, RNase $\mathrm{H}$-based cleavage assay (GRRA)

Figure 8: Generation of yeast strains and protein depletion assay (JDG); RiboMeth-seq experiment (NK); CLASH analysis (RM)

Figure S1: Generation of yeast strain (PH); RNase H-based cleavage assay (GRRA)

Figure S2: Quantification of snoRNAs expression (NK); Northern blot analysis of snoRNA expression levels (GRRA)

Figure S3: RiboMeth-seq data analysis (see below)

Figure S4: Quantitative real-time PCR analysis of Box H/ACA snoRNAs distribution between free pool and pre-ribosomal fractions (RM)

Figure S5: Mapping of modification sites in rRNA secondary structure (KEB)

Figure S6: Northern blot analysis of snR67 distribution in free pool and pre-ribosomal fractions (GRRA); Northern blot analysis of snR67 overexpression (GRRA)

Figure S7: Quantification of levels of snoRNAs expression upon Prp43 depletion (NK)

Non-experimental contribution: KEB and MTB conceived the study and supervised the work. NK, KEB, HN, MTB, CS and NJW analyzed rRNA 2'-O-methylation and pre-rRNA processing data. KEB and MTB wrote the manuscript. All authors analyzed and contributed to the preparation of manuscript. 


\section{RNA helicase-mediated regulation of snoRNP dynamics on pre- ribosomes and rRNA 2'-O-methylation}

Gerald Ryan R. Aquino1, Nicolai Krogh², Philipp Hackert ${ }^{1}$, Roman Martin ${ }^{1}$, Jimena Davila Gallesio ${ }^{1}$, Robert W. van Nues ${ }^{3}$, Claudia Schneider ${ }^{3}$, Nicholas J. Watkins ${ }^{3}$, Henrik Nielsen², Markus T. Bohnsack ${ }^{1,4 \#}$, Katherine E. Bohnsack ${ }^{1 \#}$

${ }^{1}$ Department of Molecular Biology, University Medical Centre Göttingen, Humboldtallee 23, 37073 Göttingen, Germany

2 Department of Cellular and Molecular Medicine, University of Copenhagen, 3B Blegdamsvej, 2200N Copenhagen, Denmark

${ }^{3}$ Biosciences Institute, Newcastle University, Framlington Place, Newcastle upon Tyne, NE2 $4 \mathrm{HH}$, United Kingdom

${ }^{4}$ Göttingen Center for Molecular Biosciences, Georg-August University Göttingen, Justus-vonLiebig-Weg 11, 37077 Göttingen, Germany

\# Please address correspondence to:

Katherine E. Bohnsack

Address: Department of Molecular Biology, University Medical Centre Göttingen, Humboldtallee 23, 37073 Göttingen, Germany. Phone: +49-551-395978; Fax: +49-551395960. E-mail: katherine.bohnsack@med.uni-goettingen.de

Markus T. Bohnsack

Address: Department of Molecular Biology, University Medical Centre Göttingen, Humboldtallee 23, 37073 Göttingen, Germany. Phone: +49-551-395968; Fax: +49-551395960. E-mail: markus.bohnsack@med.uni-goettingen.de

\section{KEY WORDS}

RNA helicase, RNA modification, ribosome, ribosome biogenesis, small nucleolar RNA 


\section{ABSTRACT}

RNA helicases play important roles in diverse aspects of RNA metabolism through their functions in remodelling ribonucleoprotein complexes (RNPs), such as pre-ribosomes. Here, we show that the DEAD box helicase Dbp3 is required for processing of intron-encoded small nucleolar RNAs (snoRNAs) as well as efficient 2'-O-methylation of various sites within the $25 \mathrm{~S}$ ribosomal RNA (rRNA) sequence, and that numerous box C/D snoRNPs accumulate on preribosomes in its absence. Many snoRNAs guiding Dbp3-dependent rRNA modifications have overlapping pre-rRNA basepairing sites and therefore form mutually exclusive interactions with pre-ribosomes. Analysis of the distribution of these snoRNAs between pre-ribosomeassociated and "free" pools demonstrated that many are almost exclusively associated with pre-ribosomal complexes. Our data suggest that retention of such snoRNPs on pre-ribosomes when Dbp3 is lacking impedes rRNA 2'-O-methylation by reducing the recycling efficiency of snoRNPs and by inhibiting snoRNP access to proximal target sites. The observation of substoichiometric rRNA modification at adjacent sites suggests that the snoRNPs guiding such modifications interact stochastically rather than hierarchically with their pre-rRNA target sites. Together, our data provide new insights into the dynamics of snoRNPs on pre-ribosomal complexes and the remodelling events occurring during the early stages of ribosome assembly. 


\section{INTRODUCTION}

Production of eukaryotic ribosomes, which involves the assembly of four ribosomal RNAs (rRNAs) and 79 (Saccharomyces cerevisiae; yeast)/80 (human) ribosomal proteins, is one of the most energy-consuming cellular processes (1-3). In yeast, the best characterised model system for analysing ribosome assembly, this process requires the co-ordinated action of over 200 assembly factors that transiently associate with pre-ribosomal particles to facilitate correct assembly of the ribosomal subunits $(4,5)$.

A nascent pre-rRNA transcript (35S in yeast) containing three of the four rRNAs (18S, 5.8S and 25S) separated by internal transcribed spacers (ITS1 and ITS2) and flanked by external transcribed spacers (5' ETS and 3' ETS) is synthesised by RNA polymerase I (Pol I) in the nucleolus (6). The pre-rRNAs undergo various site-specific cleavages to release the mature rRNAs, several of which occur already co-transcriptionally, and an array of rRNA modifications are introduced $(7,8)$. The vast majority of rRNA modifications are 2'-O-methylations $(\mathrm{Nm})$ and pseudouridylations $(\Psi)$, which are largely introduced by small nucleolar RNPs (snoRNPs) composed of a small nucleolar RNA (snoRNA) and associated proteins $(9,10)$. SnoRNAs basepair with pre-rRNA sequences to direct the modification of specific target rRNA nucleotides by their associated methyltransferase (Nop1 (fibrillarin in humans)) or pseudouridine synthetase (Cbf5 (Dyskerin in humans)) $(9,11)$. Their extensive pre-rRNA interactions mean that snoRNPs likely also make important contributions to the early stages of ribosome assembly by regulating the order and dynamics of pre-rRNA folding, although how this takes place is currently poorly understood. Together with the various base methylations introduced by stand-alone modification enzymes, $\mathrm{Nm}$ and $\Psi$ cluster in functionally important regions of the ribosome, such as the peptidyl transferase centre, decoding site and intersubunit interface (12-14). Globally, rRNA modifications are suggested to contribute to the stability and conformational flexibility of ribosome structure and thereby influence translation efficiency and fidelity. The recent discovery of substoichiometric rRNA modifications in various species (8, 15-17) has revealed rRNA modifications as a source of ribosome heterogeneity and highlighted their potential roles in the translational control of gene expression (10).

During pre-rRNA maturation, various ribosomal proteins and biogenesis factors are recruited to the nascent pre-rRNA transcript giving rise to the small subunit (SSU) processome and early $90 S$ pre-ribosomal particles (18). Structural rearrangements and pre-rRNA cleavages separate the pre-40S (SSU) and pre-60S (large subunit, LSU) particles, which follow independent assembly pathways in the nucleolus and nucleoplasm (4). Maturation of both the pre-40S and pre-60S particles requires the dynamic association and dissociation of numerous assembly factors. Pre-40S particles containing the $20 \mathrm{~S}$ pre-rRNA are rapidly exported to the cytoplasm where final maturation and quality control steps take place. In contrast, nucleolar and 
nucleoplasmic maturation of pre-60S complexes involves numerous intermediate steps before the particles achieve export competence and can translocate to the cytoplasm $(19,20)$.

During their maturation, pre-ribosomal complexes undergo extensive remodelling, involving the establishment of rRNA folds present in mature ribosomes, a process which is often closely coupled with the recruitment and release of ribosomal proteins, assembly factors and snoRNPs. These structural rearrangements can serve as important checkpoints during the assembly pathway and energy-driven enzymes, such as AAA-ATPases, GTPases, kinases and ATP-dependent RNA helicases, have emerged as key regulators of these transitions (2123). RNA helicases are characterised by the presence of two RecA-like domains containing conserved sequence motifs involved in RNA binding, ATP binding and hydrolysis and the coupling of these to achieve unwinding (24). Of the $>20$ RNA helicases implicated in ribosome biogenesis in yeast (23), the majority function during the early stages of ribosome assembly, likely due to the more open conformation of pre-ribosomal particles at this stage, which allows greater access to their target sites for remodelling. While examples of helicases that are necessary for the association or dissociation of specific ribosomal proteins and assembly factors have been described (25-27), several RNA helicases have been implicated in modulating the dynamics of particular snoRNPs on pre-ribosomes. For example, Prp43 is required for the release of a specific subset of snoRNAs from early pre-LSU particles and is suggested to remodel such particles to facilitate the access of other snoRNPs $(28,29)$. Similarly, Dhr1, Has1, and Rok1 have all been linked to release of individual snoRNAs (U3, U14 and snR30 respectively) from pre-SSU complexes (28, 30-33). Nevertheless, the functions of other RNA helicases that act on pre-ribosomal complexes have remained largely unexplored.

Although Dbp3 has been implicated in pre-60S biogenesis, little is known about its role(s) in ribosomal subunit assembly. Here we show that the catalytic activity of the RNA helicase Dbp3 is required for efficient conversion of the $27 S_{A}$ pre-rRNA to $27 S_{B}$. This pre-rRNA processing defect is rescued by reduced expression of the box C/D snoRNP component Nop56, implying that the function of Dbp3 in pre-LSU maturation is linked to snoRNPs. Consistent with this, using northern blotting, RiboMeth-seq (RMS) and reverse transcription, quantitative PCR (RTqPCR) analysis of snoRNA levels on pre-ribosomes, we observe defects in snoRNA maturation, rRNA 2'-O-methylation and the pre-ribosomal accumulation of a subset of box $C / D$ snoRNPs in cells lacking Dbp3. We propose that Dbp3 is indirectly required for release of specific snoRNPs from pre-ribosomes, which is necessary for efficient snoRNP recycling and snoRNP access to proximal sites to enable stoichiometric rRNA modification. Our data implicate another RNA helicase, Prp43, in directly resolving the pre-rRNA basepairing of several snoRNAs guiding Dbp3-dependent rRNA 2'-O-methylations.

\section{MATERIALS AND METHODS}




\section{Molecular cloning}

The coding sequence of Dbp3, amplified from yeast genomic DNA (Supplementary Table S1), was cloned into a $\mathrm{PQE}-80$-based vector for the expression of proteins with an $\mathrm{N}$-terminal $\mathrm{His}_{10^{-}}$ $\mathrm{ZZ}$ tag in E. coli. To express a mutant version of Dbp3 from the same vector, site-directed mutagenesis using oligonucleotides listed in Supplementary Table S1 was performed to convert glutamate 263 to glutamine in the expressed protein (Dbp3 ${ }_{\mathrm{E} 263 \mathrm{Q}}$ ). For exogenous expression of Dbp3 in yeast, the coding sequence of Dbp3 and 500 basepairs upstream and downstream of it were cloned into pRS415 (Supplementary Tables S1 and S2). The Dbp3 $3_{\mathrm{E} 263 \mathrm{Q}}$ mutant was also created in this vector using site-directed mutagenesis. Overexpression of snR67 was achieved by cloning the sequence encoding the snoRNA and flanking regions into a dedicated snoRNA expression construct for expression of the snoRNA from within the intron of the actin gene under the control of a $\mathrm{PGAL}_{1}$ promoter (Supplementary Tables $\mathrm{S} 1$ and $\mathrm{S} 2$ ) (34).

\section{Expression of recombinant proteins in E. coli and purification}

Expression of His ${ }_{10}$-ZZ-Dbp3 or His ${ }_{10}-Z Z-D b p 33_{E 263 Q}$ was induced in BL21 codon plus cells by addition of $1 \mathrm{mM}$ Isopropyl $\beta$-D-1-thiogalactopyranoside (IPTG) for $16 \mathrm{~h}$ at $18^{\circ} \mathrm{C}$. Cells were pelleted and resuspended in Lysis buffer (50 mM Tris- $\mathrm{HCl}$ pH 7.0, $500 \mathrm{mM} \mathrm{NaCl}, 1 \mathrm{mM} \mathrm{MgCl}$, $10 \%$ glycerol, $10 \mathrm{mM}$ imidazole and $1 \mathrm{mM}$ phenylmethylsulfonyl fluoride (PMSF)). After disruption by sonication, the cell lysate was cleared by centrifugation at 20,000 x g for $30 \mathrm{~min}$ at $4^{\circ} \mathrm{C}$. Polyethylamine (PEI) was added to the soluble fraction to a concentration of $0.05 \%$ and the lysate was incubated at $4^{\circ} \mathrm{C}$ for $15 \mathrm{~min}$ before centrifuging at $33,000 \times \mathrm{g}$ for $30 \mathrm{~min}$ at $4^{\circ} \mathrm{C}$. The cleared lysate was incubated with cOmplete His-tag purification resin (Roche) for 2 $\mathrm{h}$ at $4^{\circ} \mathrm{C}$. After thorough washing steps with a buffer composed of $50 \mathrm{mM}$ Tris- $\mathrm{HCl} \mathrm{pH} \mathrm{7.0,500}$ $\mathrm{mM} \mathrm{NaCl}, 1 \mathrm{mM} \mathrm{MgCl} 2,10 \%$ glycerol and $30 \mathrm{mM}$ imidazole, bound proteins were eluted with a buffer containing $50 \mathrm{mM}$ Tris- $\mathrm{HCl} \mathrm{pH} 7.0,500 \mathrm{mM} \mathrm{NaCl}, 1 \mathrm{mM} \mathrm{MgCl}_{2}, 10 \%$ glycerol and 300 $\mathrm{mM}$ imidazole. The eluate was dialysed against a buffer containing $50 \mathrm{mM}$ Tris- $\mathrm{HCl} \mathrm{pH} 7.0$, $120 \mathrm{mM} \mathrm{NaCl}, 2 \mathrm{mM} \mathrm{MgCl}_{2}$ and $20 \%$ glycerol.

\section{In vitro ATPase assays}

$\mathrm{NADH}$-coupled assays were used to monitor the hydrolysis of $\operatorname{ATP}(35,36)$. Reactions containing $45 \mathrm{mM}$ Tris- $\mathrm{HCl} \mathrm{pH}$ 7.4, $25 \mathrm{mM} \mathrm{NaCl}, 2 \mathrm{mM} \mathrm{MgCl}, 1 \mathrm{mM}$ phosphoenolpyruvate, $300 \mu \mathrm{M}$ NADH, $20 \mathrm{U} / \mathrm{ml}$ pyruvate kinase and $4 \mathrm{mM}$ ATP were supplemented with $250 \mathrm{nM}$ recombinant His 10 -ZZ-tagged Dbp3/Dbp3 ${ }_{\mathrm{E} 263 \mathrm{Q}}$ and $0-1 \quad \mu \mathrm{M}$ RNA (5'GUAAUGAAAGUGAACGUAAAACAAAACAAAAC-3'). The absorbance at $340 \mathrm{~nm}$ was monitored using a BioTEK Synergy plate reader and the rate of ATP hydrolysis was calculated using the following equation where Kpath is the molar absorption co-efficient for a defined 
optical path length, which is defined as reaction volume (150 $\mu \mathrm{l} /$ well) and background NADH decomposition.

Yeast strains an ATPase rate $\left[\frac{\mathrm{ATP}}{\min }\right]=\frac{\frac{\mathrm{dA} 340}{\mathrm{dt}}\left[\frac{\mathrm{OD}}{\mathrm{min}}\right]}{\text { Kpath } * \text { moles ATP }}$

Yeast strains user

le S3 and were grown in YPD/G (1\% yeast extract, $2 \%$ peptone, $2 \%$ glucose/galactose) or synthetic media lacking leucine where appropriate. To generate the Dbp3 complementation system, the pRS415-based constructs for the expression of DBP3 from its endogenous promoter, or the empty pRS415 plasmid, were used to transform either wild type yeast or a $\triangle d b p 3$ strain. Yeast strains lacking individual snoRNA genes, or the SNR72-78 or SNR52-SNR67 clusters, were generated by substitution of the relevant gene with a marker cassette. Homologous recombination was similarly used to insert a truncated pGAL promoter ( $p G A L s$ ) and the sequence encoding an HA tag immediately upstream of the coding sequence of PRP43 to generate the PGALs-HAPrp43 strain. To deplete Prp43, cells of the pGALs-HA-Prp43 strain were grown in exponential phase in media containing galactose as the carbon source before switching to growth in media containing glucose for $8 \mathrm{~h}$.

\section{Protein extraction and western blotting}

Yeast cells were lysed by vortexing with glass beads and proteins were precipitated using 15\% trichloroacetic acid (TCA). Proteins were separated by SDS-PAGE and analysed by western blotting using the following primary antibodies (anti-Prp43 (kindly provided by Yves Henry); anti-HA (Sigma-Aldrich \# H3663); anti-Pgk1 (ThermoFisher Scientific \#459250)).

\section{RNA extraction and northern blotting}

Total RNA was extracted from exponentially growing yeast cells using acidic phenol and chloroform extracts as previously described (27). For analysis of long RNAs (>400 nt) by northern blotting, $6 \mu \mathrm{g}$ total RNA was separated by denaturing (glyoxal) agarose gel electrophoresis and transferred to a nylon membrane by vacuum blotting. Alternatively, to detect RNAs $<400 \mathrm{nt}$, total RNA was separated by denaturing ( $7 \mathrm{M}$ urea) polyacrylamide gel electrophoresis (PAGE) and transferred to a nylon membrane by electro-wet blotting. Membranes were then pre-hybridised in $0.25 \mathrm{M}$ sodium phosphate $\mathrm{pH} 7.0,7 \%$ SDS (w/v), 1 mM EDTA before addition of 5' [ ${ }^{32} \mathrm{P}$ ]-labelled DNA oligonucleotides (Supplementary Table S1) and incubation overnight at $37^{\circ} \mathrm{C}$. After washing steps, membranes were exposed to phosphorimager screens and signals detected using a Typhoon FLA9500 phosphorimager. 


\section{Sucrose density gradient centrifugation}

Sucrose density gradient centrifugation was essentially performed as previously described (37, 38). Whole cell extracts prepared from exponentially growing yeast cells by grinding in liquid nitrogen were clarified by centrifugation at $20,000 \times \mathrm{g}$ for $10 \mathrm{~min}$. Cleared lysates were separated on 10-45\% sucrose gradients in an SW-40Ti rotor for $16 \mathrm{~h}$ at $23,500 \mathrm{rpm}$. Fractions of $530 \mu \mathrm{l}$ were taken and RNA was extracted as described above for analysis by northern blotting or RT-qPCR.

\section{Analysis of snoRNA levels on pre-ribosomes by RT-qPCR}

Analysis of the relative proportions of 75 yeast snoRNAs between pre-ribosomal and nonribosomal complexes was performed as in (31). In brief, fractions derived from sucrose density gradient separation of (pre-) ribosomal and non-ribosomal complexes were pooled and RNA was extracted. RNAs were polyadenylated using E. coli poly $(A)$ polymerase (NEB) and then reverse transcribed using an oligo $d(T)$ primer and Superscript III reverse transcriptase (Thermo Fisher Scientific). RNA was digested by RNase $\mathrm{H}$ treatment and the cDNA was purified. Quantitative PCR was performed with oligonucleotides listed in (31) using the Mx3000P qPCR system (Agilent Technologies). The values obtained in samples from the $\triangle d b p 3$ strain were normalised to those from the wild type (WT) samples and ratios of preribosomal versus unbound levels for each snoRNA were calculated using the formula $2^{-(\mathrm{Ct}(\mathrm{WT})}$

- $\mathrm{Ct}(\Delta d b p 3)$ unbound)/2-(Ct(WT) - $\mathrm{Ct}(\Delta d b p 3)$ pre-ribosomal). Statistics for significance thresholds were determined as previously described (31).

\section{RiboMeth-seq}

RiboMeth-seq was essentially performed as previously reported (39). In brief, $5 \mu \mathrm{g}$ total RNA was fragmented under denaturing conditions using an alkaline buffer $(\mathrm{pH} 9.9)$. Subsequently, the RNA was separated on a denaturing (urea) polyacrylamide gel, fragments in the size of 20-40nt were excised and ligated to adapters using a modified tRNA ligase. cDNA was generated using Superscript III (Thermo Fisher Scientific) and sequenced on a PI Chip v3 using the lon Proton platform. Reads were mapped to the yeast rDNA and snoRNA sequences, and the RMS score (fraction methylated) was calculated as "score C" in Birkedal et al. (8). In a few cases, a barcode correction was applied when calculating the RMS score as described previously (40). The numbers of sequencing reads mapping to specific RNAs was used as a measure of their levels and is presented as reads per kilobase of transcript, per million mapped reads $(R P K M) \pm S E M$. Statistical significance was determined using Student's t-test $(p<0.05)$.

\section{RNase H-based cleavage assays for monitoring RNA 2'-O-methylation}


The methylation status of specific rRNA nucleotides was monitored using RNase $\mathrm{H}$-based cleavage assays (41). Chimeric 2'-O-methylated RNA-DNA oligonucleotides (Supplementary Table S4) were annealed to total RNA before treatment with RNase $\mathrm{H}$ (NEB) for 30 min at $37^{\circ} \mathrm{C}$. Reactions were stopped by addition of $240 \mathrm{mM} \mathrm{NaAc} \mathrm{pH} 5.2$ and $1 \mathrm{mM}$ EDTA before RNA extraction using phenol:chloroform:isoamylalcohol (25:24:1). Samples were separated by denaturing agarose gel electrophoresis and analysed by northern blotting.

\section{CLASH analysis of CRAC data}

Identification of snoRNA-rRNA hybrids in the CRAC data was performed using a bioinformatics pipeline developed for crosslinking, ligation and analysis of sequence hybrids (42). 


\section{RESULTS}

The catalytic activity of Dbp3 is required for production of the $\mathbf{2 7 S}_{\mathrm{B}}$ pre-rRNA.

Dbp3 has been shown to associate with early pre-LSU particles in vivo and to possess RNA duplex unwinding activity in vitro $(43,44)$, however the requirement for this catalytic activity for ribosome assembly has not been addressed. To establish a catalytically inactive mutant of Dbp3, wild type Dbp3 (Dbp3wt) and a mutant version in which glutamate 263 within the conserved DEAD motif responsible for ATP binding and hydrolysis was substituted for glutamine $\left(\mathrm{Dbp}_{\mathrm{E} 263 \mathrm{Q}}\right)$ were recombinantly expressed with $\mathrm{N}$-terminal $\mathrm{His}_{10} \mathrm{ZZ}$ tags in Escherichia coli (E. coli) and purified by nickel affinity chromatography (Figure $1 \mathrm{~A}$ and B). In vitro NADH-coupled ATPase assays were then used to monitor the rates of ATP hydrolysis by these two proteins in the presence of different amounts of RNA. Compared to a control sample without protein, Dbp3wT, but not Dbp3 ${ }_{\mathrm{E} 263 \mathrm{Q}}$, showed minimal ATPase activity in the absence of RNA (Figure 1C). Addition of increasing concentrations of RNA stimulated the rate of ATP hydrolysis by Dbp3wT, but Dbp3 $3_{\mathrm{E} 263 \mathrm{Q}}$ never hydrolysed ATP above the background level (Figure 1C), confirming that this amino acid substitution abolishes ATP hydrolysis by the protein. Next, to explore the requirement for this catalytic activity for ribosome biogenesis, a yeast complementation system was generated. Wild type BY4741 yeast or a strain where $D B P 3$, which is not essential, had been deleted from the genome $(\triangle d b p 3)$ were transformed with pRS415-based constructs for the expression of untagged Dbp3wT or Dbp3 ${ }_{\mathrm{E} 263 \mathrm{Q}}$ from the endogenous $D B P 3$ promoter, or as a control, the empty plasmid. Pre-rRNAs from these strains were analysed by northern blotting using a mixture of probes hybridising within the ITS1 or ITS2 regions of the pre-rRNA transcript (Figure 2A). Compared to the wild type strain carrying an empty pRS415 vector, cells lacking Dbp3 showed elevated levels of the 35S, 33S/32S and $27 S_{A}$ pre-rRNAs as well as a markedly reduced level of the $27 S_{B}$ pre-rRNA (Figure $2 B$ ). The significantly increased $27 S_{A}-27 S_{B}$ ratio in the $\Delta d b p 3$ strain indicates that $D b p 3$ is required for the efficient conversion of $27 S_{A}$ to $27 S_{B}$, which occurs by processing at the $A_{3}, B_{1}$ and $B_{2}$ sites. This result is consistent with a previous analysis indicating the requirement for Dbp3 for processing at the $A_{3}$ site (43). Importantly, these pre-rRNA processing defects were rescued by expression of plasmid-derived Dbp3 ${ }_{\mathrm{wT}}$, confirming that they are caused by the lack of Dbp3. In contrast, expression of exogenous Dbp3 ${ }_{\mathrm{E} 263 \mathrm{Q}}$ in the $\Delta d b p 3$ background showed increased levels of the $35 \mathrm{~S}$ and $27 \mathrm{~S}_{A}$ pre-rRNAs and loss of the $27 \mathrm{~S}_{B}$ intermediate (Figure $2 \mathrm{~B}$ ), demonstrating that the catalytic activity of $\mathrm{Dbp3}$ is necessary for its function in LSU biogenesis.

\section{The role of Dbp3 in LSU biogenesis is linked to box C/D snoRNPs.}

Interestingly, Dbp3 was identified in a high-throughput screen to identify regulators of rRNA 2'$O$-methylation (Nicholas Watkins and Robert van Nues, unpublished). This suggested that the pre-rRNA processing defects observed in the absence of Dbp3 and its function in ribosome 
assembly may be linked to rRNA 2'-O-methylation-guiding box C/D snoRNPs. To test this hypothesis, yeast strains were generated in the wild type and $\Delta d b p 3$ backgrounds where expression of the essential core box C/D snoRNP component Nop56 is reduced by introduction of an antibiotic resistance cassette into the 3' untranslated region (UTR) of the NOP56 gene, leading to destabilisation of the transcribed mRNA (NOP56 DAmP) (45). Analysis of pre-rRNA processing confirmed the pre-rRNA processing defects previously observed in cells lacking Dbp3 (Figure 2B and C) and revealed that a reduced level of Nop56 leads to defects in early pre-rRNA processing, characterised by accumulation of the $35 \mathrm{~S}$ and $23 \mathrm{~S}$ pre-rRNAs as well as reduced levels of the $20 \mathrm{~S}$ and $27 \mathrm{~S}_{\mathrm{A} / \mathrm{B}}$ intermediates (Figure $2 \mathrm{C}$ ). In the $\triangle d b p 3+N O P 56_{\mathrm{DAmP}}$ strain, the 35S pre-rRNA and aberrant $23 S$ species were observed to accumulate and the amount of the $20 \mathrm{~S}$ processing intermediate was reduced, consistent with the lack of Nop56 in this strain. However, the amount of $27 \mathrm{~S}_{\mathrm{A}}$ and $27 \mathrm{~S}_{\mathrm{B}}$, and the ratio between these processing intermediates was comparable to that in wildtype cells (Figure $2 \mathrm{C}$ ). The finding that codepletion of Nop56 rescues the pre-rRNA processing phenotype caused by lack of Dbp3 supports the notion that Dbp3 is functionally linked to rRNA methylation.

\section{Dbp3 is required for 2'-O-methylation of specific nucleotides within the 25S rRNA sequence.}

To explore the link between Dbp3 and rRNA 2'-O-methylation in more detail, RiboMeth-Seq (RMS) analysis (8) was performed on RNAs from wild type cells and those lacking Dbp3. RNAs were subjected to partial alkaline hydrolysis, which does not cleave 2'-O-methylated nucleotides, and the resultant RNA fragments were copied into a cDNA library that was subjected to lon Torrent sequencing (39). After mapping of the obtained sequencing reads to the $S$. cerevisiae transcriptome, the number of read ends mapping to each nucleotide of the $18 S$ and 25S rRNA sequences was determined and used to calculate an RMS score for each 2'-O-methylated nucleotide. Consistent with previous analyses (8), in wild type yeast, the majority of 2'-O-methylated sites were almost fully modified, with only few exceptions (Figure $3 A$ and B). While the extent of modification at only one site, Um1269, within the 18S rRNA of the SSU was affected by lack of Dbp3 (Figure 3A), strikingly, lack of Dbp3 caused clear reductions in the extent of 2'-O-methylation at various (19/37) sites within the 25S rRNA (Figure 3B). Dbp3-dependent 2'-O-methylations are present along the length of the 25S rRNA sequence and are interspersed with non-Dbp3-dependent modifications. As no structural information on pre-ribosomal complexes bound by Dbp3 is currently available, the affected 2'O-methylations were mapped on the tertiary structure of the 25S rRNA in the earliest pre-LSU complex where the complete rRNA sequence is visible, which is a pre-60S particle purified via the nucleolar/nucleoplasmic GTPase Nog2 (46). This showed that while these modifications 
are all close to the peptidyltransferase centre and tRNA binding sites of the ribosome, they do not strictly cluster in one specific region (Figure $3 C$ ).

We next addressed the question of whether the catalytic activity of Dbp3, or merely its presence, is required for rRNA 2'-O-methylation. While RNase $\mathrm{H}$ efficiently cleaves RNA in RNA-DNA hybrids, its cleavage activity is inhibited by the presence of RNA 2'-O-methylation. The extent of modification at specific sites can therefore be monitored using chimeric RNADNA oligonucleotides targeting different modified nucleotides in the rRNA and RNase $\mathrm{H}(41)$. Cleavage assays monitoring three Dbp3-dependent 2'-O-methylation sites (25S-Am876, 25SUm898 and 25S-Um2724) were first performed on RNAs derived from wild type yeast and cells lacking the snR72 (snR72-78 cluster), snR40 or snR67 snoRNAs that guide these modifications to verify the effectiveness of the method. Northern blotting analysis of the reaction products using probes hybridising upstream and/or downstream of the cleavage sites revealed no specific cleavage of the 25S rRNA in the absence of RNase $\mathrm{H}$ or in RNA derived from wild type yeast, consistent with the RMS data showing that these sites are normally almost fully modified. However, reduced levels of the full length 25S rRNA and the generation of products of sizes corresponding to the expected cleavage fragments was observed for RNAs lacking the snoRNA guiding each modification under investigation (Supplementary Figure S1). Cleavage assays were then performed on RNAs purified from wild type yeast or $\Delta d b p 3$ cells complemented with an empty vector $(E V)$ or plasmids for the expression of wild type Dbp3 (Dbp3wt) or catalytically inactive Dbp3 (Dbp3 $\left.{ }_{\mathrm{E} 263 \mathrm{Q}}\right)$. Again, no specific cleavage activity was observed in the absence of RNase $\mathrm{H}$ and in the presence of RNase $\mathrm{H}$, the $25 \mathrm{~S}$ rRNA remained uncleaved in the wild type strain carrying an empty vector (Figure 3D-F). In contrast, the 25S rRNA derived from $\Delta d b p 3$ cells complemented with either the empty vector or the plasmid for expression of $\mathrm{Dbp} 3_{\mathrm{E} 263 \mathrm{Q}}$ was partially cleaved by RNase $\mathrm{H}$ leading to reduced levels of the full length 25S rRNA and generation of the expected cleavage fragments (Figure 3D-F). rRNA 2'-O-methylation of these sites was largely restored by expression of plasmid-derived $\mathrm{Dbp} 3 \mathrm{wt}_{\mathrm{w}}$ in the $\Delta d b p 3$ strain as only minimal cleavage was observed. The reduced 2'-O-methylation of 25S-Am876, 25S-Um898 and 25S-Um2724 upon expression of $\mathrm{Dbp3}_{\mathrm{E} 263 \mathrm{Q}}$ supports a role for the catalytic activity of Dbp3 in rRNA 2'-O-methylation.

\section{Dbp3 is required for maturation of the intron-derived U18 and U24 snoRNAs.}

The substoichiometric 2'-O-methylation of numerous nucleotides within the 25S rRNA in the absence of Dbp3 raised the possibility that the cellular levels of the snoRNAs that guide these modifications are reduced when Dbp3 is lacking. The numbers of sequencing reads from the RMS analysis of wild type and $\Delta d b p 3$ cells mapping to each yeast snoRNA were used to compare snoRNA levels in these two strains. Lack of Dbp3 was not observed to cause any significant differences in snoRNA levels and northern blotting analysis of the subset of 
snoRNAs guiding Dbp3-dependent rRNA 2'-O-methylations confirmed this finding (Supplementary Figure S2A and B). This demonstrates that altered snoRNA levels are not the basis of the reduced rRNA 2'-O-methylation detected in $\triangle d b p 3$. However, while the overall levels of the U18 and U24 snoRNAs were not affected by lack of Dbp3, northern blotting for the U18 snoRNA revealed the presence of two species and deletion of DBP3 caused a shift towards the longer form (Supplementary Figure S2B and Figure 4A). Similarly, it appeared that a longer form of U24 accumulated upon deletion of DBP3. In contrast to the majority (73/79) of yeast snoRNAs that are synthesised as mono- or poly-cistronic transcripts, U18 and U24 are encoded within the introns of protein coding pre-mRNAs from which they are released by debranching and/or endonucleolytic cleavage, followed by exonucleolytic processing $(47,48)$. To investigate the nature of the longer U18 and U24 versions, northern blotting was performed on total RNA extracted from wild type yeast and cells lacking either Dbp3 or the 3'-5' exonuclease Rrp6, which is implicated in 3' end processing of snoRNAs (49). Only the longer version of $\mathrm{U} 18$, enriched in $\Delta d b p 3$, was present in the $\Delta r r p 6$ strain, suggesting that Dbp3 is required for efficient 3 ' maturation of the U18 snoRNA (Figure 4A). To verify this, the RMS sequencing reads from wild type and $\Delta d b p 3$ datasets were mapped to the annotated U18 sequence $+/-50 \mathrm{nt}$ and the number of reads mapping to each nucleotide was determined. Interestingly, for U18 from wild type cells, this revealed high sequence coverage from the 5' $\mathrm{G}$, which forms the first basepair of the terminal stem, to $8 \mathrm{nt}$ beyond the annotated 3' end of the snoRNA. Sequence coverage was also observed for a further $13 \mathrm{nt}$ (Figure 4B and C). Strikingly, the numbers of sequencing reads mapping to nucleotides beyond the annotated 3' end of the U18 snoRNA were notably higher in the $\Delta d b p 3$ strain than in wild type yeast (Figure $4 \mathrm{~B}$ and $\mathrm{C}$ ), confirming the requirement for Dbp3 for 3 ' end processing of this snoRNA. Northern blotting analysis revealed that extended form of U24 accumulated when Dbp3 is lacking was of an intermediate length between that detected in the wild type and $\Delta r r p 6$ strains (Figure 4A). This suggests that a 3' extended U24 precursor is only partially processed in the absence of Dbp3, however, this could not be confirmed by analysis of RMS sequencing reads (Supplementary Figure $3 \mathrm{~A}$ and $\mathrm{B}$ ). In contrast to pre-U18, where the $5^{\prime}$ and 3 ' ends form a relatively weak terminal stem structure, pre-U24 forms a strong 7-8 basepair terminal stem (Figure 4D and Supplementary Figure 3C). As alkaline hydrolysis, which is used to generate RNA fragments in the RMS procedure, is impaired by nucleotide basepairing, it is possible that the presence of the terminal stem structure of pre-U24 reduces read coverage of the precursor sequences, preventing detection of the effect of $\mathrm{Dbp} 3$ on 3' end processing. Interestingly, both $\mathrm{U} 18$ and U24 could be processed to their mature forms in cells expressing Dbp3 ${ }_{\mathrm{E} 263 \mathrm{Q}}$ (Figure $4 \mathrm{E})$, indicating that the catalytic activity of Dbp3 is not required for its function in U18 and U24 snoRNA biogenesis. 
Given these findings, it is possible that the reduced 2'-O-methylation of the rRNA nucleotides guided by these snoRNAs (U18 - 25S-Am649, 25S-Am650, U24 - 25S-Cm1437, 25S-Am1449, 25S-Gm1450) arises due to the defects in snoRNA biogenesis. However, although lack of Dbp3 strongly impairs production of the mature U18 and U24 snoRNAs (Figure 4A), the RMS data implies that partial 2'-O-methylation of the U18/U24-guided sites can still occur, and that the extent of modification at the $2 / 3$ sites guided by each snoRNA differ (Figure 3B). Therefore, to investigate whether the 3' extended forms of U18 and U24 may be functional, their interactions with the 2'-O-methyltransferase Nop1 and their associations with pre-ribosomes were examined. Nop1-containing complexes were isolated from cell extracts prepared from wild type yeast expressing His-TEV protease cleavage site-Protein A (HTP)-tagged Nop1 or the equivalent strain lacking Dbp3. This revealed a similar enrichment of both processed and unprocessed U18 and U24 with Nop1 from the two strains, implying that both snoRNA versions are incorporated into snoRNPs (Figure 4F). The distribution of (pre-)U18 and (pre-)U24 between pre-ribosome-associated and non-pre-ribosome-associated pools was then examined. Whole cell extracts prepared from wild type yeast or cells lacking Dbp3 were subjected to sucrose density centrifugation and RNAs present in each fraction were examined by northern blotting (Figure 4G). This revealed that both the mature and precursor forms of U18 and U24 associate with pre-ribosomes. It is not possible to exclude that although the immature versions of the U18 and U24 snoRNPs associate with pre-ribosomes, they are not functional. However, it has previously been shown that the U24 snoRNA within its intron lariat is capable of directing rRNA modification (50), suggesting that the short 3' snoRNA extension observed when Dbp3 is lacking probably does not influence the ability of these snoRNPs to mediate rRNA 2'-O-methylation. It is likely, therefore, that there is an alternative mechanistic basis for the rRNA modification defects observed at the U18 and U24-guided sites when Dbp3 is absent.

\section{A subset of box $C / D$ snoRNAs accumulate on pre-ribosomes when Dbp3 is lacking.}

As snoRNA levels and the processing of most snoRNAs are not affected by lack of Dbp3, an explanation for the reduced rRNA 2'-O-methylations observed in $\triangle d b p 3$ could be that access of these snoRNAs to pre-ribosomes is impaired when $D B P 3$ is deleted. Therefore, to quantitatively monitor snoRNA levels on pre-ribosomes, whole cell extracts prepared from wild type yeast or cells lacking Dbp3 were subjected to sucrose density centrifugation. Fractions containing (pre-)ribosomal complexes and those containing non-ribosome associated proteins, RNAs and small complexes were identified based on the absorbance at $260 \mathrm{~nm}$ and were pooled. RNA extracted from these two pools was reverse transcribed to produce cDNA, which served as a template for qPCR to determine the levels of each of the 75 snoRNAs present in yeast $(27,31)$. The ratio of pre-ribosome-associated to non-ribosome-associated RNA was 
determined for each snoRNA and compared between the wild type and $\Delta d b p 3$ strains. A value of 1 reflects no variation between the relative proportions of non-ribosome-associated and preribosomal snoRNA in the two strains while higher values reflect snoRNA accumulation on preribosomes when Dbp3 is lacking and lower values indicate reduced amounts of pre-ribosomal snoRNA in $\Delta d b p 3$. The values 2 and 0.5 were calculated as upper and lower statistical significance thresholds for accumulation on and exclusion from pre-ribosomes respectively and the values obtained for most snoRNAs varied within these limits. However, several box C/D snoRNAs (U18, U24, snR39, snR39b, snR50, snR55, snR59, snR60, snR61, snR67, snR69, snR74 and snR79) significantly accumulated on pre-ribosomes in the absence of Dbp3, whereas no specific changes in the levels of any of the H/ACA box snoRNAs were detected (Figure 5 and Supplementary Figure S4). Intriguingly, 10 of the 13 snoRNAs (U18, U24, snR39, snR39b, snR50, snR55 snR59, snR60, snR67 and snR69) that accumulate on pre-ribosomes when Dbp3 is lacking guide Dbp3-dependent 2'-O-methylations, implying that alterations in snoRNA levels on pre-ribosomes may indeed underlie the defects in rRNA 2'-Omethylation observed in $\Delta d b p 3$. These results do not, however, support the initial hypothesis that lack of Dbp3 affects rRNA 2'-O-methylation by directly impairing access of the snoRNAs guiding these modifications to pre-ribosomes as the amount of these snoRNPs on preribosomes was increased rather than reduced.

\section{Alterations in snoRNA dynamics on pre-ribosomes when Dbp3 is lacking affect rRNA 2'-O-methylation at specific sites.}

The extensive interactions snoRNAs form with their rRNA substrates involve both the snoRNA guide sequence as well as extra basepairing regions that stabilise snoRNA-pre-rRNA interactions to promote rRNA modification $(11,51)$. Mapping of the snoRNA-rRNA basepairing of the Dbp3-dependent snoRNAs onto the secondary structure of the 25S rRNA sequence (52) revealed that many of these snoRNAs have partially overlapping basepairing sites (Figure 6A$C$ and Supplementary Figure S5). Pre-rRNA basepairing by these snoRNAs will therefore be mutually exclusive and failure to release one snoRNP after modification would be expected to impede access of the snoRNP with an overlapping basepairing site, leading to reduced 2'-Omethylation of the adjacent site. Assuming stochastic recruitment of such snoRNAs to preribosomes, this would result in both snoRNA accumulation on pre-ribosomes and substoichoimetric rRNA 2'-O-methlyation. Notably, the U18 snoRNP is proposed to exists in two alternative conformations to target the adjacent sites (34) (Figure 6C) and if so, failure to release the snoRNP from one target site would impede modification of the other site by preventing access of the snoRNP in its alternative conformation. It is possible, therefore, that the model of snoRNA retention impairing modification at proximal sites is not only applicable 
for modifications installed by different snoRNPs, but that competition could also occur between differently assembled forms of a single snoRNP.

Retention of a snoRNP impairing modification at a proximal site can explain many of the effects on pre-ribosomal snoRNA levels and rRNA 2'-O-methylation observed by RT-qPCR and RMS, but several of the Dbp3-dependent modifications are introduced by snoRNPs that do not have overlapping basepairing sites on the pre-rRNA and/or do not accumulate on pre-ribosomes in the absence of Dbp3. Therefore, other mechanisms likely also contribute to regulation of rRNA modification by Dbp3. For example, the pre-rRNA basepairing sites of snR76 and snR67 do not overlap with those of any other snoRNA affected by $\Delta d b p 3$ (Figure 6D and E), and the snR40, snR72, snR73, snR76 and snR78 snoRNPs guide Dbp3-dependent rRNA 2'-Omethylations but do not accumulate on pre-ribosomes in the absence of Dbp3 (Figure 3B and Figure 5). Pre-rRNA modification by snoRNPs is a dynamic process in which snoRNPs are transiently recruited to pre-ribosomes but after modification, they are released and recycled to other pre-ribosomal particles to install further modifications. It is therefore possible that retention of snoRNPs on particular pre-ribosomal particles when Dbp3 is lacking reduces the amount of "free" snoRNP available for (re-)recruitment to other pre-ribosomes thus leading to impaired rRNA 2'-O-methylation. To explore this possibility further, we examined the proportions of snoRNAs guiding Dbp3-dependent rRNA modifications that are pre-ribosomeassociated in wild type cells using sucrose density gradient separation followed by northern blotting. This revealed that some of these snoRNAs (e.g. U18, snR39, snR50, snR59 and snR69) are present in both pre-ribosome-associated and non-ribosome-associated pools, while others (e.g. U24, snR40, snR60, snR67, snR72, snR73 and snR76) are almost exclusively present on pre-ribosomes (Figure 6F). On the one hand, this result may explain why not all snoRNAs guiding Dbp3-dependent modifications are observed to accumulate on pre-ribosomes in $\Delta d b p 3$, as the absence of a free pool of snoRNA even in wild type cells will prevent significant increases in the amount of pre-ribosomal snoRNA when Dbp3 is lacking. On the other hand, these data support the model that inefficient recycling of snoRNAs that are largely pre-ribosome-associated could reduce the amount of available snoRNP below the critical threshold required for stoichiometric rRNA modification of nascent particles. In the case of snoRNPs such as U24, snR50, snR72, snR40 and snR60 that have both overlapping basepairing sites on the pre-rRNA and are almost exclusively pre-ribosome-associated, it is likely that both impaired snoRNP access caused by retention of a snoRNP targeting a proximal site as well as limiting amounts of available snoRNP due to compromised recycling contribute to the reduced rRNA 2'-O-methylation observed in $\triangle d b p 3$.

\section{Overexpression of a snoRNA rescues the methylation defect caused by lack of Dbp3.}

To explore how changes in the available amount of a snoRNA that accumulates on preribosomes in the absence of Dbp3 influence rRNA 2'-O-methylation, we focused on snR67. 
This snoRNP installs two modifications within the 25S rRNA sequence that are distant on the linear rRNA sequence (snR67 - 25S-Gm2619 and 25S-Um2724). The basepairing snR67 forms to guide $25 S-G m 2619$ is spatially distinct from the basepairing regions of any other snoRNA and the basepairing snR67 forms to introduce the 25S-Um2724 modification only weakly overlaps with that of snR51, a snoRNA unaffected by Dbp3. Notably, snR67 is largely pre-ribosome-associated in wild type cells and is almost exclusively associated with these complexes in the absence of Dbp3 (Figure 6F and Supplementary Figure S6A). This suggests that alterations in the dynamics of the available snR67 between its two modification sites would likely affect the extent of 2'-O-methylation of these positions. A plasmid for the overexpression of snR67 from within the intron of the actin pre-mRNA, under the control of a $p G A L_{1}$ promoter was generated and used to produce wild type and $\Delta d b p 3$ yeast strains capable of overexpressing snR67 (Supplementary Figure S6B) (34). RNase H-based cleavage assays were then performed to monitor the extent of 2'-O-methylation of 25S-Gm2619 and 25SUm2724. The 25S rRNA remained intact in the absence of RNase $\mathrm{H}$ or when assays were performed on total RNA derived from wild type cells carrying an empty plasmid or overexpressing snR67 (Figure 7A and B). As previously, the 25S rRNA derived from cells lacking Dbp3 with normal snR67 levels was cleaved by RNase $\mathrm{H}$ indicating reduced 2'-Omethylation of 25S-Gm2619 and 25S-Um2724 (Figure 7A and B). Upon overexpression of snR67, however, the rRNA modification defects caused by lack of Dbp3 were rescued (Figure $7 A$ and $B$ ), suggesting that failure to release snR67 from one of its pre-rRNA basepairing sites in the absence of Dbp3 limits the amount of snoRNP available to basepair with and modify its other target site.

\section{The DEAH box RNA helicase Prp43 likely directly unwinds the pre-rRNA basepairing of a subset of snoRNAs guiding Dbp3-dependent rRNA 2'-O-methylations.}

Intriguingly, several of the snoRNAs that are retained on pre-ribosomes when Dbp3 is absent have previously been shown to accumulate on pre-ribosomes when the RNA helicase Prp43 is lacking (snR59, snr50, snR39, snR39b and U18) (28), suggesting that these helicases both contribute to facilitating release of a subset of snoRNAs from pre-ribosomes. We therefore investigated whether, similar to Dbp3, lack of Prp43 causes reduced 2'-O-methylation of sites proximal to those guided by the retained snoRNAs. A yeast strain was constructed in which expression of Prp43 is under the control of the galactose-inducible/glucose-repressible pGALs promoter. Importantly, in the presence of galactose, Prp43 is expressed close to its endogenous level and growth in glucose-containing media leads to efficient depletion of the protein after 6-8 h (Figure 8A and B). RMS analysis of cells grown in the presence of galactose and glucose revealed extensive effects on rRNA 2'-O-methylation with the RMS scores for 7 of the 18 sites in the $18 \mathrm{~S}$ rRNA and 24 of the 37 sites in the $25 \mathrm{~S}$ rRNA reduced $>10 \%$ upon 
depletion of Prp43 (Figure 8C and D). Analysis of snoRNA levels in these datasets showed that $19 / 79$ and $3 / 79$ snoRNAs were significantly up- and down-regulated in the pGALs-HAPrp43 strain grown in the galactose- and glucose-containing media respectively (Supplementary Figure 7). However, with the exception of U24, these snoRNAs do not guide Dbp3-dependent rRNA 2'-O-methylations, and it is likely that the observed changes in snoRNA expression levels arise due to the media exchange rather than depletion of Prp43. Notably, 11 2'-O-methylations within the 25S rRNA affected by depletion of Prp43 and guided by snoRNAs whose expression level was not significantly altered, were also reduced in the absence of Dbp3, further supporting the model linking snoRNA retention to impaired rRNA methylation.

The finding that lack of either Dbp3 or Prp43 individually leads to snoRNA retention on preribosomes and impaired rRNA methylation implies that these proteins do not act redundantly, but rather suggests that they regulate snoRNA dynamics on pre-ribosomes in different ways. Transcriptome-wide identification of the RNA binding sites of Prp43 showed that this helicase crosslinks to many of the snoRNAs that accumulate on pre-ribosomes when the Prp43 or Dbp3 are lacking, as well as to their pre-rRNA target sites (28), suggesting that Prp43 may play a direct role in releasing these snoRNAs by resolving their basepairing with the pre-rRNA. This model necessitates that Prp43 simultaneously contacts both the snoRNA and pre-rRNA sequences involved in the basepairing interaction. To determine if this is the case, further bioinformatic analysis of the Prp43 CRAC dataset using the Hyb bioinformatic pipeline (42) was performed to identify chimeric sequencing reads generated by the ligation of physically proximal sequences during the CRAC procedure. This revealed numerous such chimeric reads containing the sequences of Dbp3/Prp43-dependent snoRNAs and their pre-rRNA target sites within the Prp43 CRAC dataset (Figure 8E). These findings strongly suggest that Prp43 binds to these RNA sequences when they are basepaired, thereby supporting a direct role for the helicase in unwinding of these snoRNA-pre-rRNA duplexes. 


\section{DISCUSSION}

Dbp3 is an exception among the RNA helicases involved in ribosome assembly as it is not an essential protein (43), but here, we demonstrate the requirement of its catalytic activity for efficient processing of the $27 S_{A}$ pre-rRNA and uncover a role in regulating rRNA modification. Our data show that approximately half of the 2'-O-methylations in the 25S rRNA are introduced substoichiometrically in the absence of Dbp3. Furthermore, a subset of box C/D snoRNAs, many of which guide Dbp3-dependent rRNA 2'-O-methylations, accumulate on pre-ribosomes when the helicase is lacking. Close inspection of the interactions snoRNAs guiding Dbp3dependent modifications make with the pre-rRNA and analysis of the distribution of these snoRNAs between pre-ribosome-associated and non-ribosomal pools revealed that these snoRNAs either have overlapping basepairing sites on the pre-rRNA and/or are predominantly pre-ribosome-associated. We therefore propose that Dbp3 facilitates the release of a subset of snoRNAs from pre-ribosomes and that retention of these snoRNAs in the absence of Dbp3 impedes the access of other snoRNPs to proximal sites and impairs snoRNP recycling such that snoRNAs become limiting for rRNA modification. Together these two mechanisms lead to reduced 2'-O-methylation of specific rRNA sites.

A role for Dbp3 in the biogenesis of the intron-encoded snoRNAs U18 and U24 was also uncovered. However, the findings that pre-U18 and pre-U24 associated with Nop1 and preribosomes, together with partial modification of the U18 and U24 target sites in the absence of Dbp3, implies that this additional function of Dbp3 is not the sole basis of the methylation defects observed at these positions. This is further supported by the finding that the catalytic activity of Dbp3 appears to be required for rRNA 2'-O-methylation, while expression of catalytically inactive Dbp3 was sufficient to restore processing of U18 and U24. 3' extended forms of U18 and U24 are observed when a KK(E/D) motif of GNO1 is deleted (53) and intriguingly, this motif is also present in Dbp3 as well as the box C/D snoRNP proteins Nop56 and Nop58. This suggests that an interplay between factors containing this motif, possibly together with an unknown protein binding the $\mathrm{KK}(\mathrm{E} / \mathrm{D})$ motif, may underlie the observed processing defects.

Interestingly, many of the effects of $D B P 3$ deletion on snoRNA levels on pre-ribosomes, especially those that guide 2'-O-methylations within domain I, are also observed when the RNA helicase Prp43 is depleted. Similarly, although depletion of Prp43 has a broader effect on rRNA 2'-O-methylation than lack of Dbp3, perhaps due to its functions in pre-mRNA splicing as well as ribosomes biogenesis, reduced levels of either of these helicases impacts a largely overlapping set of modifications. It is possible that these common phenotypes occur due to the action of Prp43 and Dbp3 on pre-LSU complexes at the same stage of maturation and reflect general effects caused by impairing the assembly pathway at this point. However, these effects 
on pre-ribosomal snoRNA levels are not observed upon depletion of other LSU biogenesis factors whose depletion causes pre-rRNA processing defects similar to lack of Dbp3 or Prp43, e.g. Mak5 (27). The data therefore rather suggest that Dbp3 and Prp43 both contribute to regulating the dynamics of a subset of snoRNAs on pre-ribosomes, thereby influencing rRNA 2'-O-methylation. Dbp3 and Prp43 belong to the DEAD and DEAH box families of RNA helicases respectively. Mechanistically, DEAH box helicases, including Prp43, act processively and are able to translocate along RNA strands (54) making them highly suitable enzymes for directly unwinding multiple snoRNA-pre-RNA duplexes. Indeed, mining available Prp43 CRAC datasets (28) indicated the crosslinking of Prp43 to snoRNAs when they are basepaired to their pre-rRNA target sites, supporting a direct role for Prp43 in resolving these duplexes. In contrast to DEAH box helicases, DEAD box proteins generally remodel their substrates by inducing local strand unwinding (55). While several DEAD box helicases are implicated in snoRNA release from pre-ribosomes, in these cases, each helicase is only linked to an individual snoRNA e.g. Has1 and U14, and Rok1 and snR30 [26,29,32]. Consistent with action by local strand unwinding, Dbp3 has been shown to be capable of unwinding an RNA duplex of 10 nucleotides (nt) in vitro (44). Although this duplex is similar in length to the basepairing interactions box C/D snoRNAs form with their pre-rRNA targets (10-19 nt, in the case of Dbp3-dependent snoRNAs), a direct role of Dbp3 in releasing the 13 snoRNAs that accumulate on pre-ribosomes in its absence would necessitate numerous and extensive conformational changes of Dbp3 on the pre-ribosomes to enable its catalytic site to directly contact each of the snoRNA-pre-rRNA duplexes. Even within the highly dynamic process of ribosome assembly, this extent of re-positioning or dissociation/re-association of a single protein is improbable, implying that, in contrast to Prp43, Dbp3 likely affects snoRNA dynamics indirectly. We anticipate that rearrangement of a particular pre-rRNA region by Dbp3 induces long-range effects on pre-ribosome structure that impede the release of specific snoRNPs that basepair with spatially distinct pre-rRNA sequences. It is important to note that the early preLSU particles bound by Dbp3 are highly dynamic structures and therefore lack of this helicase could influence the dynamics of any of the numerous pre-ribosome maturation events that are occurring simultaneously on such particles. Accordingly, it is likely that the impaired processing of the $27 \mathrm{~S}_{\mathrm{A}}$ pre-rRNA observed upon deletion of $D B P 3$ is a downstream consequence of failure of release of specific snoRNPs during preceding pre-LSU maturation steps. Despite extensive efforts to identify the pre-rRNA binding site of Dbp3 using the UV crosslinking and analysis of cDNA (CRAC (57)) approach and its derivatives, this has so far not been possible. On the one hand, this may be due to the insensitivity of the interacting Dbp3 amino acids and pre-RNA sequences to crosslinking but, on the other hand, it may reflect a very transient interaction between Dbp3 and the pre-ribosome. Structural analysis of pre-ribosomal particles containing Dbp3 that allow visualisation of the helicase active site on its target RNA sequence(s) will 
therefore likely be required to gain further mechanistic insight into the precise pre-ribosome remodelling event(s) regulated by Dbp3.

Analyses of the timing of rRNA 2'-O-methylation demonstrate that the majority of these modifications are introduced co-transcriptionally $(7,8)$. As these modifications cluster within the rRNA sequences that form functionally important regions of the pre-ribosome and box $C / D$ snoRNAs form extensive basepairing with their pre-rRNA substrates, it is intuitive that snoRNPs mediating proximal modifications must form mutually exclusive interactions with preribosomal particles. However, it has remained unclear whether such "overlapping" snoRNPs act hierarchically or stochastically. Our detection of correlated mild accumulation on preribosomes and fractional rRNA 2'-O-methylation by such snoRNA/Ps suggest that they do not act in a strictly defined order. A growing body of evidence supports the step-wise assembly and compaction of different pre-ribosomal regions in eukaryotes (4), analogous to the hierarchical model of bacterial ribosome assembly. While the stochastic pre-ribosomeassociation of snoRNAs with overlapping basepairing sites in the same domain is accommodated within this framework, it is likely that snoRNPs are broadly recruited to preribosomes in the order in which their target sites are transcribed and that snoRNAs guiding modifications in different domains of the ribosome that compact at different stages instead act sequentially. 


\section{DATA AVAILABILITY}

GEO database: identifier GSE155720.

\section{FUNDING}

This work was supported by the Deutsche Forschungsgemeinschaft (SFB860 to M.T.B and K.E.B), the University Medical Centre Göttingen (to M.T.B. and K.E.B), the Biotechnology and Biological Sciences Research Council/Medical Research Council (UK) (BB/R00143X/1 to N.J.W and C.S.), the Wellcome Trust (092076 to N.J.W) and the Royal Society (UK) (UF150691 to C.S.), the EU COST Action CA16120 (STSM41493 to G.R.A and M.T.B.), the Danish Research Council for Independent Research (DFF-4183-00486 to H.N.), the Lundbeck Foundation (R198-2015-174 to H.N.) and the Danish Cancer Society (R167-A10943-17-S2 to N.K.).

\section{ACKNOWLEDGEMENTS}

We thank Anne-Caren Zeiss, Nidhi Sharma and Isabela Magierowska for molecular cloning and preparation of yeast strains, and Dr. Grzegorz Kudla for help with CLASH analysis.

\section{CONFLICT OF INTEREST STATEMENT}

None declared. 


\section{REFERENCES}

1. Ben-Shem,A., Garreau de Loubresse,N., Melnikov,S., Jenner,L., Yusupova,G. and Yusupov,M. (2011) SOM: The structure of the eukaryotic ribosome at $3.0 \AA$ resolution. Science, 334, 1524-9.

2. Warner,J.R. (1999) The economics of ribosome biosynthesis in yeast. Trends Biochem. Sci., 24, 437-440.

3. Anger,A.M., Armache,J.-P., Berninghausen,O., Habeck,M., Subklewe,M., Wilson,D.N. and Beckmann,R. (2013) Structures of the human and Drosophila $80 S$ ribosome. Nature, 497, 80-85.

4. Klinge,S. and Woolford,J.L.J. (2019) Ribosome assembly coming into focus. Nat. Rev. Mol. Cell Biol., 20, 116-131.

5. Bohnsack,K.E. and Bohnsack,M.T. (2019) Uncovering the assembly pathway of human ribosomes and its emerging links to disease. EMBO J., 10.15252/embj.2018100278.

6. Henras,A.K., Plisson-Chastang,C., O'Donohue,M.-F., Chakraborty,A. and Gleizes,P.-E. (2015) An overview of pre-ribosomal RNA processing in eukaryotes. Wiley Interdiscip. Rev. RNA, 6, 225-242.

7. Koš,M. and Tollervey,D. (2010) Yeast Pre-rRNA Processing and Modification Occur Cotranscriptionally. Mol. Cell, 37, 809-820.

8. Birkedal,U., Christensen-Dalsgaard,M., Krogh,N., Sabarinathan,R., Gorodkin,J. and Nielsen,H. (2015) Profiling of ribose methylations in RNA by high-throughput sequencing. Angew. Chemie - Int. Ed., 54, 451-455.

9. Watkins,N.J. and Bohnsack,M.T. (2012) The box C/D and H/ACA snoRNPs: Key players in the modification, processing and the dynamic folding of ribosomal RNA. Wiley Interdiscip. Rev. RNA, 3, 397-414.

10. Sloan,K.E., Warda,A.S., Sharma,S., Entian,K.-D., Lafontaine,D.L.J. and Bohnsack,M.T. (2017) Tuning the ribosome: The influence of rRNA modification on eukaryotic ribosome biogenesis and function. RNA Biol., 14, 1138-1152.

11. van Nues,R.W., Granneman,S., Kudla,G., Sloan,K.E., Chicken,M., Tollervey,D. and Watkins,N.J. (2011) Box C/D snoRNP catalysed methylation is aided by additional prerRNA base-pairing. EMBO J., 30, 2420-30.

12. Decatur,W.A. and Fournier,M.J. (2002) rRNA modifications and ribosome function. Trends Biochem. Sci., 27, 344-351.

13. Sharma,S. and Lafontaine,D.L.J. (2015) 'View From A Bridge': A New Perspective on Eukaryotic rRNA Base Modification. Trends Biochem. Sci., 40, 560-575.

14. Bachellerie,J.P. and Cavaillé,J. (1997) Guiding ribose methylation of rRNA. Trends Biochem. Sci., 22, 257-261.

15. Krogh,N., Jansson,M.D., Hafner,S.J., Tehler,D., Birkedal,U., Christensen-Dalsgaard,M., 
Lund,A.H. and Nielsen,H. (2016) Profiling of 2'-O-Me in human rRNA reveals a subset of fractionally modified positions and provides evidence for ribosome heterogeneity. Nucleic Acids Res., 44, 7884-7895.

16. Marchand,V., Blanloeil-Oillo,F., Helm,M. and Motorin,Y. (2016) Illumina-based RiboMethSeq approach for mapping of 2???-O-Me residues in RNA. Nucleic Acids Res., 44, 1-12.

17. Hebras,J., Krogh,N., Marty,V., Nielsen,H. and Cavaille,J. (2020) Developmental changes of rRNA ribose methylations in the mouse. RNA Biol., 17, 150-164.

18. Barandun,J., Chaker-Margot,M., Hunziker,M., Molloy,K.R., Chait,B.T. and Klinge,S. (2017) The complete structure of the small-subunit processome. Nat. Struct. Mol. Biol., 10.1038/nsmb.3472.

19. Sloan,K.E., Gleizes,P.-E. and Bohnsack,M.T. (2016) Nucleocytoplasmic Transport of RNAs and RNA-Protein Complexes. J. Mol. Biol., 428, 2040-2059.

20. Gerhardy,S., Menet,A.M., Pena,C., Petkowski,J.J. and Panse,V.G. (2014) Assembly and nuclear export of pre-ribosomal particles in budding yeast. Chromosoma, 123, 327-344. 21. Kressler,D., Hurt,E. and Bassler,J. (2010) Driving ribosome assembly. Biochim. Biophys. Acta, 1803, 673-683.

22. Prattes,M., Lo,Y.-H., Bergler,H. and Stanley,R.E. (2019) Shaping the Nascent Ribosome: AAA-ATPases in Eukaryotic Ribosome Biogenesis. Biomolecules, 9.

23. Martin,R., Straub,A.U., Doebele,C. and Bohnsack,M.T. (2013) DExD/H-box RNA helicases in ribosome biogenesis. RNA Biol., 10, 4-18.

24. Sloan,K.E. and Bohnsack,M.T. (2018) Unravelling the Mechanisms of RNA Helicase Regulation. Trends Biochem. Sci., 43, 237-250.

25. Dembowski,J.A., Kuo,B. and Woolford,J.L. (2013) Has1 regulates consecutive maturation and processing steps for assembly of $60 \mathrm{~S}$ ribosomal subunits. Nucleic Acids Res., 41, 7889-7904.

26. Khoshnevis,S., Askenasy,I., Johnson,M.C., Dattolo,M.D., Young-Erdos,C.L., Stroupe,M.E. and Karbstein,K. (2016) The DEAD-box Protein Rok1 Orchestrates 40S and 60S Ribosome Assembly by Promoting the Release of Rrp5 from Pre-40S Ribosomes to Allow for 60S Maturation. PLoS Biol., 14, 1-25.

27. Bruning,L., Hackert,P., Martin,R., Davila Gallesio,J., Aquino,G.R.R., Urlaub,H., Sloan,K.E. and Bohnsack,M.T. (2018) RNA helicases mediate structural transitions and compositional changes in pre-ribosomal complexes. Nat. Commun., 9, 5383.

28. Bohnsack,M.T., Martin,R., Granneman,S., Ruprecht,M., Schleiff,E. and Tollervey,D. (2009) Prp43 Bound at Different Sites on the Pre-rRNA Performs Distinct Functions in Ribosome Synthesis. Mol. Cell, 36, 583-592.

29. Leeds,N.B., Small,E.C., Hiley,S.L., Hughes,T.R. and Staley,J.P. (2006) The splicing 
factor Prp43p, a DEAH box ATPase, functions in ribosome biogenesis. Mol. Cell. Biol., 26, 513-22.

30. Liang,X.-H. and Fournier,M.J. (2006) The helicase Has1p is required for snoRNA release from pre-rRNA. Mol. Cell. Biol., 26, 7437-50.

31. Bohnsack,M.T., Kos,M. and Tollervey,D. (2008) Quantitative analysis of snoRNA association with pre-ribosomes and release of snR30 by Rok1 helicase. EMBO Rep., $\mathbf{9}$, 1230-6.

32. Martin,R., Hackert,P., Ruprecht,M., Simm,S., Bruning,L., Mirus,O., Sloan,K.E., Kudla,G., Schleiff,E. and Bohnsack,M.T. (2014) A pre-ribosomal RNA interaction network involving snoRNAs and the Rok1 helicase. RNA, 20, 1173-1182.

33. Sardana,R., Liu,X., Granneman,S., Zhu,J., Gill,M., Papoulas,O., Marcotte,E.M., Tollervey,D., Correll,C.C. and Johnson,A.W. (2015) The DEAH-box helicase Dhr1 dissociates U3 from the pre-rRNA to promote formation of the central pseudoknot. PLoS Biol., 13, e1002083.

34. van Nues,R.W. and Watkins,N.J. (2016) Unusual C'/D' motifs enable box C/D snoRNPs to modify multiple sites in the same rRNA target region. Nucleic Acids Res., 10.1093/nar/gkw842.

35. Kiianitsa,K., Solinger,J.A. and Heyer,W.-D. (2003) NADH-coupled microplate photometric assay for kinetic studies of ATP-hydrolyzing enzymes with low and high specific activities. Anal. Biochem., 321, 266-271.

36. Choudhury,P., Hackert,P., Memet,I., Sloan,K.E. and Bohnsack,M.T. (2019) The human RNA helicase DHX37 is required for release of the U3 snoRNP from pre-ribosomal particles. RNA Biol., 16, 54-68.

37. Sloan,K.E., Leisegang,M.S., Doebele,C., Ramírez,A.S., Simm,S., Safferthal,C., Kretschmer,J., Schorge,T., Markoutsa,S., Haag,S., et al. (2015) The association of lateacting snoRNPs with human pre-ribosomal complexes requires the RNA helicase DDX21. Nucleic Acids Res., 43, 553-564.

38. Memet,I., Doebele,C., Sloan,K.E. and Bohnsack,M.T. (2017) The G-patch protein NFkappaB-repressing factor mediates the recruitment of the exonuclease XRN2 and activation of the RNA helicase DHX15 in human ribosome biogenesis. Nucleic Acids Res., 45, 5359-5374.

39. Krogh,N., Birkedal,U. and Nielsen,H. (2017) RiboMeth-seq: Profiling of 2'-O-Me in RNA. Methods Mol. Biol., 1562, 189-209.

40. Krogh,N., Kongsbak-Wismann,M., Geisler,C. and Nielsen,H. (2017) Substoichiometric ribose methylations in spliceosomal snRNAs. Org. Biomol. Chem., 15, 8872-8876.

41. Yu,Y.T., Shu,M.D. and Steitz,J.A. (1997) A new method for detecting sites of 2'-Omethylation in RNA molecules. RNA, 3, 324-331. 
42. Travis,A.J., Moody,J., Helwak,A., Tollervey,D. and Kudla,G. (2014) Hyb: a bioinformatics pipeline for the analysis of CLASH (crosslinking, ligation and sequencing of hybrids) data. Methods, 65, 263-273.

43. Weaver,P.L., Sun,C. and Chang,T.H. (1997) Dbp3p, a putative RNA helicase in Saccharomyces cerevisiae, is required for efficient pre-rRNA processing predominantly at site A3. Mol. Cell. Biol., 17, 1354-1365.

44. Garcia,I., Albring,M.J. and Uhlenbeck,O.C. (2012) Duplex destabilization by four ribosomal DEAD-box proteins. Biochemistry, 51, 10109-10118.

45. Breslow,D.K., Cameron,D.M., Collins,S.R., Schuldiner,M., Stewart-Ornstein,J., Newman,H.W., Braun,S., Madhani,H.D., Krogan,N.J. and Weissman,J.S. (2008) A comprehensive strategy enabling high-resolution functional analysis of the yeast genome. Nat. Methods, 5, 711-718.

46. Wu,S., Tutuncuoglu,B., Yan,K., Brown,H., Zhang,Y., Tan,D., Gamalinda,M., Yuan,Y., Li,Z., Jakovljevic,J., et al. (2016) Diverse roles of assembly factors revealed by structures of late nuclear pre-60S ribosomes. Nature, 534, 133-137.

47. Petfalski,E., Dandekar,T., Henry,Y. and Tollervey,D. (1998) Processing of the precursors to small nucleolar RNAs and rRNAs requires common components. Mol. Cell. Biol., 18, 1181-1189.

48. Giorgi,C., Fatica,A., Nagel,R. and Bozzoni,I. (2001) Release of U18 snoRNA from its host intron requires interaction of Nop1p with the Rnt1p endonuclease. EMBO J., 20, 6856-6865.

49. Allmang,C., Kufel,J., Chanfreau,G., Mitchell,P., Petfalski,E. and Tollervey,D. (1999) Functions of the exosome in rRNA, snoRNA and snRNA synthesis. EMBO J., 18, 53995410.

50. Ooi,S.L., Samarsky,D.A., Fournier,M.J. and Boeke,J.D. (1998) Intronic snoRNA biosynthesis in Saccharomyces cerevisiae depends on the lariat-debranching enzyme: intron length effects and activity of a precursor snoRNA. RNA, 4, 1096-1110.

51. Kiss-László,Z., Henry,Y., Bachellerie,J.P., Caizergues-Ferrer,M. and Kiss,T. (1996) Sitespecific ribose methylation of preribosomal RNA: A novel function for small nucleolar RNAs. Cell, 85, 1077-1088.

52. Petrov,A.S., Bernier,C.R., Gulen,B., Waterbury,C.C., Hershkovits,E., Hsiao,C., Harvey,S.C., Hud,N. V, Fox,G.E., Wartell,R.M., et al. (2014) Secondary structures of rRNAs from all three domains of life. PLoS One, 9, e88222.

53. Guglielmi,B. and Werner,M. (2002) The yeast homolog of human PinX1 is involved in rRNA and small nucleolar RNA maturation, not in telomere elongation inhibition. J. Biol. Chem., 277, 35712-35719.

54. Hamann,F., Enders,M. and Ficner,R. (2019) Structural basis for RNA translocation by 
DEAH-box ATPases. Nucleic Acids Res., 47, 4349-4362.

55. Ozgur,S., Buchwald,G., Falk,S., Chakrabarti,S., Prabu,J.R. and Conti,E. (2015) The conformational plasticity of eukaryotic RNA-dependent ATPases. FEBS J., 282, 850863.

56. Martin,R., Hackert,P., Ruprecht,M., Simm,S., Brüning,L., Mirus,O., Sloan,K.E., Kudla,G. and Schleiff,E. (2014) A pre-ribosomal RNA interaction network involving snoRNAs and the A pre-ribosomal RNA interaction network involving snoRNAs and the Rok1 helicase. Rna, 20, 1-10.

57. Bohnsack,M.T., Tollervey,D. and Granneman,S. (2012) Identification of RNA helicase target sites by UV cross-linking and analysis of cDNA 1st ed. Elsevier Inc.

58. Cherry,J.M., Hong,E.L., Amundsen,C., Balakrishnan,R., Binkley,G., Chan,E.T., Christie,K.R., Costanzo,M.C., Dwight,S.S., Engel,S.R., et al. (2012) Saccharomyces Genome Database: the genomics resource of budding yeast. Nucleic Acids Res., 40, D700-5.

59. Leontis,N.B. and Westhof,E. (2001) Geometric nomenclature and classification of RNA base pairs. $R N A, 7,499-512$. 


\section{FIGURE LEGENDS}

Figure 1. The putative RNA helicase Dbp3 is an RNA-dependent ATPase. (A) Schematic view of Dbp3 showing the amino acid boundaries of the RecA1 and RecA2 domains as well as the amino acid substitution made within the evolutionarily conserved DEAD motif. (B) $\mathrm{N}$ terminally $\mathrm{His}_{10}-\mathrm{ZZ}$ tagged wild type Dbp3 (Dbp3 $\mathrm{wT}_{\mathrm{T}}$ ) and an equivalent protein carrying a glutamate to glutamine substitution at position 263 (Dbp3 ${ }_{\mathrm{E} 263 \mathrm{Q}}$ ) were recombinantly expressed in $E$. coli and purified by $\mathrm{Ni}^{2+}$-affinity chromatography. Purified proteins were separated by SDS-PAGE and visualised by Coomassie staining. (C) In vitro NADH-coupled ATPase assays were used to monitor ATP hydrolysis by Dbp3wt and Dbp3 ${ }_{\mathrm{E} 263 \mathrm{Q}}$ in the presence of increasing amounts of RNA. A sample containing no protein was included as a control for background hydrolysis of ATP. The data represent the mean of three independent experiments \pm standard deviation. ${ }^{* * *}=p<0.001,{ }^{* * * *}=p<0.0001$, n.s. $=$ non-significant.

Figure 2. The catalytic activity of Dbp3 is required for biogenesis of the large ribosomal subunit. (A) Schematic view of the major pre-rRNA processing intermediates present in yeast. Black rectangles indicate mature rRNA sequences and black lines represent internal transcribed spacers (ITS1 and ITS2) and external transcribed spacers (5' ETS and 3' ETS). The positions of selected pre-rRNA cleavage sites are marked on the 35S pre-rRNA transcript. A magnified view of the ITS1 and ITS2 regions with major pre-rRNA cleavage sites and the hybridisation positions of probes used for northern blotting (indicated by red lines) is shown below the 35S transcript. (B) Wild type yeast (WT) or a strain lacking Dbp3 ( $\Delta d b p 3)$ were transformed with an empty pRS415 vector (EV) or plasmids for the expression of Dbp3wT or $\mathrm{Dbp3}_{\mathrm{E} 263 \mathrm{Q}}$ from the endogenous $\mathrm{DBP} 3$ promoter. Pre-rRNA levels were analysed by northern blotting using a mixture of probes hybridising within ITS1 or ITS2. Mature rRNAs were visualised by methylene blue staining. The ratio of $27 S_{A} / 27 S_{B}$ was calculated in three independent experiments and is shown as mean \pm standard deviation. Significance was calculated using a Student's t-test $\left({ }^{* *}=p<0.01,{ }^{* \star *}=p<0.001\right.$, n.s. $=$ non-significant). (C) PrerRNA processing in wild type yeast (WT), cells lacking Dbp3 ( $\Delta d b p 3)$, cells expressing reduced levels of Nop56 (NOP56 $\left.{ }_{D A m P}\right)$ or both ( $\left.\triangle d b p 3-N O P 56_{D A m P}\right)$ was analysed as in $(\mathrm{B})$.

Figure 3. Dbp3 is required for 2'-O-methylation of specific rRNA nucleotides. (A-B) RiboMeth-seq analysis was performed on wild type yeast (WT) or a strain lacking Dbp3 $(\Delta d b p 3)$. RiboMeth-seq scores, indicating the fraction of methylation, are plotted for each 2'O-methylated nucleotide in the 18S rRNA (A) and the 25S rRNA (B). The data shown are the mean of two biological replicates and error bars represent standard deviation. The snoRNAs that guide each modification are indicated in blue and modifications for which the RMS score is decreased by $>10 \%$ in the absence of Dbp3 are highlighted in red. (C) Dbp3-dependent 
(red) and Dbp3-independent (black) 2'-O-methylations are mapped onto the tertiary structure of a pre-60S complex purified via Nog2 (PBD: 3JCT) (46). (D-F) Wild type yeast cells or a strain lacking Dbp3 were transformed with an empty pRS415 vector (EV) or plasmids for the expression of Dbp3 $\mathrm{wT}_{\mathrm{w}}$ or $\mathrm{Dbp} 3_{\mathrm{E} 263 \mathrm{Q}}$ from the endogenous $D B P 3$ promoter. RNA was isolated from these strains and RNase $H(R H)$-mediated cleavage assays targeting Am876 (snR72), Um898 (snR40) or Um2724 (snR67) were performed, and full length 25S rRNA and specific cleavage products (indicated by arrow heads) were detected by northern blotting using probes hybridising up- and down-stream of the cleavage sites ( $D$ and $E$ ) or only downstream of the cleavage site $(F)$. Asterisks indicate non-specific cleavage products - note that the extent of non-specific RNase H-mediated cleavage is reduced upon cleavage at a specific cleavage site.

Figure 4. Maturation of the intron-encoded U18 and U24 snoRNAs requires Dbp3. (A) Total RNA from wild type yeast (WT), and $\Delta d b p 3$ and $4 r r p 6$ strains was separated by denaturing PAGE and analysed by northern blotting using probes hybridising to the U18 and U24 snoRNAs. (B and $\mathbf{C}$ ) RMS sequencing reads derived from wild type yeast or the $\Delta d b p 3$ strain were mapped to the annotated U18 sequence +/- $50 \mathrm{nt}$, and after normalisation for expression level, the numbers of reads mapping to each nucleotide were determined. Profiles for the 5 ' end (B) and 3' end (C) are shown. Nucleotides of box $C(B)$ and box $D(C)$ are underlined and the annotated 5' and 3' ends (58) are indicated. (D) Schematic view of the secondary structure of the pre-U18 snoRNA with key features indicated. Basepairing is shown according to (59) (E) Total RNA from wild type yeast (WT) or a strain lacking Dbp3 ( $\Delta d b p 3)$ carrying an empty pRS415 vector (EV) or plasmids for the expression of Dbp3 ${ }_{w T}$ or Dbp3 $3_{E 263 Q}$ from the endogenous $D B P 3$ promoter was analysed as in $(A)$. (F) Extracts from yeast expressing Nop1-His-Tev protease cleavage site-ProtA (HTP) in the wild type or $\Delta d b p 3$ backgrounds were used for pulldown assays on IgG sepharose. Co-purified RNAs were analysed as in (A). (G) Whole cell extracts prepared from wild type yeast or the $\Delta d b p 3$ strain were separated by sucrose density gradient centrifugation. RNA from individual fractions was separated by denaturing PAGE and analysed by northern blotting using probes to the snoRNAs indicated to the right. The fractions containing "free" snoRNAs and pre-ribosomeassociated snoRNAs are indicated.

Figure 5. A subset of box C/D snoRNAs accumulate on pre-ribosomes when Dbp3 is lacking. Extracts were separated by sucrose density gradient centrifugation as in (D). Fractions containing either (pre-)ribosomal complexes or non-ribosome-associated snoRNPs were pooled and RNA was extracted. Polyadenylation and reverse transcription were performed and the levels of each of the 75 yeast snoRNAs in each sample was determined by 
RT-qPCR. The relative distribution of each box C/D snoRNA between (pre-)ribosome-bound and non-ribosome-associated fractions was calculated and differences in this ratio between the wild type and $\Delta d b p 3$ strains are shown graphically. Three independent experiments were performed and the data are presented as mean \pm standard deviation. Dashed red lines indicate the upper and low thresholds for significant accumulation and exclusion of snoRNAs on/from pre-ribosomes respectively.

Figure 6. Many snoRNAs that accumulate on pre-ribosomes in the absence of Dbp3 and guide Dbp3-dependent rRNA 2'-O-methylations have overlapping pre-rRNA basepairing sites. (A-E) 2'-O-methylations reduced in $\triangle d b p 3$ and the snoRNAs that guide them are highlighted on the secondary structure of the 25S rRNA (52) in red and the pre-rRNA nucleotides involved in basepairing interactions with the guiding snoRNAs are indicated in green. Extra snoRNA basepairing is shown in blue. Magnified views of selected areas of interest from domain I (A), domain V (B), domain $0(C)$, domain IV (D) and domain V (E) are shown. (F) Whole cell extracts prepared from wild type yeast were separated by sucrose density gradient centrifugation. RNA from individual fractions was separated by denaturing PAGE and analysed by northern blotting using probes to the snoRNAs indicated to the right. The fractions containing "free" snoRNAs and pre-ribosome-associated snoRNAs are indicated.

Figure 7. Overexpression of snR67 rescues 2'-O-methylation of Gm2619 and Um2724 when Dbp3 is lacking. (A-B) RNA from wild type yeast or strains lacking Dbp3 transformed with an empty pRS416 vector (EV) or a plasmid for the overexpression of snR67 from a $p G A L 1$ promoter (snR67 $\mathrm{OE})$, or a strain in which $S N R 67$ was deleted $(\Delta 67)$ was subjected to RNase $\mathrm{H}$ $(\mathrm{RH})$-mediated cleavage targeting 25S-Gm2619 (A) or 25S-Um2724 (B). Full length 25S rRNA and cleavage products (indicated by arrow head) were detected by northern blotting using probes hybridising downstream of the cleavage sites. Asterisks indicate non-specific cleavage products - note that the extent of non-specific RNase $\mathrm{H}$-mediated cleavage is reduced upon cleavage at a specific cleavage site.

Figure 8. Prp43 is required for efficient rRNA 2'-O-methylation and crosslinks to snoRNA-pre-rRNA duplexes. (A) Proteins from wild type yeast (WT) and the pGALs-HAPrp43 strain grown in galactose-containing media were separated by SDS-PAGE. Western blotting was performed using antibodies against Prp43 and, as a loading control, Pgk1. (B) The pGALs-HA-Prp43 strain was grown in exponential phase in media containing galactose (Gal) or glucose (Glu) for $12 \mathrm{~h}$. Cells were harvested at the indicated time points, and proteins were extracted, separated by SDS-PAGE and analysed by western blotting using antibodies against the HA tag and Pgk1. (C-D) RiboMeth-seq analysis was performed on RNA derived 
from the pGALs-HA-Prp43 strain grown exponentially in media containing galactose (Gal) or glucose (Glu) for $8 \mathrm{~h}$. RiboMeth-seq scores, indicating the fraction of methylation, are plotted for each 2'-O-methylated nucleotide in the 18S rRNA (C) and the 25S rRNA (D). The data shown are the mean of two biological replicates and error bars represent standard deviation. The snoRNAs that guide each modification are indicated in blue and modifications for which the RMS score is decreased by $>10 \%$ when Prp43 is depleted (Glu) are highlighted in red. (E) The positions of predicted snoRNA basepairing sites (light blue) and chimeric reads identified in the Prp43 (upper) and WT (lower) CRAC datasets [26] by Hyb (dark blue) are mapped according to their positions on the 25S rRNA sequence. Sites of overlap between snoRNA basepairing sites and CLASH hybrids are shown in red. The profile of Prp43 crosslinking on the 25S rRNA sequence (28) is shown in grey with the peak heights given on the right. 

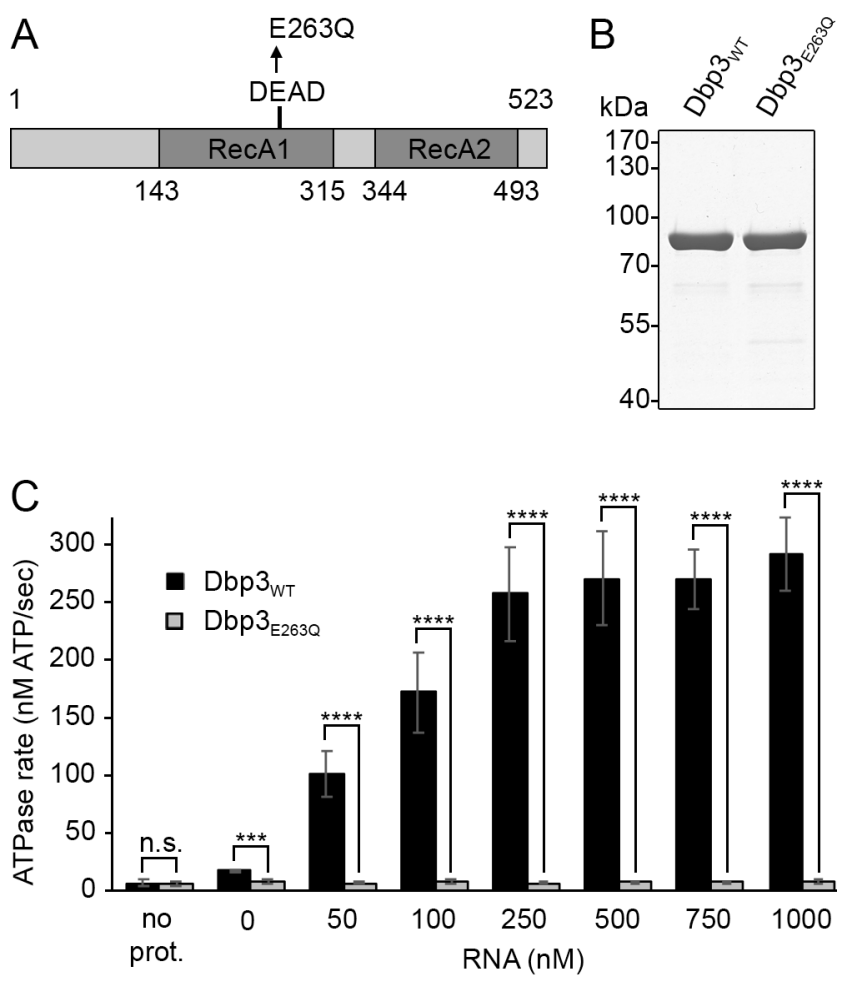

Aquino et al., Figure 1 

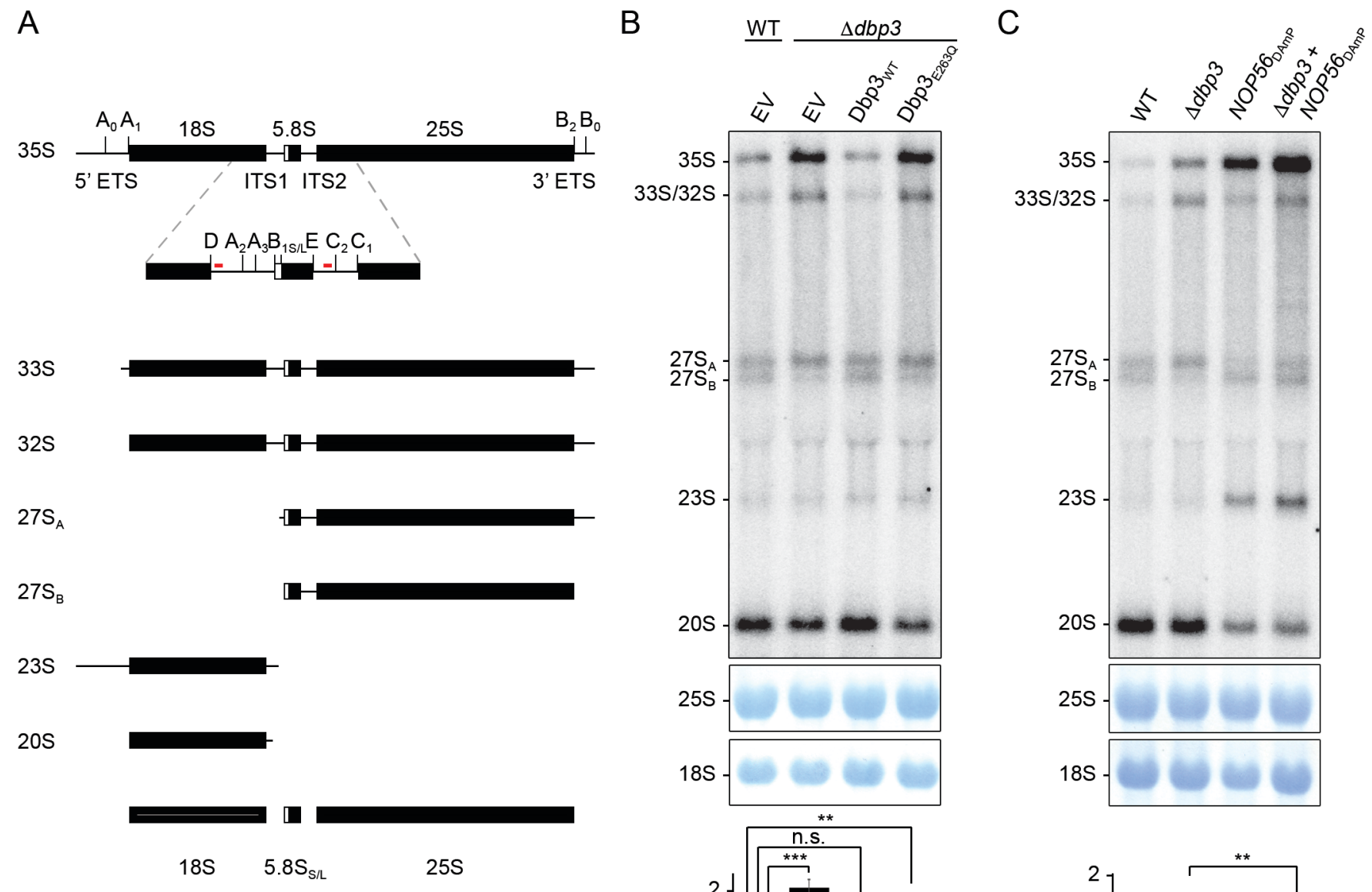

$18 \mathrm{~S} \quad 5.8 \mathrm{~S}_{\mathrm{S} / \mathrm{L}}$
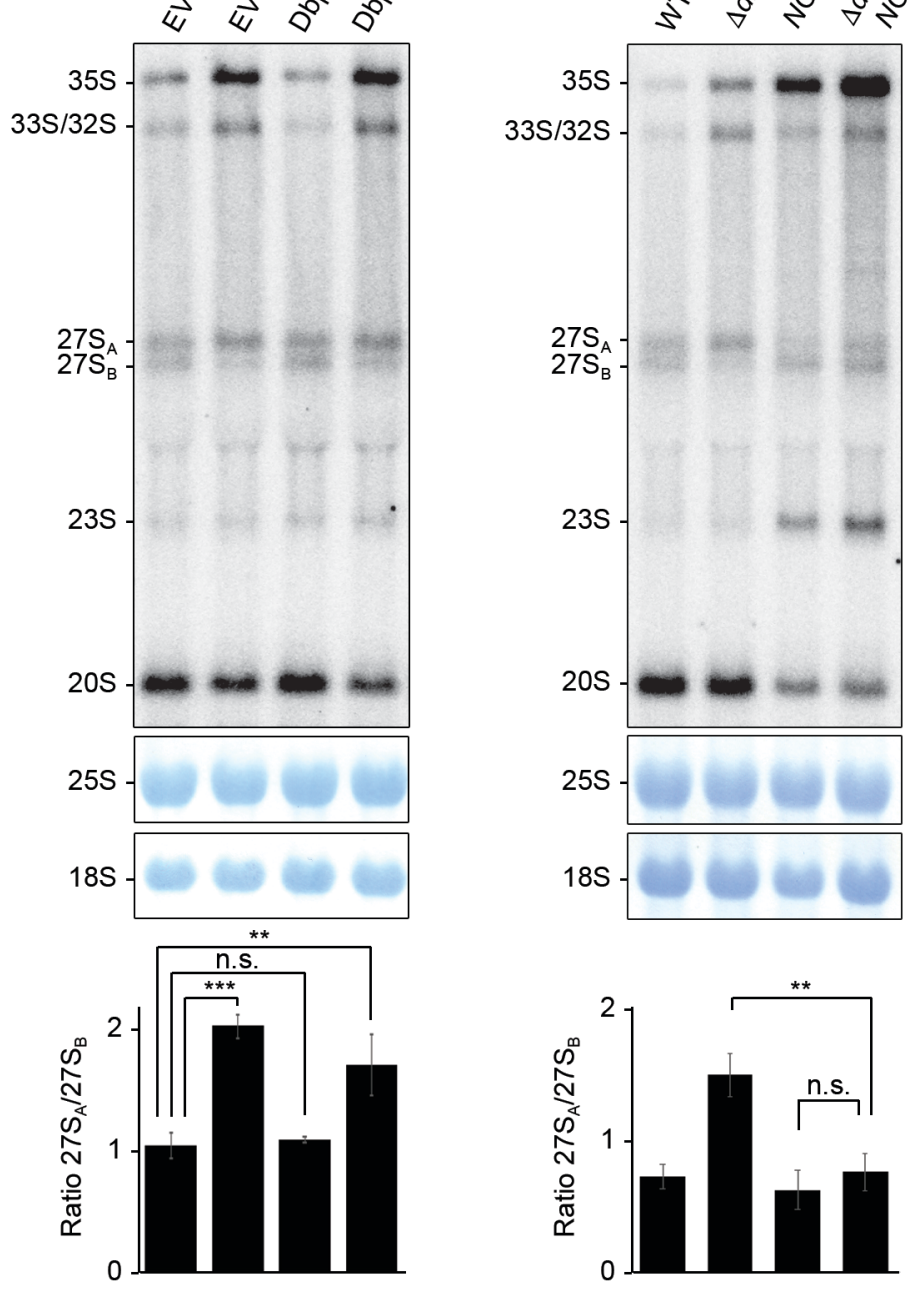

Aquino et al., Figure 2 

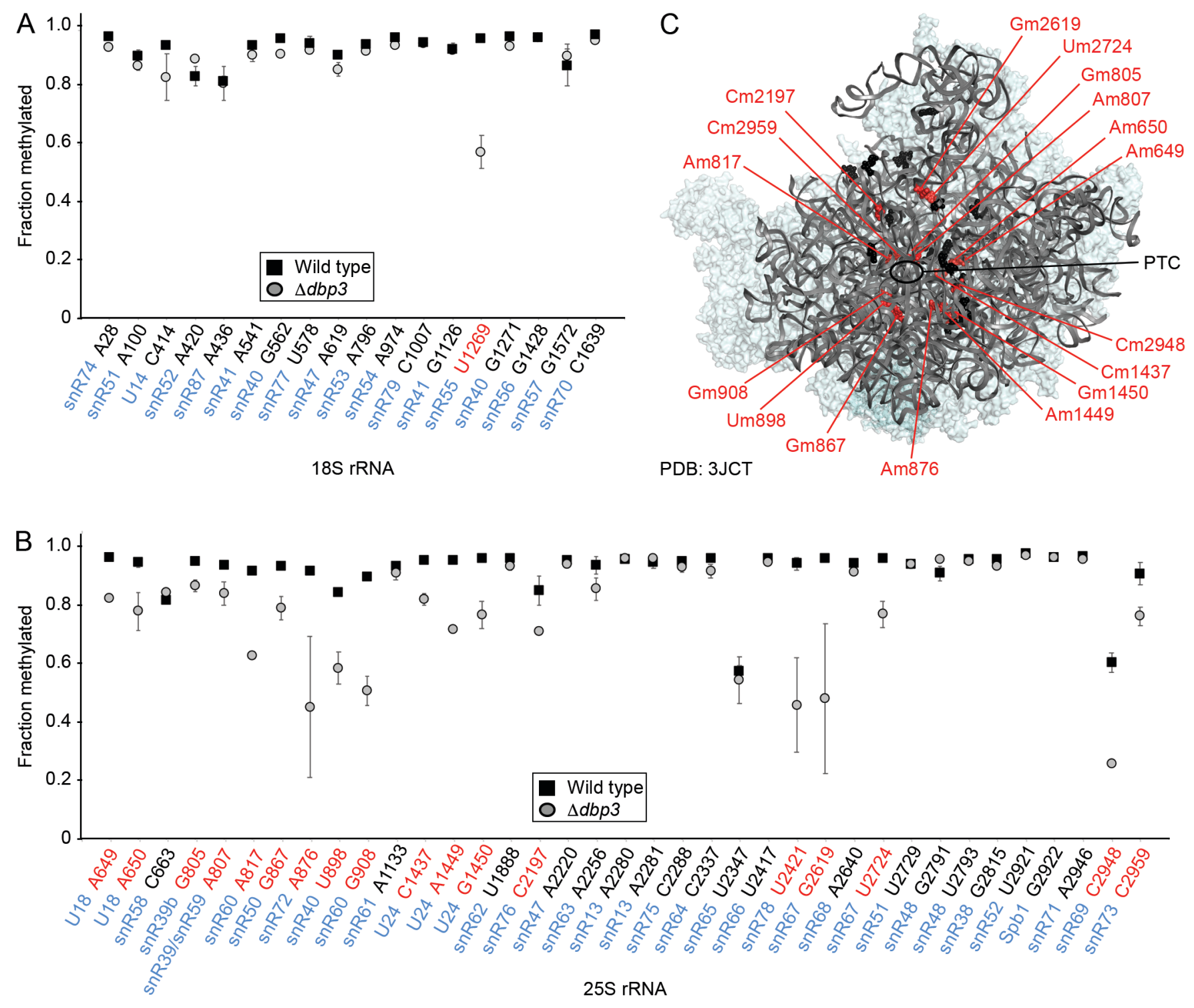

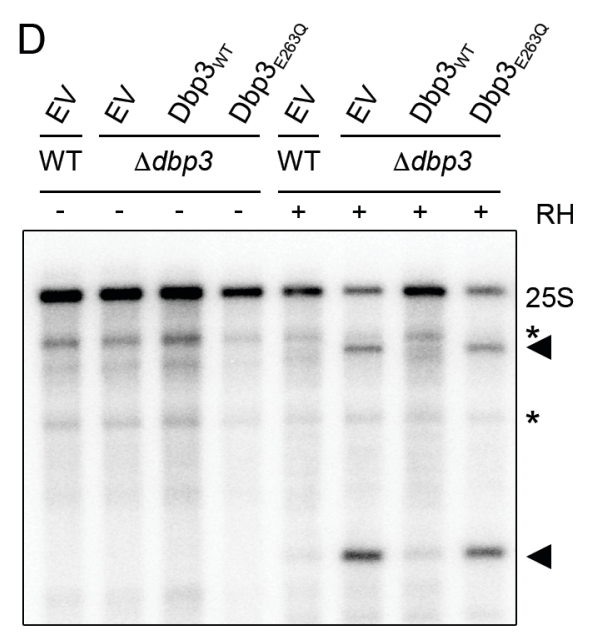

Am876

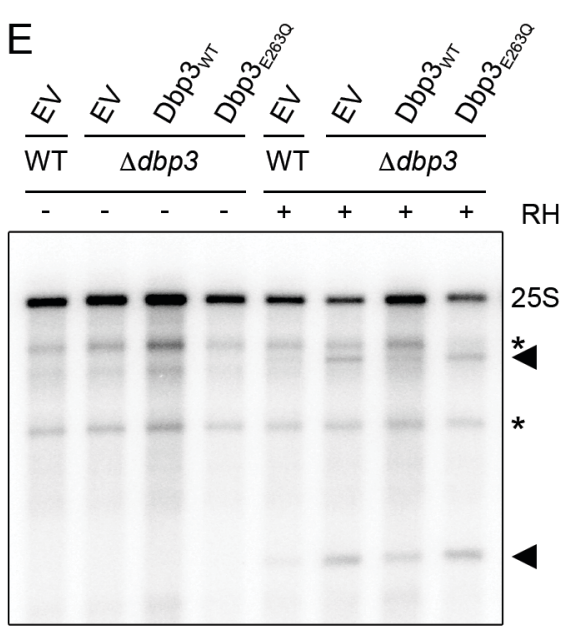

Um898

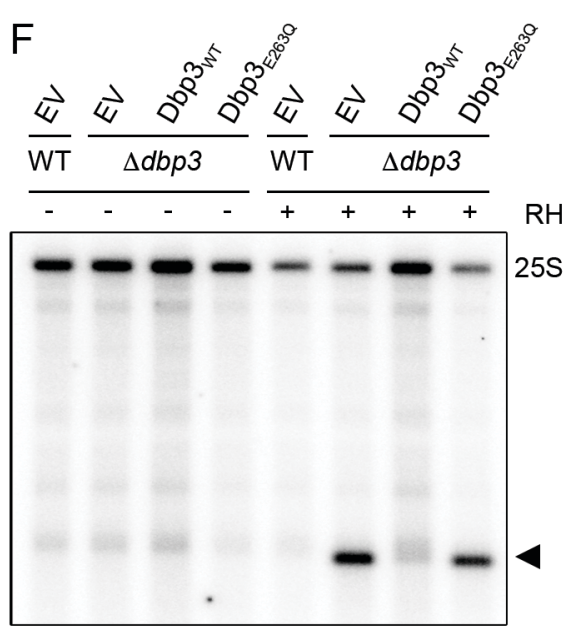

Um2724

Aquino et al., Figure 3 

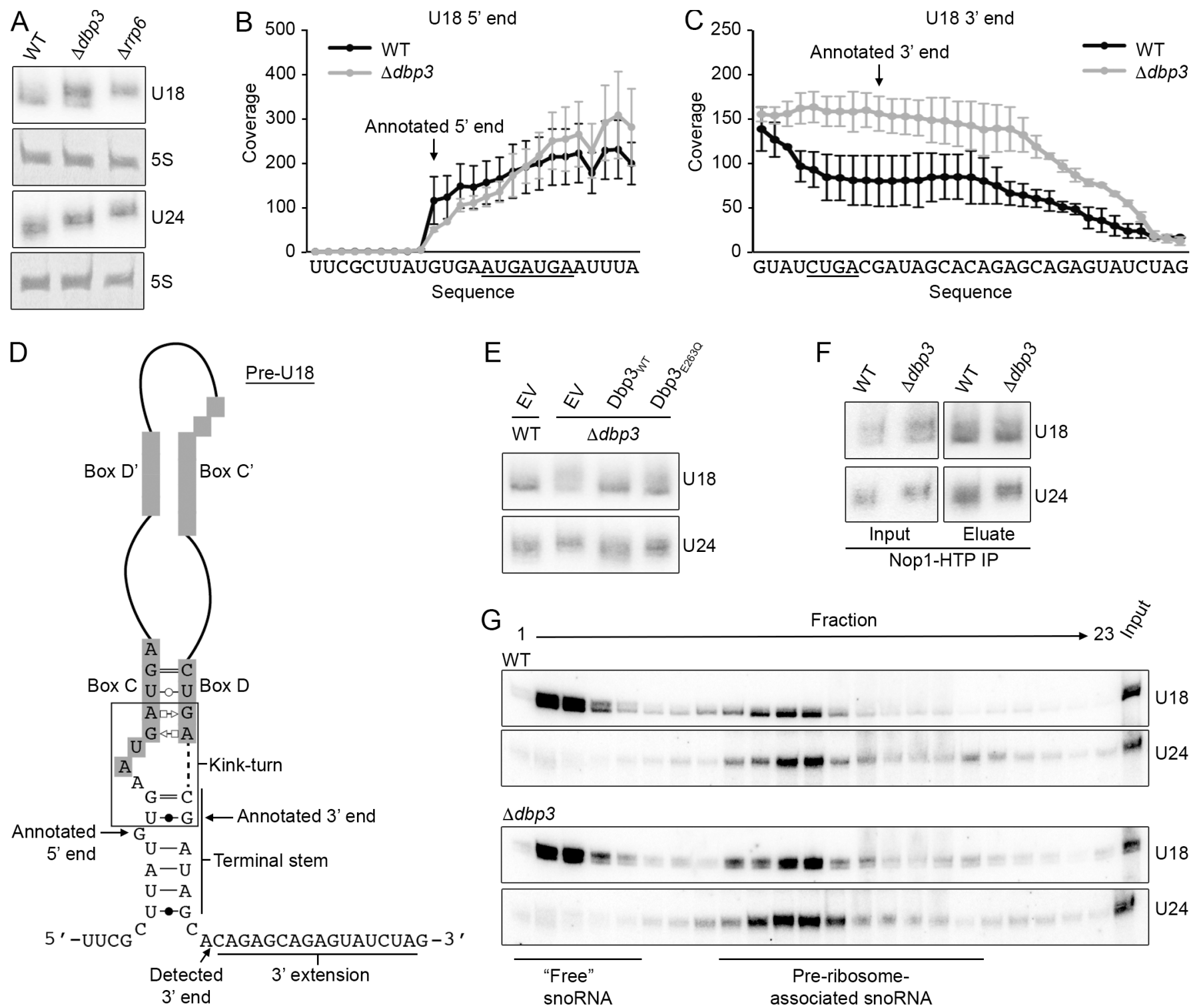

Aquino et al., Figure 4 


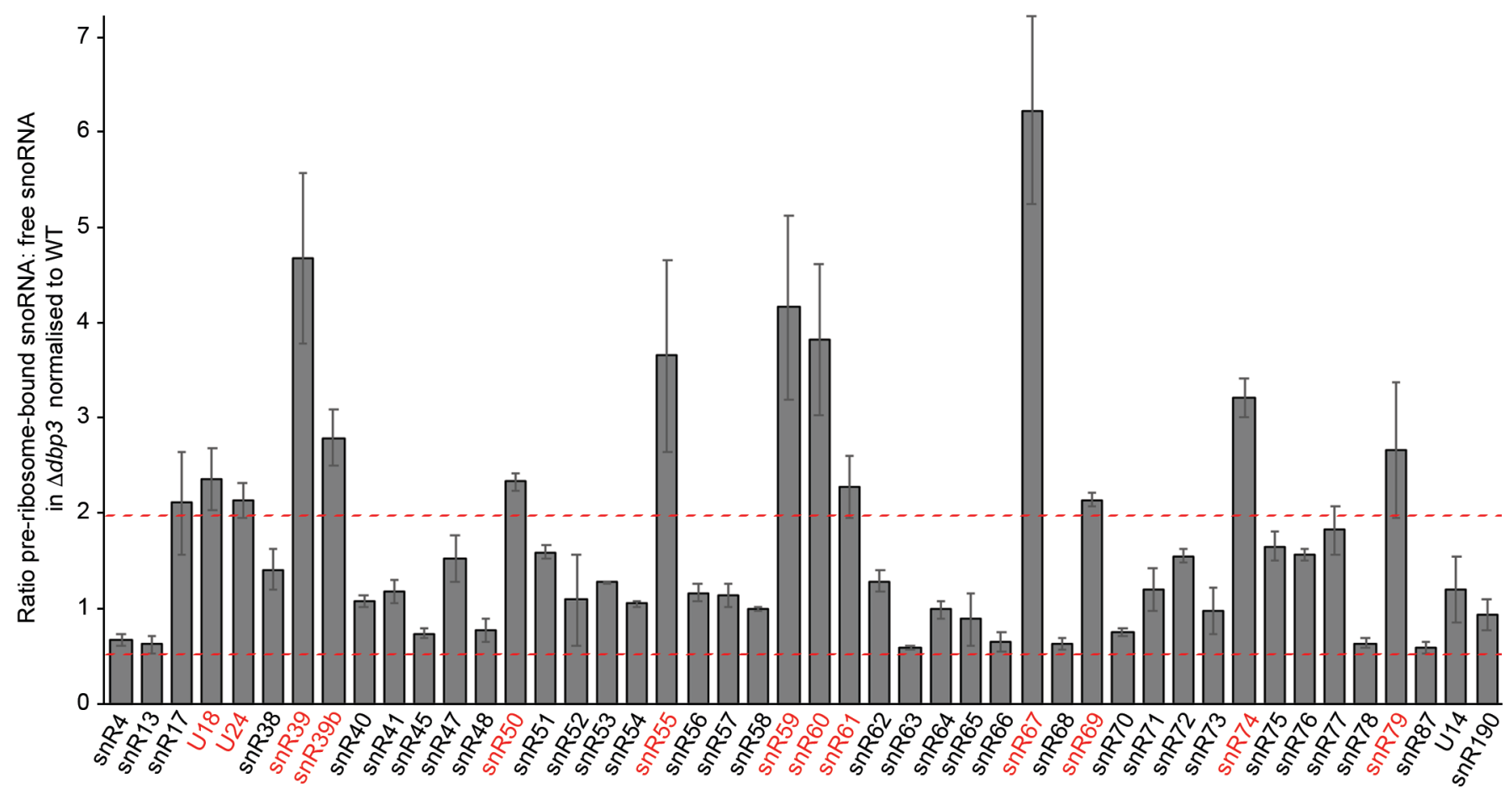

Aquino et al., Figure 5 

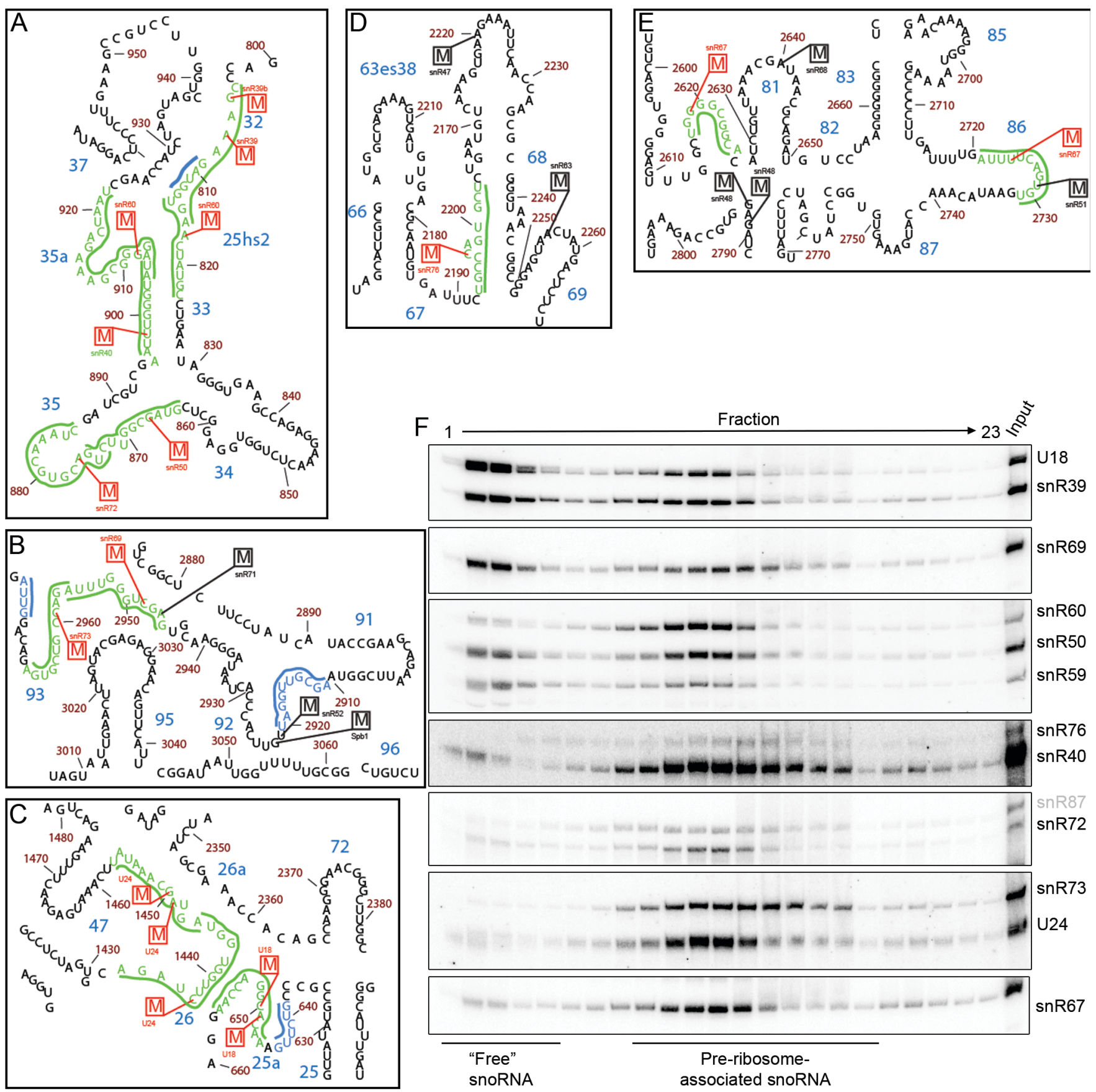

Aquino et al., Figure 6 

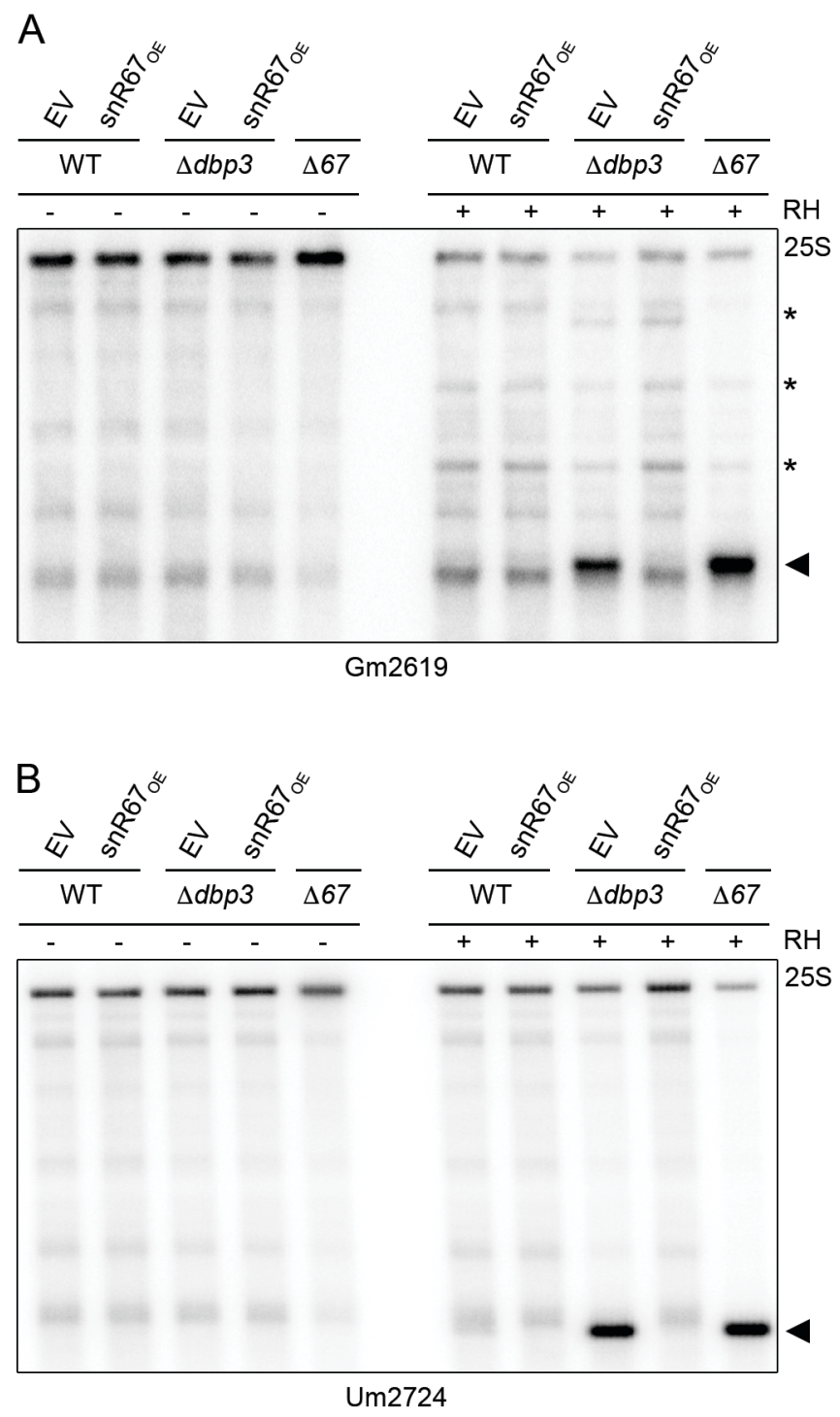

Aquino et al., Figure 7 

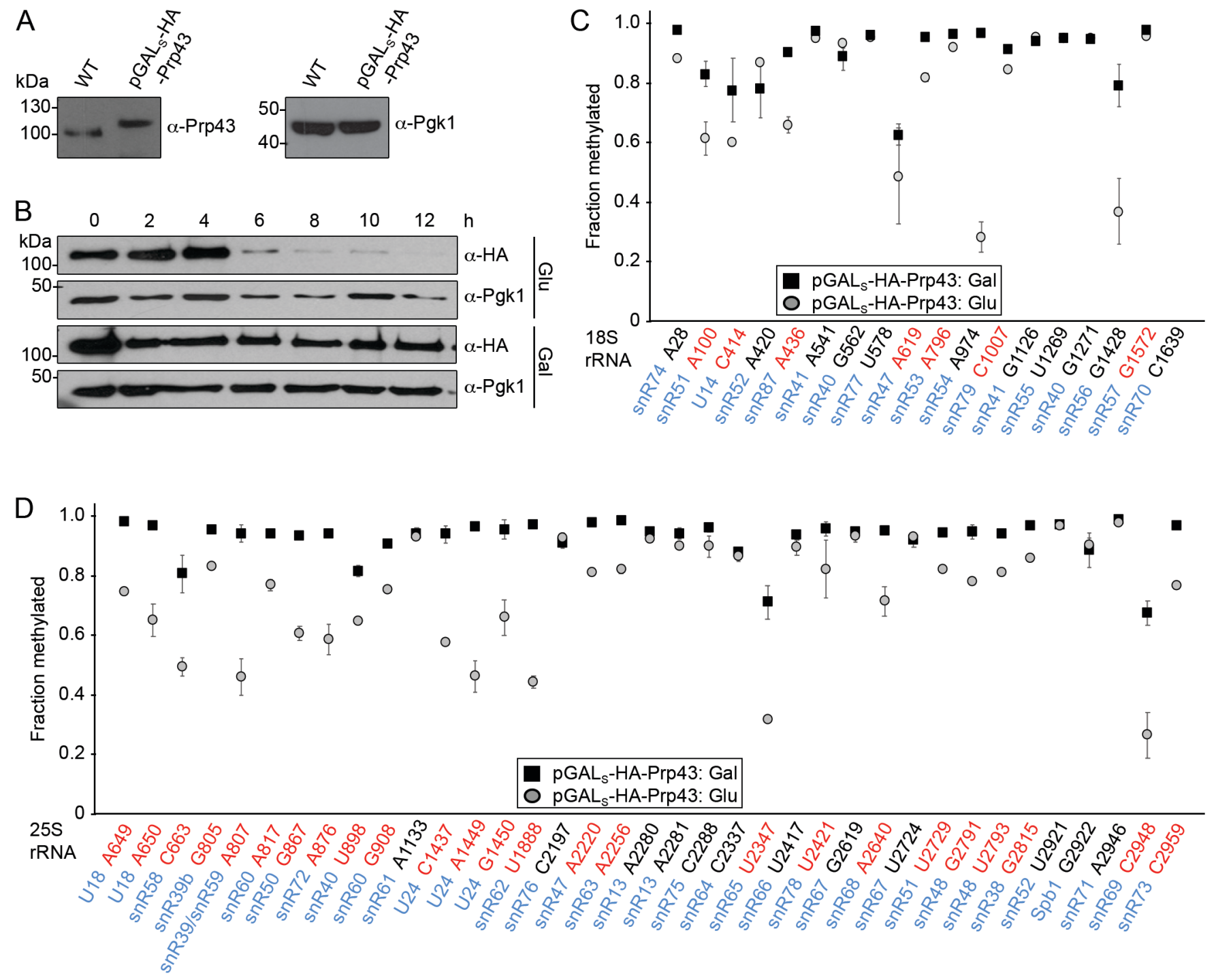

E
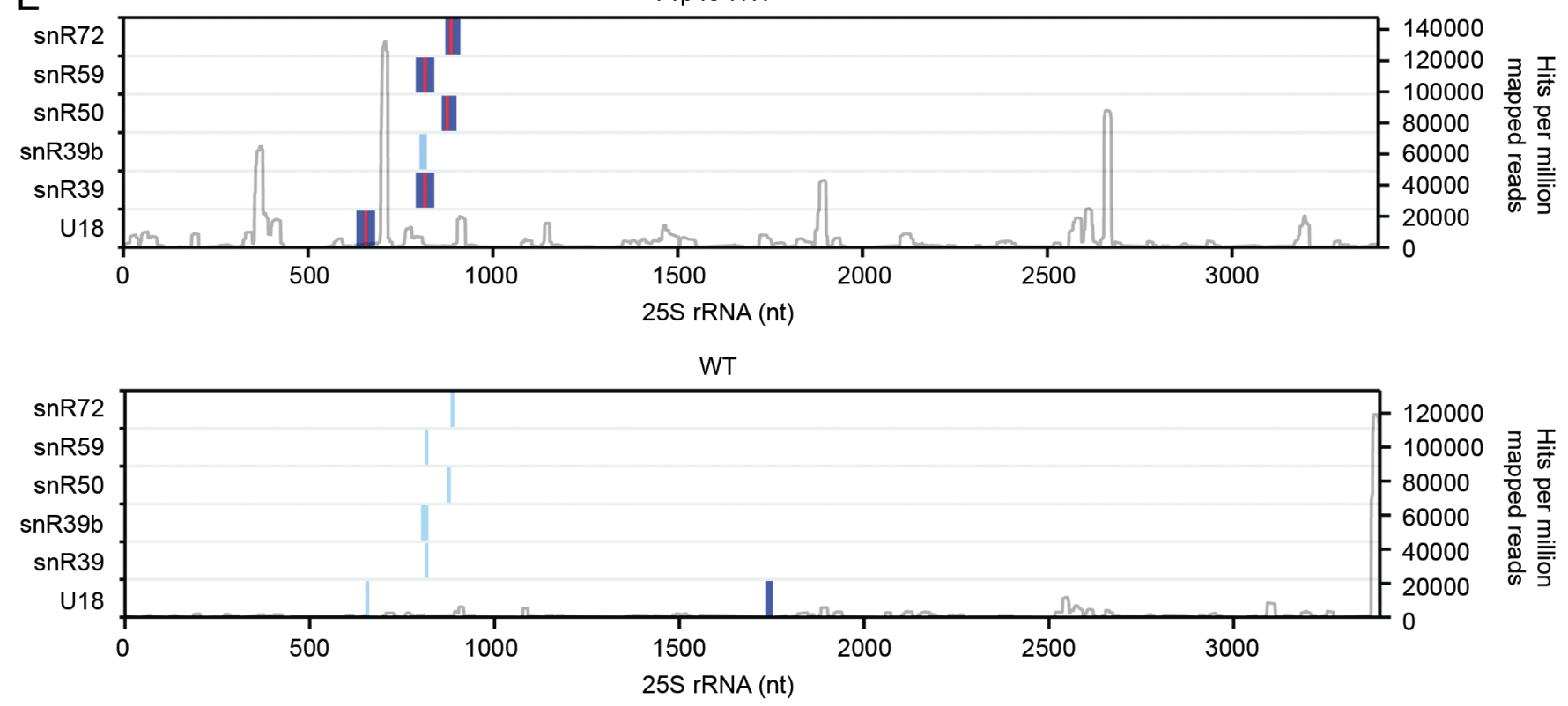

Aquino et al., Figure 8 


\section{SUPPLEMENTARY MATERIAL}

RNA helicase-mediated regulation of snoRNP dynamics on pre-ribosomes and rRNA 2'O-methylation

Gerald Ryan R. Aquino, Nicolai Krogh, Philipp Hackert, Roman Martin, Jimena Davila Gallesio, Robert W. van Nues, Claudia Schneider, Nicholas J. Watkins, Henrik Nielsen, Markus T. Bohnsack, Katherine E. Bohnsack

\section{SUPPLEMENTARY FIGURES}

Supplementary Figure S1. RNase H analysis of Am876, Um898, Um2724 and Gm2619 in snoRNA deletion strains. (A-D) RNA from wild type yeast or $4 s n r 78-72,4 s n r 40$ or $\Delta s n r 67-$ snr53 annealed to chimeric RNA-DNA oligonucleotides targeting the 25S-Am876, 25SUm898, 25S-Um2724 or 25S-Gm2619 sites as appropriate was either treated with RNase H $(\mathrm{RH},+)$ or left untreated (-). Samples were separated by denaturing agarose gel electrophoresis and northern blotting was performed using probes hybridising both upstream and downstream of the modification sites. Specific cleavage products are indicated by arrow heads; Asterix indicates non-specific cleavage products.

Supplementary Figure S2. The levels of snoRNAs guiding Dbp3-dependent 2'-Omethylation are not affected by the absence of Dbp3. (A) The numbers of sequencing reads mapping to each snoRNA was determined in the RMS datasets for wild type yeast and the $\Delta d b p 3$ strain. Relative snoRNA levels from two biologically independent RMS experiments in shown as mean \pm standard error. (B) RNA extracted from wild type yeast or cells of the $\triangle d b p 3$ strain was separated by denaturing PAGE and subjected to northern blotting using probes hybridising to the indicated snoRNAs and the 5S rRNA was used as a loading control.

Supplementary Figure S3. Structure and maturation of the U24 snoRNA. (A and B) RMS sequencing reads derived from wild type yeast and the $\Delta d b p 3$ strain were mapped to the annotated U24 sequence +/- $50 \mathrm{nt}$, and after normalisation for expression level, the numbers of reads mapping to each nucleotide were determined. Profiles for the 5' end (A) and 3' end (B) are shown. Nucleotides of box $C(B)$ and box $D(C)$ are underlined and the annotated 5 ' and 3' ends (58) are indicated. (C) Schematic view of the secondary structure of the pre-U24 snoRNA with key features indicated. Basepairing is shown according to (59).

Supplementary Figure S4. Levels of box H/ACA snoRNAs on pre-ribosomes in cells lacking Dbp3 compared to wild type. Wild type yeast and the $\Delta d b p 3$ strains were used to prepare cell extracts that were separated by sucrose density gradient centrifugation. Fractions containing either (pre-)ribosomal complexes or non-(pre-)ribosome associated proteins were 
pooled and RNA was extracted. Polyadenylation and reverse transcription were performed and the level of each of the 75 yeast snoRNAs in each sample was determined by qPCR. The relative distribution of each box H/ACA snoRNA between (pre-)ribosome-bound and nonribosome-associated fractions was calculated and differences in this ratio between the wild type and $\triangle d b p 3$ strains are shown graphically. Three independent experiments were performed and the data are presented as mean \pm standard deviation. \# indicates a snoRNA that is observed to accumulate on pre-ribosomes upon depletion of many other RNA helicases and ribosome AFs, and is therefore considered unspecific.

Supplementary Figure S5. Overview of rRNA 2'-O-methylations affected in $\Delta d b p 3$ mapped on the secondary structure of the 25S rRNA. 2'-O-methylations reduced in $\triangle d b p 3$ and the snoRNAs that guide them were highlighted on the secondary structure of the $25 \mathrm{~S}$ rRNA (41) in red and the pre-rRNA nucleotides involved in basepairing interactions with the guiding snoRNAs are indicated in green. Extra snoRNA basepairing is shown in blue. The areas shown in Figure 5 as magnified views are indicated with boxes with labels corresponding to the individual panels.

\section{Supplementary Figure S6. Pre-ribosome-association and overexpression of snR67. (A)} Whole cell extracts prepared from wild type yeast and the $\Delta d b p 3$ strain were separated by sucrose density gradient centrifugation. RNA from individual fractions was separated by denaturing PAGE and analysed by northern blotting using probes to detect the snR67 snoRNA. The fractions containing "free" snoRNAs and pre-ribosome-associated snoRNAs are indicated. The upper panel is reproduced from Figure 5F. (B) RNA extracted from wild type yeast or $\triangle d b p 3$ cells transformed with either an empty pRS416 plasmid or a construct for overexpression of snR67 (snR67oE) was separated by denaturing PAGE and subjected to northern blotting for the snR67 and snR64 snoRNAs. Mature 5.8S rRNA, visualised by methylene blue staining, served as a loading control.

Supplementary Figure S7. snoRNA levels in pGALs-HA-Prp43 grown in galactose- and glucose-containing media. The numbers of sequencing reads mapping to each snoRNA was determined in the RMS datasets for the pGALs-HA-Prp43 strain grown in glucose- and galactose-containing media. Relative snoRNA levels from two biologically independent RMS experiments in shown as mean \pm standard error. 


\section{SUPPLEMENTARY TABLES}

Supplementary Table 1. DNA oligonucleotides used in this study. Primers used for qPCR

analysis of snoRNA levels on pre-ribosomes are listed in [29].

\begin{tabular}{|c|c|c|}
\hline Name & equence $\left(5^{\prime}-3^{\prime}\right)$ & Application \\
\hline oMB1461 & SGGTTTTAATTGTCCTA & Northern blot probe (ITS1) \\
\hline oMB1468 & TGAGAAGGAAATGACGCT & Northern blot probe (ITS2) \\
\hline oMB2510 & ATATTAGGATCCGCCATGACAAAGGAAGAAATCGCAG & Molecular cloning pMB312 \\
\hline oMB2511 & ACGCGGTACCCTAATCGAAAGTAATTTTTTTTGGGTTTC & Molecular cloning pMB312 \\
\hline oMB2594 & $\begin{array}{l}\text { CAGTTTGATATATAGGGGATTAAACGGATCCCCGGGT } \\
\text { TAATTAA }\end{array}$ & Genomic deletion of $D B P 3$ \\
\hline oMB2595 & $\begin{array}{l}\text { AACCCCTAATCGAAAGTAATTGAATTCGAGCTCGTTTA } \\
\text { AAC }\end{array}$ & Genomic deletion of $D B P 3$ \\
\hline oMB2625 & TGATCAGTCGACCGTTATTTATTTT & 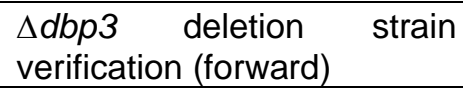 \\
\hline oMB2626 & CCATAGAGTTTAATCAGGCAAGAGA & $\begin{array}{lcc}\Delta d b p 3 & \text { deletion } & \text { strain } \\
\text { verification (reverse) } & \\
\end{array}$ \\
\hline oMB3405 & $\begin{array}{l}\text { GAAGATAGACGAAATAGGAACAACAAACAGCTTATAA } \\
\text { GCACCCAATAAGTGCGTTCGGATCCCCGGGTTAATTA } \\
\text { A }\end{array}$ & Genomic deletion of $R R P 6$ \\
\hline oMB3406 & $\begin{array}{l}\text { GAGGTCTTAAATGAAAATTACCATAATTTATAAATAAAA } \\
\text { AAATACGCTTGTTTTACATAAGAATTCGAGCTCGTTTA } \\
\text { AAC }\end{array}$ & Genomic deletion of $R R P 6$ \\
\hline oMB3409 & TGACAGAACCATTTCATGTTCAATA & $\begin{array}{lll}\text { srrp6 deletion } & \text { strain } \\
\text { verification (forward) } & \end{array}$ \\
\hline oMB3412 & ATGTGAAGAAAAGAATTCCTGACAC & $\begin{array}{lcc}\Delta r r p 6 & \text { deletion } & \text { strain } \\
\text { verification (reverse) } & \\
\end{array}$ \\
\hline oMB5759 & $\begin{array}{l}\text { CGATAGTATAACCTTATAAAC } \\
\text { GACTGCAAGAATAATAATGC }\end{array}$ & $\begin{array}{l}\text { Genomic integration of } \\
\text { pGALs promoter for } P R P 43\end{array}$ \\
\hline oMB5760 & $\begin{array}{l}\text { GGAATAGAGGTCTCAACTGGATCCGGGTGTTCGGAC } \\
\text { GAGAATCTTCTTTTGGAACCCATCGATGAATTCTCTGT } \\
\text { CG }\end{array}$ & $\begin{array}{l}\text { Genomic integration of } \\
\text { pGALs promoter for } P R P 43\end{array}$ \\
\hline oMB6716 & $\begin{array}{l}\text { ATATATGGATCCGGTTATACTGCGT } \\
\text { CTGG }\end{array}$ & Molecular cloning pMB1401 \\
\hline oMB6717 & $\begin{array}{l}\text { ATATATGCGGCCGCTGGTCCAATATTTGACGTTTCTA } \\
\text { G }\end{array}$ & Molecular cloning pMB1401 \\
\hline oMB6720 & $\begin{array}{l}\text { GTTAATTACTTAGTATTAGACCAGGCAGACAGAATGTT } \\
\text { GGAAAAAGG }\end{array}$ & $\begin{array}{l}\text { Site-directed mutagenesis } \\
\left.\text { (Dbp3 }{ }_{E 263 Q}\right)\end{array}$ \\
\hline oMB6721 & $\begin{array}{l}\text { CCTTTTTCCAACATTCTGTCTGCCTGGTCTAATACTAA } \\
\text { GTAATTAAC }\end{array}$ & $\begin{array}{l}\text { Site-directed mutagenesis } \\
\left.\text { (Dbp3 }{ }_{E 263 Q}\right)\end{array}$ \\
\hline oMB1465 & CTCCGCTTATTGATATGC & $\begin{array}{l}\text { Northern blot probe } 25 \mathrm{~S} \\
\text { rRNA (RNase H) }\end{array}$ \\
\hline oMB7263 & GCCTGCTATGGTTCAGCGACG & $\begin{array}{l}\text { Northern blot probe } 25 S \\
\text { rRNA (RNase H) }\end{array}$ \\
\hline oMB6327 & CTGTCTAGATGAACTAACACC & $\begin{array}{l}\text { Northern blot probe } 25 S \\
\text { rRNA (RNase H) }\end{array}$ \\
\hline oMB8611 & AAAACTCGAGCTTCAACCTGTACGTGGATGG & $\begin{array}{l}\text { Cloning snR67 } \\
\text { overexpression }\end{array}$ \\
\hline oMB8612 & AAAAGGTACCGAGGAGTGATAATGGCAATTAAGAC & $\begin{array}{l}\text { Cloning snR67 for } \\
\text { overexpression }\end{array}$ \\
\hline oMB1537 & GGTGATTAAACGACAGCATTGTCAAAGACTAGTCGA & Northern blot probe snR59 \\
\hline oMB1633 & CAGTGTTTGTTGTTTGTAAAATCAG & Northern blot probe snR67 \\
\hline oMB1635 & CAGTCATTTCAAAGATCCGCTTGG & Northern blot probe snR67 \\
\hline oMB6021 & GATTCAGAAACTCTAGTTTG & Northern blot probe snR78 \\
\hline oMB6022 & GAATAAACGTTCTAATCAC & Northern blot probe snR78 \\
\hline oMB6887 & GATGGTGATAAGTTACGACAGC & Northern blot probe snR39 \\
\hline
\end{tabular}




\begin{tabular}{|c|c|c|}
\hline oMB1544 & CGACAGCATCGTCAATGACTAGTCGAATATGTATTGG & Northern blot probe snR39 \\
\hline oMB1545 & GGTGATAAGTTACGACAGC & Northern blot probe snR39 \\
\hline oMB6772 & GGTATGTCTCATTCGGAACTCAAAG & Northern blot probe U24 \\
\hline oMB6773 & GTGATAATTGGTATGTCTCATTCGG & Northern blot probe U24 \\
\hline oMB1541 & GCTGCAAATTGCTACCTCTTTCA & Northern blot probe snR50 \\
\hline oMB1548 & TGCTAGTCACTTTTTGGAATGCC & Northern blot probe snR39b \\
\hline oMB6689 & CTGAGTACTTGTGGCATCCATG & Northern blot probe snR40 \\
\hline oMB1928 & TTCCAAAGGAATCATCG & Northern blot probe snR55 \\
\hline oMB1551 & CATTTGATGAGACGTTTTCTTCA & Northern blot probe snR72 \\
\hline oMB6770 & GGTTTATAGCATTGTCACTAAGGACG & Northern blot probe snR69 \\
\hline oMB6771 & GCTGGGTTTATAGCATTGTCACTAAG & Northern blot probe snR69 \\
\hline oMB1546 & GTCAGATACTGTGATAGTC & Northern blot probe U18 \\
\hline oMB1550 & CTTCATTTCGATAGTATGTTCAATCAG & Northern blot probe snR60 \\
\hline oMB2213 & GTCACAGGCGAAATATCATCAAAGTTAATC & Northern blot probe snR73 \\
\hline oMB6745 & CCCGCTAAAGCATTGTCACTC & Northern blot probe snR76 \\
\hline oMB6888 & CGTGCGTCTGATTATGGTCC & Northern blot probe snR63 \\
\hline oMB1828 & $\begin{array}{l}\text { TCCTTTAGAGATGATAAAGACAACTTACAAGTACAGTT } \\
\text { TTTTGTTGGTATCTCATCGGATCCCCGGGTTAATTAA }\end{array}$ & Genomic deletion of SNR67 \\
\hline \multirow[t]{3}{*}{ oMB1843 } & $\begin{array}{l}\text { AAATACATGTTCCTTGAGAAAACTTTTGATGTTTAATG } \\
\text { TGTAAATATCTCATTAAGAATTCGAGCTCGTTTAAAC }\end{array}$ & Genomic deletion of SNR67 \\
\hline & & $\begin{array}{l}\text { Genomic deletion of SNR72- } \\
78\end{array}$ \\
\hline & & $\begin{array}{l}\text { Genomic deletion of SNR72- } \\
78\end{array}$ \\
\hline oMB1832 & $\begin{array}{l}\text { TAGTACCTTAACACATGACGAAGATGAAAATTAAACAT } \\
\text { GAATTCAAGGAAAAATGCGGATCCCCGGGTTAATTAA }\end{array}$ & Genomic deletion of SNR40 \\
\hline oMB1848 & $\begin{array}{l}\text { TCATAATACAGTCACAGATGTGAGAGAAAAAAAAAAAA } \\
\text { GAAAATAAAGGAAAATGGAATTCGAGCTCGTTTAAAC }\end{array}$ & Genomic deletion of $S N R 40$ \\
\hline oMB7718 & GCCCTAACTTTCCACCTCGC & $\begin{array}{l}\Delta s n r 67 \text { deletion strain } \\
\text { verification }\end{array}$ \\
\hline oMB7719 & CTAATTCCAATACGAAGAGC & $\begin{array}{l}\Delta s n r 67 \text { deletion strain } \\
\text { verification }\end{array}$ \\
\hline oMB7720 & CAAATAGACAAGCATATGTC & $\begin{array}{l}\Delta s n r 72 \text { deletion } \\
\text { verification }\end{array}$ \\
\hline oMB7721 & CATCTAGTTCTTTGTCCAAAG & $\begin{array}{l}\Delta s n r 72 \text { deletion } \\
\text { verification }\end{array}$ \\
\hline oMB7714 & GAACACAACATTAAAAATGG & $\begin{array}{l}\Delta s n r 40 \text { deletion } \\
\text { verification }\end{array}$ \\
\hline oMB7715 & CTCCGAGCCATATCGGAAAG & $\begin{array}{l}\Delta s n r 40 \text { deletion strain } \\
\text { verification }\end{array}$ \\
\hline
\end{tabular}

\section{Supplementary Table 2. Plasmids used in this study}

\begin{tabular}{|l|l|l|}
\hline Name & Description & Application \\
\hline pMB031 & pRS415 & Protein expression in yeast \\
\hline pMB312 & A21-Dbp3 & Protein expression in E. coli \\
\hline pMB1400 & A21-Dbp3 2 263Q & Protein expression in E. coli \\
\hline pMB1401 & pRS415-Dbp3+/-500 & Yeast complementation \\
\hline pMB1402 & pRS415-Dbp3 E2630 +/-500 & Yeast complementation \\
\hline pMB1730 & pRS416-ACTintron-pGAL $1 ~$ & Yeast complementation \\
\hline pMB1732 & pRS416-ACTintron-pGAL 1 -SNR67 & Yeast complementation \\
\hline
\end{tabular}


Supplementary Table 3. Yeast strains used in this study

\begin{tabular}{|l|l|l|}
\hline Name & Geneotype & Reference \\
\hline YMB006/BY4741a & MATa;his 1;leu2A0;met1540;ura40 & Euroscarf \\
\hline YMB724 & YMB006; dbp3::kanMX6 & This study \\
\hline YMB909 & YMB006; rrp6::kanMX6 & This study \\
\hline YMB1088 & YMB006; pGALs prp43 natNT2 & This study \\
\hline YMB1487 & YMB006; pMB031 (LEU2) & This study \\
\hline YMB1538 & YMB724; pMB1401 (LEU2) & This study \\
\hline YMB1539 & YMB724; pMB1402 (LEU2) & This study \\
\hline YMB1540 & YMB724; pMB031 (LEU2) & This study \\
\hline YMB1566 & YMB006; nop1-HTP (URA3) & This study \\
\hline YMB1567 & YMB724; nop1-HTP (HIS3) & This study \\
\hline YMB1718 & YMB006; SNR78-SNR72::natNT2 & This study \\
\hline YMB1720 & YMB724; SNR78-SNR72::natNT2 & This study \\
\hline YMB1748 & YMB006; SNR40::natNT2 & This study \\
\hline YMB1750 & NOP65DAmP kanMX & Dharmacon \\
\hline YMB1752 & YMB1750; dbp3::kanMX6 & This study \\
\hline YMB1715 & YMB006; SNR67-SNR53::natNT2 & This study \\
\hline YMB1903 & YMB006; pMB1730 (URA3) & This study \\
\hline YMB1905 & YMB724; pMB1730 (URA3) & This study \\
\hline YMB1895 & YMB006; pMB1732 (URA3) & This study \\
\hline YMB1899 & YMB724; pMB1732 (URA3) & This study \\
\hline
\end{tabular}

Supplementary Table 4. Chimeric oligonucleotides used for RNase H-based cleavage

assays. $\mathrm{mN}$ indicates 2'-O-methylated RNA nucleotides and $\mathrm{dN}$ are DNA nucleotides.

\begin{tabular}{|l|l|}
\hline Name/Target modification & Sequence (5'-3') \\
\hline $25 S-A m 876$ & $m A m C m G d T d C d A d G m A m A m C m C m G m C m U m A m C m G m A m G$ \\
\hline $25 S-U m 898$ & $m$ mCmAdAdAdTdTmCmGmAmCmGmAmUmCmGmAmUmUmU \\
\hline $25 S-G m 2619$ & $m$ GmCmCdCdCdAdGmCmCmAmAmAmCmUmCmCmCmCmAmC \\
\hline $25 S-U m 2724$ & mUmGmAdAdAdAdTmCmAmAmAmAmUmCmAmAmGmGmGmG \\
\hline
\end{tabular}



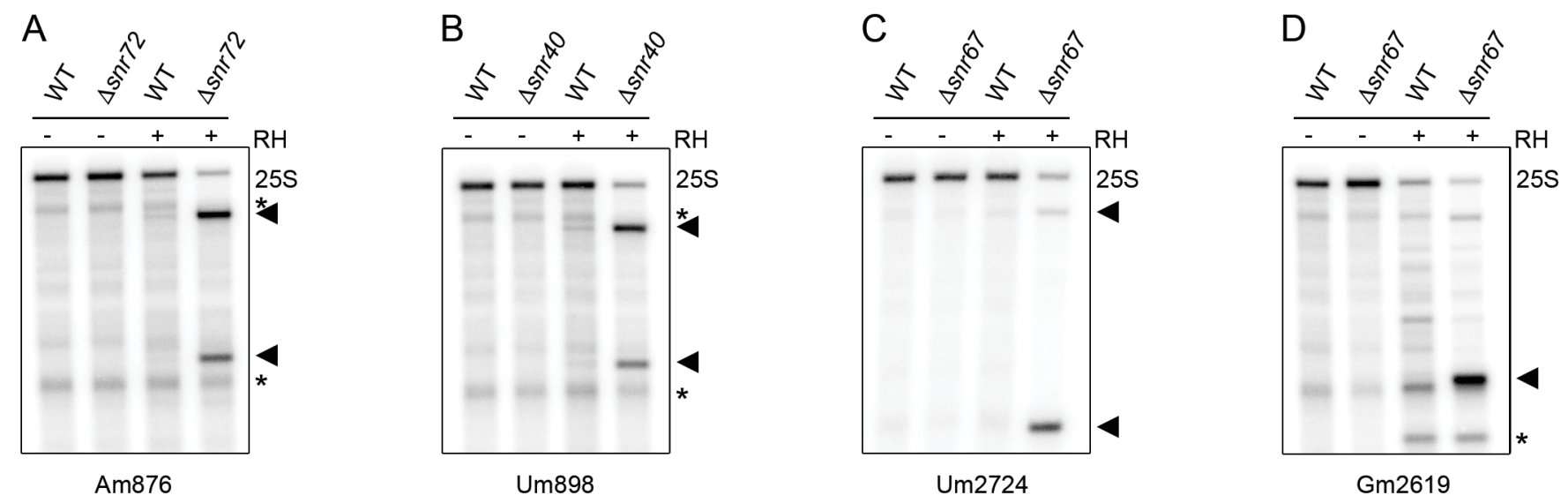

Aquino et al., Supplementary Figure S1 

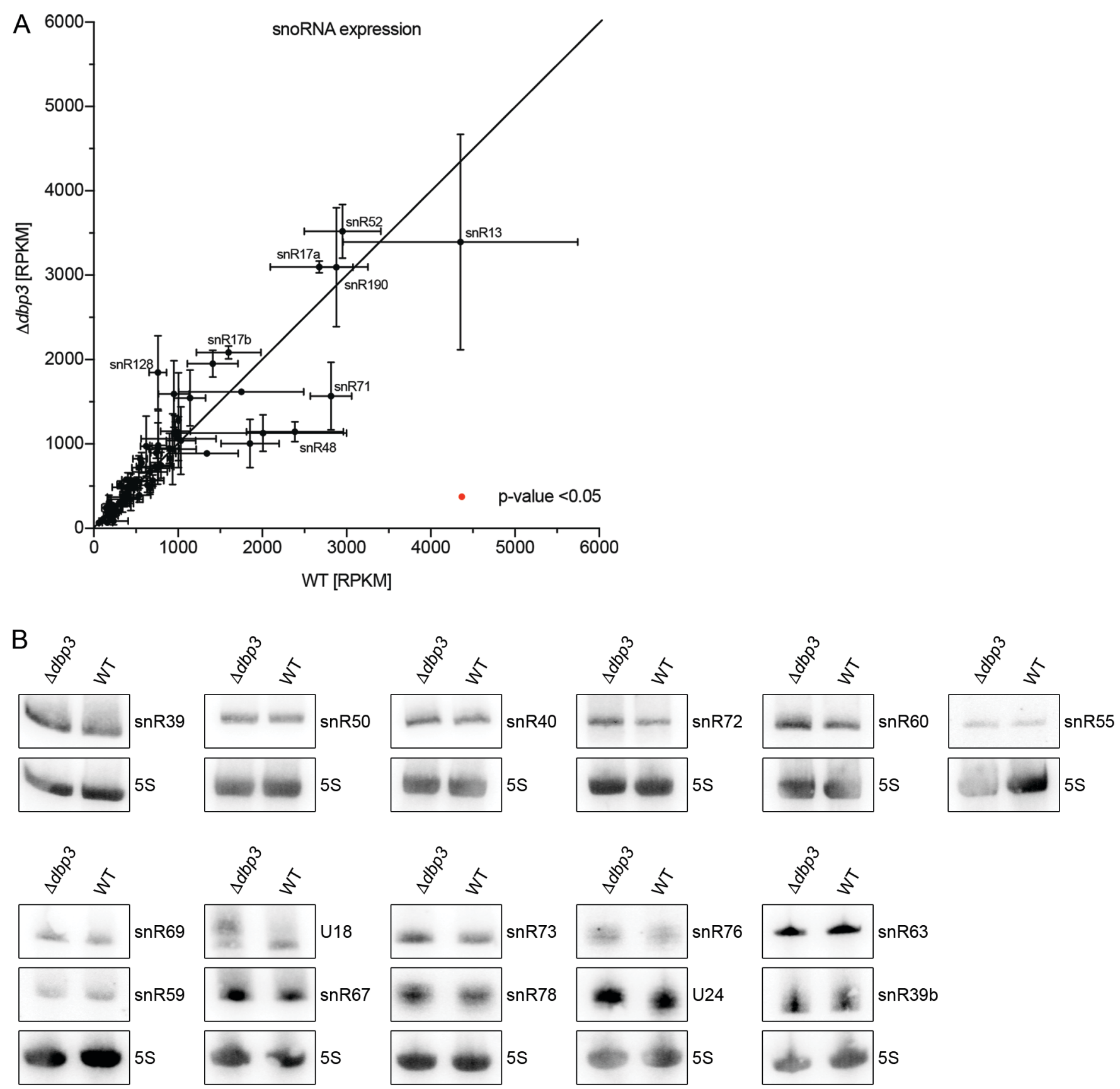

Aquino et al., Supplementary Figure S2 

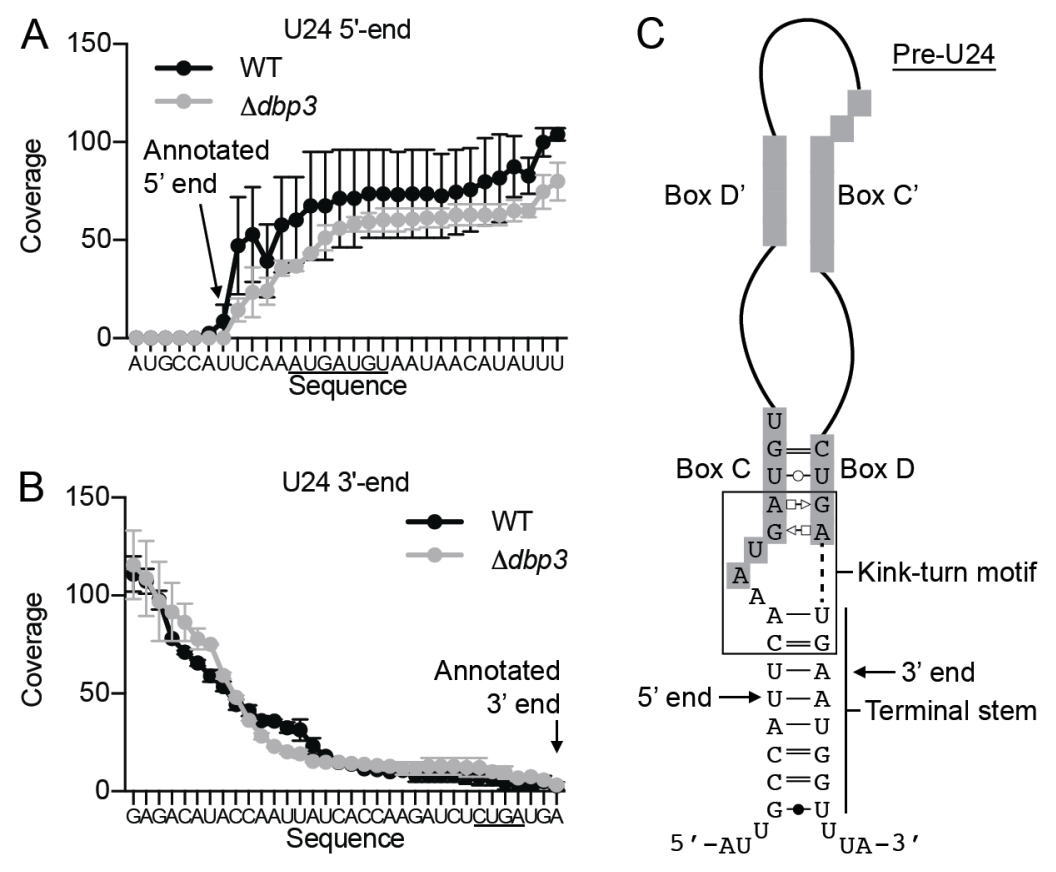

Aquino et al., Supplementary Figure S3 


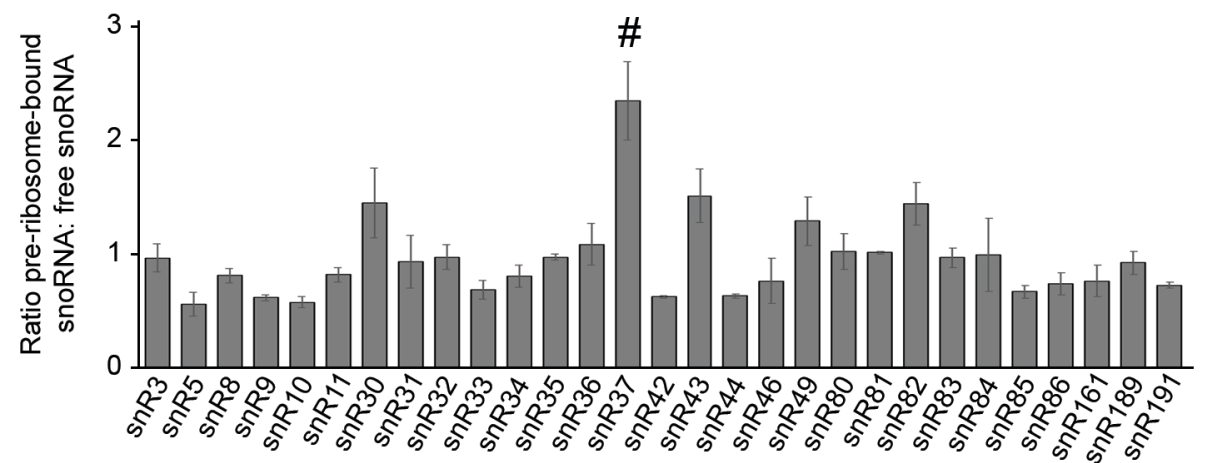

Aquino et al., Supplementary Figure S4 


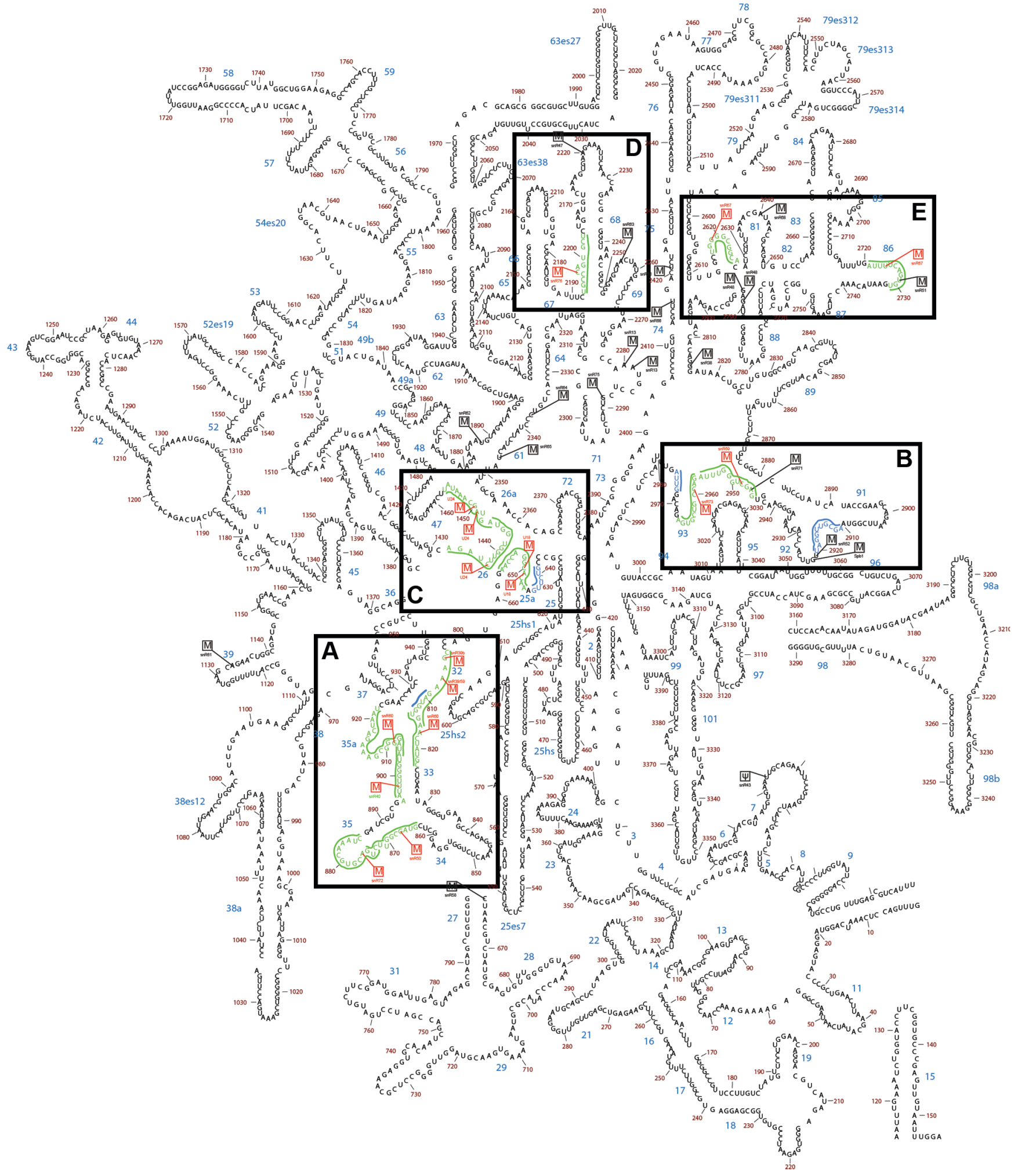

Aquino et al., Supplementary Figure S5 
A

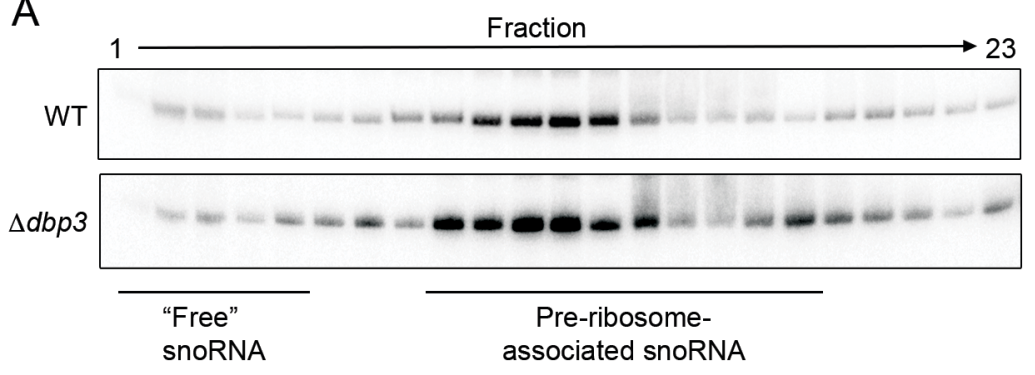

B

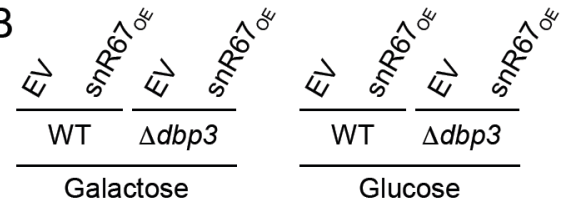

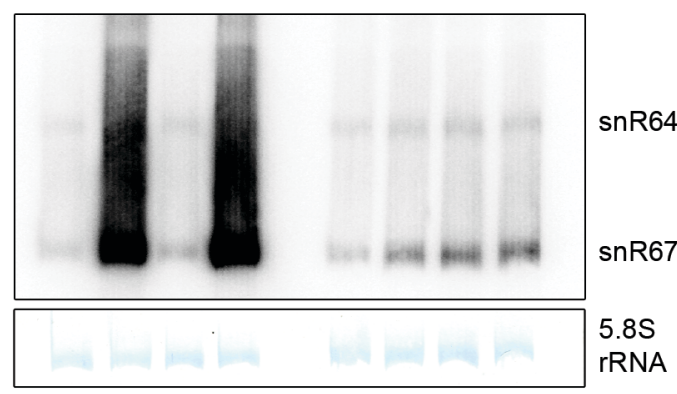

Aquino et al., Supplementary Figure S6 


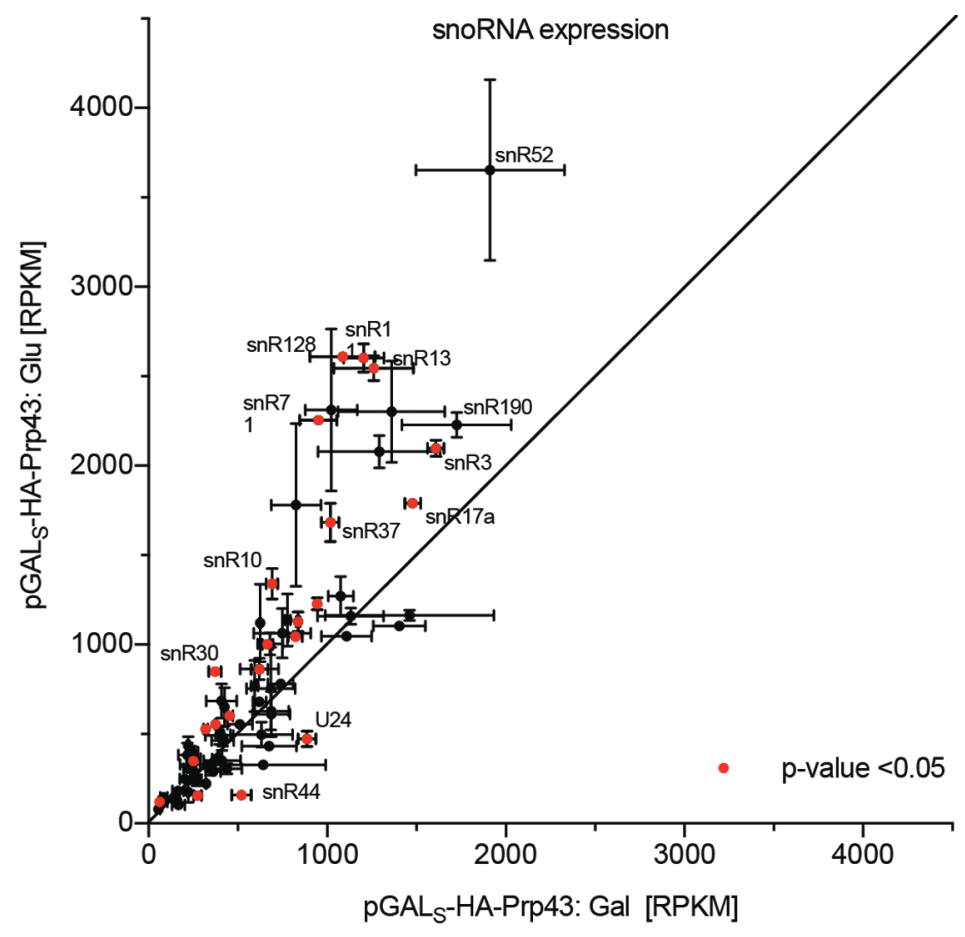

Aquino et al., Supplementary Figure S7 


\section{Chapter Five: Discussion}

The architecture and functionality of ribosomes are achieved by accurate assembly of its RNA and protein components. The assembly process includes modification, processing and proper folding of the pre-rRNAs, concurrent with the binding of r-proteins and remodeling of RNAprotein interactions along the maturation pathway. These RNA and RNP structural transitions can be driven by RNA helicases using the energy of NTP binding and hydrolysis. To date, functional insights have been obtained for RNA helicases Prp43, Has1, Sbp4, Mak5, Dbp4, Dbp5, Rok1, Kre33, and Dhr1 (Bohnsack et al., 2008, 2009; Brüning et al., 2018; Dembowski et al., 2013a; Gnanasundram et al., 2019; Khoshnevis et al., 2016; Liang \& Fournier, 2006; Martin et al., 2014; Neumann et al., 2016; Pertschy et al., 2009; Sardana et al., 2015; Sharma et al., 2015). In general, these RNA helicases play important roles in processes such as promoting the release of snoRNP complexes from pre-ribosomal particles, regulating prerRNA processing, facilitating incorporation of r-proteins, and association and release of AFs.

Using molecular and biochemical assays coupled with high-throughput methods, this work sheds light on the functions of the two DExD box RNA helicases Dbp3 and Dbp7. We showed that $\mathrm{Dbp} 3$ regulates snoRNP dynamics on pre-ribosomes and is involved in the 3' processing of the intron-encoded snoRNAs U18 and U24, and thereby promotes efficient 2'-O-methylation of numerous nucleotides within the 25S rRNA. In contrast, we propose that Dbp7 mediates early folding and stabilization of inter-domain interactions of the pre-rRNA by promoting exchange of the folding chaperones Npa1 complex and snR190 for the r-protein uL3. Together, the characterization of these two RNA helicases contributes to the knowledge on RNA helicase function during ribosome biogenesis.

\subsection{RNA helicases have distinct remodeling functions to drive LSU maturation events}

Thirteen RNA helicases (Dbp2, Dbp3, Dbp5, Dbp6, Dbp7, Dbp9, Dbp10, Drs1 Has1, Mak5, Prp43 and Sbp4) are implicated in LSU biogenesis (Martin et al., 2013). In-depth characterization of several of them have provided important insight into their functions in the LSU assembly process. For example, the multifunctional Prp43 has been suggested to release several snoRNAs that guide modifications at numerous nucleotides in $25 \mathrm{~S}$ rRNA sequence (Bohnsack et al., 2009). Most importantly, our CLASH data revealed direct binding of Prp43 to the basepaired snoRNA and pre-rRNA sequences involved and therefore supports a direct role of Prp43 in mediating the release of this subset of snoRNAs from pre-ribosomes. The failure to release the snoRNAs can affect the snoRNP dynamics on the pre-ribosomes and may result to inefficient modification of the pre-rRNAs. This was indeed observed from our RiboMeth-Seq (RMS) data showing sub-stoichiometric level of 2'-O-methylation of various 
nucleotides upon depletion of Prp43. While all these observations present a processive unwinding of snoRNA-pre-rRNA basepairing by Prp43, our findings also suggest that other RNA helicases using a different mechanism can promote the release of snoRNPs from preribosomal complexes. In particular, in the absence of Dbp3, we observed accumulation of several snoRNPs in pre-ribosomal particles. Additionally, we found numerous overlap in the Prp43-affected 2'-O-methylation sites when Dbp3 is lacking, suggesting that Dbp3 has a distinct but related function in promoting 2'-O-methylation. However, it remains likely that the effect on 2'-O-methylation as well as the accumulation of snoRNAs on pre-ribosomes are downstream effects of the actual remodeling activity of Dbp3. Given the typically nonprocessive unwinding nature of DExD RNA helicases and the effect on various 2'-Omethylated sites, we suspect that the remodeling activity of Dbp3 on a specific pre-rRNA region induces a long-range rearrangement that indirectly affects snoRNPs dynamics in the preribosome. Unfortunately, we were not able to identify the pre-rRNA binding site(s) of Dbp3 using the CRAC approach. This might be attributed to the nature of Dbp3 interaction with the pre-ribosome, i.e. transient binding or incompatibility of pre-RNA nucleotides and amino acids for facilitating the contact points to crosslinking. Structural insights into pre-ribosomal particles containing or lacking Dbp3 will likely be necessary to fully understand the molecular function of Dbp3.

Recently, functional characterizations have also been done to DExD RNA helicases Has1, Mak5, and Spb4 (Brüning et al., 2018). In addition to its role in SSU biogenesis, Has1 has been suggested to promote the stable incorporation of several r-proteins including uL24, uL29, eL37 and the release of specific AFs (Dembowski et al., 2013a). In the case of Mak5, its remodeling activity is proposed to be important for the recruitment of uL16. Sbp4, in contrast, is implicated in facilitating the association of export adaptor Arx1. A key basis for underlying the functional analyses of these RNA helicases was the identification of their RNA binding site(s) using CRAC. In this work, we have identified the pre-rRNA binding site of Dbp7 that corroborates well with its proposed remodeling function in promoting the release of Npa1 complex and snR190 to allow the incorporation of uL3. Comparison of the identified crosslinking sites of these fours RNA helicases strongly suggests unique protein binding sites reflecting their distinct remodeling functions. Additionally, analysis of protein composition of pre-60S particles isolated using each RNA helicase as a bait revealed components that support the proposed function of each RNA helicase. For example, the identification of uL3 in Dbp7-containing particle provides compelling evidence for stable incorporation of uL3 in the pre-ribosome promoted by the presence of Dbp7. Moreover, the identification of Npa1 complex, Dbp6 and Dbp9 in this particle supports the reported functional link and genetic interactions of these proteins in LSU biogenesis (Daugeron \& Linder, 1998; de la Cruz et al., 
2004; Dez et al., 2004; Rosado et al., 2007a). Aside from having distinct functions, RNA helicases also act at specific time point in the assembly process. This can also be inferred from the analysis of protein composition and in addition, from the pre-rRNAs present in the pre-60S particles isolated using each RNA helicase as bait. For instance, the identification mostly of 27SA pre-rRNA in Dbp7-contaning particle suggests a role in the early stages of LSU biogenesis, whereas the identification of 27SB pre-rRNA in Spb4-containing particle reflects a later role in the assembly, i.e. construction of export-competent pre-ribosome (Brüning et al., 2018). Interestingly, the identification of Mak5, Prp43, Dbp3, Dbp6, Dbp9 and Has1 in Dbp7contaning particle indicates their presence in the same pre-ribosomal particle and suggests that all these helicases likely acting at the same stage of assembly. Altogether, these observations point to the fact that LSU RNA helicases remodel different regions of preribosomes simultaneously or at different time points to drive diverse maturation events. How the individual functions of these various RNA helicases are synchronized and how these are also coordinated with other AFs remain to be outstanding questions.

\subsection{DExD-box RNA helicases dominate ribosome biogenesis}

Ribosome biogenesis is the cellular pathway that involves the largest number of RNA helicases and the majority (17 out of 21) of them are DExD box RNA helicases. This observation raises the question of why DExD box RNA helicases are preferred in this macromolecular machine assembly. The answer may largely lie in their specific biochemical properties and activities coupled with the complex nature of ribosome biogenesis.

DExD Box RNA helicases, like DNA helicases and other RNA helicases, use the NTPase cycle to regulate their affinity to nucleic acid sequences (Jarmoskaite \& Russell, 2011). However, the characteristics of their unwinding differ from those of other helicases in many intrinsic ways. First, this subfamily is mostly immobile when they do unwinding. This is in contrast to the DEAH box RNA helicases, which translocate along one of the RNA strands displacing the other complementary strand. This inability to translocate classifies DExD RNA helicases as nonprocessive and consequently, their unwinding capability is limited for duplexes longer than 1015 bp (Jarmoskaite \& Russell, 2011; Rogers et al., 1999). Although this may leave an impression that they are inefficient RNA helicases, helices found in structured RNAs rarely exceed 10 bp (Bellaousov et al., 2018). Hence, DExD box helicases can typically remodel these substrates without the need for high processivity and energy expenditure. To give an example, Dbp3 and Rok1 can unwind RNA duplexes of 10 bp in vitro (Garcia et al., 2012). Second, as they can unwind internally within RNA duplexes, DExD box RNA helicases do not need polarity of the RNA substrate (Yang et al., 2007). This is in contrast to DEAH RNA helicases, which mostly translocate from $3^{\prime}$ to 5 ' direction and requires single-stranded 
overhangs (Jarmoskaite \& Russell, 2014). Such single-stranded overhangs would likely be hardly available and/or accessible as the pre-rRNA undergo compaction very early on with the association of r-proteins and AFs (Gamalinda et al., 2014; Klinge \& Woolford, 2019; Konikkat \& Woolford, 2017). Hence, a localized remodeling within an already layered RNP that allows global structural transitions is ideal for ribosome assembly and maturation. This localized but efficient remodeling is a fitting role for DExD box RNA helicases. Our findings likely provide an example of this with the local remodeling activity of Dbp3 being transmitted to a large-scale structural rearrangement to modulate the dynamics of numerous snoRNPs in the preribosome. Notably, there are examples of non-DExD box RNA helicases implicated in ribosome biogenesis, e.g. in snoRNAs release. As mentioned earlier is the Prp43 implicated in the direct unwinding of several snoRNA-pre-rRNA interactions. This highlights the likewise need for processive unwinding. Another DEAH box helicase Dhr1 is proposed to promote the displacement of U3 from SSU pre-ribosomal particle, however, in a manner similar to DExD box RNA helicases (Sardana et al., 2015). Interestingly, few DExD RNA helicases likely directly unwind the basepairing of single snoRNAs with pre-rRNAs, e.g. Has1 and U14, Rok1 and snR30 (Brüning et al., 2018; Martin et al., 2014). This demonstrates specificity and variety in the action of DExD box RNA helicases, characteristics that further makes them ideal remodelers to drive the complex process of ribosome biogenesis. Furthermore, these observations suggest that a combination of processive and non-processive mechanisms provided by several RNA helicases is necessary to efficiently release snoRNAs from preribosomal particles.

\section{3 'Essentiality' of RNA helicases implicated in ribosome synthesis}

Dbp3 and Dbp7 are two of the three non-essential RNA helicases involved in ribosome biogenesis. The term 'non-essential' is defined as the ability of cells to remain viable in the absence of the referred protein. This is evident for Dbp3 as growth of a $\Delta d b p 3$ strain is comparable with the wild-type strain. In the case of a $\Delta d b p 7$ strain, a pronounced growth retardation is observed, with the strain showing twice the doubling time of a wild-type strain. Furthermore, deletion of the DBP7 OFR from the genome has been proven to be difficult. The difference in the growth phenotype of these two deletion strains can likely be attributed to the nature of their function. Dbp3 appears to function in the fine-tuning of rRNA modification, and it has been shown that a sub-stoichiometric level of modification and the lack of some specific 2'-O-methylations is not especially detrimental to cells (Ojha et al., 2020; Sharma \& Lafontaine, 2015; Sloan et al., 2017). In humans, rRNA modifications are much abundant and many snoRNAs have overlapping basepairing sites. While our data suggest that modification of proximal sites occurs stochastically in yeast, an extent of hierarchical rRNA modification of proximal sites in humans may be necessary. This may prevent inefficient installation of modifications that is more than can be tolerated by cells. Indeed, higher eukaryotes have 
partitioned some of these modifications in later step of assembly with a dedicated RNA helicase to regulate snoRNP recruitment. An example is the DExD box RNA helicase DDX21 shown to facilitate the recruitment of SNORD56 and SNOR68, which both guide late-step modifications (Sloan et al., 2015).

In contrast, the function of Dbp7 in the recruitment of a crucial r-protein uL3 likely explains the very sick phenotype of $\Delta d b p 7$ strains. uL3 contacts the 5' and 3' end of the 25S rRNA in mature ribosomes (Ben-Shem et al., 2011). The early stabilization of the interaction between the 5 ' end and 3' end of the 25S is conserved across prokaryotes and eukaryotes, and therefore is a very important step in LSU assembly. This is further supported by the evident degradation of pre-ribosomal particle when Dbp7 is lacking. However, the cells remain viable in the absence of Dbp7. A possible reason for this could be a partial overlap in the function of Dbp7 with Dbp6 and Dbp9 as suggested by their reported genetic interaction (Daugeron \& Linder, 1998; de la Cruz et al., 2004; Dez et al., 2004; Rosado et al., 2007a). These three RNA helicases might have cooperative functions related to the Npa1 complex chaperone activity and the role of Dbp7 may not be as crucial as those of Dbp6 and Dbp9. The precise functions of Dbp6 and Dbp9 and the interplay among these RNA helicase remains, however, to be elucidated. Nevertheless, the aberrant growth phenotype and significant pre-ribosomal degradation when Dbp7 is lacking mirrors the phenotype of cells when one of the other LSU RNA helicases are conditionally depleted (Brüning et al., 2018; Burger et al., 2000; Daugeron et al., 2001; de la Cruz et al., 2004). Moreover, growth retardation is even worse when a catalytically inactive version of an essential RNA helicases is present in the cell (Brüning et al., 2018). This observation suggests that failure to release RNA helicase likely blocks the assembly process and hence, can be more detrimental than not having the RNA helicase at all.

\subsection{Towards understanding of RNA helicase functions in ribosome biogenesis}

\subsubsection{Direct and indirect functions of RNA helicases}

Ribosome biogenesis is a very intricate process that involves multiple events occurring in a parallel and stepwise manner. Consequently, this brings difficulty in designing approaches and developing techniques to determine the precise molecular function of AFs. Most of the readouts of genetic manipulations and current biochemical and molecular assays are rather downstream effects of their actual molecular function. Hence, a challenge lies in identifying 'consequences' versus 'direct function' and in narrowing down from an observed effect to the actual function. 
A good example of a molecular characterization that yielded observable indirect effects of the actual molecular function is exemplified by Dbp3. Initial characterization of this protein suggests its role in promoting pre-rRNA processing at the A3 site based on a pre-rRNA processing phenotype when Dbp3 is lacking (Weaver et al., 1997). Using RiboMeth seq, we observed many sites in the 25S rRNA having sub-stoichiometric 2'-O-methylation of $\Delta d b p 3$ strain. Additionally, northern blot and qPCR analyses of snoRNAs guiding these affected sites showed accumulation of several snoRNAs in the pre-ribosomal complexes in the absence of Dbp3. All these observations highlight consequences of lack of Dbp3 remodeling activity. Nevertheless, these findings suggest that the direct function of Dbp3 is possibly linked to prerRNA processing and 2'-O-methylation, and snoRNAs release from the pre-ribosome. In addition, we also uncover a putative function of Dbp3 in facilitating correct processing of the 3' ends of the intron-encoded snoRNAs U18 and U24. Interestingly, a lysine-lysine-X triplet ( $[K K X] ; X$ can be either serine, histidine, aspartate, glutamate, or glycine) is present at the $\mathrm{N}$ terminal of Dbp3. This sequence motif is also present in Nop56, Nop58 and Cbf5 (Guglielmi \& Werner, 2002), and hence supporting the involvement of Dbp3 in snoRNA processing. Our preliminary result from RNA immunoprecipitation (RNA-IP) experiment following crosslinking did not support the direct association of U18 and U24. We speculate that Dbp3 act as an adaptor to facilitate the recruitment of the exonuclease Rrp6. RNA-IP using Rrp6-TAP in a wild-type and $\triangle d b p 3$ background followed by northern blot detection of U18 and U24 can be done to support this model.

A direct function can be assigned to an RNA helicase when the RNA duplex it physically remodels has been identified. For example, the identification of snoRNAs-pre-rRNA duplex contacted by an RNA helicase provides compelling evidence for a direct function in the release of snoRNA from pre-ribosomes. Few RNA helicases have been reported to have a clear direct function in snoRNAs release. Two reported examples are the direct release of snR30 by LSU RNA helicase Rok1 (Martin et al., 2014) and of U14 by Has1 (Brüning et al., 2018). Dbp4 has also been implicated in the release U14 (Kos \& Tollervey, 2005; Soltanieh et al., 2015); however, Dbp4-containing pre-ribosomal particles was found to be lacking U14 (Soltanieh et al., 2015). This suggests that U14 is not directly bound by Dbp4 and therefore direct release of $U 14$ by Dbp4 is highly unlikely. In this work, we expand this list with addition of the multifunctional DEAH box protein Prp43. Our CLASH analysis of previously published CRAC data (Bohnsack et al., 2009) showed direct binding of Prp43 to the basepaired snoRNA and pre-rRNA sequences supporting its role in the direct release of snoRNP complexes from preribosomal particles. 
The roles of RNA helicases in the recruitment of $r$-protein and in the association and dissociation of AFs have also been reported including this work (Brüning et al., 2018; Dembowski et al., 2013a; Neumann et al., 2016). The recruitment of r-protein uL16 and exportadaptor Arx1 to pre-ribosomal particles are downstream the predicted timing of action of Mak5 and Spb4 respectively based on associated pre-rRNAs and protein composition. In the case of Dbp7, its co-existence with r-protein uL3 in the same pre-60S particle provide compelling support for a more direct role of Dbp7 in the recruitment of uL3. The current tools available to provide more conclusive evidence are rather limited. Furthermore, the complexity of ribosome assembly adds another layer of difficulty. For instance, the binding of ribosomal proteins might involve multiple weak binding events in the beginning before stable incorporation is achieved. Additional molecular snapshots from structural investigations are therefore indispensable in identifying direct functions of RNA helicases and in visualizing the molecular events underlying ribosome assembly.

\subsubsection{Targeting of RNA helicases to their RNA substrates}

CRAC experiments revealed that several DExD-box RNA helicases, i.e. Has, Sbp4, Dbp7, have more than one pre-rRNA contact site (Brüning et al., 2018). This raises the question whether it indicates multiple, exclusive binding events or multiple contact points arising from a single protein binding event. Mapping of crosslinking sites onto the pre-60S particles containing Has1 (Kater et al., 2017; Sanghai et al., 2018) showed close spatial proximity of the pre-rRNA binding sites. This is even more evident in the case of the three distinct crosslinking sites identified for Dbp7. Hence, several cross-linking sites can very likely indicate multiple contact points of an RNA helicase with the pre-rRNA to facilitate a single protein binding event. In contrast, the two pre-rRNA cross-linking sites identified for Sbp4 suggests two spatially distinct binding sites (Brüning et al., 2018). It is suggested that one crosslinking site represents a binding platform for the C-terminal tail of the helicase, which is predicted to bind RNA, whereas the other site potentially corresponds to the remodeling substrate of the helicase core. This speculation is consistent with the findings of Choudhury et al. (2020) showing that the human homologue of Spb4 (DDX55) has decreased affinity to double-stranded RNA in vitro when the $\mathrm{C}$-terminal is lacking. Furthermore, the absence of the $\mathrm{C}$-terminal region resulted in inability to associate with pre-ribosomes as well as non-specific binding of the helicase core to single-stranded RNA substrates. Aside from C-terminal domains, other ancillary domains can also function to target the RNA helicases to their RNA substrates. For example, the N-terminal extension called $Q$ domain in DExD box RNA helicases can mediate RNA binding substrate in addition to regulating ATP binding and hydrolysis (Bleichert \& Baserga, 2007). In the case of Dbp7 and Dbp3, the are no apparent ancillary domains adjacent the helicase core (Daugeron \& Linder, 1998; Weaver et al., 1997). 
Ancillary domains also exist in organellar and prokaryotic RNA helicases. C-terminal extensions are present in mitochondrial DExD box helicases including CYT-19 and Mss116 (Grohman et al., 2007; Mohr et al., 2008). In the case of Mss116, the basic, flexible C-terminal tail, similar to what is proposed for Spb4, has been shown to tether the RNA helicase to its substrate, thereby allowing remodeling of nearby RNA duplexes through the active site (Mallam et al., 2011, 2012). In bacteria, the DExD box protein DbpA has C-terminal domain that folds to adopt a RNA recognition motif (RRM) to bind specific RNA hairpins (Karginov et al., 2005; Tsu et al., 2001; Wang et al., 2006). Altogether, these observations imply that RNA helicases can be targeted to their target RNA or RNP complexes via a variety of different mechanisms. Furthermore, a single binding event may consist of two molecular interactions provided by different contact points: (1) docking of the RNA helicase onto its binding platform(s) facilitated by ancillary domains and (2) remodeling of its target RNA substrate in the active site or contact sites of the helicase core that are not within the active site.

\subsubsection{Structural and catalytic function of RNA helicases}

Investigations employing yeast complementation system revealed the necessity of the catalytic activity in the role of Dbp7 and Dbp3 in LSU biogenesis. This has also been observed in earlier functional characterizations of other RNA helicases involved in ribosome biogenesis (Brüning et al., 2018; Choudhury et al., 2019; Gallesio et al., 2020; Sharma et al., 2017). However, RNA helicase function independent of the catalytic activity remains possible. Moreover, RNA helicases may also have a dual function provided by a non-catalytic structural function and an energy-driven catalytic activity. This is reminiscent of the reported function of methyltransferase EMG1 in the recruitment of r-protein eS19 into pre-40S without the need for its enzymatic activity (Meyer et al., 2011). This implies that its presence on the particle per se provides a scaffolding and/or recruitment function. Functions in ribosome biogenesis independent of the catalytic activity has also been described for other modification enzymes including Dim1 and Bud23 and their human homologues (Haag et al., 2015; Lafontaine et al., 1995; White et al., 2008; Zorbas et al., 2015). This can also be anticipated for other catalytic proteins, including RNA helicases, and would provide further evidence to their non-unwinding function, e.g. molecular clamps to establish of RNA-protein and protein-protein interactions in RNP complexes. A concrete example is the DExD box protein Dbp5 (DDHX19 in humans), which is involved in the export of pre-ribosomal particles through its interaction with Mex67 but without the need of its ATPase cycle (Neumann et al., 2016). In the case of Dbp3 function in the context of snoRNA processing, it might be possible that Dbp3 acts as an adaptor for the binding of another protein as pre-requisite for the recruitment of the Rrp6 exonuclease that mediates the processing. Noteworthy, a sophisticated dual function is exemplified by Kre33, an acetyltransferase with an N-terminal DEAD-box like helicase domain (Sharma et al., 2015). Aside from its remodelling activity that promotes processing of SSU pre-rRNA, this enzyme 
presents a novel mechanism of rRNA acetylation using box C/D snoRNAs (Sharma et al., 2017). Its unwinding function promotes the binding of its associated guide snoRNAs snR4 and snR45, demonstrating that its remodeling activity is directly coupled to the modification function.

The ability to endow structural and catalytic functions, the observation of several pre-rRNA contacts for one single binding event, and the growing evidence indicating the need to stimulate ATPase activity for efficient unwinding, suggest that DExD RNA helicases may have different modes of action on their remodeling substrate. One speculative model could be that DExD box proteins bind to RNAs or RNPs and then alternately switch between "on" and "off" states until the target RNA sequence is remodeled. The "on" state represents ATP hydrolysis and release of the substrate while the "off" state represents the time before ATP hydrolysis. Several rounds of ATP hydrolysis, which reflects multiple binding events, might be needed until productive unwinding is achieved. Multiple binding events to unwind an RNA duplex has been proposed for the Ded1, a DEAD box RNA helicases implicated in translation initiation (Gupta et al., 2018; Raj et al., 2019). Another possible model could be that RNA helicases bind and remain in an "off" state, during which time they may fulfill a structural function or just merely tethered via their ancillary domain, and only later turn "on" when proper molecular orientation with the target substrate and subsequent unwinding is achieved. This model could partially explain why the binding site(s) of number of RNA helicases can be successfully "captured" using CRAC approach. With this model, it is intuitive that the ATPase activity of RNA helicases has to be regulated. Notably, it has been observed in vitro that the ATPase activity of DDX55 is significantly higher in the presence of double-stranded RNA (dsRNA) compared to singlestranded RNA (ssRNA) as substrate. In the presence of ssRNA, a minimal ATPase activity higher than background, i.e. in the absence of protein, is observed. Furthermore, the absence of C-terminal tail abolishes this minimal ATPase activity. These findings suggest that RNA helicases can have modest ATPase activity upon interacting with ssRNA and that C-terminal tail can positively influence this catalytic activity. Structural data to gain further insights into the mechanism of ATP hydrolysis in DExD box RNA helicases upon RNA substrate binding remains limited with only the crystal structure of Mss116 having double stranded RNA in its binding pocket (Mallam et al., 2011, 2012). Nevertheless, there is increasing knowledge on different mechanisms of how the catalytic activity of RNA helicases can be regulated (section 5.5). 


\subsubsection{Visualization of RNA helicases in pre-ribosomes and identification of RNA sequences} contacted by the helicase active site

Structural insights into pre-ribosomes containing RNA helicases are key in obtaining mechanistic understanding of RNA helicase functions in ribosome biogenesis. To date, there is a wealth of structural information from cryo-EM and X-ray crystallography investigations of pre-60S ribosomal particles (Barrio-Garcia et al., 2016; Greber, 2016; Kargas et al., 2019; Kater et al., 2017; Klingauf-Nerurkar et al., 2020; C. Ma et al., 2017; Malyutin et al., 2017; Sanghai et al., 2018; Wu et al., 2016; D. Zhou et al., 2019; Y. Zhou et al., 2019). So far, only structures of later, more compact pre-ribosomal particle have been resolved. Moreover, densities corresponding to RNA helicases in the available structures of early pre-60S particles are absent except for Has1 and partially for Spb4 (Kater et al., 2017; Sanghai et al., 2018; D. Zhou et al., 2019). This could imply that the nature of interaction of most RNA helicases with pre-ribosomal particles is very transient and dynamic. Additionally, it is very likely that these RNA helicases-containing early pre-60S particles are unstable and very heterogeneous, and therefore, are difficult to purify and analyze for structural investigations. Given the recent advances towards better resolution (Kuba et al., 2020; Schur, 2019; Zhang, 2019), cryoelectron tomography (cryo-ET) could potentially be utilized to visualize the positions of RNA helicases in pre-ribosomes and gain structural insights of this dynamic process in a native environment. Recently, direct visualization of ERAD machinery has been successfully done in Chlamydomonas reinhardtii using cryo-ET (Albert et al., 2020).

Careful examination of the density map assigned to Has1, for example in PDB 6COF (Kater et al., 2017), revealed that the RNA helicase is not directly contacting its identified RNA binding sites using CRAC (Brüning et al., 2018). Hence, even with the available structural information our mechanistic understanding of RNA helicase remodeling activity in pre-ribosomal particles remains limited. Structural investigations of particles purified using the RNA helicase of interest as bait could provide more useful information. Such an approach has been successfully done using the GTPase Lsg1 and yielded important insights into the key players and events in later stages of pre-60 biogenesis (Kargas et al., 2019). As ATP hydrolysis is coupled to substrate release for DExD box RNA helicase, the use of catalytically inactive versions of RNA helicase not able to hydrolyze ATP could enhance the purification process particularly for transiently interacting RNA helicases. Additionally, discerning the RNA sequence embedded in the active site is also crucial in our understanding of RNA helicases function particularly for those with multiple binding sites. Mass spectrometry-based identification of UV-crosslinked RNA-protein complexes can be a useful approach to determine which specific RNA sequences or contact sites are embedded in the active site of the RNA helicase (Kramer et al., 2014; Qamar et al., 2015; Stützer et al., 2016). Additionally, another variation of mass spectrometry called XL-MS 
(Linden et al., 2020; Parfentev et al., 2020; Yu \& Huang, 2018) can also be used to identify protein-protein interactions to explore the function of an RNA helicase as adaptor protein and/or as nucleation site to initiate the formation of a larger RNP. Moreover, this approach can reveal specific molecular interactions between RNA helicases and protein components of the RNPs, which in turn can provide further insights into the possible mechanisms how RNA helicases are targeted to their RNA substrate despite the inherent lack of sequence specificity of their core domains. Additionally, this can lead to unraveling of more structural functions of RNA helicases. A good example is again provided by Dbp5, which interacts with Gle1 and Nup159 to cooperatively to remove export adaptor proteins Mex67 and Nab2 during the transport of mRNA across the nucleoplasm (Hodge et al., 1999; Lund \& Guthrie, 2005; Tran et al., 2007; Weirich et al., 2006). In the context of ribosome assembly pathway, Dbp5 has been reported to interact with Mex67 but using a different transport mechanism (Neumann et al., 2016).

\subsection{Fine-tuning of RNA helicase Function}

As exemplified above, some RNA helicases can be targeted to specific RNAs and/or RNPs and can have multiple functions, with or without the need for the catalytic activity. As described earlier, the $\mathrm{Q}$ domain or ancillary C-terminal domain can target DExD RNA helicases to their substrate and/or regulate the catalytic activity of the protein. For some DExD box RNA helicases with no $\mathrm{N}$ or $\mathrm{C}$-terminal extension, i.e. elF4A-like family, a group of proteins containing a common MIF4G domain can act as cofactors to regulate their activity. For instance, the stimulation of helicase activity of the 'minimal' DEXD box RNA helicases eIFAI/II by the translation initiation factor elF4G1 (Andreou \& Klostermeier, 2014; Hilbert et al., 2011). Interestingly, an inhibitory effect that endows RNA helicases RNA clamping or placeholder function is exemplified by another MIF4G domain-containing protein CWC22, which limits ATP hydrolysis by elF4AIII, an RNA helicase associated with exon-junction complex (Alexandrov et al., 2012; Steckelberg et al., 2012). In a recent study, it has been shown that the ATPase activity of Fal1 is stimulated by Sgd1 and that the interaction between these two proteins are necessary in the early steps of SSU biogenesis (Gallesio et al., 2020). Co-factors of DExD box RNA helicases not belonging to the MIF4G family have also been documented. An example is Esf2, which helps in the recruitment of Dbp8 to pre-ribosomes and also enhances the ATPase activity of the RNA helicase (Granneman et al., 2006). Another example is the mRNA binding protein Yra1 that, in contrast, inhibits the unwinding activity of Dbp2 (W. K. Ma et al., 2013). In the case of Dbp3 and Dbp7, no dedicated co-factors have been reported to date. Furthermore, aside from the scarcity of MIF4G-containing protein identified by MS, our Dbp7 pulldown experiment did not reveal enrichment of a particular protein similar to Dbp7-TAP, indicative of a putative co-factor. However, it is possible that such approach will not evidently identify a 
putative co-factor due to the multitude of AF simultaneously present in pre-ribosomal particles. Structural information of Dbp7- and Dbp3-contaning particle can be useful to identify putative co-factors of these two DExD RNA helicases. In the case of some DEAH box RNA helicases, a family of proteins called the G-patch proteins act as co-factors. For example, in yeast, four G-patch proteins, namely Pfa1, Gno1, Spp382, and Cmg1 have been reported to stimulate the ATPase and/or unwinding activity of Prp43 and are important for Prp43 multifunctionality (Boon et al., 2006; Y.-L. Chen et al., 2014; Heininger et al., 2016; Lebaron et al., 2009; Tanaka et al., 2007).

Aside from co-factors, other modes of regulation have been reported. This include autoregulation, recruitment to specific RNA sequence motifs, regulation by non-substrate RNAs, and post-translational modifications (PTMs) (Sloan \& Bohnsack, 2018). A number of PTMs have been shown to influence the cellular activity of human RNA helicases DDX5, DDX23, and DDX21 (Jacobs et al., 2007; Mathew et al., 2008; Song et al., 2017). PTMs may have impact on the network of protein interactions or directly influence the helicase catalytic activity (Sloan \& Bohnsack, 2018). In the case of Dbp7, a phosphorylation site at S74 reside has been identified and can also be a site for ubiquitination, suggesting a regulatory point for gene expression (Swaney et al., 2013). In a more recent report, phosphorylation on this site is linked to stress response of yeast cells (MacGilvray et al., 2020). The involvement of this phosphorylation site on the ATPase activity and protein interactions of Dbp7 remains to be explored. In the case of Dbp3, no post-translation modification site has been reported to date. Nevertheless, regulatory mechanisms governing its dual function, i.e. in ribosome assembly and snoRNA processing, are worth exploring.

Altogether, the findings of this work expand the portfolio of functionally characterized RNA helicases, particularly those implicated in the early stages of LSU biogenesis. The development of methods for structural investigations and RNA-protein interaction studies will be essential in tackling questions beyond the scope of the study. Nevertheless, this work brings the field one step closer in elucidating the precise functions of the two DEAD box RNA helicases Dbp3 and Dbp7 


\section{Bibliography}

Adams, C. C., Jakovljevic, J., Roman, J., Harnpicharnchai, P., \& Woolford, J. L. (2002). Saccharomyces cerevisiae nucleolar protein Nop7p is necessary for biogenesis of 60 S ribosomal subunits. RNA (New York, N.Y.), 8(2), 150-165.

Aitken, C. E., \& Lorsch, J. R. (2012). A mechanistic overview of translation initiation in eukaryotes. Nature Structural \& Molecular Biology, 19(6), 568-576.

Albert, S., Wietrzynski, W., Lee, C.-W., Schaffer, M., Beck, F., Schuller, J. M., Salomé, P. A., Plitzko, J. M., Baumeister, W., \& Engel, B. D. (2020). Direct visualization of degradation microcompartments at the ER membrane. Proceedings of the National Academy of Sciences, 117(2), 1069-1080.

Alexandrov, A., Colognori, D., Shu, M.-D., \& Steitz, J. A. (2012). Human spliceosomal protein CWC22 plays a role in coupling splicing to exon junction complex deposition and nonsense-mediated decay. Proceedings of the National Academy of Sciences, 109(52), 21313-21318.

Allmang, C., \& Tollervey, D. (1998). The role of the 3' external transcribed spacer in yeast pre-rRNA processing. Journal of Molecular Biology, 278(1), 67-78.

Andersen, C. B. F., Ballut, L., Johansen, J. S., Chamieh, H., Nielsen, K. H., Oliveira, C. L. P., Pedersen, J. S., Séraphin, B., Le Hir, H., \& Andersen, G. R. (2006). Structure of the exon junction core complex with a trapped DEAD-box ATPase bound to RNA. Science (New York, N.Y.), 313(5795), 1968-1972.

Andreou, A. Z., \& Klostermeier, D. (2014). EIF4B and elF4G jointly stimulate elF4A ATPase and unwinding activities by modulation of the elF4A conformational cycle. Journal of Molecular Biology, 426(1), 51-61.

Aravind, L., \& Koonin, E. V. (1999). G-patch: A new conserved domain in eukaryotic RNAprocessing proteins and type $\mathrm{D}$ retroviral polyproteins. Trends in Biochemical Sciences, 24(9), 342-344.

Bachellerie, J. P., Cavaillé, J., \& Hüttenhofer, A. (2002). The expanding snoRNA world. Biochimie, 84(8), 775-790.

Balakin, A. G., Smith, L., \& Fournier, M. J. (1996). The RNA world of the nucleolus: Two major families of small RNAs defined by different box elements with related functions. Cell, 86(5), 823-834.

Ballut, L., Marchadier, B., Baguet, A., Tomasetto, C., Séraphin, B., \& Le Hir, H. (2005). The exon junction core complex is locked onto RNA by inhibition of elF4AIII ATPase activity. Nature Structural \& Molecular Biology, 12(10), 861-869. 
Banroques, J., Cordin, O., Doère, M., Linder, P., \& Tanner, N. K. (2008). A conserved phenylalanine of motif IV in superfamily 2 helicases is required for cooperative, ATPdependent binding of RNA substrates in DEAD-box proteins. Molecular and Cellular Biology, 28(10), 3359-3371.

Barandun, J., Chaker-Margot, M., Hunziker, M., Molloy, K. R., Chait, B. T., \& Klinge, S. (2017). The complete structure of the small-subunit processome. Nature Structural \& Molecular Biology, 24(11), 944-953.

Barrio-Garcia, C., Thoms, M., Flemming, D., Kater, L., Berninghausen, O., Baßler, J., Beckmann, R., \& Hurt, E. (2016). Architecture of the Rix1-Rea1 checkpoint machinery during pre-60S-ribosome remodeling. Nature Structural \& Molecular Biology, 23(1), 37-44.

Baudin-Baillieu, A., Fabret, C., Liang, X.-H., Piekna-Przybylska, D., Fournier, M. J., \& Rousset, J.-P. (2009). Nucleotide modifications in three functionally important regions of the Saccharomyces cerevisiae ribosome affect translation accuracy. Nucleic Acids Research, 37(22), 7665-7677.

Baxter-Roshek, J. L., Petrov, A. N., \& Dinman, J. D. (2007). Optimization of ribosome structure and function by rRNA base modification. PloS One, 2(1), e174.

Bellaousov, S., Kayedkhordeh, M., Peterson, R. J., \& Mathews, D. H. (2018). Accelerated RNA secondary structure design using preselected sequences for helices and loops. $R N A, 24(11), 1555-1567$.

Beltrame, M., Henry, Y., \& Tollervey, D. (1994). Mutational analysis of an essential binding site for the U3 snoRNA in the 5' external transcribed spacer of yeast pre-rRNA. Nucleic Acids Research, 22(23), 5139-5147.

Ben-Shem, A., Loubresse, N. G. de, Melnikov, S., Jenner, L., Yusupova, G., \& Yusupov, M. (2011). The Structure of the Eukaryotic Ribosome at $3.0 \AA$ A Resolution. Science, 334(6062), 1524-1529.

Bernstein, K. A., Granneman, S., Lee, A. V., Manickam, S., \& Baserga, S. J. (2006). Comprehensive Mutational Analysis of Yeast DEXD/H Box RNA Helicases Involved in Large Ribosomal Subunit Biogenesis. Molecular and Cellular Biology, 26(4), 11951208.

Birkedal, U., Christensen-Dalsgaard, M., Krogh, N., Sabarinathan, R., Gorodkin, J., \& Nielsen, $H$. (2015). Profiling of ribose methylations in RNA by high-throughput sequencing. Angewandte Chemie (International Ed. in English), 54(2), 451-455.

Bizebard, T., Ferlenghi, I., lost, I., \& Dreyfus, M. (2004). Studies on three E. coli DEAD-box helicases point to an unwinding mechanism different from that of model DNA helicases. Biochemistry, 43(24), 7857-7866.

Bleichert, F., \& Baserga, S. J. (2007). The Long Unwinding Road of RNA Helicases. Molecular Cell, 27(3), 339-352. 
Bleichert, F., Gagnon, K. T., Brown, B. A., Maxwell, E. S., Leschziner, A. E., Unger, V. M., \& Baserga, S. J. (2009). A dimeric structure for archaeal box C/D small ribonucleoproteins. Science (New York, N.Y.), 325(5946), 1384-1387.

Bohnsack, M. T., Kos, M., \& Tollervey, D. (2008). Quantitative analysis of snoRNA association with pre-ribosomes and release of snR30 by Rok1 helicase. EMBO Reports, 9(12), 1230-1236.

Bohnsack, M. T., Martin, R., Granneman, S., Ruprecht, M., Schleiff, E., \& Tollervey, D. (2009). Prp43 Bound at Different Sites on the Pre-rRNA Performs Distinct Functions in Ribosome Synthesis. Molecular Cell, 36(4-24), 583-592.

Boon, K.-L., Auchynnikava, T., Edwalds-Gilbert, G., Barrass, J. D., Droop, A. P., Dez, C., \& Beggs, J. D. (2006). Yeast Ntr1/Spp382 Mediates Prp43 Function in Postspliceosomes. Molecular and Cellular Biology, 26(16), 6016-6023.

Brimacombe, R., Mitchell, P., Osswald, M., Stade, K., \& Bochkariov, D. (1993). Clustering of modified nucleotides at the functional center of bacterial ribosomal RNA. FASEB Journal: Official Publication of the Federation of American Societies for Experimental Biology, 7(1), 161-167.

Brüning, L., Hackert, P., Martin, R., Gallesio, J. D., Aquino, G. R. R., Urlaub, H., Sloan, K. E., \& Bohnsack, M. T. (2018). RNA helicases mediate structural transitions and compositional changes in pre-ribosomal complexes. Nature Communications, 9(1), 5383.

Buchhaupt, M., Sharma, S., Kellner, S., Oswald, S., Paetzold, M., Peifer, C., Watzinger, P., Schrader, J., Helm, M., \& Entian, K.-D. (2014). Partial methylation at Am100 in 18S rRNA of baker's yeast reveals ribosome heterogeneity on the level of eukaryotic rRNA modification. PloS One, 9(2), e89640.

Burger, F., Daugeron, M.-C., \& Linder, P. (2000). Dbp10p, a putative RNA helicase from Saccharomyces cerevisiae, is required for ribosome biogenesis. Nucleic Acids Research, 28(12), 2315-2323.

Cahill, N. M., Friend, K., Speckmann, W., Li, Z.-H., Terns, R. M., Terns, M. P., \& Steitz, J. A. (2002). Site-specific cross-linking analyses reveal an asymmetric protein distribution for a box C/D snoRNP. The EMBO Journal, 21(14), 3816-3828.

Caruthers, J. M., \& McKay, D. B. (2002). Helicase structure and mechanism. Current Opinion in Structural Biology, 12(1), 123-133.

Chen, M. C., Murat, P., Abecassis, K., Ferré-D’Amaré, A. R., \& Balasubramanian, S. (2015). Insights into the mechanism of a G-quadruplex-unwinding DEAH-box helicase. Nucleic Acids Research, 43(4), 2223-2231. 
Chen, M. C., Tippana, R., Demeshkina, N. A., Murat, P., Balasubramanian, S., Myong, S., \& Ferré-D'Amaré, A. R. (2018). Structural basis of G-quadruplex unfolding by the DEAH/RHA helicase DHX36. Nature, 558(7710), 465-469.

Chen, Y., Potratz, J. P., Tijerina, P., Del Campo, M., Lambowitz, A. M., \& Russell, R. (2008). DEAD-box proteins can completely separate an RNA duplex using a single ATP. Proceedings of the National Academy of Sciences of the United States of America, 105(51), 20203-20208.

Chen, Y.-L., Capeyrou, R., Humbert, O., Mouffok, S., Kadri, Y. A., Lebaron, S., Henras, A. K., \& Henry, Y. (2014). The telomerase inhibitor Gno1p/PINX1 activates the helicase Prp43p during ribosome biogenesis. Nucleic Acids Research, 42(11), 7330-7345.

Choudhury, P., Hackert, P., Memet, I., Sloan, K. E., \& Bohnsack, M. T. (2019). The human RNA helicase DHX37 is required for release of the U3 snoRNP from pre-ribosomal particles. RNA Biology, 16(1), 54-68.

Choudhury, P., Kretschmer, J., Hackert, P., Bohnsack, K. E., \& Bohnsack, M. T. (2020). The DExD box ATPase DDX55 is recruited to domain IV of the 28S ribosomal RNA by its C-terminal region. RNA Biology, O(0), 1-12.

Chu, S., Archer, R. H., Zengel, J. M., \& Lindahl, L. (1994). The RNA of RNase MRP is required for normal processing of ribosomal RNA. Proceedings of the National Academy of Sciences of the United States of America, 91(2), 659-663.

Cordin, O., Banroques, J., Tanner, N. K., \& Linder, P. (2006). The DEAD-box protein family of RNA helicases. Gene, 367, 17-37.

Cordin, O., \& Beggs, J. D. (2013). RNA helicases in splicing. RNA Biology, 10(1), 83-95.

Cordin, O., Tanner, N. K., Doère, M., Linder, P., \& Banroques, J. (2004). The newly discovered $Q$ motif of DEAD-box RNA helicases regulates RNA-binding and helicase activity. The EMBO Journal, 23(13), 2478-2487.

Daugeron, M. C., Kressler, D., \& Linder, P. (2001). Dbp9p, a putative ATP-dependent RNA helicase involved in 60S-ribosomal-subunit biogenesis, functionally interacts with Dbp6p. RNA, 7(9), 1317-1334.

Daugeron, M. C., \& Linder, P. (1998). Dbp7p, a putative ATP-dependent RNA helicase from Saccharomyces cerevisiae, is required for 605 ribosomal subunit assembly. $R N A$ (New York, N.Y.), 4(5), 566-581.

Davis, D. R. (1995). Stabilization of RNA stacking by pseudouridine. Nucleic Acids Research, 23(24), 5020-5026.

Davis, J. H., Tan, Y. Z., Carragher, B., Potter, C. S., Lyumkis, D., \& Williamson, J. R. (2016). Modular Assembly of the Bacterial Large Ribosomal Subunit. Cell, 167(6), 16101622.e15. 
de la Cruz, J., Gómez-Herreros, F., Rodríguez-Galán, O., Begley, V., de la Cruz MuñozCenteno, M., \& Chávez, S. (2018). Feedback regulation of ribosome assembly. Current Genetics, 64(2), 393-404.

de la Cruz, J., Karbstein, K., \& Woolford, J. L. (2015). Functions of ribosomal proteins in assembly of eukaryotic ribosomes in vivo. Annual Review of Biochemistry, 84, 93129.

de la Cruz, J., Lacombe, T., Deloche, O., Linder, P., \& Kressler, D. (2004). The putative RNA helicase Dbp6p functionally interacts with Rpl3p, Nop8p and the novel trans-acting Factor Rsa3p during biogenesis of 60S ribosomal subunits in Saccharomyces cerevisiae. Genetics, 166(4), 1687-1699.

Decatur, W. A., \& Fournier, M. J. (2002). RRNA modifications and ribosome function. Trends in Biochemical Sciences, 27(7), 344-351.

Del Campo, M., \& Lambowitz, A. M. (2009). Structure of the Yeast DEAD box protein Mss116p reveals two wedges that crimp RNA. Molecular Cell, 35(5), 598-609.

Dembowski, J. A., Kuo, B., \& Woolford, J. L. (2013). Has1 regulates consecutive maturation and processing steps for assembly of 605 ribosomal subunits. Nucleic Acids Research, 41(16), 7889-7904.

Dembowski, J. A., Ramesh, M., McManus, C. J., \& Woolford, J. L. (2013). Identification of the binding site of Rlp7 on assembling 60S ribosomal subunits in Saccharomyces cerevisiae. RNA, 19(12), 1639-1647.

Dez, C., Froment, C., Noaillac-Depeyre, J., Monsarrat, B., Caizergues-Ferrer, M., \& Henry, Y. (2004). Npa1p, a component of very early pre-60S ribosomal particles, associates with a subset of small nucleolar RNPs required for peptidyl transferase center modification. Molecular and Cellular Biology, 24(14), 6324-6337.

Dillingham, M. S., Soultanas, P., \& Wigley, D. B. (1999). Site-directed mutagenesis of motif III in PcrA helicase reveals a role in coupling ATP hydrolysis to strand separation. Nucleic Acids Research, 27(16), 3310-3317.

Donsbach, P., Yee, B. A., Sanchez-Hevia, D., Berenguer, J., Aigner, S., Yeo, G. W., \& Klostermeier, D. (2020). The Thermus thermophilus DEAD-box protein Hera is a general RNA binding protein and plays a key role in tRNA metabolism. RNA, rna.075580.120.

Fabian, G. R., \& Hopper, A. K. (1987). RRP1, a Saccharomyces cerevisiae gene affecting rRNA processing and production of mature ribosomal subunits. Journal of Bacteriology, 169(4), 1571-1578.

Fairman-Williams, M. E., Guenther, U.-P., \& Jankowsky, E. (2010). SF1 and SF2 helicases: Family matters. Current Opinion in Structural Biology, 20(3), 313-324. 
Falk, S., Tants, J.-N., Basquin, J., Thoms, M., Hurt, E., Sattler, M., \& Conti, E. (2017). Structural insights into the interaction of the nuclear exosome helicase Mtr4 with the preribosomal protein Nop53. RNA, 23(12), 1780-1787.

Fatica, A., Oeffinger, M., Dlakić, M., \& Tollervey, D. (2003a). Nob1p is required for cleavage of the 3' end of 18S rRNA. Molecular and Cellular Biology, 23(5), 1798-1807.

Fatica, A., Oeffinger, M., Tollervey, D., \& Bozzoni, I. (2003b). Cic1p/Nsa3p is required for synthesis and nuclear export of $60 \mathrm{~S}$ ribosomal subunits. RNA (New York, N.Y.), 9(12), 1431-1436.

Fitzgerald, M. E., Rawling, D. C., Vela, A., \& Pyle, A. M. (2014). An evolving arsenal: Viral RNA detection by RIG-I-like receptors. Current Opinion in Microbiology, 20, 76-81.

Gadal, O., Strauss, D., Petfalski, E., Gleizes, P.-E., Gas, N., Tollervey, D., \& Hurt, E. (2002). RIp7p is associated with 60S preribosomes, restricted to the granular component of the nucleolus, and required for pre-rRNA processing. The Journal of Cell Biology, 157(6), 941-951.

Gallesio, J. D., Hackert, P., Bohnsack, K. E., \& Bohnsack, M. T. (2020). Sgd1 is an MIF4G domain-containing cofactor of the RNA helicase Fal1 and associates with the 5' domain of the 18S rRNA sequence. RNA Biology, 17(4), 539-553.

Gamalinda, M., Ohmayer, U., Jakovljevic, J., Kumcuoglu, B., Woolford, J., Mbom, B., Lin, L., \& Woolford, J. L. (2014). A hierarchical model for assembly of eukaryotic $60 \mathrm{~S}$ ribosomal subunit domains. Genes \& Development, 28(2), 198-210.

Garcia, I., Albring, M. J., \& Uhlenbeck, O. C. (2012). Duplex Destabilization by Four Ribosomal DEAD-Box Proteins. Biochemistry, 51(50), 10109-10118.

García-García, C., Frieda, K. L., Feoktistova, K., Fraser, C. S., \& Block, S. M. (2015). Factordependent processivity in human elF4A DEAD-box helicase. Science, 348(6242), 1486-1488.

Geerlings, T. H., Vos, J. C., \& Raué, H. A. (2000). The final step in the formation of 25S rRNA in Saccharomyces cerevisiae is performed by $5 \rightarrow 3$ ' exonucleases. RNA, 6(12), 1698-1703.

Gerbi, S. A. (1986). The evolution of eukaryotic ribosomal DNA. Bio Systems, 19(4), 247258.

Gerhardy, S., Menet, A. M., Peña, C., Petkowski, J. J., \& Panse, V. G. (2014). Assembly and nuclear export of pre-ribosomal particles in budding yeast. Chromosoma, 123(4), 327-344.

Gigova, A., Duggimpudi, S., Pollex, T., Schaefer, M., \& Koš, M. (2014). A cluster of methylations in the domain IV of 25S rRNA is required for ribosome stability. RNA (New York, N.Y.), 20(10), 1632-1644. 
Gilman, B., Tijerina, P., \& Russell, R. (2017). Distinct RNA unwinding mechanisms of DEADbox and DEAH-box RNA helicase proteins in remodeling structured RNAs and RNPs. Biochemical Society Transactions, 45(6), 1313.

Gnanasundram, S. V., Kos-Braun, I. C., \& Koš, M. (2019). At least two molecules of the RNA helicase Has1 are simultaneously present in pre-ribosomes during ribosome biogenesis. Nucleic Acids Research, 47(20), 10852-10864.

Gorbalenya, A. E., \& Koonin, E. V. (1993). Helicases: Amino acid sequence comparisons and structure-function relationships. Current Opinion in Structural Biology, 3(3), 419429.

Granneman, S., Bernstein, K. A., Bleichert, F., \& Baserga, S. J. (2006). Comprehensive Mutational Analysis of Yeast DEXD/H Box RNA Helicases Required for Small Ribosomal Subunit Synthesis. Molecular and Cellular Biology, 26(4), 1183-1194.

Granneman, S., Petfalski, E., \& Tollervey, D. (2011). A cluster of ribosome synthesis factors regulate pre-rRNA folding and 5.8S rRNA maturation by the Rat1 exonuclease. The EMBO Journal, 30(19), 4006-4019.

Greber, B. J. (2016). Mechanistic insight into eukaryotic 60S ribosomal subunit biogenesis by cryo-electron microscopy. RNA (New York, N.Y.), 22(11), 1643-1662.

Grohman, J. K., Del Campo, M., Bhaskaran, H., Tijerina, P., Lambowitz, A. M., \& Russell, R. (2007). Probing the Mechanisms of DEAD-Box Proteins as General RNA Chaperones: The C-Terminal Domain of CYT-19 Mediates General Recognition of RNA. Biochemistry, 46(11), 3013-3022.

Guglielmi, B., \& Werner, M. (2002). The Yeast Homolog of Human PinX1 Is Involved in rRNA and Small Nucleolar RNA Maturation, Not in Telomere Elongation Inhibition. Journal of Biological Chemistry, 277(38), 35712-35719.

Gupta, N., Lorsch, J. R., \& Hinnebusch, A. G. (2018). Yeast Ded1 promotes 48S translation pre-initiation complex assembly in an mRNA-specific and elF4F-dependent manner. ELife, 7, e38892.

Haag, S., Kretschmer, J., \& Bohnsack, M. T. (2015). WBSCR22/Merm1 is required for late nuclear pre-ribosomal RNA processing and mediates N7-methylation of G1639 in human 18S rRNA. RNA, 21(2), 180-187.

Halbach, F., Reichelt, P., Rode, M., \& Conti, E. (2013). The Yeast Ski Complex: Crystal Structure and RNA Channeling to the Exosome Complex. Cell, 154(4), 814-826.

Hamann, F., Enders, M., \& Ficner, R. (2019). Structural basis for RNA translocation by DEAH-box ATPases. Nucleic Acids Research, 47(8), 4349-4362.

Hamann, F., Schmitt, A., Favretto, F., Hofele, R., Neumann, P., Xiang, S., Urlaub, H., Zweckstetter, M., \& Ficner, R. (2020). Structural analysis of the intrinsically 
disordered splicing factor Spp2 and its binding to the DEAH-box ATPase Prp2. Proceedings of the National Academy of Sciences, 117(6), 2948-2956.

Hardin, J. W., Hu, Y. X., \& McKay, D. B. (2010). Structure of the RNA Binding Domain of a DEAD-Box Helicase Bound to Its Ribosomal RNA Target Reveals a Novel Mode of Recognition by an RNA Recognition Motif. Journal of Molecular Biology, 402(2), 412427.

He, Y., Andersen, G. R., \& Nielsen, K. H. (2010). Structural basis for the function of DEAH helicases. EMBO Reports, 11(3), 180-186.

Heininger, A. U., Hackert, P., Andreou, A. Z., Boon, K.-L., Memet, I., Prior, M., Clancy, A., Schmidt, B., Urlaub, H., Schleiff, E., Sloan, K. E., Deckers, M., Lührmann, R., Enderlein, J., Klostermeier, D., Rehling, P., \& Bohnsack, M. T. (2016). Protein cofactor competition regulates the action of a multifunctional RNA helicase in different pathways. RNA Biology, 13(3), 320-330.

Helm, M. (2006). Post-transcriptional nucleotide modification and alternative folding of RNA. Nucleic Acids Research, 34(2), 721-733.

Henn, A., Cao, W., Licciardello, N., Heitkamp, S. E., Hackney, D. D., \& De La Cruz, E. M. (2010). Pathway of ATP utilization and duplex rRNA unwinding by the DEAD-box helicase, DbpA. Proceedings of the National Academy of Sciences of the United States of America, 107(9), 4046-4050.

Henras, A. K., Plisson-Chastang, C., Humbert, O., Romeo, Y., \& Henry, Y. (2017). Synthesis, Function, and Heterogeneity of snoRNA-Guided Posttranscriptional Nucleoside Modifications in Eukaryotic Ribosomal RNAs. The Enzymes, 41, 169-213.

Henras, A. K., Plisson-Chastang, C., O'Donohue, M.-F., Chakraborty, A., \& Gleizes, P.-E. (2015). An overview of pre-ribosomal RNA processing in eukaryotes. Wiley Interdisciplinary Reviews. RNA, 6(2), 225-242.

Henry, Y., Wood, H., Morrissey, J. P., Petfalski, E., Kearsey, S., \& Tollervey, D. (1994). The 5 ' end of yeast $5.8 S$ rRNA is generated by exonucleases from an upstream cleavage site. The EMBO Journal, 13(10), 2452-2463.

Hierlmeier, T., Merl, J., Sauert, M., Perez-Fernandez, J., Schultz, P., Bruckmann, A., Hamperl, S., Ohmayer, U., Rachel, R., Jacob, A., Hergert, K., Deutzmann, R., Griesenbeck, J., Hurt, E., Milkereit, P., Baßler, J., \& Tschochner, H. (2013). Rrp5p, Noc1p and Noc2p form a protein module which is part of early large ribosomal subunit precursors in S. cerevisiae. Nucleic Acids Research, 41(2), 1191-1210.

Hilbert, M., Karow, A. R., \& Klostermeier, D. (2009). The mechanism of ATP-dependent RNA unwinding by DEAD box proteins. Biological Chemistry, 390(12), 1237-1250.

Hilbert, M., Kebbel, F., Gubaev, A., \& Klostermeier, D. (2011). EIF4G stimulates the activity of the DEAD box protein elF4A by a conformational guidance mechanism. Nucleic Acids Research, 39(6), 2260-2270. 
Hilliker, A., Gao, Z., Jankowsky, E., \& Parker, R. (2011). The DEAD-Box Protein Ded1 Modulates Translation by the Formation and Resolution of an elF4F-mRNA Complex. Molecular Cell, 43(6), 962-972.

Hodge, C. A., Colot, H. V., Stafford, P., \& Cole, C. N. (1999). Rat8p/Dbp5p is a shuttling transport factor that interacts with Rat7p/Nup159p and Gle1p and suppresses the mRNA export defect of xpo1-1 cells. The EMBO Journal, 18(20), 5778-5788.

Huang, H.-R., Rowe, C. E., Mohr, S., Jiang, Y., Lambowitz, A. M., \& Perlman, P. S. (2005). The splicing of yeast mitochondrial group I and group II introns requires a DEAD-box protein with RNA chaperone function. Proceedings of the National Academy of Sciences, 102(1), 163-168.

Hughes, J. M., \& Ares, M. (1991). Depletion of U3 small nucleolar RNA inhibits cleavage in the 5' external transcribed spacer of yeast pre-ribosomal RNA and impairs formation of $18 \mathrm{~S}$ ribosomal RNA. The EMBO Journal, 10(13), 4231-4239.

Jack, K., Bellodi, C., Landry, D. M., Niederer, R. O., Meskauskas, A., Musalgaonkar, S., Kopmar, N., Krasnykh, O., Dean, A. M., Thompson, S. R., Ruggero, D., \& Dinman, J. D. (2011). RRNA pseudouridylation defects affect ribosomal ligand binding and translational fidelity from yeast to human cells. Molecular Cell, 44(4), 660-666.

Jacobs, A.-M., Nicol, S. M., Hislop, R. G., Jaffray, E. G., Hay, R. T., \& Fuller-Pace, F. V. (2007). SUMO modification of the DEAD box protein p68 modulates its transcriptional activity and promotes its interaction with HDAC1. Oncogene, 26(40), 5866-5876.

Jäger, S., Strayle, J., Heinemeyer, W., \& Wolf, D. H. (2001). Cic1, an adaptor protein specifically linking the $26 \mathrm{~S}$ proteasome to its substrate, the SCF component Cdc4. The EMBO Journal, 20(16), 4423-4431.

Jankowsky, E. (2011). RNA Helicases at work: Binding and rearranging. Trends in Biochemical Sciences, 36(1), 19-29.

Jankowsky, E., \& Fairman-Williams, M. E. (2010). Chapter 1:An Introduction to RNA Helicases: Superfamilies, Families, and Major Themes. In Chapter 1:An Introduction to RNA Helicases: Superfamilies, Families, and Major Themes (pp. 1-31).

Jarmoskaite, I., \& Russell, R. (2011). DEAD-box proteins as RNA helicases and chaperones. Wiley Interdisciplinary Reviews. RNA, 2(1), 135-152.

Jarmoskaite, I., \& Russell, R. (2014). RNA Helicase Proteins as Chaperones and Remodelers. Annual Review of Biochemistry, 83(1), 697-725.

Jeske, M., Müller, C. W., \& Ephrussi, A. (2017). The LOTUS domain is a conserved DEADbox RNA helicase regulator essential for the recruitment of Vasa to the germ plasm and nuage. Genes \& Development, 31(9), 939-952. 
Jia, H., Wang, X., Anderson, J. T., \& Jankowsky, E. (2012). RNA unwinding by the Trf4/Air2/Mtr4 polyadenylation (TRAMP) complex. Proceedings of the National Academy of Sciences, 109(19), 7292-7297.

Joret, C., Capeyrou, R., Belhabich-Baumas, K., Plisson-Chastang, C., Ghandour, R., Humbert, O., Fribourg, S., Leulliot, N., Lebaron, S., Henras, A. K., \& Henry, Y. (2018). The Npa1p complex chaperones the assembly of the earliest eukaryotic large ribosomal subunit precursor. PLOS Genetics, 14(8), e1007597.

Kargas, V., Castro-Hartmann, P., Escudero-Urquijo, N., Dent, K., Hilcenko, C., Sailer, C., Zisser, G., Marques-Carvalho, M. J., Pellegrino, S., Wawiórka, L., Freund, S. M., Wagstaff, J. L., Andreeva, A., Faille, A., Chen, E., Stengel, F., Bergler, H., \& Warren, A. J. (2019). Mechanism of completion of peptidyltransferase centre assembly in eukaryotes. ELife, 8, e44904.

Karginov, F. V., Caruthers, J. M., Hu, Y., McKay, D. B., \& Uhlenbeck, O. C. (2005). YxiN Is a Modular Protein Combining a DExD/H Core and a Specific RNA-binding Domain. Journal of Biological Chemistry, 280(42), 35499-35505.

Kater, L., Thoms, M., Barrio-Garcia, C., Cheng, J., Ismail, S., Ahmed, Y. L., Bange, G., Kressler, D., Berninghausen, O., Sinning, I., Hurt, E., \& Beckmann, R. (2017). Visualizing the Assembly Pathway of Nucleolar Pre-60S Ribosomes. Cell, 171(7), 1599-1610.e14.

Khatter, H., Myasnikov, A. G., Natchiar, S. K., \& Klaholz, B. P. (2015). Structure of the human 80 s ribosome. Nature, 520(7549), 640-645.

Khoshnevis, S., Askenasy, I., Johnson, M. C., Dattolo, M. D., Young-Erdos, C. L., Stroupe, M. E., \& Karbstein, K. (2016). The DEAD-box Protein Rok1 Orchestrates 40S and 60S Ribosome Assembly by Promoting the Release of Rrp5 from Pre-40S Ribosomes to Allow for 60S Maturation. PLOS Biology, 14(6), e1002480.

Kilchert, C., Wittmann, S., \& Vasiljeva, L. (2016). The regulation and functions of the nuclear RNA exosome complex. Nature Reviews Molecular Cell Biology, 17(4), 227-239.

King, T. H., Liu, B., McCully, R. R., \& Fournier, M. J. (2003). Ribosome structure and activity are altered in cells lacking snoRNPs that form pseudouridines in the peptidyl transferase center. Molecular Cell, 11(2), 425-435.

Kiss, T., Fayet-Lebaron, E., \& Jády, B. E. (2010). Box H/ACA small ribonucleoproteins. Molecular Cell, 37(5), 597-606. https://doi.org/10.1016/j.molcel.2010.01.032

Kiss-László, Z., Henry, Y., Bachellerie, J. P., Caizergues-Ferrer, M., \& Kiss, T. (1996). Sitespecific ribose methylation of preribosomal RNA: A novel function for small nucleolar RNAs. Cell, 85(7), 1077-1088.

Kiss-László, Z., Henry, Y., \& Kiss, T. (1998). Sequence and structural elements of methylation guide snoRNAs essential for site-specific ribose methylation of pre-rRNA. The EMBO Journal, 17(3), 797-807. 
Klingauf-Nerurkar, P., Gillet, L. C., Portugal-Calisto, D., Oborská-Oplová, M., Jäger, M., Schubert, O. T., Pisano, A., Peña, C., Rao, S., Altvater, M., Chang, Y., Aebersold, R., \& Panse, V. G. (2020). The GTPase Nog1 co-ordinates the assembly, maturation and quality control of distant ribosomal functional centers. ELife, 9, e52474.

Klinge, S., Voigts-Hoffmann, F., Leibundgut, M., \& Ban, N. (2012). Atomic structures of the eukaryotic ribosome. Trends in Biochemical Sciences, 37(5), 189-198.

Klinge, S., \& Woolford, J. L. (2019). Ribosome assembly coming into focus. Nature Reviews Molecular Cell Biology, 20(2), 116-131.

Klostermeier, D., \& Rudolph, M. G. (2009). A novel dimerization motif in the C-terminal domain of the Thermus thermophilus DEAD box helicase Hera confers substantial flexibility. Nucleic Acids Research, 37(2), 421.

Konikkat, S., \& Woolford, J. L. (2017). Principles of 60 S ribosomal subunit assembly emerging from recent studies in yeast. The Biochemical Journal, 474(2), 195-214.

Kornprobst, M., Turk, M., Kellner, N., Cheng, J., Flemming, D., Koš-Braun, I., Koš, M., Thoms, M., Berninghausen, O., Beckmann, R., \& Hurt, E. (2016). Architecture of the 90S Pre-ribosome: A Structural View on the Birth of the Eukaryotic Ribosome. Cell, 166(2), 380-393.

Kos, M., \& Tollervey, D. (2005). The Putative RNA Helicase Dbp4p Is Required for Release of the U14 snoRNA from Preribosomes in Saccharomyces cerevisiae. Molecular Cell, 20(1), 53-64.

Kos, M., \& Tollervey, D. (2010). Yeast pre-rRNA processing and modification occur cotranscriptionally. Molecular Cell, 37(6), 809-820.

Kramer, K., Sachsenberg, T., Beckmann, B. M., Qamar, S., Boon, K.-L., Hentze, M. W., Kohlbacher, O., \& Urlaub, H. (2014). Photo-cross-linking and high-resolution mass spectrometry for assignment of RNA-binding sites in RNA-binding proteins. Nature Methods, 11(10), 1064-1070.

Kressler, D., de la Cruz, J., Rojo, M., \& Linder, P. (1998). Dbp6p Is an Essential Putative ATP-Dependent RNA Helicase Required for 60S-Ribosomal-Subunit Assembly in Saccharomyces cerevisiae. Molecular and Cellular Biology, 18(4), 1855-1865.

Kressler, D., Hurt, E., \& Bassler, J. (2010). Driving ribosome assembly. Biochimica Et Biophysica Acta, 1803(6), 673-683.

Kressler, D., Linder, P., \& Cruz, J. de la. (1999). Protein trans-Acting Factors Involved in Ribosome Biogenesis in Saccharomyces cerevisiae. Molecular and Cellular Biology, 19(12), 7897-7912.

Krogh, N., Birkedal, U., \& Nielsen, H. (2017). RiboMeth-seq: Profiling of 2'-O-Me in RNA. Methods in Molecular Biology (Clifton, N.J.), 1562, 189-209. 
Krogh, N., \& Nielsen, H. (2019). Sequencing-based methods for detection and quantitation of ribose methylations in RNA. Methods (San Diego, Calif.), 156, 5-15.

Kuba, J., Mitchels, J., Hovorka, M., Erdmann, P., Berka, L., Kirmse, R., KÖnig, J., DE Bock, J., Goetze, B., \& Rigort, A. (2020). Advanced cryo-tomography workflow developments-Correlative microscopy, milling automation and cryo-lift-out. Journal of Microscopy.

Kudla, G., Granneman, S., Hahn, D., Beggs, J. D., \& Tollervey, D. (2011). Cross-linking, ligation, and sequencing of hybrids reveals RNA-RNA interactions in yeast. Proceedings of the National Academy of Sciences of the United States of America, 108(24), 10010-10015.

Lafontaine, D. L., Bousquet-Antonelli, C., Henry, Y., Caizergues-Ferrer, M., \& Tollervey, D. (1998). The box H + ACA snoRNAs carry Cbf5p, the putative rRNA pseudouridine synthase. Genes \& Development, 12(4), 527-537.

Lafontaine, D., Vandenhaute, J., \& Tollervey, D. (1995). The 18S rRNA dimethylase Dim1p is required for pre-ribosomal RNA processing in yeast. Genes \& Development, 9(20), 2470-2481.

Lattmann, S., Giri, B., Vaughn, J. P., Akman, S. A., \& Nagamine, Y. (2010). Role of the amino terminal RHAU-specific motif in the recognition and resolution of guanine quadruplex-RNA by the DEAH-box RNA helicase RHAU. Nucleic Acids Research, $38(18), 6219$.

Lebaron, S., Papin, C., Capeyrou, R., Chen, Y.-L., Froment, C., Monsarrat, B., CaizerguesFerrer, M., Grigoriev, M., \& Henry, Y. (2009). The ATPase and helicase activities of Prp43p are stimulated by the G-patch protein Pfa1p during yeast ribosome biogenesis. The EMBO Journal, 28(24), 3808-3819.

Lebaron, S., Segerstolpe, Å., French, S. L., Dudnakova, T., de lima Alves, F., Granneman, S., Rappsilber, J., Beyer, A. L., Wieslander, L., \& Tollervey, D. (2013). Rrp5 Binding at Multiple Sites Coordinates Pre-rRNA Processing and Assembly. Molecular Cell, 52(5), 707-719.

Leeds, N. B., Small, E. C., Hiley, S. L., Hughes, T. R., \& Staley, J. P. (2006). The Splicing Factor Prp43p, a DEAH Box ATPase, Functions in Ribosome Biogenesis. Molecular and Cellular Biology, 26(2), 513-522.

Li, L., \& Ye, K. (2006). Crystal structure of an H/ACA box ribonucleoprotein particle. Nature, 443(7109), 302-307.

Liang, X., Liu, Q., \& Fournier, M. J. (2009). Loss of rRNA modifications in the decoding center of the ribosome impairs translation and strongly delays pre-rRNA processing. RNA, 15(9), 1716-1728.

Liang, X., \& Fournier, M. J. (2006). The helicase Has1p is required for snoRNA release from pre-rRNA. Molecular and Cellular Biology, 26(20), 7437-7450. 
Lin, J., Lai, S., Jia, R., Xu, A., Zhang, L., Lu, J., \& Ye, K. (2011). Structural basis for sitespecific ribose methylation by box C/D RNA protein complexes. Nature, 469(7331), 559-563.

Lindahl, L., Bommankanti, A., Li, X., Hayden, L., Jones, A., Khan, M., Oni, T., \& Zengel, J. M. (2009). RNase MRP is required for entry of $35 S$ precursor rRNA into the canonical processing pathway. RNA (New York, N.Y.), 15(7), 1407-1416.

Linden, A., Deckers, M., Parfentev, I., Pflanz, R., Homberg, B., Neumann, P., Ficner, R., Rehling, P., \& Urlaub, H. (2020). A Cross-linking Mass Spectrometry Approach Defines Protein Interactions in Yeast Mitochondria. Molecular \& Cellular Proteomics: MCP, 19(7), 1161-1178.

Linder, P., \& Jankowsky, E. (2011). From unwinding to clamping-The DEAD box RNA helicase family. Nature Reviews Molecular Cell Biology, 12(8), 505-516.

Liu, F., Putnam, A., \& Jankowsky, E. (2008). ATP hydrolysis is required for DEAD-box protein recycling but not for duplex unwinding. Proceedings of the National Academy of Sciences of the United States of America, 105(51), 20209-20214.

Liu, Y.-C., \& Cheng, S.-C. (2015). Functional roles of DExD/H-box RNA helicases in PremRNA splicing. Journal of Biomedical Science, 22(1).

Lohman, T. M., \& Bjornson, K. P. (1996). Mechanisms of helicase-catalyzed dna unwinding. Annual Review of Biochemistry, 65(1), 169-214.

Long, E. O., \& Dawid, I. B. (1980). Repeated genes in eukaryotes. Annual Review of Biochemistry, 49, 727-764.

Lund, M. K., \& Guthrie, C. (2005). The DEAD-Box Protein Dbp5p Is Required to Dissociate Mex67p from Exported mRNPs at the Nuclear Rim. Molecular Cell, 20(4), 645-651.

Luo, D., Ding, S. C., Vela, A., Kohlway, A., Lindenbach, B. D., \& Pyle, A. M. (2011). Structural insights into RNA recognition by RIG-I. Cell, 147(2), 409-422.

Lygerou, Z., Allmang, C., Tollervey, D., \& Séraphin, B. (1996). Accurate processing of a eukaryotic precursor ribosomal RNA by ribonuclease MRP in vitro. Science (New York, N.Y.), 272(5259), 268-270.

Ma, C., Wu, S., Li, N., Chen, Y., Yan, K., Li, Z., Zheng, L., Lei, J., Woolford, J. L., \& Gao, N. (2017). Structural snapshot of cytoplasmic pre-60S ribosomal particles bound by Nmd3, Lsg1, Tif6 and Reh1. Nature Structural \& Molecular Biology, 24(3), 214-220.

Ma, W. K., Cloutier, S. C., \& Tran, E. J. (2013). The DEAD-box Protein Dbp2 Functions with the RNA-binding Protein Yra1 to Promote mRNP Assembly. Journal of Molecular Biology, 425(20), 3824-3838. 
MacGilvray, M. E., Shishkova, E., Place, M., Wagner, E. R., Coon, J. J., \& Gasch, A. P. (2020). The phosphoproteome response to dithiothreitol reveals unique versus shared features of Saccharomyces cerevisiae stress responses. Journal of Proteome Research, 19(8), 3405-3417.

Mallam, A. L., Del Campo, M., Gilman, B., Sidote, D. J., \& Lambowitz, A. M. (2012). Structural basis for RNA-duplex recognition and unwinding by the DEAD-box helicase Mss116p. Nature, 490(7418), 121-125.

Mallam, A. L., Jarmoskaite, I., Tijerina, P., Del Campo, M., Seifert, S., Guo, L., Russell, R., \& Lambowitz, A. M. (2011). Solution structures of DEAD-box RNA chaperones reveal conformational changes and nucleic acid tethering by a basic tail. Proceedings of the National Academy of Sciences of the United States of America, 108(30), 1225412259.

Malyutin, A. G., Musalgaonkar, S., Patchett, S., Frank, J., \& Johnson, A. W. (2017). Nmd3 is a structural mimic of eIF5A, and activates the cpGTPase Lsg1 during $60 \mathrm{~S}$ ribosome biogenesis. The EMBO Journal, 36(7), 854-868.

Manikas, R.-G., Thomson, E., Thoms, M., \& Hurt, E. (2016). The K+-dependent GTPase Nug1 is implicated in the association of the helicase Dbp10 to the immature peptidyl transferase centre during ribosome maturation. Nucleic Acids Research, 44(4), 18001812.

Martin R (2014) Functional characterisation of ribosome biogenesis cofactors on Saccharomyces cerevisiae. PhD thesis, Georg-August-Universität Göttingen.

Martin, R., Hackert, P., Ruprecht, M., Simm, S., Brüning, L., Mirus, O., Sloan, K. E., Kudla, G., Schleiff, E., \& Bohnsack, M. T. (2014). A pre-ribosomal RNA interaction network involving snoRNAs and the Rok1 helicase. RNA, 20(8), 1173-1182.

Martin, R., Straub, A. U., Doebele, C., \& Bohnsack, M. T. (2013). DExD/H-box RNA helicases in ribosome biogenesis. RNA Biology, 10(1), 4-18.

Mathew, R., Hartmuth, K., Möhlmann, S., Urlaub, H., Ficner, R., \& Lührmann, R. (2008). Phosphorylation of human PRP28 by SRPK2 is required for integration of the U4/U6U5 tri-snRNP into the spliceosome. Nature Structural \& Molecular Biology, 15(5), 435-443.

Melnikov, S., Ben-Shem, A., Garreau de Loubresse, N., Jenner, L., Yusupova, G., \& Yusupov, M. (2012). One core, two shells: Bacterial and eukaryotic ribosomes. Nature Structural \& Molecular Biology, 19(6), 560-567.

Meyer, B., Wurm, J. P., Kötter, P., Leisegang, M. S., Schilling, V., Buchhaupt, M., Held, M., Bahr, U., Karas, M., Heckel, A., Bohnsack, M. T., Wöhnert, J., \& Entian, K.-D. (2011). The Bowen-Conradi syndrome protein Nep1 (Emg1) has a dual role in eukaryotic ribosome biogenesis, as an essential assembly factor and in the methylation of $\Psi 1191$ in yeast 18S rRNA. Nucleic Acids Research, 39(4), 1526-1537. 
Miller, O. L., \& Beatty, B. R. (1969). Visualization of nucleolar genes. Science (New York, N.Y.), 164(3882), 955-957.

Mitchell, P., Petfalski, E., \& Tollervey, D. (1996). The 3' end of yeast 5.8S rRNA is generated by an exonuclease processing mechanism. Genes \& Development, 10(4), 502-513.

Mohr, G., Del Campo, M., Mohr, S., Yang, Q., Jia, H., Jankowsky, E., \& Lambowitz, A. M. (2008). Function of the C-terminal Domain of the DEAD-box Protein Mss116p Analyzed in Vivo and in Vitro. Journal of Molecular Biology, 375(5), 1344-1364.

Mohr, G., Del Campo, M., Turner, K. G., Gilman, B., Wolf, R. Z., \& Lambowitz, A. M. (2011). High-Throughput Genetic Identification of Functionally Important Regions of the Yeast DEAD-Box Protein Mss116p. Journal of Molecular Biology, 413(5), 952-972.

Mullineux, S.-T., \& Lafontaine, D. L. J. (2012). Mapping the cleavage sites on mammalian pre-rRNAs: Where do we stand? Biochimie, 94(7), 1521-1532.

Murakami, K., Nakano, K., Shimizu, T., \& Ohto, U. (2017). The crystal structure of human DEAH-box RNA helicase 15 reveals a domain organization of the mammalian DEAH/RHA family. Acta Crystallographica. Section F, Structural Biology Communications, 73(Pt 6), 347.

Neumann, B., Wu, H., Hackmann, A., \& Krebber, H. (2016). Nuclear Export of PreRibosomal Subunits Requires Dbp5, but Not as an RNA-Helicase as for mRNA Export. PLOS ONE, 11(2).

Ni, J., Tien, A. L., \& Fournier, M. J. (1997). Small nucleolar RNAs direct site-specific synthesis of pseudouridine in ribosomal RNA. Cell, 89(4), 565-573.

Noble, C. G., \& Song, H. (2007). MLN51 Stimulates the RNA-Helicase Activity of elF4AllI. PLOS ONE, 2(3), e303.

Oeffinger, M., \& Tollervey, D. (2003). Yeast Nop15p is an RNA-binding protein required for pre-rRNA processing and cytokinesis. The EMBO Journal, 22(24), 6573-6583.

Oeffinger, M., Zenklusen, D., Ferguson, A., Wei, K. E., El Hage, A., Tollervey, D., Chait, B. T., Singer, R. H., \& Rout, M. P. (2009). Rrp17p is a eukaryotic exonuclease required for 5' end processing of Pre-60S ribosomal RNA. Molecular Cell, 36(5), 768-781.

Ojha, S., Malla, S., \& Lyons, S. M. (2020). snoRNPs: Functions in Ribosome Biogenesis. Biomolecules, 10(5), 783.

Osheim, Y. N., French, S. L., Keck, K. M., Champion, E. A., Spasov, K., Dragon, F., Baserga, S. J., \& Beyer, A. L. (2004). Pre-18S ribosomal RNA is structurally compacted into the SSU processome prior to being cleaved from nascent transcripts in Saccharomyces cerevisiae. Molecular Cell, 16(6), 943-954.

Owttrim, G. W. (2013). RNA helicases. RNA Biology, 10(1), 96-110. 
Ozgur, S., Buchwald, G., Falk, S., Chakrabarti, S., Prabu, J. R., \& Conti, E. (2015). The conformational plasticity of eukaryotic RNA-dependent ATPases. The FEBS Journal, 282(5), 850-863.

Parfentev, I., Schilbach, S., Cramer, P., \& Urlaub, H. (2020). An experimentally generated peptide database increases the sensitivity of XL-MS with complex samples. Journal of Proteomics, 220, 103754.

Pause, A., Méthot, N., \& Sonenberg, N. (1993). The HRIGRXXR region of the DEAD box RNA helicase eukaryotic translation initiation factor $4 A$ is required for RNA binding and ATP hydrolysis. Molecular and Cellular Biology, 13(11), 6789-6798.

Pause, A., \& Sonenberg, N. (1992). Mutational analysis of a DEAD box RNA helicase: The mammalian translation initiation factor elF-4A. The EMBO Journal, 11(7), 2643-2654.

Pertschy, B., Schneider, C., Gnädig, M., Schäfer, T., Tollervey, D., \& Hurt, E. (2009). RNA helicase Prp43 and its co-factor Pfa1 promote 20 to $18 \mathrm{~S}$ rRNA processing catalyzed by the endonuclease Nob1. The Journal of Biological Chemistry, 284(50), 3507935091.

Petrov, A. S., Bernier, C. R., Gulen, B., Waterbury, C. C., Hershkovits, E., Hsiao, C., Harvey, S. C., Hud, N. V., Fox, G. E., Wartell, R. M., \& Williams, L. D. (2014a). Secondary Structures of rRNAs from All Three Domains of Life. PLOS ONE, 9(2), e88222.

Petrov, A. S., Bernier, C. R., Hsiao, C., Norris, A. M., Kovacs, N. A., Waterbury, C. C., Stepanov, V. G., Harvey, S. C., Fox, G. E., Wartell, R. M., Hud, N. V., \& Williams, L. D. (2014b). Evolution of the ribosome at atomic resolution. Proceedings of the National Academy of Sciences of the United States of America, 111(28), 1025110256.

Polikanov, Y. S., Melnikov, S. V., Söll, D., \& Steitz, T. A. (2015). Structural insights into the role of rRNA modifications in protein synthesis and ribosome assembly. Nature Structural \& Molecular Biology, 22(4), 342-344.

Ponting, C. P. (2000). Novel elF4G domain homologues linking mRNA translation with nonsense-mediated mRNA decay. Trends in Biochemical Sciences, 25(9), 423-426.

Pyle, A. M. (2008). Translocation and Unwinding Mechanisms of RNA and DNA Helicases. Annual Review of Biophysics, 37(1), 317-336.

Qamar, S., Kramer, K., \& Urlaub, H. (2015). Chapter Fourteen-Studying RNA-Protein Interactions of Pre-mRNA Complexes by Mass Spectrometry. In S. A. Woodson \& F. H. T. Allain (Eds.), Methods in Enzymology (Vol. 558, pp. 417-463). Academic Press.

Qu, G., van Nues, R. W., Watkins, N. J., \& Maxwell, E. S. (2011). The spatial-functional coupling of box C/D and C'/D' RNPs is an evolutionarily conserved feature of the eukaryotic box C/D snoRNP nucleotide modification complex. Molecular and Cellular Biology, 31(2), 365-374. 
Raj, S., Bagchi, D., Orero, J. V., Banroques, J., Tanner, N. K., \& Croquette, V. (2019). Mechanistic characterization of the DEAD-box RNA helicase Ded1 from yeast as revealed by a novel technique using single-molecule magnetic tweezers. Nucleic Acids Research, 47(7), 3699-3710.

Ranji, A., \& Boris-Lawrie, K. (2010). RNA helicases. RNA Biology, 7(6), 775-787.

Rawling, D. C., \& Pyle, A. M. (2014). Parts, assembly and operation of the RIG-I family of motors. Current Opinion in Structural Biology, 0, 25-33.

Reichow, S. L., Hamma, T., Ferré-D’Amaré, A. R., \& Varani, G. (2007). The structure and function of small nucleolar ribonucleoproteins. Nucleic Acids Research, 35(5), 14521464.

Rogers, G. W., Lima, W. F., \& Merrick, W. C. (2001). Further characterization of the helicase activity of elF4A. Substrate specificity. The Journal of Biological Chemistry, 276(16), 12598-12608.

Rogers, G. W., Richter, N. J., \& Merrick, W. C. (1999). Biochemical and kinetic characterization of the RNA helicase activity of eukaryotic initiation factor $4 \mathrm{~A}$. The Journal of Biological Chemistry, 274(18), 12236-12244.

Rosado, I. V., \& Cruz, J. D. L. (2004). Npa1p is an essential trans-acting factor required for an early step in the assembly of $60 \mathrm{~S}$ ribosomal subunits in Saccharomyces cerevisiae. RNA, 10(7), 1073-1083.

Rosado, I. V., Dez, C., Lebaron, S., Caizergues-Ferrer, M., Henry, Y., \& de la Cruz, J. (2007a). Characterization of Saccharomyces cerevisiae Npa2p (Urb2p) Reveals a Low-Molecular-Mass Complex Containing Dbp6p, Npa1p (Urb1p), Nop8p, and Rsa3p Involved in Early Steps of 60S Ribosomal Subunit Biogenesis. Molecular and Cellular Biology, 27(4), 1207-1221.

Rosado, I. V., Kressler, D., \& de la Cruz, J. (2007b). Functional analysis of Saccharomyces cerevisiae ribosomal protein Rpl3p in ribosome synthesis. Nucleic Acids Research, 35(12), 4203-4213.

Rudolph, M. G., \& Klostermeier, D. (2009). The Thermus thermophilus DEAD box helicase Hera contains a modified RNA recognition motif domain loosely connected to the helicase core. RNA, 15(11), 1993-2001.

Rudolph, M. G., \& Klostermeier, D. (2015). When core competence is not enough: Functional interplay of the DEAD-box helicase core with ancillary domains and auxiliary factors in RNA binding and unwinding. Biological Chemistry, 396(8), 849-865.

Sahasranaman, A., Dembowski, J., Strahler, J., Andrews, P., Maddock, J., \& Woolford, J. L. (2011). Assembly of Saccharomyces cerevisiae 60S ribosomal subunits: Role of factors required for $27 \mathrm{~S}$ pre-rRNA processing. The EMBO Journal, 30(19), 40204032. 
Sanghai, Z. A., Miller, L., Molloy, K. R., Barandun, J., Hunziker, M., Chaker-Margot, M., Wang, J., Chait, B. T., \& Klinge, S. (2018). Modular assembly of the nucleolar pre$60 S$ ribosomal subunit. Nature, 556(7699), 126-129.

Sardana, R., Liu, X., Granneman, S., Zhu, J., Gill, M., Papoulas, O., Marcotte, E. M., Tollervey, D., Correll, C. C., \& Johnson, A. W. (2015). The DEAH-box helicase Dhr1 dissociates U3 from the pre-rRNA to promote formation of the central pseudoknot. PLoS Biology, 13(2), e1002083.

Schmitt, M. E., \& Clayton, D. A. (1993). Nuclear RNase MRP is required for correct processing of pre-5.8S rRNA in Saccharomyces cerevisiae. Molecular and Cellular Biology, 13(12), 7935-7941.

Schuller, J. M., Falk, S., Fromm, L., Hurt, E., \& Conti, E. (2018). Structure of the nuclear exosome captured on a maturing preribosome. Science, 360(6385), 219-222.

Schur, F. K. (2019). Toward high-resolution in situ structural biology with cryo-electron tomography and subtomogram averaging. Current Opinion in Structural Biology, 58, $1-9$.

Schütz, P., Bumann, M., Oberholzer, A. E., Bieniossek, C., Trachsel, H., Altmann, M., \& Baumann, U. (2008). Crystal structure of the yeast elF4A-elF4G complex: An RNAhelicase controlled by protein-protein interactions. Proceedings of the National Academy of Sciences, 105(28), 9564-9569.

Semlow, D. R., Blanco, M. R., Walter, N. G., \& Staley, J. P. (2016). Spliceosomal DEAH-Box ATPases Remodel Pre-mRNA to Activate Alternative Splice Sites. Cell, 164(5), 985998.

Sengoku, T., Nureki, O., Nakamura, A., Kobayashi, S., \& Yokoyama, S. (2006). Structural Basis for RNA Unwinding by the DEAD-Box Protein Drosophila Vasa. Cell, 125(2), 287-300.

Sharif, H., Ozgur, S., Sharma, K., Basquin, C., Urlaub, H., \& Conti, E. (2013). Structural analysis of the yeast Dhh1-Pat1 complex reveals how Dhh1 engages Pat1, Edc3 and RNA in mutually exclusive interactions. Nucleic Acids Research, 41(17), 8377-8390.

Sharma, S., \& Lafontaine, D. L. J. (2015). "View From A Bridge": A New Perspective on Eukaryotic rRNA Base Modification. Trends in Biochemical Sciences, 40(10), 560575.

Sharma, S., Langhendries, J.-L., Watzinger, P., Kötter, P., Entian, K.-D., \& Lafontaine, D. L. J. (2015). Yeast Kre33 and human NAT10 are conserved 18S rRNA cytosine acetyltransferases that modify tRNAs assisted by the adaptor Tan1/THUMPD1. Nucleic Acids Research, 43(4), 2242-2258.

Sharma, S., Yang, J., Nues, R. van, Watzinger, P., Kötter, P., Lafontaine, D. L. J., Granneman, S., \& Entian, K.-D. (2017). Specialized box C/D snoRNPs act as antisense guides to target RNA base acetylation. PLOS Genetics, 13(5), e1006804. 
Shimoji, K., Jakovljevic, J., Tsuchihashi, K., Umeki, Y., Wan, K., Kawasaki, S., Talkish, J., Woolford, J. L., \& Mizuta, K. (2012). Ebp2 and Brx1 function cooperatively in 60S ribosomal subunit assembly in Saccharomyces cerevisiae. Nucleic Acids Research, 40(10), 4574-4588.

Shuai, K., \& Warner, J. R. (1991). A temperature sensitive mutant of Saccharomyces cerevisiae defective in pre-rRNA processing. Nucleic Acids Research, 19(18), 50595064.

Singleton, M. R., Dillingham, M. S., \& Wigley, D. B. (2007). Structure and Mechanism of Helicases and Nucleic Acid Translocases. Annual Review of Biochemistry, 76(1), 2350.

Sloan, K. E., \& Bohnsack, M. T. (2018). Unravelling the Mechanisms of RNA Helicase Regulation. Trends in Biochemical Sciences, 43(4), 237-250.

Sloan, K. E., Gleizes, P.-E., \& Bohnsack, M. T. (2016). Nucleocytoplasmic Transport of RNAs and RNA-Protein Complexes. Journal of Molecular Biology, 428(10, Part A), 2040-2059.

Sloan, K. E., Leisegang, M. S., Doebele, C., Ramírez, A. S., Simm, S., Safferthal, C., Kretschmer, J., Schorge, T., Markoutsa, S., Haag, S., Karas, M., Ebersberger, I., Schleiff, E., Watkins, N. J., \& Bohnsack, M. T. (2015). The association of late-acting snoRNPs with human pre-ribosomal complexes requires the RNA helicase DDX21. Nucleic Acids Research, 43(1), 553-564.

Sloan, K. E., Warda, A. S., Sharma, S., Entian, K.-D., Lafontaine, D. L. J., \& Bohnsack, M. T. (2017). Tuning the ribosome: The influence of rRNA modification on eukaryotic ribosome biogenesis and function. RNA Biology, 14(9), 1138-1152.

Soltanieh, S., Osheim, Y. N., Spasov, K., Trahan, C., Beyer, A. L., \& Dragon, F. (2015). DEAD-Box RNA Helicase Dbp4 Is Required for Small-Subunit Processome Formation and Function. Molecular and Cellular Biology, 35(5), 816-830.

Song, C., Hotz-Wagenblatt, A., Voit, R., \& Grummt, I. (2017). SIRT7 and the DEAD-box helicase DDX21 cooperate to resolve genomic $R$ loops and safeguard genome stability. Genes \& Development, 31(13), 1370-1381.

Steckelberg, A.-L., Boehm, V., Gromadzka, A. M., \& Gehring, N. H. (2012). CWC22 Connects Pre-mRNA Splicing and Exon Junction Complex Assembly. Cell Reports, 2(3), 454-461.

Strunk, B. S., \& Karbstein, K. (2009). Powering through ribosome assembly. RNA (New York, N.Y.), 15(12), 2083-2104.

Studer, M. K., Ivanović, L., Weber, M. E., Marti, S., \& Jonas, S. (2020). Structural basis for DEAH-helicase activation by G-patch proteins. Proceedings of the National Academy of Sciences, 117(13), 7159-7170. 
Stützer, A., Chernev, A., \& Urlaub, H. (2016). Protein-Nukleinsäure-Interaktionen im Massenspektrometer. BIOspektrum, 22(5), 466-469.

Sun, C., \& Woolford, J. L. (1997). The yeast nucleolar protein Nop4p contains four RNA recognition motifs necessary for ribosome biogenesis. The Journal of Biological Chemistry, 272(40), 25345-25352.

Svitkin, Y. V., Pause, A., Haghighat, A., Pyronnet, S., Witherell, G., Belsham, G. J., \& Sonenberg, N. (2001). The requirement for eukaryotic initiation factor 4A (elF4A) in translation is in direct proportion to the degree of mRNA 5' secondary structure. RNA, 7(3), 382-394.

Swaney, D. L., Beltrao, P., Starita, L., Guo, A., Rush, J., Fields, S., Krogan, N. J., \& Villén, J. (2013). Global analysis of phosphorylation and ubiquitylation crosstalk in protein degradation. Nature Methods, 10(7).

Szewczak, L. B. W., DeGregorio, S. J., Strobel, S. A., \& Steitz, J. A. (2002). Exclusive interaction of the $15.5 \mathrm{kD}$ protein with the terminal box $\mathrm{C} / \mathrm{D}$ motif of a methylation guide snoRNP. Chemistry \& Biology, 9(10), 1095-1107.

Talkish, J., Campbell, I. W., Sahasranaman, A., Jakovljevic, J., \& Woolford, J. L. (2014). Ribosome assembly factors Pwp1 and Nop12 are important for folding of 5.8S rRNA during ribosome biogenesis in Saccharomyces cerevisiae. Molecular and Cellular Biology, 34(10), 1863-1877.

Talwar, T., Vidhyasagar, V., Qing, J., Guo, M., Kariem, A., Lu, Y., Singh, R. S., Lukong, K. E., \& Wu, Y. (2017). The DEAD-box protein DDX43 (HAGE) is a dual RNA-DNA helicase and has a K-homology domain required for full nucleic acid unwinding activity. Journal of Biological Chemistry, 292(25), 10429-10443.

Tanaka, N., Aronova, A., \& Schwer, B. (2007). Ntr1 activates the Prp43 helicase to trigger release of lariat-intron from the spliceosome. Genes \& Development, 21(18), 23122325.

Tanaka, N., \& Schwer, B. (2005). Characterization of the NTPase, RNA-Binding, and RNA Helicase Activities of the DEAH-Box Splicing Factor Prp22. Biochemistry, 44(28), 9795-9803.

Tang, L., Sahasranaman, A., Jakovljevic, J., Schleifman, E., \& Woolford, J. L. (2008). Interactions among Ytm1, Erb1, and Nop7 required for assembly of the Nop7subcomplex in yeast preribosomes. Molecular Biology of the Cell, 19(7), 2844-2856.

Tanner, N. K., Cordin, O., Banroques, J., Doère, M., \& Linder, P. (2003). The Q Motif: A Newly Identified Motif in DEAD Box Helicases May Regulate ATP Binding and Hydrolysis. Molecular Cell, 11(1), 127-138. https://doi.org/10.1016/S10972765(03)00006-6

Tanner, N. K., \& Linder, P. (2001). DExD/H Box RNA Helicases: From Generic Motors to Specific Dissociation Functions. Molecular Cell, 8(2), 251-262. 
Taoka, M., Nobe, Y., Yamaki, Y., Yamauchi, Y., Ishikawa, H., Takahashi, N., Nakayama, H., \& Isobe, T. (2016). The complete chemical structure of Saccharomyces cerevisiae rRNA: Partial pseudouridylation of U2345 in $25 \mathrm{~S}$ rRNA by snoRNA snR9. Nucleic Acids Research, 44(18), 8951-8961.

Tauchert, M. J., Fourmann, J.-B., Lührmann, R., \& Ficner, R. (2017). Structural insights into the mechanism of the DEAH-box RNA helicase Prp43. ELife, 6, e21510.

Theissen, B., Karow, A. R., Köhler, J., Gubaev, A., \& Klostermeier, D. (2008). Cooperative binding of ATP and RNA induces a closed conformation in a DEAD box RNA helicase. Proceedings of the National Academy of Sciences of the United States of America, 105(2), 548-553.

Thoms, M., Thomson, E., Baßler, J., Gnädig, M., Griesel, S., \& Hurt, E. (2015). The Exosome Is Recruited to RNA Substrates through Specific Adaptor Proteins. Cell, 162(5), 1029-1038.

Tollervey, D., Lehtonen, H., Jansen, R., Kern, H., \& Hurt, E. C. (1993). Temperaturesensitive mutations demonstrate roles for yeast fibrillarin in pre-rRNA processing, prerRNA methylation, and ribosome assembly. Cell, 72(3), 443-457.

Tran, E. J., Zhou, Y., Corbett, A. H., \& Wente, S. R. (2007). The DEAD-Box Protein Dbp5 Controls mRNA Export by Triggering Specific RNA:Protein Remodeling Events. Molecular Cell, 28(5), 850-859.

Trapman, J., Retèl, J., \& Planta, R. J. (1975). Ribosomal precursor particles from yeast. Experimental Cell Research, 90(1), 95-104.

Tsu, C. A., Kossen, K., \& Uhlenbeck, O. C. (2001). The Escherichia coli DEAD protein DbpA recognizes a small RNA hairpin in 23S rRNA. RNA, 7(5), 702-709.

Turowski, T. W., \& Tollervey, D. (2015). Cotranscriptional events in eukaryotic ribosome synthesis. Wiley Interdisciplinary Reviews. RNA, 6(1), 129-139.

Udem, S. A., \& Warner, J. R. (1972). Ribosomal RNA synthesis in Saccharomyces cerevisiae. Journal of Molecular Biology, 65(2), 227-242.

Uhlmann-Schiffler, H., Jalal, C., \& Stahl, H. (2006). Ddx42p-A human DEAD box protein with RNA chaperone activities. Nucleic Acids Research, 34(1), 10-22.

van Nues, R. W., Granneman, S., Kudla, G., Sloan, K. E., Chicken, M., Tollervey, D., \& Watkins, N. J. (2011). Box C/D snoRNP catalysed methylation is aided by additional pre-rRNA base-pairing. The EMBO Journal, 30(12), 2420-2430.

van Nues, R. W., \& Watkins, N. J. (2017). Unusual C'/D' motifs enable box C/D snoRNPs to modify multiple sites in the same rRNA target region. Nucleic Acids Research, 45(4), 2016-2028. 
Venema, J., \& Tollervey, D. (1995). Processing of pre-ribosomal RNA in Saccharomyces cerevisiae. Yeast (Chichester, England), 11(16), 1629-1650.

Wang, S., Hu, Y., Overgaard, M. T., Karginov, F. V., Uhlenbeck, O. C., \& McKay, D. B. (2006). The domain of the Bacillus subtilis DEAD-box helicase YxiN that is responsible for specific binding of $23 \mathrm{~S}$ rRNA has an RNA recognition motif fold. RNA, 12(6), 959-967.

Warner, J. R. (1999). The economics of ribosome biosynthesis in yeast. Trends in Biochemical Sciences, 24(11), 437-440.

Watkins, N. J., Ségault, V., Charpentier, B., Nottrott, S., Fabrizio, P., Bachi, A., Wilm, M., Rosbash, M., Branlant, C., \& Lührmann, R. (2000). A common core RNP structure shared between the small nucleoar box C/D RNPs and the spliceosomal U4 snRNP. Cell, 103(3), 457-466.

Watkins, Nicholas J., \& Bohnsack, M. T. (2012). The box C/D and H/ACA snoRNPs: Key players in the modification, processing and the dynamic folding of ribosomal RNA. Wiley Interdisciplinary Reviews. RNA, 3(3), 397-414.

Weaver, P. L., Sun, C., \& Chang, T. H. (1997). Dbp3p, a putative RNA helicase in Saccharomyces cerevisiae, is required for efficient pre-rRNA processing predominantly at site A3. Molecular and Cellular Biology, 17(3), 1354-1365.

Weirich, C. S., Erzberger, J. P., Flick, J. S., Berger, J. M., Thorner, J., \& Weis, K. (2006). Activation of the $\mathrm{DExD} / \mathrm{H}$-box protein $\mathrm{Dbp} 5$ by the nuclear-pore protein $\mathrm{Gle} 1$ and its coactivator InsP 6 is required for mRNA export. Nature Cell Biology, 8(7), 668-676.

Wells, G. R., Weichmann, F., Colvin, D., Sloan, K. E., Kudla, G., Tollervey, D., Watkins, N. J., \& Schneider, C. (2016). The PIN domain endonuclease Utp24 cleaves preribosomal RNA at two coupled sites in yeast and humans. Nucleic Acids Research, 44(11), 5399-5409.

White, J., Li, Z., Sardana, R., Bujnicki, J. M., Marcotte, E. M., \& Johnson, A. W. (2008). Bud23 Methylates G1575 of 18S rRNA and Is Required for Efficient Nuclear Export of Pre-40S Subunits. Molecular and Cellular Biology, 28(10), 3151-3161.

Wilson, D. N., \& Doudna Cate, J. H. (2012). The structure and function of the eukaryotic ribosome. Cold Spring Harbor Perspectives in Biology, 4(5).

Woolford, J. L., \& Baserga, S. J. (2013). Ribosome Biogenesis in the Yeast Saccharomyces cerevisiae. Genetics, 195(3), 643-681.

Wu, S., Tutuncuoglu, B., Yan, K., Brown, H., Zhang, Y., Tan, D., Gamalinda, M., Yuan, Y., Li, Z., Jakovljevic, J., Ma, C., Lei, J., Dong, M.-Q., Woolford, J. L., \& Gao, N. (2016). Diverse roles of assembly factors revealed by structures of late nuclear pre-60S ribosomes. Nature, 534(7605), 133-137. 
Yang, Q., Campo, M. D., Lambowitz, A. M., \& Jankowsky, E. (2007). DEAD-Box Proteins Unwind Duplexes by Local Strand Separation. Molecular Cell, 28(2), 253-263.

Yang, Q., \& Jankowsky, E. (2006). The DEAD-box protein Ded1 unwinds RNA duplexes by a mode distinct from translocating helicases. Nature Structural \& Molecular Biology, 13(11), 981-986.

Yoneyama, M., \& Fujita, T. (2008). Structural mechanism of RNA recognition by the RIG-Ilike receptors. Immunity, 29(2), 178-181.

Yu, C., \& Huang, L. (2018). Cross-Linking Mass Spectrometry (XL-MS): An Emerging Technology for Interactomics and Structural Biology. Analytical Chemistry, 90(1), 144-165.

Zhang, P. (2019). Advances in cryo-electron tomography and subtomogram averaging and classification. Current Opinion in Structural Biology, 58, 249-258.

Zhou, D., Zhu, X., Zheng, S., Tan, D., Dong, M.-Q., \& Ye, K. (2019). Cryo-EM structure of an early precursor of large ribosomal subunit reveals a half-assembled intermediate. Protein \& Cell, 10(2), 120-130.

Zhou, Y., Musalgaonkar, S., Johnson, A. W., \& Taylor, D. W. (2019). Tightly-orchestrated rearrangements govern catalytic center assembly of the ribosome. Nature Communications, 10.

Zorbas, C., Nicolas, E., Wacheul, L., Huvelle, E., Heurgué-Hamard, V., \& Lafontaine, D. L. J. (2015). The human 18S rRNA base methyltransferases DIMT1L and WBSCR22TRMT112 but not rRNA modification are required for ribosome biogenesis. Molecular Biology of the Cell, 26(11), 2080-209 


\section{Acknowledgement}

First of all, I would like to thank the International Max Planck Research School and the Max Planck Society for their initiatives to grant opportunities to young aspiring scientists to come to Germany and acquire higher training. You provided me with the boat to leave the shore of my country with hopes of doing better science. I am definitely transformed with so much growth and knowledge to partake in the training of the next generation of scientists of my country.

To my supervisors, Markus and Katherine Bohnsack. Thank you for letting me enter Room 0.226 to sharpen my "pipetting" skills, and for helping me develop and enhance my scientific thinking and writing. I will be forever grateful for every career milestone I will have in the future resulting from your dedicated supervision. More than being my advisors, a big thank you for being great mentors.

To my previous mentors, my advanced biology and research teachers in high school, Ma'am Larina Taborda and Lourdes Medrano respectively, thank you for getting me acquainted with the beauty of the central dogma of molecular biology and for igniting my passion for research. To the faculty of the National Institute of Molecular Biology and

Biotechnology of the University of the Philippines-Diliman, thank you for providing me the early and fundamental training to have the proper armor in exploring things at the molecular level. Special thanks to my former principal investigator, Dr. Jose Enrico Lazaro.

To my parents (mama Mercy and papa Gerry), who do not have the slightest idea of what exactly I am working on but never fails to support and encourage me since I started chasing those childhood dreams, thank you for always giving me the freedom to be who I am and to pursue what I want to be in life. Your massive love has been my power in conquering the world and my aspirations.

To my lovely brothers (Jerick and Jan Michael), my constant cheerleaders, I am thankful for our constant drive to inspire each other to reach our dreams. You guys have been my source of encouragement and my inflatable airbag or trampoline when failure strikes.

To my second mom (mama Emily), you are the one who inspired me to become a scientist. Thank you for all of your love and support, and for being the first one to wish me the best and to say a prayer in every endeavor that I face.

To my late grandparents, Mariano and Martina Rabara, who were always there whenever I received a medal or a ribbon of recognition, I dedicate this degree to both of you. I always feel your love and guidance from above. I am also grateful to the Aquino and Rabara clan, here is another Dr. to the family.

To the 'sehr nette' Filipino people I have met outside our islands - Joy, Julius, and Sarah: thank you for being with me during those days I was struggling to become whole again; for our cooking sessions, watch parties, and spontaneous meetings; and for being the best people to surround myself in both good and bad days.

To the beautiful people (Sung-Hui, Yen-Yun, and Yi-Tse) from the east side of the world who also ventured to do their graduate studies overseas, thank you for all the support in my $\mathrm{PhD}$ and personal life. You made me feel I am not alone in this journey and so this also goes out for you guys.

To Roemel and Samuel, thank you for the segments of our lives we shared to experience genuine love and appreciation, and to learn about ourselves even more. Thank you for all the 
wonderful things that happened between our first "hello" and the last "goodbye". You gave flavor to my PhD journey.

To my Ates (Ate Lisa, Janette, Jen, Clarissa), thank you for voluntarily acting as my big sisters and second moms in Göttingen. Thank you for all the good and comforting food, and for throwing birthday parties for me filled with dancing, singing, and laughter. I am also grateful to the Filipino community in Göttingen for the "kababayan" spirit and for bringing a semblance of home to Göttingen.

To the people in the lab and in the department, thank you for giving the rich experience to work with diverse people and to learn camaraderie. Special thanks to Priyanka, Nidhi, Philipp, and Rebecca for being more than just colleagues. I am also grateful to our lab extensions, our collaborators in Göttingen, Copenhagen and New Castle, for sharing their expertise to broaden the scope of this work. Special thanks to Prof. Dr. Henning Urlaub, Dr. Kuan-Ting Pan, Dr. Nicolai Krogh and Dr. Henrik Nielsen.

To Steffen Burkhardt and Kerstin Grüniger, thank you for undertaking all the logistics concerning getting my foreign pass, boarding my plane, getting out of the train station, and reaching my first home outside home; and for dealing all the German bureaucracies that surely are overwhelming for an Ausländer. You are definitely one of the reasons behind the good name and reputation of the IMPRS-MolBio program.

To my volleyball team, especially to our coach and the friends I made, thank you for letting me hit the ball hard after a frustrating day in the lab. It was a nice source of progress especially on the days when I am stuck in my PhD project.

To the RNA helicases, especially Dbp7 and Dbp3, for teaching me how to "unwind" and when to use NTP efficiently and effectively.

And to the many connections I have made in the various corners of Göttingen-from Döner shops, DHL deliveries, German classes, Wilhelmplatz, salsa courses, beach volleyball courts, i-survived-the-fire-party group, Asian stores, flatmates, students of the program, collaborations, conferences, random encounters, and many more-many thanks as well! You all provided me a window to see the world with your stories, and you all made this journey colorful and holistic.

To all the smiles, the tears, the doubts, the struggles, the bad and good memories, the feeling of being lost, the fleeting moments, the best and worst days, the heartbreaks, and the internal battles that came along this journey- you all shaped me to be stronger and to keep on keeping on.

Thank you to the universe for not letting me miss what is meant for me and to all the stars that aligned that got me to this point.

To myself, for dreaming, believing, administering care and compassion when breaking point is close, you deserve a pat on the back for making it this far.

Vielen Dank.

Maraming salamat.

Agyaman ak. 